\title{
Cholesterol efflux as a measure of HDL functionality in humans
}

Citation for published version (APA):

Talbot, C. P. J. (2018). Cholesterol efflux as a measure of HDL functionality in humans: impact of genetics, diet and weight loss. [Doctoral Thesis, Maastricht University]. Gildeprint Drukkerijen. https://doi.org/10.26481/dis.20180419ct

Document status and date:

Published: 01/01/2018

DOI:

$10.26481 /$ dis.20180419ct

Document Version:

Publisher's PDF, also known as Version of record

\section{Please check the document version of this publication:}

- A submitted manuscript is the version of the article upon submission and before peer-review. There can be important differences between the submitted version and the official published version of record.

People interested in the research are advised to contact the author for the final version of the publication, or visit the DOI to the publisher's website.

- The final author version and the galley proof are versions of the publication after peer review.

- The final published version features the final layout of the paper including the volume, issue and page numbers.

Link to publication

\footnotetext{
General rights rights.

- You may freely distribute the URL identifying the publication in the public portal. please follow below link for the End User Agreement:

www.umlib.nl/taverne-license

Take down policy

If you believe that this document breaches copyright please contact us at:

repository@maastrichtuniversity.nl

providing details and we will investigate your claim.
}

Copyright and moral rights for the publications made accessible in the public portal are retained by the authors and/or other copyright owners and it is a condition of accessing publications that users recognise and abide by the legal requirements associated with these

- Users may download and print one copy of any publication from the public portal for the purpose of private study or research.

- You may not further distribute the material or use it for any profit-making activity or commercial gain

If the publication is distributed under the terms of Article $25 \mathrm{fa}$ of the Dutch Copyright Act, indicated by the "Taverne" license above, 
Cholesterol efflux as a measure of HDL functionality in humans: impact of genetics, diet and weight loss 


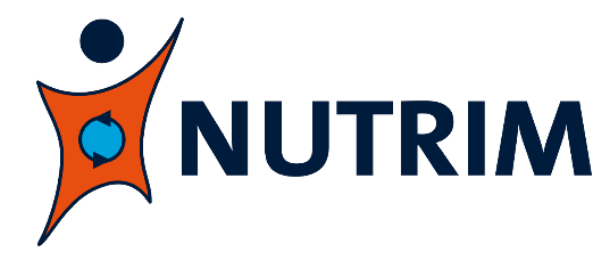

The studies presented in this thesis were performed within NUTRIM School of Nutrition and Translational Research in Metabolism.

Cover design: Evelien Jagtman - evelienjagtman.com

Layout: Charlotte Talbot

Printed by: Gildeprint

(c) Charlotte Talbot, Maastricht 2018

ISBN: 978-94-6233-899-9 


\section{Cholesterol efflux as a measure of HDL functionality in humans: impact of genetics, diet and weight loss}

\section{DISSERTATION}

To obtain the degree of doctor at the Maastricht University, on the authority of the Rector Magnificus,

Prof. dr. Rianne M. Letschert,

in accordance with the decision of the Board of Deans, to be defended in public on Thursday 19th April 2018 at 12:00 hours by Charlotte Pauline Joëlle Talbot born in Parthenay, France, on September 19th 1989 


\section{Supervisors}

Prof. dr. ir. R. P. Mensink

Prof. dr. J. Plat

\section{Assessment Committee}

Prof. dr. E.C.M. Mariman (chairman)

Prof. Dr. E.A.L. Biessen

Dr. C. van der Kallen

Prof. dr. B. Staels (Faculté de Pharmacie, Lille, France)

Dr. P.L Zock (Unilever R\&D, Vlaardingen, The Netherlands) 


\section{Table of Contents}

$\begin{array}{lll}\text { Chapter } 1 & \text { General introduction } & 7\end{array}$

Chapter 2 Determinants of cholesterol efflux capacity in humans 23

Chapter $3 \quad$ SNPs located in ABCA1, ABCG1, LXR $\beta$ and CETP are 57

associated with plasma cholesterol efflux

Chapter $4 \quad$ Theobromine does not affect fasting and postprandial HDL 77

cholesterol efflux capacity, while it decreases fasting miR-92a

levels in humans

Chapter 5 Effects of plant sterol and stanol ester enriched margarine

99

consumption on plasma cholesterol efflux capacity,

CETP mass and CE transfer

Chapter 6 HDL cholesterol efflux capacity and CETP activity are

associated with body mass, but are not changed by

diet-induced weight loss: a randomized trial in abdominally

obese men

Chapter $7 \quad$ General discussion

Summary

Résumé

Valorisation

Acknowledgements

Curriculum Vitae 


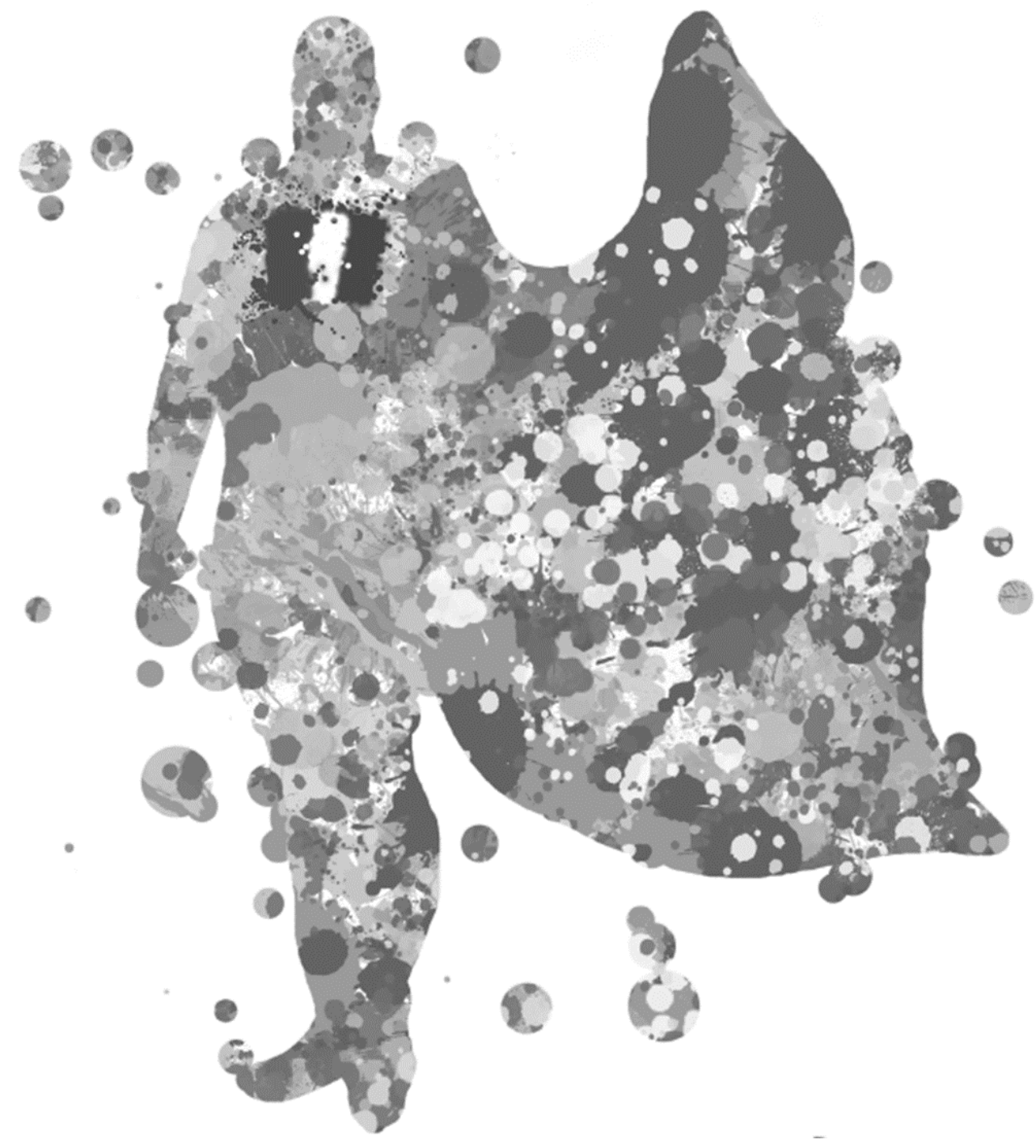




\section{Chapter 1}

\section{General introduction}




\section{Cardiovascular diseases}

Cardiovascular diseases (CVD) represent the leading cause of death worldwide and $31 \%$ of all global deaths are attributed to CVD [1]. CVD is defined by several diseases of the heart and blood vessels, and involve several disorders, including coronary heart disease (CHD), peripheral arterial disease and cerebrovascular disease. One of the underlying causes of CHD is atherosclerosis, which is actually a chronic inflammation of the arterial vascular wall, resulting in the recruitment of macrophages and lymphocytes and finally in the formation of a plaque. The plaque causes narrowing of the arterial lumen, obstructing partially or totally the blood flow to the heart or brain. An immediate cause of CHD is the rupture of such an atherosclerotic plaque (Figure 1) [2].

Normal artery

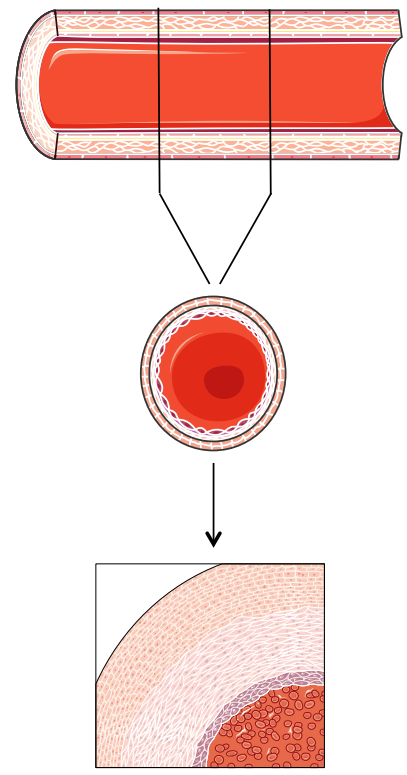

Artery narrowed by plaque

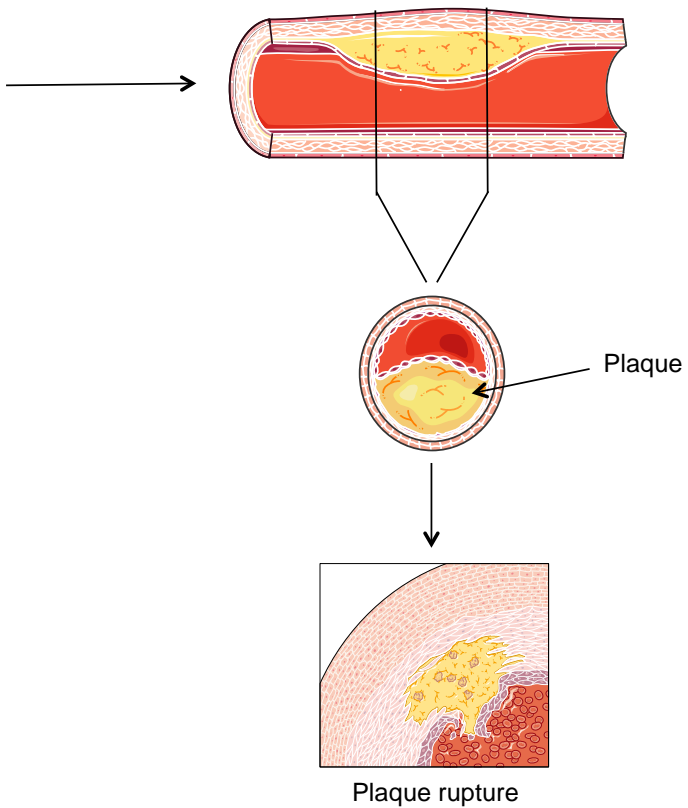

Figure 1: Development of atherosclerosis.

The first step in the development of atherosclerosis is the formation of fatty streaks, consisting of lipid-containing foam cells. These cells can then evolve into a "vulnerable" atherosclerotic plaque or remain stable. Eventually, the accumulation of a large amount of lipids and the formation of a thin fibrous cap can lead to the rupture of the atherosclerotic plaque, thereby causing thrombosis and myocardial infraction or stroke. The figure was produced using Servier Medical Art (http://smart.servier.com/). 
Many factors influence the risk to develop atherosclerosis, such as a lack of physical activity, cigarette smoking, an increased blood pressure, obesity, and type II diabetes. Moreover, an abnormal lipoprotein profile, also called dyslipidemia, characterized by a high level of low-density lipoprotein cholesterol (LDL-C) and a low level of high-density lipoprotein cholesterol (HDL-C), represents another important risk factor for atherosclerosis [3]. Lowering LDL-C concentrations is a proven target for interventions, and the most widely used drugs to lower serum LDL-C concentrations are statins [4,5]. By inhibiting the rate-limiting enzyme in cholesterol biosynthesis, HMG-CoA reductase, statins are highly effective in lowering the cholesterol production in the liver, leading to a reduction in LDL-C concentration due to an upregulation of the LDL-receptor (LDR-R) [6]. Although statins are associated with a reduced risk to develop CVD, many statin-treated patients fail to reach the desired LDL-C concentrations, which consequently lead to a lower protection against cardiovascular events [7]. Recently, a novel approach using monoclonal antibodies against PCSK9 (Proprotein convertase subtilisin/kexin type 9) has been developed. Inhibition of PCSK9 has been shown to increase the number of LDL-R on hepatocytes and to reduce LDL-C concentrations [8]. Recent data suggest that, when added to statin therapy, the inhibition of PCSK9 is associated with a further reduction in cardiovascular events on top of the effect of statins alone [9]. Moreover, although an inverse relationship between serum HDL$\mathrm{C}$ concentrations and CVD risk has been found in several epidemiological studies [10, 11], raising HDL-C levels failed to reduce CVD risk [12]. In this context, it appears essential to discover new strategies to efficiently reduce CVD risk via the HDL axis. An attractive strategy is increasing HDL functionality, rather than simply HDL-C concentrations [13].

\section{Lipoprotein metabolism}

Because cholesterol is insoluble in water, it has to be incorporated into lipoproteins in order to be transported throughout the body via the bloodstream. Lipoproteins are classified according their density. They are made of a central core containing cholesteryl esters (CE) and triacylglycerols (TAG), surrounded by free cholesterol, phospholipids, and apolipoproteins (Figure 2) [14]. 

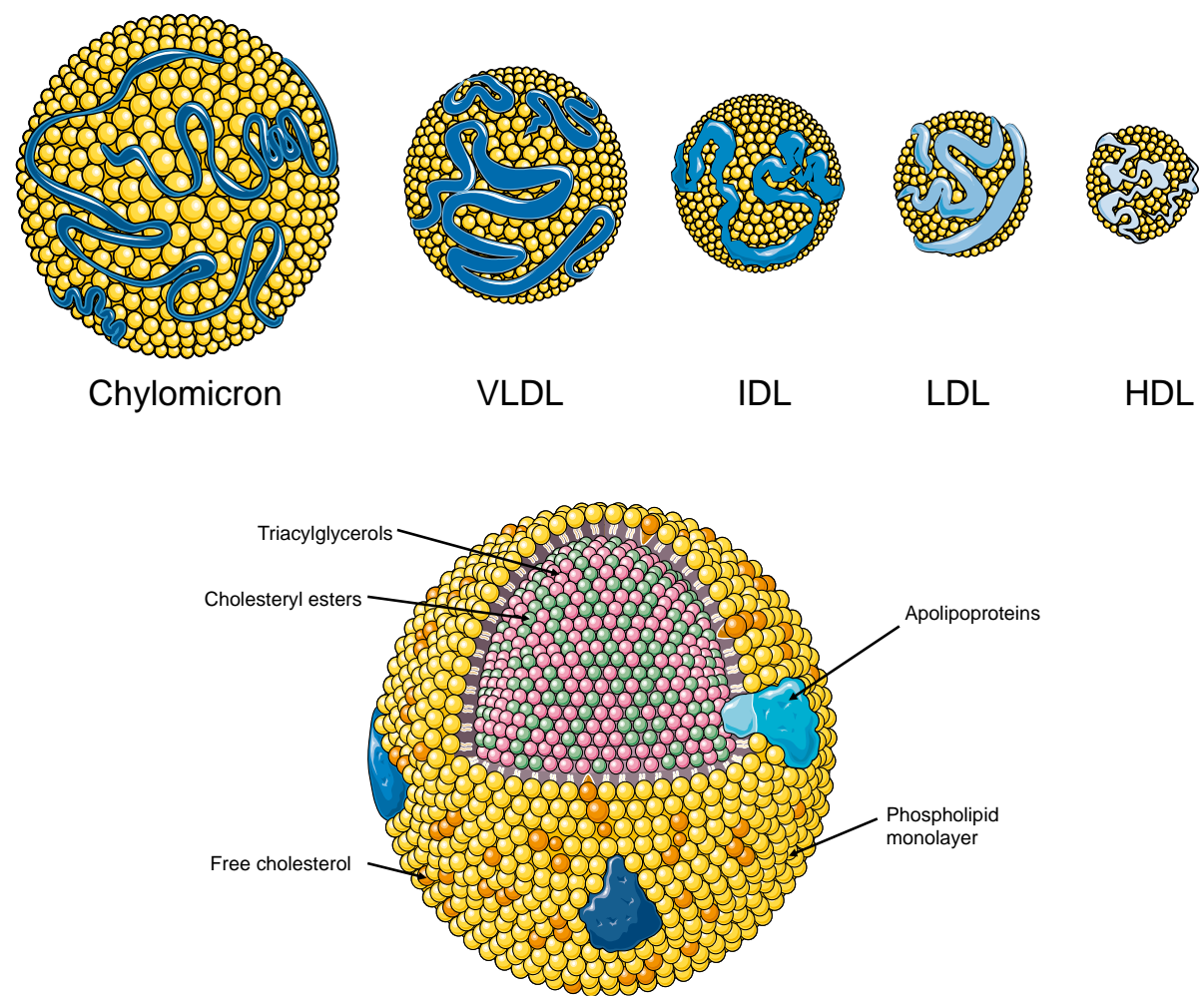

Figure 2: Lipoproteins classification and general composition.

VLDL: Very low-density lipoproteins; IDL: Intermediate-density lipoproteins; LDL: Low- density lipoproteins; HDL: High-density lipoproteins. The figure was retrieved from Servier Medical Art (http://smart.servier.com/).

The first step in lipoprotein metabolism, called the exogenous pathway, consists of the absorption of dietary lipids in the intestine, followed by their incorporation into chylomicrons. The TAGs present in chylomicrons are subsequently hydrolyzed by lipoprotein lipase (LPL) to form chylomicron remnants, which are taken up by the liver. The second step, the endogenous pathway, begins with the formation of very low-density lipoproteins (VLDL) in the liver. Via the activity of the LPL, TAGs present in VLDL are hydrolyzed to form intermediate-density lipoproteins (IDL) and finally LDL, which are taken up by various organs by binding to the LDL-R. The final step, i.e. the reverse cholesterol transport pathway (RCT), allows the transfer of free cholesterol from peripheral tissues to discoidal lipid-poor apolipoprotein A-I (apoA-I), i.e. pre $\beta-1$ HDL. Free cholesterol is then esterified within the HDL particle by lecithin:cholesterol acyltransferase (LCAT) to form mature HDL particles rich in CE. As such, HDLs are involved in the transport of cholesterol from peripheral tissues back to the liver, where CE binds to the scavenger receptor class B member I 
receptor (SR-BI). In the liver, CE are hydrolyzed and can be directly excreted as free cholesterol into the bile, or converted into bile acids by the cholesterol 7alphahydroxylase (CYP7A1) and then excreted from the body. CE can also follow an alternative pathway and be exchanged for TAG from HDLs to apoB100-containing lipoproteins (VLDLs, IDLs and LDLs), via the activity of the plasma glycoprotein called cholesteryl ester transfer protein (CETP) (Figure 3) [14, 15]. In this thesis we will focus on determinants of HDL functionality.

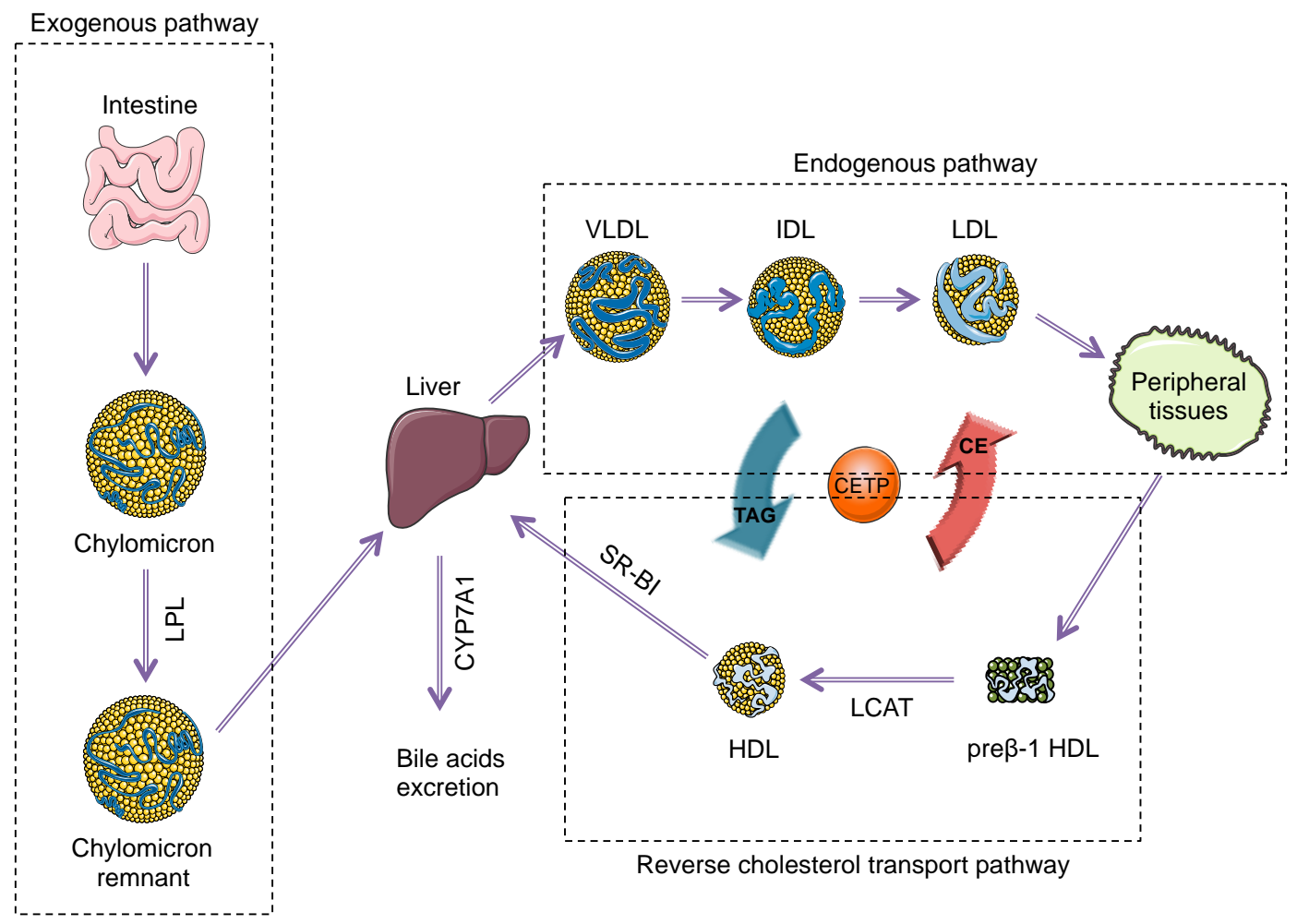

Figure 3: Lipoproteins metabolism.

LPL: Lipoprotein lipase; CE: Cholesteryl ester; TAG: Triacylglycerol; CETP: cholesteryl ester transfer protein; LCAT: Lecithin:cholesterol acyltransferase; SR-BI: Scavenger receptor class B member I; CYP7A1: Cholesterol 7alpha-hydroxylase. VLDL: Very low-density lipoproteins; IDL: Intermediate-density lipoproteins; LDL: Lowdensity lipoproteins; HDL: High-density lipoproteins. The figure was produced using Servier Medical Art (http://smart.servier.com/). 


\section{HDL particles characteristics}

HDL are the densest lipoproteins and consist of different particle subpopulations, which are heterogeneous in structure, density, size, composition, and biological activity [16].

\section{Size}

HDL particles are synthesized as small, lipid-poor, discoid particles, called pre $\beta-1$ HDL, which are rich in unesterified cholesterol [17]. Free cholesterol present in these discoidal HDL particles is converted into CE. Because CE is stored into HDL particles' core, this leads to the formation of bigger and spherical particles. The LCAT enzyme esterifies cholesterol and together with the phospholipid transfer protein (PLTP), larger HDL3 and finally mature HDL2 particles are formed, rich in $\mathrm{CE}$, which account for the major proportion of HDLs in plasma [18].

\section{Composition}

Approximately half of the total HDL mass is due to its lipids content, with phospholipids accounting for $40-60 \%$ of the total lipids, followed by CE $(30-40 \%)$, TAG (5-12\%), and free cholesterol (5-10\%) [19]. The other half consists of proteins. ApoA-I is the main apolipoprotein of HDL and accounts for $70 \%$ of the total protein content. The atheroprotective properties of HDL are mainly attributed to apoA-I [20]. HDL also contains a variety of other proteins, such as apoA-II, apoA-IV, apo CI, apo C-II, apo C-III, apoD, apoE, apoJ, apo L-I, apoM, serum amyloid A (SAA) proteins, ceruloplasmin, transferrin, and enzymes including LCAT, PON1, and PAF-AH/Lp-PLA2 [21, 22]. However, the precise role of these various other apolipoproteins, either alone or in specific combinations, in HDL metabolism are not fully understood and warrants further investigations [22].

\section{HDL functionality}

HDL particles possess many anti-atherogenic properties, such as anti-inflammatory, anti-oxidant, anti-apoptotic, anti-thrombotic and vasodilating effects [10, 23]. The suggested most anti-atherogenic property of HDL is its ability to promote cholesterol efflux via the RCT. In fact, the cholesterol efflux capacity of HDL particles is negatively associated with the development of CVD [24], emphasizing the importance of measuring HDL-efflux capacity rather than simply analyzing circulation serum HDL-C concentrations. 
Cholesterol efflux from cholesterol-laden macrophages is facilitated by specific transporters such as adenosine triphosphate binding cassette transporter A1 (ABCA1), adenosine triphosphate binding cassette transporter G1 (ABCG1), and scavenger receptor $\mathrm{BI}$ (SR-BI). ABCA1 promotes the transfer of free cholesterol from peripheral tissues and macrophages, to pre $\beta-1$ HDL, whereas ABCG1 and SR-BI transport cholesterol to mature HDL, i.e. HDL2 and HDL3 [15]. The individual contribution of each transporter-mediated efflux pathway has been estimated by using macrophages from mice in which these specific transporters have been knockout [25]. Based on these experiments, it has been estimated that $35 \%$ of the efflux is attributed to the ABCA1 transporter, underlining that it plays a major role in total cholesterol efflux. ABCG1 accounted for approximately $20 \%$ of the efflux, while SR-BI contributed for only $9 \%$ [15, 25]. Another unmediated pathway involved in the efflux of cholesterol is aqueous diffusion. This mechanism is driven by the cholesterol concentration gradient itself, and consists of a passive diffusion process between mature $\mathrm{HDL}_{2}$ and $\mathrm{HDL}_{3}$ particles, and cells [26-28]. Aqueous diffusion has been demonstrated to account for $35 \%$ of the total efflux [25].

The anti-atherogenic properties of each HDL subfractions are described in Figure 4.

As apoA-I is the main functional protein within HDL, increasing apoA-I synthesis might be a potential target in order to improve HDL functionality. High concentrations of apoA-I have been associated with a lower CVD risk [29], and apoA-I concentrations have been positively associated with cholesterol efflux [24, 30]. However, the concentration of apoA-I does not fully reflect HDL-efflux capacity and individuals with similar levels of apoA-I can display different abilities to promote cholesterol efflux [31]. Thus, it appears that cholesterol efflux capacity of HDL particles is regulated by a variety of factors. 


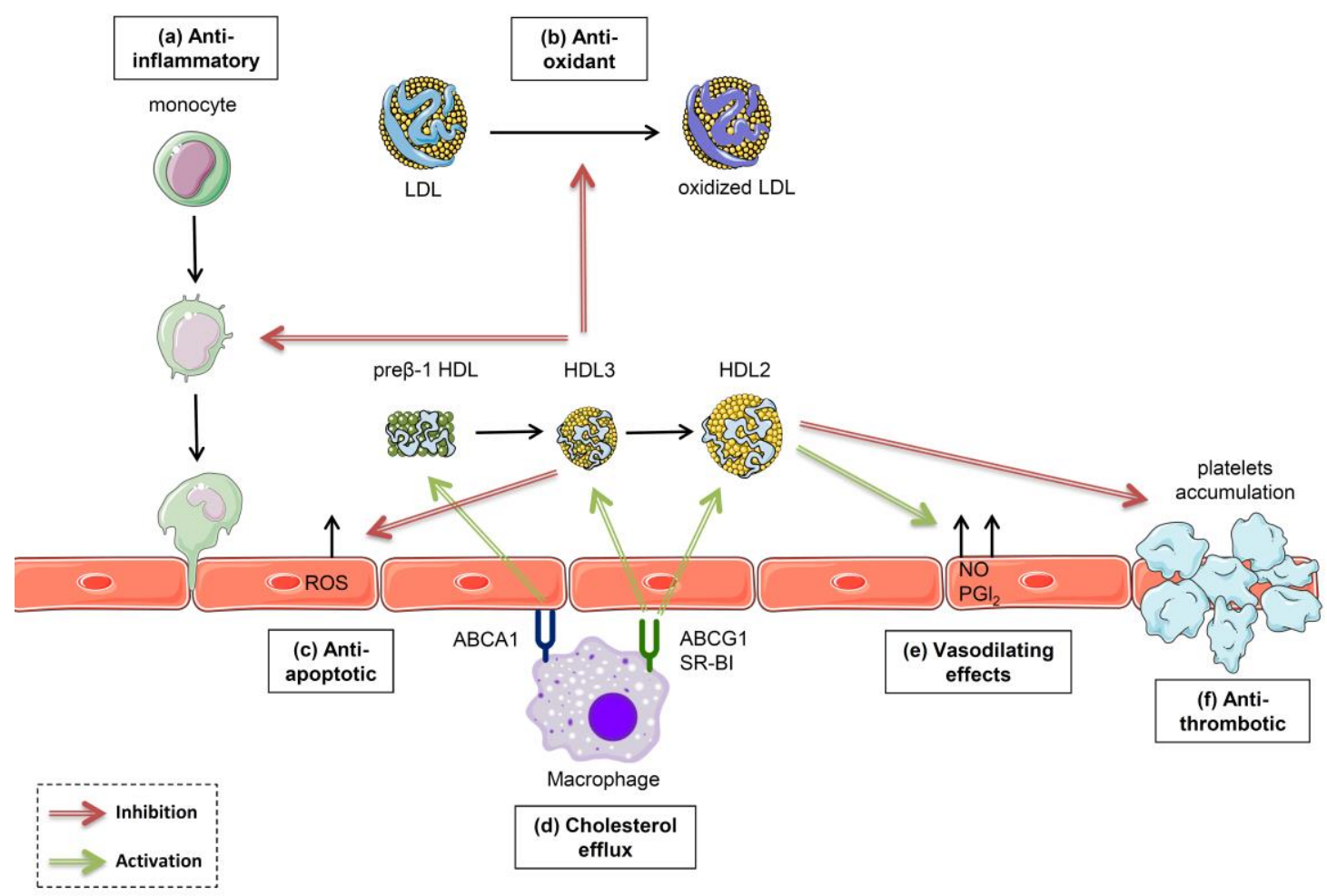

Figure 4: Anti-atherogenic properties of HDL particles. Adapted from Badimon et Vilahur [3] and Camont et al. [16]. The figure was produced using Servier Medical Art (http://smart.servier.com/).

(a) Anti-inflammatory effects by inhibiting the adhesion of monocyte in the endothelium; (b) Anti-oxidant effects by protecting LDL from oxidation; (c) Anti-apoptotic effects by preventing the production of reactive oxygen species (ROS); (d) Cholesterol efflux: ABCA1 transporter promotes the transfer of free cholesterol from peripheral tissues to discoidal lipid-poor apoA-I, i.e. pre $\beta-1 \mathrm{HDL}$, while ABCG1 and SR-BI contribute to the efflux of cholesterol to mature HDL particles; (e) Vasodilating effects by stimulating the production of nitric oxide (NO) and prostacyclin $\left(\mathrm{PGl}_{2}\right) ;(f)$ Anti-thrombotic effects via the inhibition of platelets accumulation [3, 16].

\section{Modulators of cholesterol efflux}

Characteristics of the HDL particle itself, such as the size and the composition, can modulate HDL cholesterol efflux capacity. Furthermore, individuals' characteristics, for instance BMI, gender, age and the genetic background, as well as nutrition and lifestyle are also important modulators of cholesterol efflux capacity. The associations between these factors and cholesterol efflux have been systematically reviewed and are discussed into detail in chapter 2 of this thesis. In addition, it has been recently shown that HDL particles are carrying microRNAs (miRNAs), and transport and deliver these miRNAs to recipient cells [32]. 
MiRNA are small, non-coding RNAs of approximately 22 nucleotides that bind to complementary sites in the $3^{\prime}$ UTR region of the target messenger RNAs (mRNAs), and inhibit gene expression through post-transcriptional regulation [33-35]. The cellular release mechanisms and extracellular transport of miRNAs are complex, and described in Figure 5.

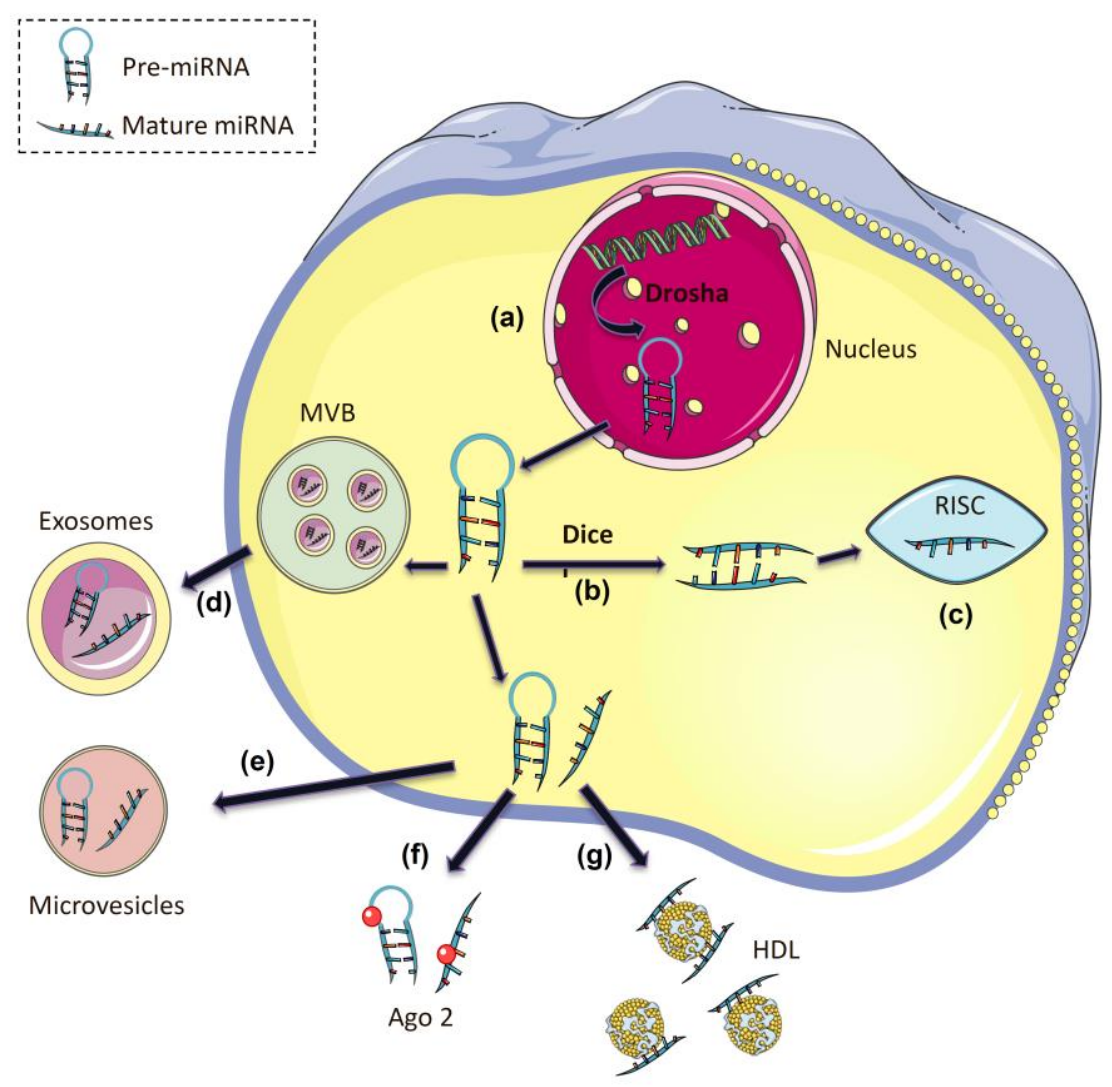

Figure 5: Cellular release mechanisms of miRNAs. Adapted from Creemers et al. [36]. The figure was produced using Servier Medical Art (http://smart.servier.com/).

(a) In the nucleus, DNA is cleaved by the RNase III enzyme Drosha into a pre-miRNA (70 nts); (b) After being transported into the cytoplasm by the Exportin-5 protein, this pre-miRNA is cleaved by Dicer, which is an endoribonuclease of the RNase III family, into a mature miRNA (22 nts); (c) One strand of this mature miRNA can be loaded into the RNA-induced silencing complex (RISC), where it can guide the RISC complex to specific mRNA targets to prevent translation of the mRNA into protein. The other strand is likely subject to degradation but it can also be released from the cell through several mechanisms; (d) In the cytoplasm, pre-miRNAs or mature miRNAs can be incorporated into small vesicles called exosomes, which are released from the cells after fusion of multivesicular bodies (MVB) with the plasma membrane; (e) They can also be released by microvesicles through budding of the plasma membrane; (f) Finally, miRNAs can be found in circulation in microparticle-free form and bind to RNA-binding proteins such as Argonaute2 (Ago2); (g) or be associated with HDL particles [36]. 
MiRNAs are expressed in all eukaryotic cells types, and coordinate key cellular processes including proliferation, differentiation, and apoptosis [37]. In addition, a single miRNA can target and regulate numerous mRNAs, while a single mRNA can be regulated by multiple miRNAs [38]. HDL-miRNAs have been associated with several disorders, such as hypercholesterolemia, inflammation and acute coronary syndrome [32, 39], and the HDL-miRNAs signature is altered in diseases [32, 39, 40] (Table 1).

Table 1: HDL-associated miRNAs levels in diseases [41].

\begin{tabular}{|c|c|c|c|}
\hline $\begin{array}{c}\text { HDL- } \\
\text { miRNAs }\end{array}$ & Disease & $\begin{array}{c}\text { Effect on HDL-miRNAs } \\
\text { level }\end{array}$ & References \\
\hline let-7f-5p & Familial hypercholesterolemia & $\uparrow$ & [32] \\
\hline miR-24-3p & Familial hypercholesterolemia & $\uparrow$ & [32] \\
\hline $\operatorname{miR}-30 c-5 p$ & Acute coronary syndrome & $\downarrow$ & [39] \\
\hline \multirow[t]{2}{*}{$\operatorname{miR-92a-3p}$} & Acute coronary syndrome & $\downarrow$ & [39] \\
\hline & $\begin{array}{l}\text { Stable angina; Unstable angina; } \\
\text { Myocardial infarction }\end{array}$ & 个 in $\mathrm{HDL}_{2}$ and $\mathrm{HDL}_{3}$ & [40] \\
\hline miR-106a-5p & Familial hypercholesterolemia & $\uparrow$ & [32] \\
\hline $\operatorname{miR}-122-5 p$ & Stable angina; Unstable angina & $\uparrow$ in $\mathrm{HDL}_{3}$ & [40] \\
\hline miR-135a-3p & Familial hypercholesterolemia & $\downarrow$ & [32] \\
\hline $\operatorname{miR}-138-1-3 p$ & Familial hypercholesterolemia & $\downarrow$ & [32] \\
\hline \multirow[t]{2}{*}{ miR-146a-5p } & Acute coronary syndrome & $\downarrow$ & [39] \\
\hline & $\begin{array}{l}\text { Unstable angina; Myocardial } \\
\text { infarction }\end{array}$ & $\uparrow$ in $\mathrm{HDL}_{3}$ & [40] \\
\hline miR-188-5p & Familial hypercholesterolemia & $\downarrow$ & [32] \\
\hline $\operatorname{miR}-191-5 p$ & Familial hypercholesterolemia & $\uparrow$ & [32] \\
\hline miR-218-5p & Familial hypercholesterolemia & $\uparrow$ & [32] \\
\hline $\operatorname{miR}-222-3 p$ & Familial hypercholesterolemia & $\uparrow$ & [32] \\
\hline miR-223-3p & Familial hypercholesterolemia & $\uparrow$ & [32] \\
\hline miR-323-3p & Familial hypercholesterolemia & $\downarrow$ & [32] \\
\hline $\operatorname{miR}-342-3 p$ & Familial hypercholesterolemia & $\uparrow$ & [32] \\
\hline $\operatorname{miR}-412-5 p$ & Familial hypercholesterolemia & $\uparrow$ & [32] \\
\hline $\operatorname{miR}-486-5 p$ & $\begin{array}{l}\text { Stable angina; Unstable angina; } \\
\text { Myocardial infarction }\end{array}$ & $\uparrow$ in $\mathrm{HDL}_{2}$ & [40] \\
\hline miR-509-3p & Familial hypercholesterolemia & $\downarrow$ & [32] \\
\hline $\operatorname{miR}-520 c-3 p$ & Familial hypercholesterolemia & $\downarrow$ & [32] \\
\hline miR-572 & Familial hypercholesterolemia & $\downarrow$ & [32] \\
\hline miR-573 & Familial hypercholesterolemia & $\downarrow$ & [32] \\
\hline $\operatorname{miR}-625-3 p$ & Familial hypercholesterolemia & $\downarrow$ & [32] \\
\hline miR-632 & Familial hypercholesterolemia & $\downarrow$ & [32] \\
\hline miR-877-5p & Familial hypercholesterolemia & $\uparrow$ & [32] \\
\hline
\end{tabular}


As shown in the Table 1, expression of miRNAs can be either upregulated or downregulated in metabolic diseases; however it is currently difficult to know if these changes in HDL-miRNAs are a cause or a consequence of the diseases. In this context, the association of specific HDL-miRNAs with various diseases, as well as miRNAs-based therapy using miRNAs antagonists or miRNAs mimics, could serve as useful biomarker but also as a target for the prevention and the treatments of CVD [41]. In addition, the miRNAs associated with HDL particles may be involved in the regulation of the HDL biological functions. However, their specific role is not well known yet. One of the most studied HDL-associated miRNA is miR-223, which is also one the most abundant miRNA associated with HDL particles [32, 39]. MiR-223 has been recently found to coordinates cholesterol homeostasis, by controlling cholesterol biosynthesis, uptake and efflux [42]. However, the proportion of miRNAs associated with HDL particles is rather small, and HDL-miR-223 was found to contribute to only $8 \%$ of the total miR-223 in the circulation [39]. In addition, miR-223 is also one of the most abundant miRNA present in LDL. However, miR223 levels in LDL particles are approximately 7 times lower as compared to miR-223 levels in HDL particles [39]. Finally, miR-223 is also present in exosomes, and exosomes-miR-223 levels is increased in acute ischemic stroke patients and positively associated with stroke occurrence and severity [43]. However, when studied in the same subject, the exosomes-miRNAs signature was found to be more closely associated with the LDL-miRNAs signature than with the HDL-miRNAs [32]. Because of the growing interest in miRNAs research, an organized classification (i.e. miRandola) regrouping all the available information on circulating miRNA has been developed $[44,45]$.

\section{Thesis outline}

The aim of this thesis was to investigate how HDL functionality, i.e. HDL cholesterol efflux capacity, can be modulated and improved in humans, in order to ultimately reduce CVD risk. It is first essential to better understand factors associated with HDL-mediated cholesterol efflux. For this, a review was written (chapter 2), which summarizes determinants of cholesterol efflux in humans. It was concluded that results from studies in which efflux was measured seem to be in contradiction with results from epidemiological studies, and thus that more research is needed in order to determine the best targets to improve efflux capacity. Next, chapter 3 discusses the association between genetic determinants and cholesterol efflux, in healthy volunteers. In chapter $\mathbf{4}$, the involvements of three specific HDL-associated microRNAs on HDL-mediated cholesterol efflux were studied. Furthermore, in chapters 4 and 5, the effects of three dietary compounds have been investigated. Chapter 4 focuses on the effects of theobromine consumption on fasting and 
postprandial cholesterol efflux, while chapter 5 describes the effects of sterol and stanol supplementation on fasting HDL-mediated cholesterol efflux. Chapter 6 focuses on the effect of diet-induced weight loss on HDL cholesterol efflux in abdominally obese men. Clinical differences between lean and obese individuals are also discussed in this chapter. Finally, chapter $\mathbf{7}$ summarizes the major findings of the different studies presented in this thesis, and the results are discussed into broader perspective. 


\section{References}

1. Organization, W.H. Cardiovascular diseases (CVDs) fact sheet No. 317. September 2016; Available from: http://www.who.int/mediacentre/factsheets/fs317/en/.

2. Frostegard, J., Immunity, atherosclerosis and cardiovascular disease. BMC Med, 2013. 11: p. 117.

3. Badimon, L. and G. Vilahur, LDL-cholesterol versus HDL-cholesterol in the atherosclerotic plaque: inflammatory resolution versus thrombotic chaos. Ann N Y Acad Sci, 2012. 1254: p. 18-32.

4. Stein, E.A., Low-density lipoprotein cholesterol reduction and prevention of cardiovascular disease. Mayo Clin Proc, 2009. 84(4): p. 307-9.

5. Stroes, E., Statins and LDL-cholesterol lowering: an overview. Curr Med Res Opin, 2005. 21 Suppl 6: p. S9-16.

6. Ebrahim, S., F.C. Taylor, and P. Brindle, Statins for the primary prevention of cardiovascular disease. Bmj, 2014. 348: p. g280.

7. Boekholdt, S.M., et al., Very low levels of atherogenic lipoproteins and the risk for cardiovascular events: a meta-analysis of statin trials. J Am Coll Cardiol, 2014. 64(5): p. 485-94.

8. Blom, D.J., et al., PCSK9 inhibition in the management of hyperlipidemia: focus on evolocumab. Vasc Health Risk Manag, 2016. 12: p. 185-97.

9. Sabatine, M.S., et al., Evolocumab and Clinical Outcomes in Patients with Cardiovascular Disease. N Engl J Med, 2017. 376(18): p. 1713-1722.

10. Barter, P., et al., High density lipoproteins (HDLs) and atherosclerosis; the unanswered questions. Atherosclerosis, 2003. 168(2): p. 195-211.

11. Choi, B.G., et al., The role of high-density lipoprotein cholesterol in the prevention and possible treatment of cardiovascular diseases. Curr Mol Med, 2006. 6(5): p. 571-87.

12. Rader, D.J. and G.K. Hovingh, HDL and cardiovascular disease. Lancet, 2014. 384(9943): p. 618-25.

13. Ronsein, G.E. and J.W. Heinecke, Time to ditch HDL-C as a measure of HDL function? Curr Opin Lipidol, 2017. 28(5): p. 414-418.

14. Feingold, K.R. and C. Grunfeld, Introduction to Lipids and Lipoproteins, in Endotext, L.J. De Groot, et al., Editors. 2000, MDText.com, Inc.: South Dartmouth (MA).

15. Tall, A.R., Cholesterol efflux pathways and other potential mechanisms involved in the athero-protective effect of high density lipoproteins. J Intern Med, 2008. 263(3): p. 256-73.

16. Camont, L., M.J. Chapman, and A. Kontush, Biological activities of HDL subpopulations and their relevance to cardiovascular disease. Trends Mol Med, 2011. 17(10): p. 594-603.

17. Rye, K.A. and P.J. Barter, Formation and metabolism of prebeta-migrating, lipid-poor apolipoprotein A-I. Arterioscler Thromb Vasc Biol, 2004. 24(3): p. 421-8.

18. Zannis, V.I., et al., HDL biogenesis, remodeling, and catabolism. Handb Exp Pharmacol, 2015. 224: p. 53-111.

19. Kontush, A., M. Lhomme, and M.J. Chapman, Unraveling the complexities of the HDL lipidome. J Lipid Res, 2013. 54(11): p. 2950-63. 
20. Millar, J.S. and M. Cuchel, ApoA-I-Directed Therapies for the Management of Atherosclerosis. Curr Atheroscler Rep, 2015. 17(10): p. 60.

21. Lewis, G.F. and D.J. Rader, New insights into the regulation of HDL metabolism and reverse cholesterol transport. Circ Res, 2005. 96(12): p. 1221-32.

22. Karavia, E.A., et al., HDL quality and functionality: what can proteins and genes predict? Expert Rev Cardiovasc Ther, 2014. 12(4): p. 521-32.

23. Rye, K.A., et al., The metabolism and anti-atherogenic properties of HDL. J Lipid Res, 2009. 50 Suppl: p. S195-200.

24. Khera, A.V., et al., Cholesterol efflux capacity, high-density lipoprotein function, and atherosclerosis. N Engl J Med, 2011. 364(2): p. 127-35.

25. Adorni, M.P., et al., The roles of different pathways in the release of cholesterol from macrophages. J Lipid Res, 2007. 48(11): p. 2453-62.

26. Yancey, P.G., et al., Importance of different pathways of cellular cholesterol efflux. Arterioscler Thromb Vasc Biol, 2003. 23(5): p. 712-9.

27. Zhao, Y., T.J. Van Berkel, and M. Van Eck, Relative roles of various efflux pathways in net cholesterol efflux from macrophage foam cells in atherosclerotic lesions. Curr Opin Lipidol, 2010. 21(5): p. 441-53.

28. Phillips, M.C., et al., Mechanisms of high density lipoprotein-mediated efflux of cholesterol from cell plasma membranes. Atherosclerosis, 1998. 137 Suppl: p. S13-7.

29. Ding, D. and X. Li, Serum lipids, apolipoproteins, and mortality among coronary artery disease patients. 2014. 2014: p. 709756.

30. Borja, M.S., et al., HDL-apolipoprotein A-I exchange is independently associated with cholesterol efflux capacity. J Lipid Res, 2015. 56(10): p. 2002-9.

31. de la Llera-Moya, M., et al., The ability to promote efflux via ABCA1 determines the capacity of serum specimens with similar high-density lipoprotein cholesterol to remove cholesterol from macrophages. Arterioscler Thromb Vasc Biol, 2010. 30(4): p. 796-801.

32. Vickers, K.C., et al., MicroRNAs are transported in plasma and delivered to recipient cells by high-density lipoproteins. Nat Cell Biol, 2011. 13(4): p. 423-33.

33. Lai, E.C., Micro RNAs are complementary to 3' UTR sequence motifs that mediate negative post-transcriptional regulation. Nat Genet, 2002. 30(4): p. 363-4.

34. Jackson, R.J. and N. Standart, How do microRNAs regulate gene expression? Sci STKE, 2007. 2007(367): p. re1.

35. Bartel, D.P., MicroRNAs: target recognition and regulatory functions. Cell, 2009. 136(2): p. 215-33.

36. Creemers, E.E., A.J. Tijsen, and Y.M. Pinto, Circulating microRNAs: novel biomarkers and extracellular communicators in cardiovascular disease? Circ Res, 2012. 110(3): p. 48395.

37. Turchinovich, A., et al., Circulating miRNAs: cell-cell communication function? Front Genet, 2013. 4: p. 119.

38. Shu, J., et al., Dose-dependent differential mRNA target selection and regulation by let-7a$7 f$ and miR-17-92 cluster microRNAs. RNA Biol, 2012. 9(10): p. 1275-87.

39. Wagner, J., et al., Characterization of levels and cellular transfer of circulating lipoproteinbound microRNAs. Arterioscler Thromb Vasc Biol, 2013. 33(6): p. 1392-400. 
40. Niculescu, L.S., et al., MiR-486 and miR-92a Identified in Circulating HDL Discriminate between Stable and Vulnerable Coronary Artery Disease Patients. PLoS One, 2015. 10(10): p. e0140958.

41. Desgagne, V., L. Bouchard, and R. Guerin, microRNAs in lipoprotein and lipid metabolism: from biological function to clinical application. Clin Chem Lab Med, 2017. 55(5): p. 667-686.

42. Vickers, K.C., et al., MicroRNA-223 coordinates cholesterol homeostasis. Proc Natl Acad Sci U S A, 2014. 111(40): p. 14518-23.

43. Chen, Y., et al., Increased Circulating Exosomal miRNA-223 Is Associated with Acute Ischemic Stroke. Front Neurol, 2017. 8: p. 57.

44. Russo, F., et al., miRandola: extracellular circulating microRNAs database. PLoS One, 2012. 7(10): p. e47786.

45. Russo, F., et al., miRandola 2017: a curated knowledge base of non-invasive biomarkers. Nucleic Acids Res, 2017. 


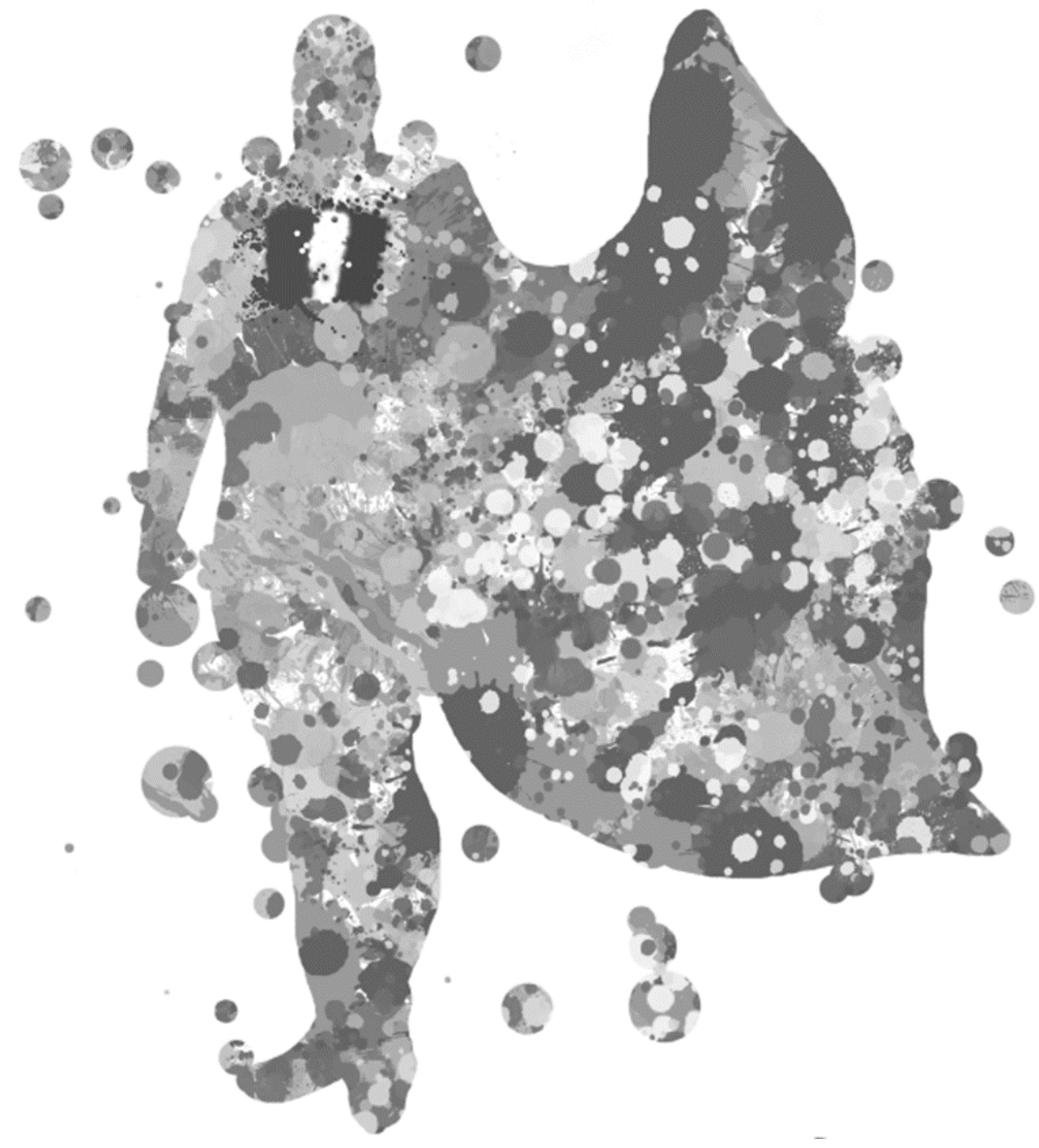




\section{Chapter 2}

\section{Determinants of cholesterol efflux capacity in humans}

Charlotte P.J. Talbot, Jogchum Plat, Andreas Ritsch, Ronald P. Mensink

Progress in Lipid Research. 2017; 69: 21-32

Progress in Lipid Research. 2017; 69: 21-32 


\begin{abstract}
High-density lipoproteins (HDL) play an important role in the reverse cholesterol transport (RCT) pathway, contributing to the efflux of excess cellular cholesterol. However, the classic hypothesis that raising HDL-cholesterol (HDL-C) concentrations reduces cardiovascular disease (CVD) risk has been challenged by recent intervention studies. Nowadays, improving cholesterol efflux capacity of HDL particles is considered to be a better target for the prevention of CVD. We will first briefly summarize assays that have been developed in order to quantify HDLmediated cholesterol efflux from macrophages. However, the main purpose of this review is to discuss factors and ABC-transporters that are associated with HDLmediated cholesterol efflux, such as HDL particle characteristics (i.e. HDL size and composition), subjects' characteristics (i.e. gender, BMI and age), HDL-C raising drugs, lifestyle, genetic background, as well as acute and low-grade systemic inflammation. Results suggested that factors associated with small HDL particles efficiently promote cholesterol efflux via the ABCA1 transporter. This appears to contradict findings from epidemiological studies suggesting that in particular large HDL2 particles are related to a reduced CVD risk. It is therefore essential not only to understand targets to increase cholesterol efflux capacity, but also to prove causality between cholesterol efflux capacity and the prevention of CVD risk.
\end{abstract}




\section{Introduction}

Cardiovascular disease (CVD) is the principal cause of death worldwide. It is generally accepted that decreasing serum low-density lipoprotein cholesterol concentrations reduces CVD risk, but the role of high-density lipoprotein cholesterol (HDL-C) is controversial. Numerous epidemiological studies have observed an inverse relationship between serum HDL-C concentrations and CVD risk [1-3], but actively raising serum HDL-C concentrations did not reduce CVD risk, challenging the classic HDL-C hypothesis [4].

HDL particles have been shown to exert numerous anti-atherogenic properties, including anti-inflammatory, anti-oxidant, anti-apoptotic, anti-thrombotic and vasodilating effects. However, a major postulated anti-atherogenic property of HDL is its ability to promote cholesterol efflux from macrophages, which is part of the reverse cholesterol transport (RCT) pathway [5]. An inverse relationship between in vitro HDL-mediated cholesterol efflux from macrophages with CVD risk has been demonstrated, independently of HDL-C and apolipoprotein A-I (apoA-I) concentrations [6,7], as well as with cardiovascular mortality [8]. In fact, when adjusted for cholesterol efflux capacity, the inverse relationship between HDL-C and apoA-I concentrations with CVD disappeared [7]. These findings suggest that improving HDL functionality is a better target for decreasing CVD risk than aiming for increased fasting HDL-C or apoA-I concentrations.

In this review, we will first briefly summarize the RCT pathway and several assays that have been developed to quantify HDL-mediated cholesterol efflux from macrophages. The main focus, however, is to discuss factors that may be related to HDL-mediated cholesterol efflux, as related to changes in HDL particle characteristics.

\section{Reverse Cholesterol Transport}

The RCT pathway is a complex process resulting in the transport of cholesterol from peripheral tissues back to the liver for excretion into bile as either free cholesterol or bile acids. A simplified scheme is shown in Figure 1. 


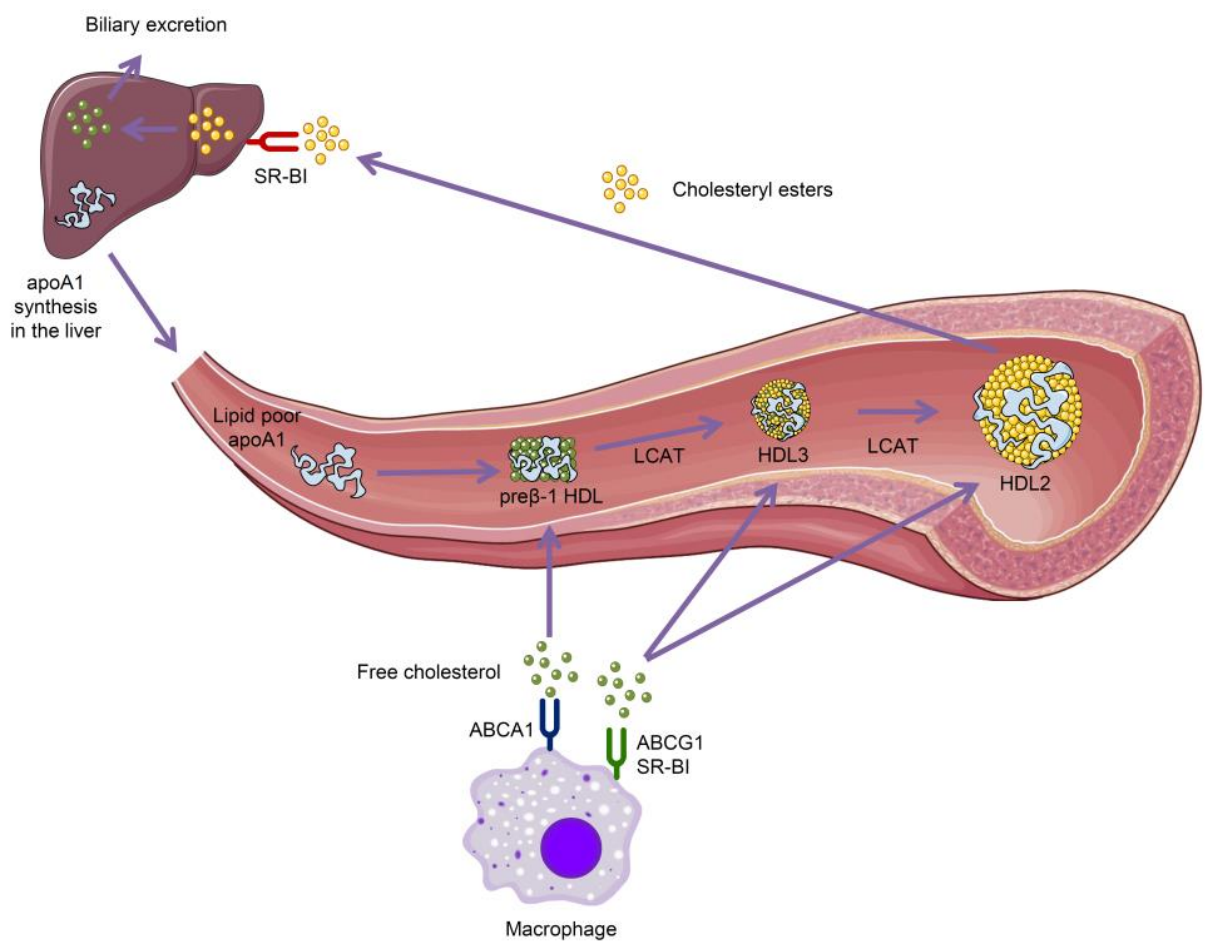

Figure 1: HDL metabolism and reverse cholesterol transport (RCT) pathway. Figure was produced using Servier Medical Art (http://smart.servier.com/).

HDL: High-density lipoproteins; ABCA1: ATP-binding cassette 1; ABCG1: ATP-binding cassette sub-family G member 1; SR-BI: Scavenger receptor class B member I; LCAT: Lecithin:cholesterol acyltransferase.

The ATP-binding cassette 1 (ABCA1) transporter promotes the transfer of free cholesterol from peripheral tissues to discoidal lipid-poor apoA-I, i.e. pre $\beta-1$ HDL. Free cholesterol is then esterified by lecithin:cholesterol acyltransferase (LCAT), and larger spherical $\mathrm{HDL}_{3}$ and finally $\mathrm{HDL}_{2}$ particles are formed. The ATP-binding cassette G1 transporter (ABCG1) and scavenger receptor class B type I (SR-BI) also contribute to the efflux of cholesterol from peripheral tissues and macrophages to mature HDL particles [5, 9]. The HDL2 particles bind to hepatocytes through SR-BI, which mediates the uptake of cholesteryl esters (CE) from HDL to the liver. In the liver, CE are hydrolyzed by cholesteryl ester hydrolase and excreted as free cholesterol or converted into bile acids, mostly via the action of cholesterol 7 alphahydroxylase (CYP7A1), the rate-limiting enzyme in the catabolism of cholesterol into bile acids [5, 9-11]. CE can also follow an alternative pathway involving the exchange of CE from HDLs for triglyceride (TG) to apoB100-containing lipoproteins 
(very-low density lipoproteins (VLDLs), intermediate-density lipoproteins (IDLs), and LDLs), resulting in the remodeling of the composition, size, and, consequently, the function of lipoproteins. This exchange of CE for TG requires the activity of cholesteryl ester transfer protein (CETP). Via this pathway, extrahepatic cholesterol is ultimately taken up by the liver through the LDL receptor $[2,5,12-14]$. Recently, a third pathway, called trans-intestinal excretion of cholesterol (TICE), has been proposed. This pathway is involved in the excretion of peripheral and hepatic cholesterol into the intestinal lumen, independent of the biliary route [15]. However, it is not fully understood which particles transport the cholesterol to the intestine and how these particles are internalized, although studies in mice have suggested that HDL does not contribute to TICE [16, 17] (Figure 2).

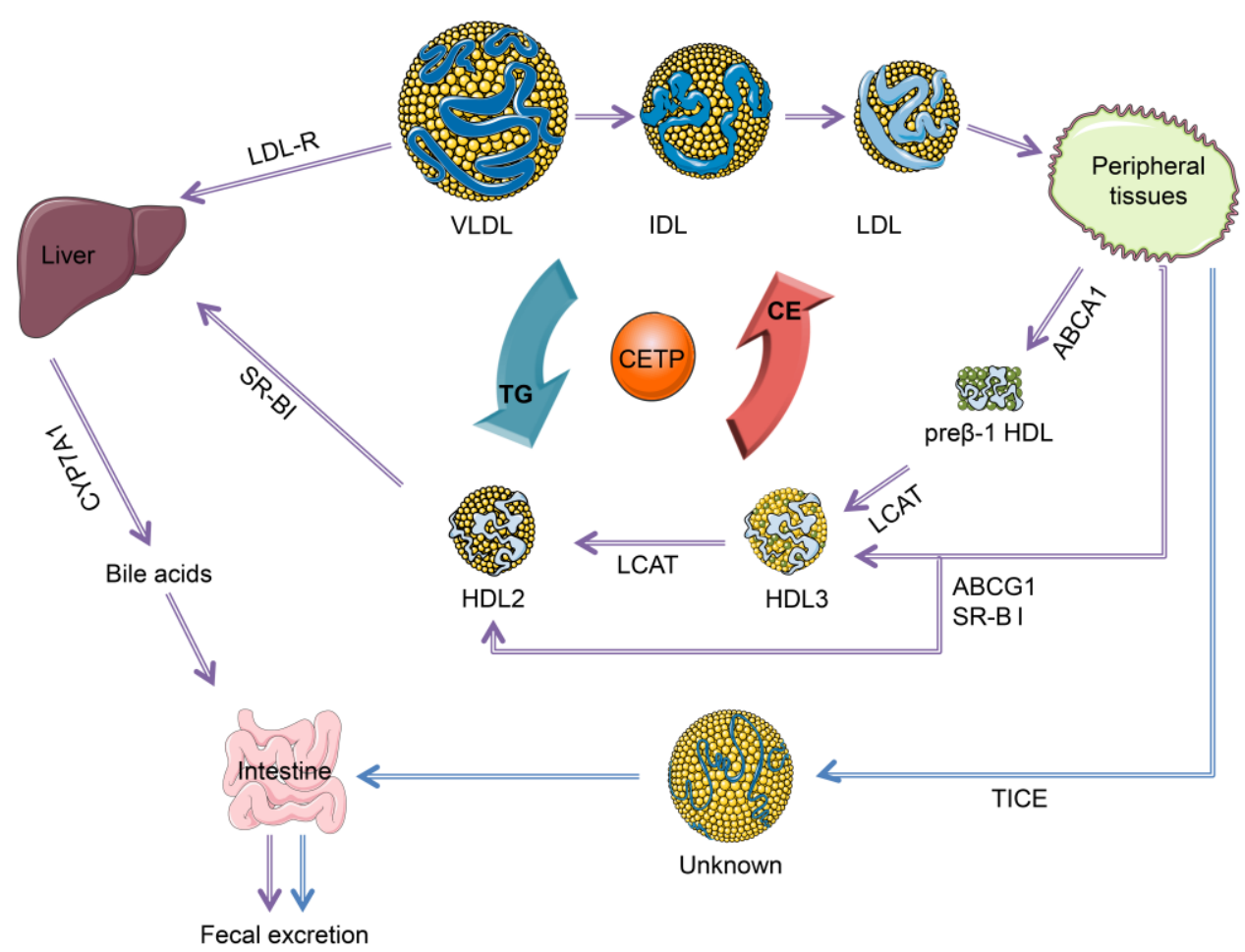

Figure 2: CETP activity. Figure was produced using Servier Medical Art (http://smart.servier.com/).

HDL: High-density lipoproteins; IDL: Intermediate-density lipoproteins; LDL: Low-density lipoproteins; VLDL: Very low-density lipoproteins; ABCA1: ATP-binding cassette 1; ABCG1: ATP-binding cassette sub-family G member 1; CE: cholesteryl esters; TG: triglyceride; CETP: cholesteryl ester transfer protein; LCAT: Lecithin:cholesterol acyltransferase; SR-BI: Scavenger receptor class B member I; CYP7A1: cholesterol 7alphahydroxylase; TICE: trans-intestinal cholesterol excretion. 
The importance of ABCA1 transporter for cholesterol efflux from macrophages has been demonstrated in many studies. For example, individual differences in cholesterol efflux capacity from macrophages are predominantly due to the ABCA1mediated pathway [18]. Moreover, Tangier disease is caused by mutations in ABCA1 and characterized by a reduced cholesterol efflux, low HDL-C and apoA-I concentrations, and an increased risk to develop atherosclerosis [13]. ABCA1 knockout mice have a similar phenotype [19]. Therefore, the RCT, and more specifically activating the ABCA1-mediated pathway, may be an attractive target for the prevention and regression of atherosclerosis.

\section{Methodology of HDL cholesterol efflux measurements}

Several cholesterol efflux assays have been developed to quantify cholesterol efflux from cultivated cells to an extracellular cholesterol acceptor. In general, donor cells (e.g. monocytes, macrophages) are first labeled with ${ }^{3} \mathrm{H}$-cholesterol [20]. However, deuterated cholesterol, a stable isotope or a fluorescent-labeled sterol, BODIPYcholesterol, have also been used [21]. Results of studies using BODIPY-cholesterol as label correlated modestly with those using ${ }^{3} \mathrm{H}$-cholesterol $(\mathrm{r}=0.54 ; \mathrm{n}=179)$, while fractional efflux rates using BODIPY-cholesterol were approximately 3 times greater than with ${ }^{3} \mathrm{H}$-cholesterol [22]. Another method uses the fluorescent Pennsylvania Green/N-alkyl-3 $\beta$-cholesterylamine-derived molecular probe $(\mathrm{F}-\mathrm{Ch})$ and changes in cholesterol efflux capacity induced in vitro by pharmacologic agents were comparable to those of the ${ }^{3} \mathrm{H}$-cholesterol method. Although the kinetics of the F-Ch and ${ }^{3} \mathrm{H}$-cholesterol assays were comparable, i.e. a steady increase and a peak value reached at $24 \mathrm{~h}$, efflux values using $\mathrm{F}-\mathrm{Ch}$ were lower compared to those using ${ }^{3} \mathrm{H}$ cholesterol, irrespective whether apoA-I or HDL were used as acceptors [23]. Fluorescent probes are therefore a good alternative to avoid the detection and disposal of radioactivity, inherent to an assay using ${ }^{3} \mathrm{H}$-cholesterol. Another approach using a fluorescent enzymatic method has been described to quantify the cholesterol mass in macrophages. With this method, cholesterol efflux can be indirectly calculated by quantifying the fluorescence remaining in the cells [24]. An in vivo assay in humans has also been developed and consists of infusion of a stable isotope, i.e. ${ }^{13} \mathrm{C} 2$-cholesterol. As compared to the in vitro assays, three main steps of the RCT pathway can be measured with this in vivo assay: the efflux of free cholesterol from tissues, the esterification of free cholesterol in plasma, and fecal sterol excretion $[25,26]$. If efflux estimates from this in vivo assay correlate with those of in vitro assays remain to be determined. A novel in vivo approach using ${ }^{3} \mathrm{H}$ cholesterol nanoparticles has been recently developed to measure RCT in humans. Similar to the in vivo assay using ${ }^{13} \mathrm{C} 2$-cholesterol, three main steps of the RCT can be estimated. However, in contrast to the ${ }^{13} \mathrm{C} 2$-cholesterol, the assay using ${ }^{3} \mathrm{H}$ - 
cholesterol nanoparticles allows to measure macrophage-specific cholesterol efflux. This method has been tested and validated in mice, and results of a study with healthy volunteers suggest that this method is feasible and safe for human applications [27].

Several human cell types have been used as donor cells, such as human monocytes and fibroblasts, THP-1 human monocyte-macrophages, HeLa cells, human umbilical vein endothelial cells (HUVEC), HepG2 human hepatocarcinoma cells, and platelets. Furthermore, animal-derived cells, such as RAW 264.7 or J774 murine macrophages, mouse fibroblasts, rat hepatoma (Fu5AH), Chinese hamster ovary-K1 cells (CHO-K1) and baby hamster kidney-21 (BHK) cells, have been used [28]. Cholesterol-efflux values from RAW 264.7 and J774 are highly correlated [29]. Donor cells can also be loaded with acetylated LDL, which results in the formation of a foam cell phenotype [28]. The most important pathways in the cholesterol efflux from macrophages are ABCA1/ABCG1-dependent. Indeed, macrophages lacking $A B C A 1$ and/or ABCG1 have a reduced cholesterol efflux capacity, whereas efflux capacity of macrophages lacking SR-BI was unchanged [30]. The expression of both ABCA1 and ABCG1 can be increased with an LXR agonist, such as TO-901317 [31]. Upregulation of theses transporters promoted macrophage cholesterol efflux in vitro, and increased macrophage RCT and reduced atherosclerosis development in mice [9, 32]. Murine cell lines can also be treated with cyclic adenosine monophosphate (cAMP) to upregulate $\mathrm{ABCA} 1$ expression, resulting in an increased cholesterol efflux to both apoA-I and HDL [6, 33, 34] (Figure 3).

Depending on the pathway of interest, several acceptors can be used. HDL and apoA-I are used to measure cholesterol efflux from macrophages via ABCA1, ABCG1 and SR-BI-dependent pathways. Cyclodextrin, an acceptor of cell surface free cholesterol, is used for non-specific cholesterol efflux. Cyclodextrins contain a hydrophobic cavity, in which cholesterol can be encapsulated to form soluble complexes $[28,35,36]$. However, the kinetics of cholesterol efflux to cyclodextrins is different from those of physiological cholesterol acceptors such as HDL. The amount of cholesterol released by the cells when cyclodextrin is used, is more rapid and the equilibrium between cells and medium is reached faster, which might be attributed to the higher molar ratio of cholesterol to cyclodextrin molecules $[35,36]$. 


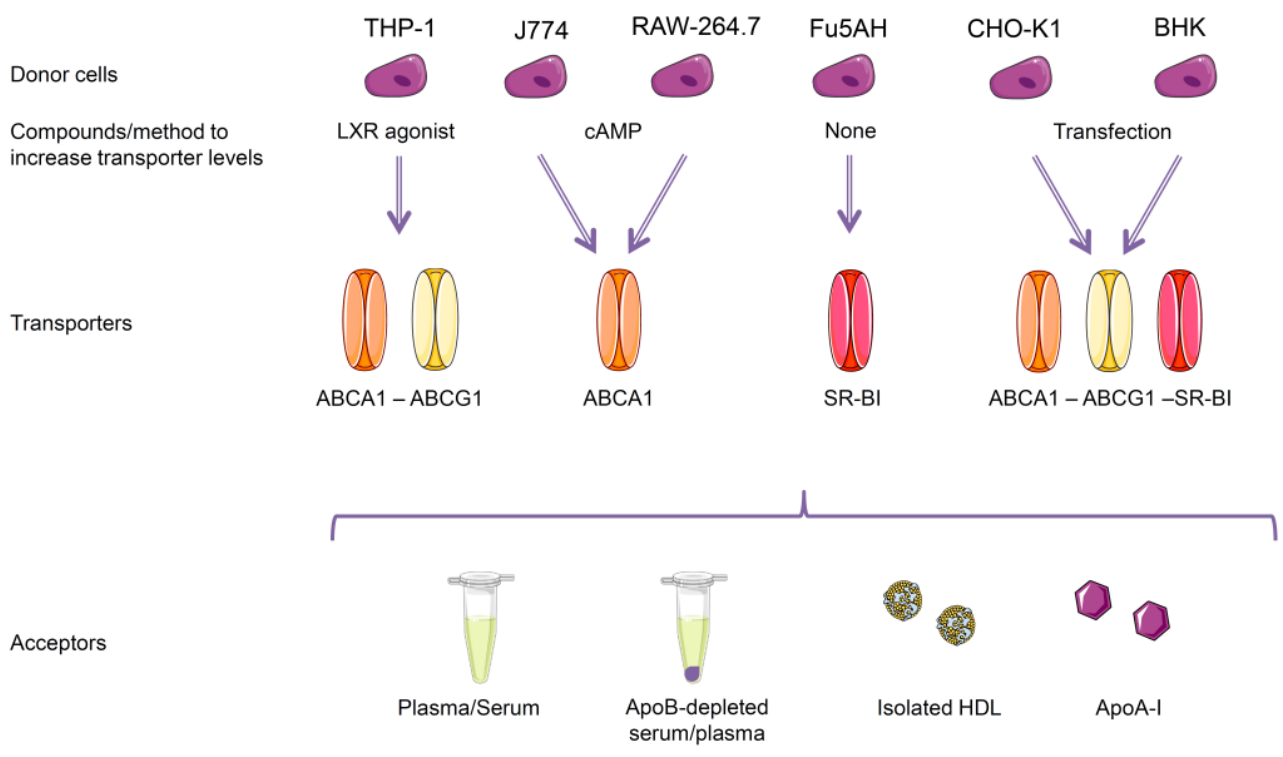

Figure 3: Commonly used donor cells, transporters and acceptors in cholesterol efflux assays. Figure was produced using Servier Medical Art (http://smart.servier.com/).

LXR: liver X receptor; cAMP: cyclic adenosine monophosphate; ABCA1: ATP-binding cassette 1; ABCG1: ATPbinding cassette sub-family G member 1; SR-BI: Scavenger receptor class B member I; apoB: apolipoprotein B; HDL: High-density lipoproteins; apoA-I: apolipoprotein A-I.

A frequently used assay to measure cholesterol efflux capacity of HDL particles has been described by Khera et al. [6]. In this assay, J774 murine macrophages are first cultivated and treated with an acyl-coenzyme A:cholesterol acyltransferase (ACAT) inhibitor to minimize cholesterol esterification to make sure that all labeled cholesterol will be present as free cholesterol. Macrophages are then labeled with ${ }^{3} \mathrm{H}$-cholesterol and incubated with cAMP for 24 hours to upregulate the expression of ABCA1. Next, human serum is depleted from apoB100-containing lipoproteins using polyethylene glycol (PEG), and the supernatant - containing the HDL particles - is added to the radiolabeled macrophages for 4 hours. Finally, the percentage of efflux of radioactive cholesterol from the cells to the acceptor as compared to a control pool serum is determined by liquid scintillation counting (Figure 4). This assay quantifies the efflux of cholesterol from macrophages through the ABCA1mediated pathway, which is activated by cAMP treatment. However, ABCG1- and SR-BI-dependent pathways, and aqueous diffusion of free cholesterol, also contribute to the measured cholesterol efflux. The individual contribution of each 
efflux-pathways has been previously investigated, using mouse peritoneal macrophages. ABCA1-mediated efflux has been demonstrated to play a major role. However, aqueous diffusion has also been shown to be one of the major contributors of cholesterol efflux, accounting for 35\%, and ABCG1 accounted for approximately $20 \%$ of the efflux. However, the contribution of SR-BI to efflux was small, about $9 \%$ [37]. As all these transporters are part of the murine J774 macrophages, their activities are comparable within one analytical run. However, as the HDL fraction added to the radiolabeled macrophages is subject-specific, the capacity of a person's HDL to promote cholesterol efflux is measured. Furthermore, other transporters, such as ABCA8, can be involved in cholesterol efflux [38]. It should be noted that this assay focuses on the interaction between the HDL fraction with the ABCA1 transporter, and not on the effects of the intervention on ABCA1 expression. Any changes in cholesterol efflux capacity are therefore not due to changes in ABCA1 expression, but to differences in the composition of the supernatant used, including HDL particle size or composition. When using apoB100-depleted serum, other mediators of cholesterol efflux might be present in the supernatant, including proteins such as albumin [39] and plasminogen [40], and exosomes [41], which can also contribute to the ABCA1-mediated cholesterol efflux. Moreover, only a part of the RCT pathway is determined, as uptake and metabolism of cholesterol in the liver and TICE is not quantified. Furthermore, results do not depend on variations in the rate of hydrolysis of $\mathrm{CE}$, the status of endogenous macrophage cholesterol transporters, and the transfer of CE from HDL to apoB100-containing lipoproteins via CETP [6]. Moreover, the method to isolate HDL can affect HDL particles size and/or composition. When PEG is used for serum apoB100 depletion, cholesterol efflux capacity was shifted towards smaller HDL particles, although the total efflux summed across all HDL-sized fractions did not differ between the control serum sample and apoB-depleted serum samples. The phospholipid and cholesterol profile of the HDL size fractions was also shifted to the right, indicating a change to smaller sized particles. Three other apoB precipitation methods (dextran sulfate/magnesium chloride, heparin sodium/manganese chloride, and LipoSep immunoprecipitation) also changed the quantity of some apolipoproteins, such as a reduction in apoE concentrations without affecting the size distribution of HDL particles subfractions. None of these three methods, however, affected the efflux of cholesterol [42]. 


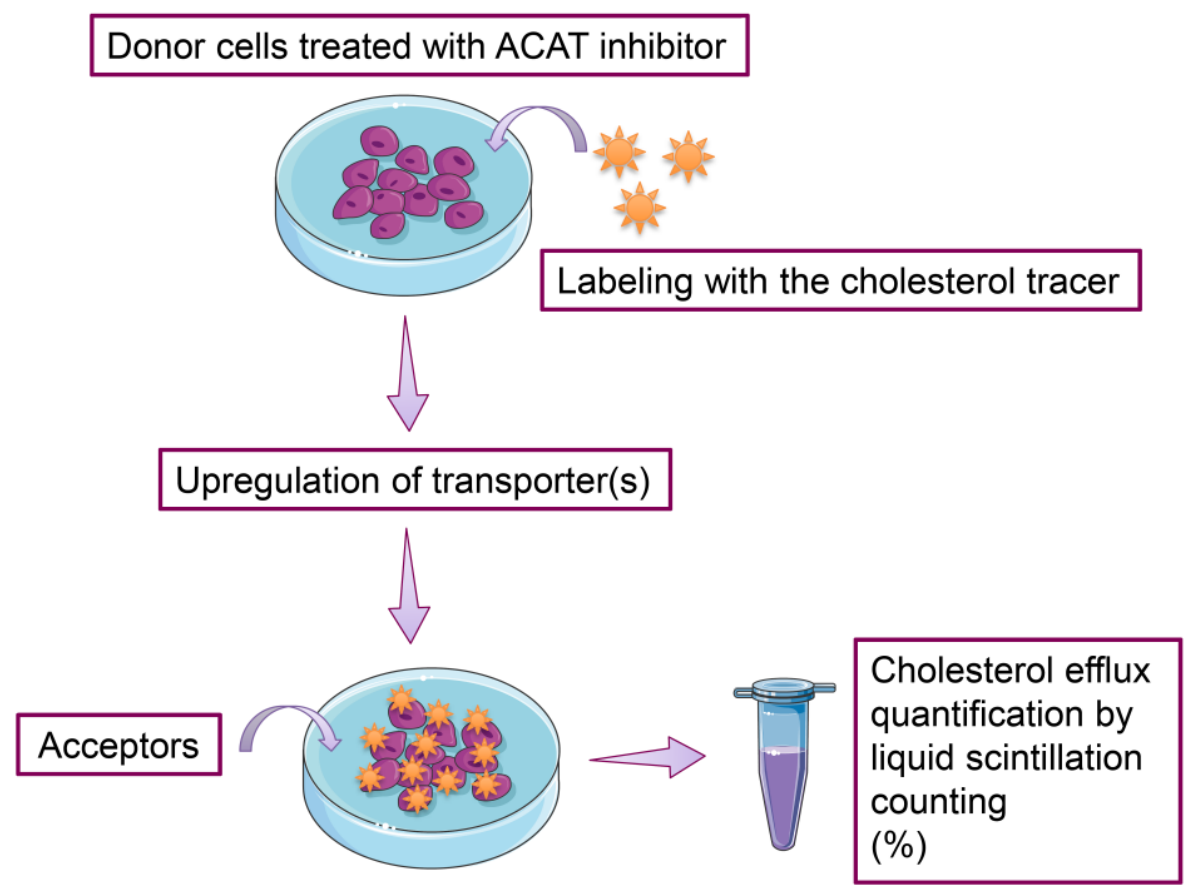

Figure 4: Cholesterol efflux assay. Figure was produced using Servier Medical Art (http://smart.servier.com/).

\section{Determinants of cholesterol efflux}

\section{HDL-C concentrations}

Cholesterol efflux capacity correlates positively with HDL-C and apoA-I concentrations [6, 7]. However, this does not necessarily mean that increasing HDLC or apoA-I concentrations increases cholesterol efflux capacity [43]. CETP inhibitors, for example, effectively raise HDL-C and apoA-I concentrations, but not all CETP inhibitors affects cardiovascular outcomes. Indeed, no beneficial effects of torcetrapib, dalcetrapib and evacetrapib on cardiovascular events were found, while the impact of TA-8995 is not yet known [44]. To date, only anacetrapib resulted in a significantly lower incidence of major coronary events [45]. 


\section{HDL particles size and composition}

HDL consists of many different particles, which are heterogeneous in structure, density, size, composition, and biological activity [11]. Mature HDL particles consist of a surface monolayer rich in phospholipids and proteins, and a hydrophobic core made of CE and TG, whereas pre $\beta-1$ HDL particles mainly consist of apoA-I and a small amount of phospholipids and free cholesterol [46]. Small and dense pre $\beta-1$ HDL particles promote cholesterol efflux primarily through the ABCA1 transporter, whereas large and less dense HDL particles mainly through the ABCG1 and SR-BI transporters [11]. However, there is no unique nomenclature for HDL subfractions, which relate to the separation technique used. With ultracentrifugation, two main subfractions of HDL can be separated, i.e. the large HDL2 and the smaller HDL3 particles, which can be further differentiated into HDL2b, HDL2a, HDLзa, HDL3b and HDL3c particles. With 2D electrophoresis, even more than ten HDL subfractions can be identified [11, 47]. Other methods to isolate HDL subfractions include fast protein liquid chromatography, immune electrophoresis, and nuclear magnetic resonance [20]. Due to these different techniques, relationships between HDL subfractions with CVD risk might be difficult to compare between studies [48].

Phosphatidylcholine is the predominant phospholipid in HDL (74-80\%), followed by sphingomyelin (14-20\%), lysophosphatidylcholine (1.5-3.3\%), phosphatidylinositol (1.6-2.0\%), phosphatidylethanolamine $(1.4-2.1 \%)$, ceramide (0.11-0.19\%), phosphatidylserine (0.03-0.63\%), phosphatidylglycerol (0.011-0.015\%), and finally phosphatidic acid $(0.010-0.025 \%)[46,49]$. As compared to HDL3, the surface monolayer of $\mathrm{HDL}_{2}$ particles is rich in sphingomyelin and ceramide. In contrast, small $\mathrm{HDL}_{3}$ particles are preferentially associated with phosphatidylcholine, lysophosphatidylcholine, phosphatidylserine and phosphatidic acid [49]. The mechanisms responsible for the heterogeneity in phospholipids composition between HDL subpopulations are unclear, but may be due to the varying amounts of phospholipids that can be sequestered in the boundary layer, by interaction with apoA-I at the disc edge [50]. The phospholipid composition of HDL particles may be a major determinant of cholesterol efflux, as it determines surface lipid fluidity [46]. A high content in sphingomyelin (expressed as the percentage of the total HDL mass) found in HDL2 particles, is associated with a decreased cholesterol efflux, due to a decrease in the HDL surface fluidity [51]. In contrast, the content of phosphatidylserine in HDL3 particles is positively associated with cholesterol efflux capacity [46, 49,52], supporting studies emphasizing that small HDL3 particles promote cholesterol efflux [53]. Also, an abnormal HDL lipid composition, such as enrichment in TG and reduction in phospholipids, impairs the ability of HDL to promote cholesterol efflux [46]. In fact, CVD risk has been associated with decreased concentrations of total HDL phospholipids, independent 
of HDL-C concentrations and particle sizes [54]. The association between HDL particles size and composition with CVD has been investigated in several studies. As reviewed, the number of large HDL2 particles is negatively correlated with CVD risk. Moreover, a reduction in HDL particle size is positively associated with CVD [55], and small HDL3 particles are associated with an increased risk of developing CVD in healthy men and women [56]. These epidemiological findings seem to contradict findings from efflux studies, suggesting that both pre $\beta$-1 HDL [57] and the smaller HDL3 particles [53] are highly efficient to promote cholesterol efflux via the ABCA1 transporter.

\section{HDL-C and apoA-I raising drugs}

CETP-inhibitors may have differential effects on cholesterol efflux. Dalcetrapib increased non-ABCA1-mediated cholesterol efflux, but not ABCA1-dependent cholesterol efflux. However, cholesterol efflux capacity only increased by one-tenth, as compared to the one-third increase in HDL-C concentrations [58]. In contrast, ABCA1- and ABCG1-mediated cholesterol efflux was increased in subjects treated with anacetrapib [59]. In addition, the CETP inhibitors evacetrapib [60, 61] and TA8995 [62] increased cellular cholesterol efflux capacity via ABCA1 and non-ABCA1 mechanisms. The increase in cholesterol efflux capacity by evacetrapib and TA-8995 correlated positively with the increase in pre $\beta-1$ HDL particles concentrations, independently of the increase in HDL-C concentrations. The increase in HDL2 and decrease in $\mathrm{HDL}_{3}$ concentrations were not associated with cholesterol efflux. Surprisingly, a common SNP $(-629 \mathrm{C} \rightarrow \mathrm{A})$ in the CETP gene, which translates into higher plasma CETP concentrations and lower HDL-C concentrations, was associated with a greater cholesterol efflux capacity [63].

RVX-208 increases HDL-C and apoA-I concentrations in vitro and in vivo via interaction with bromodomains [64]. In African green monkeys, RVX-208 increased concentrations of pre $\beta-1 \mathrm{HDL}, \mathrm{HDL}_{2}$ and $\mathrm{HDL}_{3}$, as well as cholesterol efflux capacity via ABCA1, ABCG1, and SR-BI pathways. In humans, oral treatment for 1 week increased plasma concentrations of apoA-I and pre $\beta-1 \mathrm{HDL}$, and cholesterol efflux capacity via the ABCA1-mediated pathway [65].

ApoA-I-mimetic peptides promoted the formation of pre $\beta-1$ HDL and stimulated cholesterol efflux from macrophages via ABCA1. This increase in cholesterol efflux correlated positively with the increase in pre $\beta-1$ HDL. However, the safety of these apoA-I-mimetic peptides needs further investigations, as administration at high doses in mice lead to an accumulation of VLDL-TG $[66,67]$. 


\section{Lipid-lowering drugs}

Statins effectively lower LDL-C and thereby CVD risk by inhibiting 3-hydroxy-3methylglutaryl coenzyme A (HMG-CoA) reductase, the rate-limiting enzyme in cholesterol synthesis [68]. Statins have pleiotropic effects and many statins (e.g. rosuvastatin, atorvastatin, simvastatin, and pravastatin) modestly elevate HDL-C concentrations. Results for changing apoA-I concentrations were less pronounced $[69,70]$. Further, atorvastatin and rosuvastatin significantly increased the concentration of the large HDL2, and decreased small $\mathrm{HDL}_{3}$ and pre $\beta-1 \mathrm{HDL}$ concentrations [71, 72]. In addition, simvastatin increased the total phospholipid concentration of HDL by increasing the content of phosphatidylcholine and phosphatidylethanolamine [73]. Although particle size distribution and HDL composition are important determinants of cholesterol efflux, effects of statins on cholesterol efflux are less clear. In mice, rosuvastatin did not change HDL-C concentrations, but increased the capacity of HDL to promote cholesterol efflux via the ABCA1 pathway [74]. Other studies in mice [74] or humans [6, 75, 76], however, found no effects of atorvastatin [6,74,76], as well as of pravastatin [6] and simvastatin [75] on cholesterol efflux capacity. However, treatment of human macrophages with simvastatin and atorvastatin decreased the ABCG1-mediated cholesterol efflux pathway without affecting ABCG1 protein levels [77]. Moreover, incubation of human macrophages with atorvastatin or simvastatin increased miR33 expression, together with a decrease in ABCA1 mRNA expressions, and simvastatin decreased ABCA1-mediated cholesterol efflux [78]. Incubation of J774 mouse macrophages with several types of statins, i.e. simvastatin, pitavastatin, atorvastatin and rosuvastatin, reduced ABCA1 mRNA expression as well as the ABCA1-mediated cholesterol efflux from macrophages, with simvastatin having the most pronounced effects [78]. Thus, if statins have beneficial effects on cholesterol efflux capacity is still unknown, and the different statins may differently impact cholesterol efflux capacity of HDL particles.

Niacin increased concentrations of HDL-C and HDL2 particles, but had only a minor effect on serum cholesterol efflux capacity [79]. ABCG1- and ABCA1-mediated cholesterol efflux did not change, while only small increases or no changes at all via SRB1-mediated efflux have been reported. These small changes in cholesterol efflux were positively associated with the changes in HDL-C concentrations [80]. Also, combination therapy of niacin with statins increased ABCA1-mediated cholesterol efflux from macrophages [76].

Fibrates raise HDL-C concentrations in patients with atherogenic dyslipidemia [81], and bezafibrate increased cholesterol efflux capacity in type 2 diabetes patients [82]. In another study, fenofibrate slightly increased cholesterol efflux via the SRB1mediated pathway, but did not affect the ABCA1-mediated pathway. This latter 
effect may be explained by the lack of effect on concentrations of small pre $\beta-1$ HDL, the preferred acceptor of cholesterol via the ABCA1-mediated pathway [79].

\section{Gender}

It is well known that HDL-C concentrations are higher in pre-menopausal women than in men [83]. HDL from pre-menopausal women also has a greater cholesterol efflux capacity as compared to HDL from men, independently of differences in HDL-C concentrations [84]. It has also been reported that women have more HDL2 particles than men [85], and these larger HDL2 particles were related to an increased cholesterol efflux capacity via the SR-BI pathway in healthy women with increased HDL-C concentrations. However, it has also been observed that men have more pre $\beta-1$ HDL particles, leading to an increased efflux capacity via the ABCA1 pathway, although total cholesterol efflux capacity remained higher in women [86]. After the menopause, HDL-C and HDL2 particle concentrations are decreased, whereas the concentration of HDL3 particles is increased [87-89]. However, effects of menopause on cholesterol efflux capacity are not well understood. Corcoran et al. observed that estrogen reduced the CE content in human monocyte-derived macrophages via the activation of the estrogen receptor on the macrophage, without any effect on cholesterol efflux capacity [90]. Badeau et al. also found no relation between cholesterol efflux capacity with gender, menopausal status, or estradiol concentrations, when using samples from a large-scale population-based study [91]. However, the same authors reported earlier that cholesterol efflux capacity of HDL particles in women might be linked to the estradiol esters incorporated in HDL particles [92]. When HDL from 8 men was incubated with estradiol oleate, cholesterol efflux from macrophages increased significantly. Since SR-BI participates in the uptake of estradiol in hepatocytes [92], the involvement of this receptor on the macrophage on cholesterol efflux was studied. Treatment of macrophages with an SR-BI inhibitor or an estrogen receptor antagonist reduced cholesterol efflux capacity mediated by estradiol oleate enriched HDL. This indicated that both estrogen receptors and SR-BI are involved in the increased cholesterol efflux from macrophages stimulated by HDL-associated estradiol oleate [84]. In a longitudinal study in which women were studied before and after menopause, cholesterol efflux capacity correlated positively with both medium and large HDL particles concentrations, before and after menopause, as quantified by ion mobility analysis (IMA). The number of large HDL particles increased after menopause, whereas the size of small HDL particles decreased [93]. The authors speculated that these findings might be explained by the fact that the subjects of the study were women in an early stage of the menopause, i.e. the time between the last menstrual period and the follow-up measurements was only 2 years on average. To summarize, pre- 
menopausal women have a higher cholesterol efflux capacity than men, but studies examining changes in cholesterol efflux during and after the menopause are not unequivocal.

\section{BMI}

An increased BMI is associated with a decrease in apoA-I and HDL-C concentrations. Moreover, obese subjects have lower HDL2 and higher HDLs particle concentrations [94-98]. Further, pre $\beta-1$ HDL particle concentrations may be higher in obese subjects as compared to lean subjects, which has been explained by increased hepatic lipase and phospholipid transfer protein (PLTP) activities in obese subjects $[98,99]$. Obesity is also associated with a decreased expression of genes involved in cholesterol metabolism, such as ABCA1 and ABCG1. This may explain why obese people have a reduced cholesterol efflux capacity [100], despite increased pre $\beta-1$ HDL particle concentrations. In addition, cross-sectional differences in efflux capacity between various BMI-groups have previously been reported [101-103]. Weight reduction is accompanied by increases in HDL2 concentrations and decreases in those of HDL3 in both men and women [104], as explained by the decreases in hepatic lipase and CETP activities [105]. The increase in plasma concentrations of HDL2 after weight loss induced by bariatric surgery is associated with an improvement in cholesterol efflux capacity via the SR-BI and ABCG1 pathways [106]. In fact, efflux via the ABCA1 pathway was decreased, most likely due to reduced pre $\beta-1$ HDL concentrations [105]. Two others studies have shown that weight loss induced after surgery resulted in an increase in HDL-cholesterol efflux capacity after upregulation of ABCA1 expression [107, 108], and in large HDL2 concentrations [107]. However, the type of intervention used to induce weight loss, such as bariatric surgery or calorie restriction, may lead to different findings. Indeed, weight loss by very low calorie diet was found to increase apoA-I concentrations, without improving cholesterol efflux [109]. In two other studies, diet-induced weight loss also failed to improve cholesterol efflux [110, 111]. As already discussed, the sphingomyelin content of HDL particles correlates negatively with cholesterol efflux, and weight loss induced by an energy-restricted diet is associated with a decrease in the sphingomyelin content of all HDL subclasses [112], which might explain the improvement of efflux capacity after weight loss. Thus, surgery-induced weight loss may increase cholesterol efflux, but studies on diet-induced weight loss are less clear. Whether this relates to the amount of weight loss, which is higher after surgery, warrants further investigation. 


\section{Age}

Aging is associated with an increased risk of developing CVD and a decrease in HDL-C concentrations [113, 114]. Age also correlates positively with HDL2 particle concentrations and negatively with those of HDL3 [115]. Cholesterol efflux was lower in elderly healthy subjects as compared to young healthy subjects, mainly via a reduced efflux via the ABCA1 pathway. Moreover, cholesterol efflux capacity of $\mathrm{HDL}_{3}$ particles significantly decreased with aging, whereas no difference was found for HDL2 particles. In elderly subjects, cholesterol efflux was positively associated with large HDL particles concentrations and inversely with those of small HDL particles [116]. Oxidative processes have been shown to impair HDL-mediated cholesterol efflux capacity in vitro [117]. As in vitro studies suggest that aging is associated with an increased susceptibility of HDL to oxidize, this might at least partly explain decrease in the cholesterol efflux observed in elderly [118, 119]. Another possible explanation relate to a reduction in elderly subjects of the phosphatidylcholine/sphingomyelin ratio and the fluidity of the phospholipid bilayer of HDL particles [118]. Also, gene and protein expressions of ABCA1 and ABCG1, as well as cholesterol efflux capacity, were reduced in macrophages isolated from elderly mice and humans [120]. Moreover, experiments in knockout mice showed that cholesterol efflux was similar when macrophages of young mice depleted of ABCA1, but not of ABCG1 - were compared with macrophages of old mice. It was suggested that the decrease in ABCA1 expression in macrophages from elderly mice might be due to the upregulation of miR-33 expression in macrophages [120], which may be involved in the downregulation of ABCA1 and ABCG1 expression [121]. Another study found reduced apoE levels, an apolipoprotein carried by HDL which may promote ABCA1-mediated cholesterol efflux [122], and increased sphingomyelin content in the total HDL fraction of healthy elderly subjects, as compared to young subjects. However, cholesterol efflux capacity of HDL particles was not different [123]. Still, most of the studies conclude that aging is associated with a decrease in cholesterol efflux capacity.

\section{Lifestyle}

Changing lifestyle, such as increasing physical activity, quit smoking or adopting a healthy diet, effectively improves health. Physical activity increased ABCA1 expression in human leukocytes, as well as plasma concentrations of apoA-I and pre $\beta-1$ HDL. In addition, ABCA1 expression correlated positively with pre $\beta 1-\mathrm{HDL}$ concentrations [124]. Endurance-trained athletes have an increased cholesterol efflux capacity, which is in line with higher concentrations of apoA-I, HDL-C and pre $\beta-1$ HDL as compared to control subjects [125]. Endurance training also increased 
the proportion of large HDL2 particles [96]. As reviewed, smoking reduces HDL-C concentrations, which has been explained by a reduction in the larger HDL2 particles $[126,127]$. Furthermore, ABCA1 protein expression was lower in macrophages from smokers as compared with nonsmokers, while ABCA1-mediated cholesterol efflux capacity was also reduced in smokers. Three months after smoking cessation, ABCA1 protein and mRNA expressions, as well as ABCA1-mediated cholesterol efflux were increased, while no changes on ABCG1 expression and function were found. However, for unknown reasons, this was only evident in patients with coronary artery disease (CAD), but not CAD subjects without CAD [128].

Diet also affects cholesterol efflux capacity. The amount of alcohol consumed is positively associated with ABCA1 expression and cholesterol efflux capacity [124]. Consumption of 4 glasses of whisky daily for 17 days increased ABCA1-mediated serum cholesterol efflux from J774 mouse macrophages, as well as pre $\beta-1$ HDL concentration [129]. Consumption of a fatty meal by normolipidemic subjects increased the capacity of whole plasma to stimulate cholesterol efflux during the postprandial phase, concomitant with an increase in CETP activity. For both parameters, the maximum was reached after eight hours. Moreover, HDL-C concentrations slightly decreased, whereas HDL-phospholipids increased. No changes were observed in apoA-I concentrations during the postprandial phase. In addition, plasma cholesterol efflux capacity positively correlated with the concentrations of HDL-C, apoA-I and total HDL-phospholipids during the postprandial phase [130]. Acute consumption of walnuts improved postprandial cholesterol efflux from J774 macrophages, as compared to the fasting condition. However, this effect was only found when whole serum was used, but not apoBdepleted serum. The authors therefore suggested that walnuts might have a greater impact on global cholesterol efflux rather than on efflux mediated by only HDL particles, and that LDL particles could as well play a role in the RCT pathway as an acceptor of free cholesterol [131]. In mice, dietary supplementation with $\beta$-carotene increased cholesterol efflux from macrophages and the expression of ABCA1 and ABCG1 [132]. Effects of fatty acids rich diets have already been reviewed and are controversial [126, 133]. Macrophage cholesterol efflux was not changed by diets rich in trans, polyunsaturated, or saturated fatty acids [134], despite a significant decrease in HDL-C concentrations [135]. In vitro, cholesterol efflux from macrophages was decreased after treatment with various unsaturated fatty acids, i.e. palmitoleate, oleate, linoleate, and arachidonate, by enhancing the degradation of ABCA1. In contrast, no effects of the saturated fatty acids octanoate, palmitate, and stearate were found [136]. Beneficial effects of dietary fatty acids have also been reported. Hamsters fed an omega 3 rich diet (rich in EPA and DHA) improved cholesterol efflux capacity [137]. Similar results have been found in humans who received a supplement with EPA, twice a day for 4 weeks [138]. Moreover, 
conjugated linoleic acid isomers, i.e. c9t11-CLA and t10c12-CLA, increased in vitro cholesterol efflux from macrophages by up-regulating ABCA1 expression [139]. Consumption of extra-virgin olive oil, rich in MUFA, increased ABCA1 and ABCG1 expression in macrophages, as well as cholesterol efflux capacity of HDL [140], and polyphenol-rich olive oil increased the expression of key genes involved in cholesterol efflux, such as ABCA1 or SR-BI [141]. Cholesterol efflux was also enhanced after consumption of a polyphenol-rich olive oil. This oil also induced changes in the HDL particles size distribution. Large HDL2 concentrations increased, while small HDLs particles decreased, which may explain the enhancement in the efflux capacity observed in this study [142], suggesting that the improvement in efflux capacity might be modulated by the ABCG1-mediated pathway. A recent study from the same group showed that 1 year of intervention with two types of Mediterranean diets, i.e. one enriched with virgin olive oil and one enriched with nuts, both increased cholesterol efflux capacity and large $\mathrm{HDL}_{2}$ particles concentrations. Moreover, the consumption of the virgin olive oil enriched diet decreased CETP activity and increased the total phospholipids content in the HDL surface [143]. It has been suggested that consumption of extra-virgin olive oil increased the fluidity of the phospholipidic layer of HDL [140].

\section{Genetic background}

Villard et al. showed that cholesterol efflux capacity of HDL particles from macrophages was related to SNPs located in genes involved in HDL metabolism, such as CETP (rs17231506), ABCA1 (rs2230806), APOA1 (rs1799837), APOAII (rs5086), and LIPC (lipase C; rs1800588) [144]. Moreover, these associations were independent of HDL-C concentrations and gender-specific. However, the seven SNPs presented in this study explained only $6 \%$ of the plasma cholesterol efflux variability. In another study, it was found that SNPs located in STAB1 (stabilin-1; rs13326165), SLC39A8 (solute carrier family 39 member 8; rs13107325), CITED2 (cbp/p300-interacting transactivator 2; rs605066), CETP (rs3764261), ANGPTL4 (angiopoietin like 4; rs7255436). SNPs located in LIPC (rs1532085) and ANGPTL8 (angiopoietin like 8; rs737337) were the most strongly associated with cholesterol efflux [145].

\section{Inflammation}

As recently reviewed, acute inflammation is associated with remodeling of the proteins carried by HDL particles, changing their capacity to mediate cholesterol efflux and thereby RCT [146]. Acute phase responses to inflammation decrease 
HDL-C concentrations, hepatic apoA-I production, and ABCA1 and ABCG1 expressions in THP-1 macrophages [147]. Also, HDL particles become enriched in serum amyloid A (SAA) [148], leading to the formation of larger and dysfunctional HDL particles. Levels of SAA in endotoxin-treated mice and men were negatively related with efflux capacity [149]. Inflammation induced with lipopolysaccharide may also block the final step of the RCT, i.e. the transport of cholesterol through the liver into the bile, by down-regulating the expression of specific biliary transporters (ABCG5 and ABCG8), as observed in mice [150]. In contrast, the acute phase response slightly enhanced ABCG1-mediated efflux in mice. This may be due to the enrichment in the phospholipids content of HDL after acute inflammation, which is not observed in humans [151].

Besides the extreme condition of an acute phase response, a more mild condition like low-grade systemic inflammation may also affect cholesterol efflux. This condition is characterized by increased concentrations of many pro-inflammatory cytokines, such as tumor necrosis factor- $\alpha$ (TNF- $\alpha$ ) and interleukin-6 (IL-6), and is often found in obese subjects [152]. Treatment of THP-1 macrophages with TNF- $\alpha$ decreases ABCA1 mRNA and protein expressions, as well as HDL-mediated cholesterol efflux, while no effect of TNF- $\alpha$ was found on ABCG1 mRNA and protein expressions [153]. Moreover, weight loss decreases low-grade systemic inflammation [154], which may contribute to the increase in cholesterol efflux capacity after loss of fat mass.

\section{Conclusion}

This review focused on the determinants of cholesterol efflux capacity, an important determinant of the RCT pathway. Results of the determinants discussed here have been summarized in the Table 1. Recent studies have suggested that increasing HDL functionality is more important than increasing HDL-C or apoA-I concentrations to lower CVD risk $[6,7,155,156]$. Indeed, increasing HDL-C or apoA-I concentrations do not necessarily improve cholesterol efflux capacity. For instance, CETP inhibitors do not consistently increase cholesterol efflux capacity, even though HDL-C concentrations are increased. The ABCA1 transporter is the major route for cholesterol efflux from macrophages and increasing tissue specific mRNA and/or protein expressions of this transporter may be an important target to decrease CVD risk. Several efflux assays focusing on this pathway have therefore been developed, which have shown that the size and composition of HDL particles, and the different HDL subfractions, differently affect cholesterol efflux. However, these assays do not measure the effects of the interventions on $\mathrm{ABCA} 1$ expression, but principally the interaction between HDL particles and this transporter. Pre $\beta-1$ HDL particles are 
thought to be the most efficient to promote cholesterol efflux via the ABCA1 pathway, which is consistent with the findings of RVX-208 and apoA-I-mimetic peptides. ABCG1 and SR-BI are mainly involved in cholesterol efflux to HDL2 particles, which are considered to contribute less to HDL-mediated efflux [37]. Indeed, in elderly people, cholesterol efflux capacity is decreased, even though HDL2 particles concentrations are increased. In addition, interventions using niacin, trans and saturated fatty acids showed changes in HDL2-C concentrations, but not in efflux capacity. It is therefore surprising that prospective epidemiological cohort studies have observed that increased HDL2 particle concentrations are related to a reduced CVD risk. Therefore, it is essential to understand these apparent discrepancies. Except for apoA-I, HDL particles carry also other proteins, which determine efflux capacity as well. The intrinsic characteristics of the HDL particles are related to subjects' characteristics, and cholesterol efflux mediated pathways may for example be gender-specific, with the ABCA1-mediated pathway being more efficient in men than in women. In addition, lifestyle changes, such as lowering fat mass, increasing physical activity, quit smoking and eating a healthy diet, can modify HDL particles characteristics and distributions, and thereby cholesterol efflux capacity. However, more research is needed to determine the best targets to optimize in vivo cholesterol efflux capacity and whether these changes translate into a changed CVD risk. 


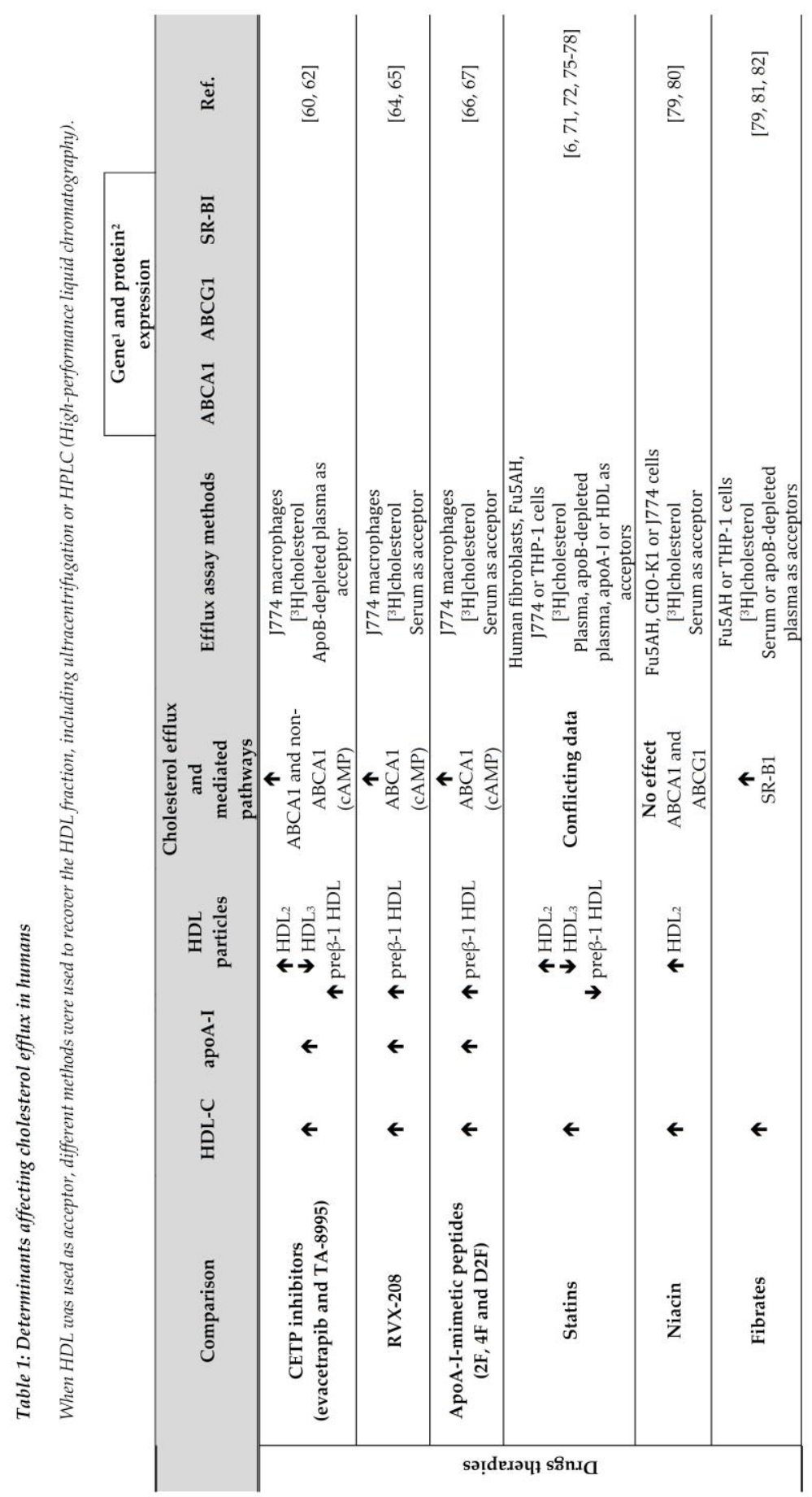




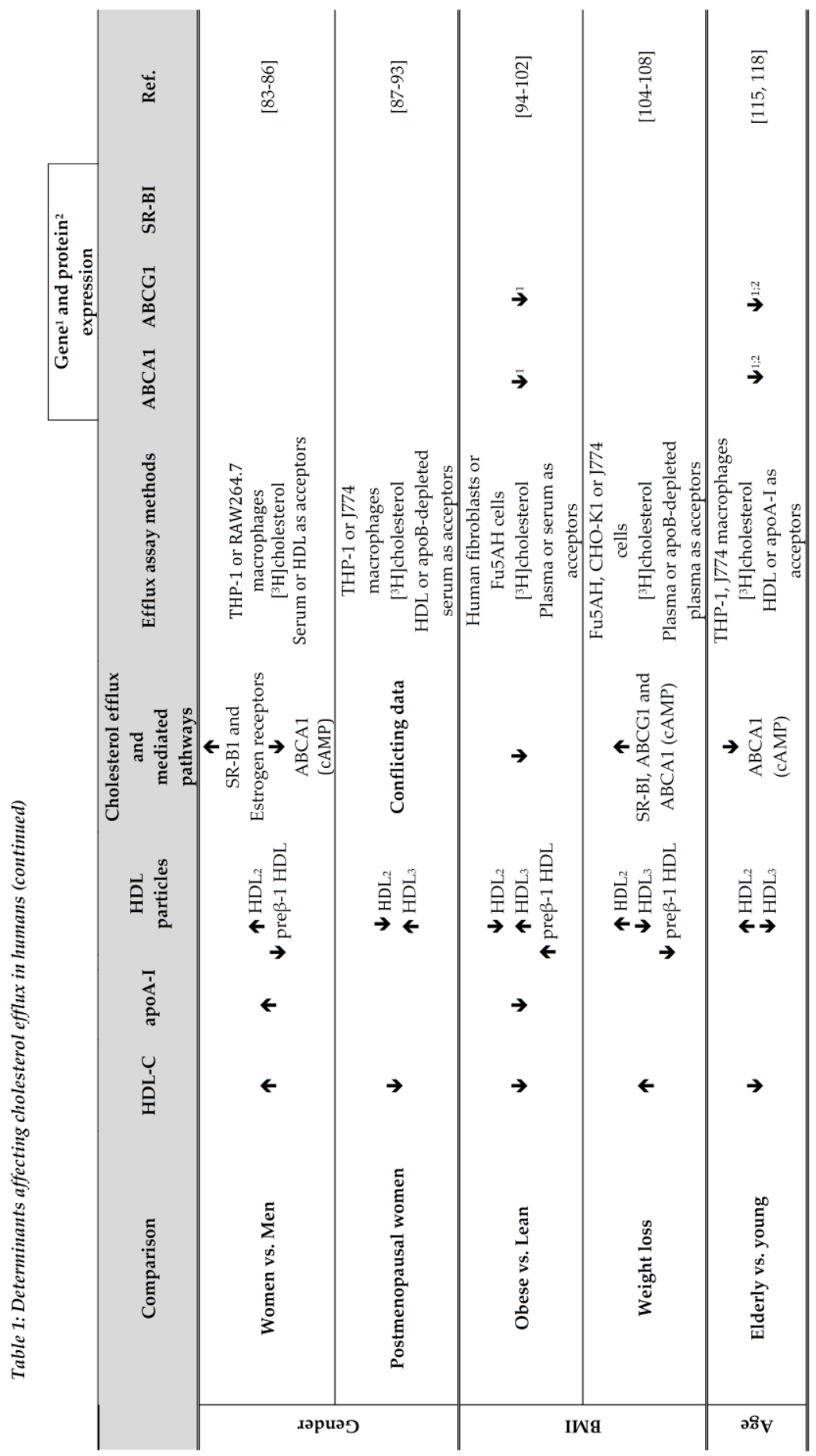




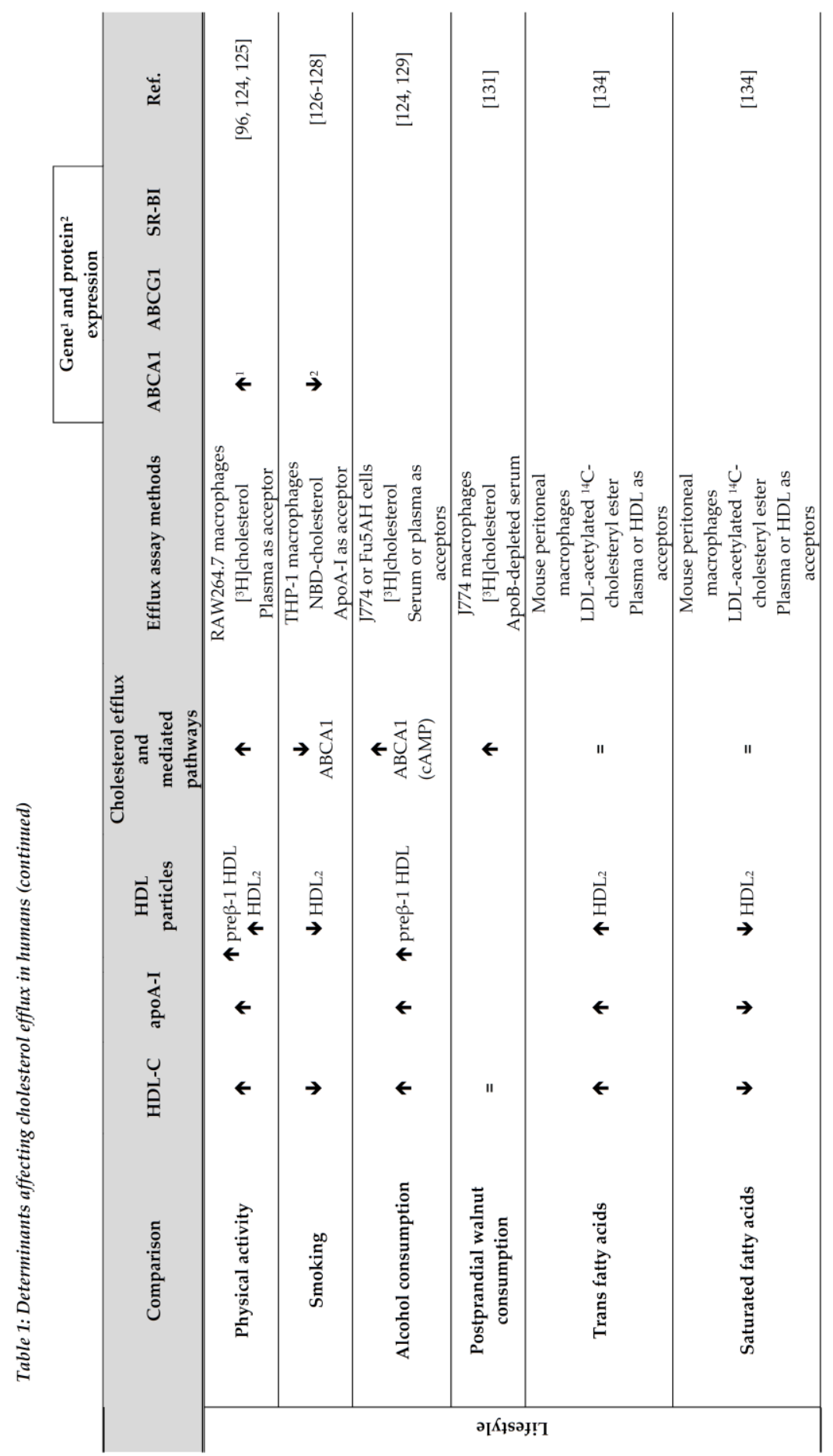




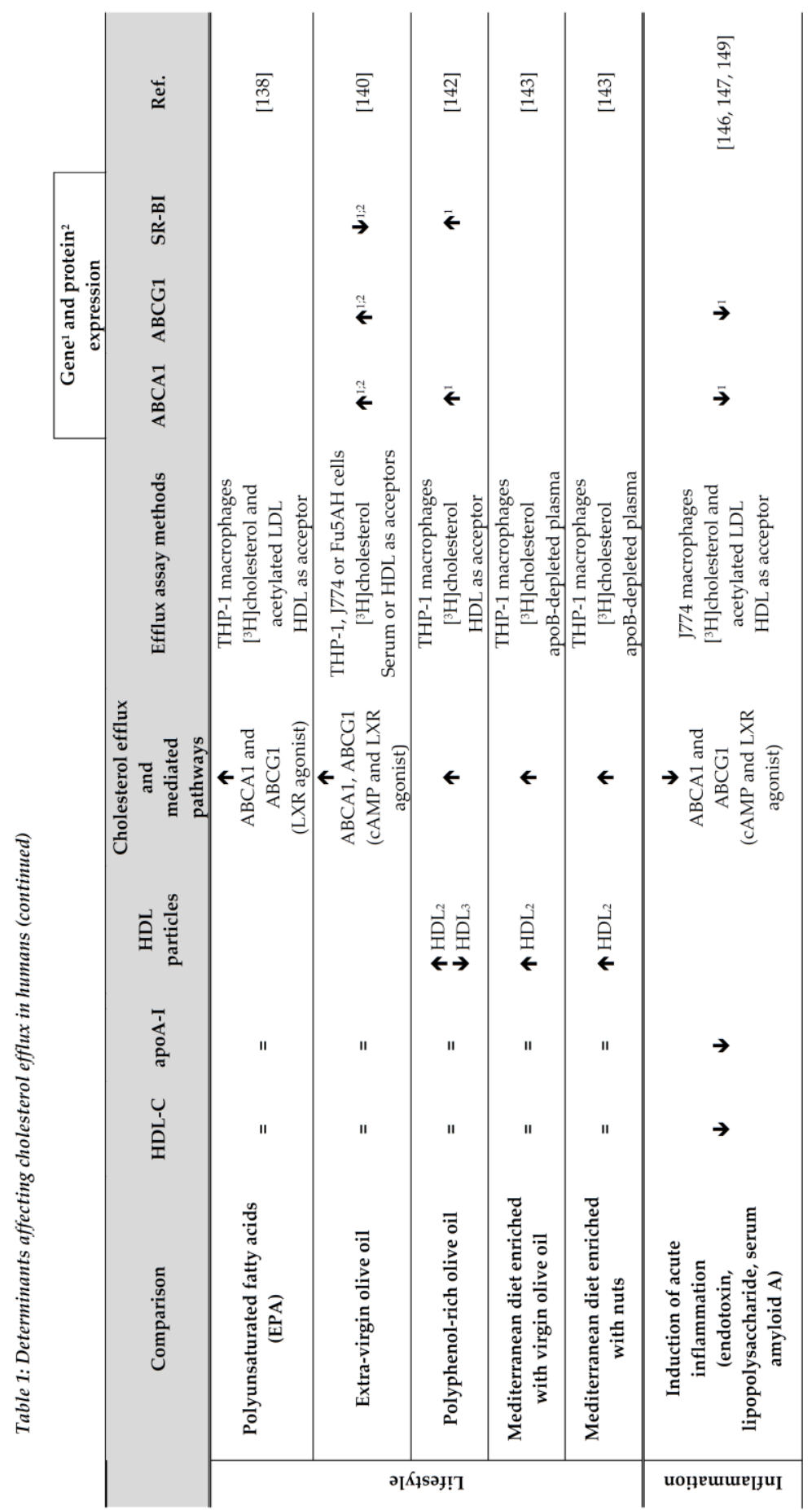




\section{References}

1. Wilson, P.W., R.D. Abbott, and W.P. Castelli, High density lipoprotein cholesterol and mortality. The Framingham Heart Study. Arteriosclerosis, 1988. 8(6): p. 737-41.

2. Barter, P., et al., High density lipoproteins (HDLs) and atherosclerosis; the unanswered questions. Atherosclerosis, 2003. 168(2): p. 195-211.

3. Choi, B.G., et al., The role of high-density lipoprotein cholesterol in the prevention and possible treatment of cardiovascular diseases. Curr Mol Med, 2006. 6(5): p. 571-87.

4. Rader, D.J. and G.K. Hovingh, HDL and cardiovascular disease. Lancet, 2014. 384(9943): p. 618-25.

5. Tall, A.R., Cholesterol efflux pathways and other potential mechanisms involved in the athero-protective effect of high density lipoproteins. J Intern Med, 2008. 263(3): p. 256-73.

6. Khera, A.V., et al., Cholesterol efflux capacity, high-density lipoprotein function, and atherosclerosis. N Engl J Med, 2011. 364(2): p. 127-35.

7. Saleheen, D., et al., Association of HDL cholesterol efflux capacity with incident coronary heart disease events: a prospective case-control study. Lancet Diabetes Endocrinol, 2015. 3(7): p. 507-13.

8. Ritsch, A., H. Scharnagl, and W. Marz, HDL cholesterol efflux capacity and cardiovascular events. N Engl J Med, 2015. 372(19): p. 1870-1.

9. Cuchel, M. and D.J. Rader, Macrophage reverse cholesterol transport: key to the regression of atherosclerosis? Circulation, 2006. 113(21): p. 2548-55.

10. Seo, H.S. and M.H. Choi, Cholesterol homeostasis in cardiovascular disease and recent advances in measuring cholesterol signatures. J Steroid Biochem Mol Biol, 2015. 153: p. 72-9.

11. Camont, L., M.J. Chapman, and A. Kontush, Biological activities of HDL subpopulations and their relevance to cardiovascular disease. Trends Mol Med, 2011. 17(10): p. 594-603.

12. Yamashita, S., et al., Molecular mechanisms of HDL-cholesterol elevation by statins and its effects on HDL functions. J Atheroscler Thromb, 2010. 17(5): p. 436-51.

13. Rader, D.J., et al., The role of reverse cholesterol transport in animals and humans and relationship to atherosclerosis. J Lipid Res, 2009. 50 Suppl: p. S189-94.

14. Rye, K.A., M.A. Clay, and P.J. Barter, Remodelling of high density lipoproteins by plasma factors. Atherosclerosis, 1999. 145(2): p. 227-38.

15. van der Velde, A.E., et al., Regulation of direct transintestinal cholesterol excretion in mice. Am J Physiol Gastrointest Liver Physiol, 2008. 295(1): p. G203-g208.

16. Temel, R.E. and J.M. Brown, A new framework for reverse cholesterol transport: nonbiliary contributions to reverse cholesterol transport. World J Gastroenterol, 2010. 16(47): p. 5946-52.

17. Vrins, C.L., et al., Trans-intestinal cholesterol efflux is not mediated through high density lipoprotein. J Lipid Res, 2012. 53(10): p. 2017-23.

18. de la Llera-Moya, M., et al., The ability to promote efflux via ABCA1 determines the capacity of serum specimens with similar high-density lipoprotein cholesterol to remove cholesterol from macrophages. Arterioscler Thromb Vasc Biol, 2010. 30(4): p. 796-801. 
19. Aiello, R.J., D. Brees, and O.L. Francone, ABCA1-deficient mice: insights into the role of monocyte lipid efflux in HDL formation and inflammation. Arterioscler Thromb Vasc Biol, 2003. 23(6): p. 972-80.

20. Hafiane, A. and J. Genest, High density lipoproteins: Measurement techniques and potential biomarkers of cardiovascular risk. BBA Clin, 2015. 3: p. 175-88.

21. Brown, R.J., et al., Cholesterol efflux analyses using stable isotopes and mass spectrometry. Anal Biochem, 2013. 433(1): p. 56-64.

22. Sankaranarayanan, S., et al., A sensitive assay for ABCA1-mediated cholesterol efflux using BODIPY-cholesterol. J Lipid Res, 2011. 52(12): p. 2332-40.

23. Zhang, J., et al., Development of a cell-based, high-throughput screening assay for cholesterol efflux using a fluorescent mimic of cholesterol. Assay Drug Dev Technol, 2011. 9(2): p. 136-46.

24. Robinet, P., et al., A simple and sensitive enzymatic method for cholesterol quantification in macrophages and foam cells. J Lipid Res, 2010. 51(11): p. 3364-9.

25. Holleboom, A.G., et al., In vivo tissue cholesterol efflux is reduced in carriers of a mutation in APOA1. J Lipid Res, 2013. 54(7): p. 1964-71.

26. Turner, S., et al., Measurement of reverse cholesterol transport pathways in humans: in vivo rates of free cholesterol efflux, esterification, and excretion. J Am Heart Assoc, 2012. 1(4): p. e001826.

27. Cuchel, M., et al., A novel approach to measuring macrophage-specific reverse cholesterol transport in vivo in humans. J Lipid Res, 2017. 58(4): p. 752-762.

28. Low, H., A. Hoang, and D. Sviridov, Cholesterol efflux assay. J Vis Exp, 2012(61): p. e3810.

29. Li, X.M., et al., Paradoxical association of enhanced cholesterol efflux with increased incident cardiovascular risks. Arterioscler Thromb Vasc Biol, 2013. 33(7): p. 1696-705.

30. Wang, X., et al., Macrophage ABCA1 and ABCG1, but not SR-BI, promote macrophage reverse cholesterol transport in vivo. J Clin Invest, 2007. 117(8): p. 2216-24.

31. Larrede, S., et al., Stimulation of cholesterol efflux by LXR agonists in cholesterol-loaded human macrophages is ABCA1-dependent but ABCG1-independent. Arterioscler Thromb Vasc Biol, 2009. 29(11): p. 1930-6.

32. Naik, S.U., et al., Pharmacological activation of liver $X$ receptors promotes reverse cholesterol transport in vivo. Circulation, 2006. 113(1): p. 90-7.

33. Bortnick, A.E., et al., The correlation of ATP-binding cassette $1 \mathrm{mRNA}$ levels with cholesterol efflux from various cell lines. J Biol Chem, 2000. 275(37): p. 28634-40.

34. Santamarina-Fojo, S., et al., Regulation and intracellular trafficking of the $A B C A 1$ transporter. J Lipid Res, 2001. 42(9): p. 1339-45.

35. Kilsdonk, E.P., et al., Cellular cholesterol efflux mediated by cyclodextrins. J Biol Chem, 1995. 270(29): p. 17250-6.

36. Zidovetzki, R. and I. Levitan, Use of cyclodextrins to manipulate plasma membrane cholesterol content: evidence, misconceptions and control strategies. Biochim Biophys Acta, 2007. 1768(6): p. 1311-24.

37. Adorni, M.P., et al., The roles of different pathways in the release of cholesterol from macrophages. J Lipid Res, 2007. 48(11): p. 2453-62.

38. Trigueros-Motos, L., et al., ABCA8 Regulates Cholesterol Efflux and High-Density Lipoprotein Cholesterol Levels. Arterioscler Thromb Vasc Biol, 2017. 
39. Sankaranarayanan, S., et al., Serum albumin acts as a shuttle to enhance cholesterol efflux from cells. J Lipid Res, 2013. 54(3): p. 671-6.

40. Pamir, N., et al., Plasminogen promotes cholesterol efflux by the ABCA1 pathway. JCI Insight, 2017. 2(15).

41. Hafiane, A. and J. Genest, ATP binding cassette A1 (ABCA1) mediates microparticle formation during high-density lipoprotein (HDL) biogenesis. Atherosclerosis, 2017. 257: p. 90-99.

42. Davidson, W.S., et al., The effects of apolipoprotein B depletion on HDL subspecies composition and function. J Lipid Res, 2016. 57(4): p. 674-86.

43. Kingwell, B.A., et al., HDL-targeted therapies: progress, failures and future. Nat Rev Drug Discov, 2014. 13(6): p. 445-64.

44. Di Bartolo, B.A., M. Duong, and S.J. Nicholls, Clinical trials with cholesteryl ester transfer protein inhibitors. Curr Opin Lipidol, 2016. 27(6): p. 545-549.

45. Bowman, L., et al., Effects of Anacetrapib in Patients with Atherosclerotic Vascular Disease. N Engl J Med, 2017. 377(13): p. 1217-1227.

46. Kontush, A., M. Lhomme, and M.J. Chapman, Unraveling the complexities of the HDL lipidome. J Lipid Res, 2013. 54(11): p. 2950-63.

47. Asztalos, B.F., M. Tani, and E.J. Schaefer, Metabolic and functional relevance of HDL subspecies. Curr Opin Lipidol, 2011. 22(3): p. 176-85.

48. Krauss, R.M., Lipoprotein subfractions and cardiovascular disease risk. Curr Opin Lipidol, 2010. 21(4): p. 305-11.

49. Camont, L., et al., Small, dense high-density lipoprotein-3 particles are enriched in negatively charged phospholipids: relevance to cellular cholesterol efflux, antioxidative, antithrombotic, anti-inflammatory, and antiapoptotic functionalities. Arterioscler Thromb Vasc Biol, 2013. 33(12): p. 2715-23.

50. Lund-Katz, S., et al., Mechanisms responsible for the compositional heterogeneity of nascent high density lipoprotein. J Biol Chem, 2013. 288(32): p. 23150-60.

51. Gulshan, K., et al., Sphingomyelin depletion impairs anionic phospholipid inward translocation and induces cholesterol efflux. J Biol Chem, 2013. 288(52): p. 37166-79.

52. Darabi, M. and A. Kontush, Can phosphatidylserine enhance atheroprotective activities of high-density lipoprotein? Biochimie, 2016. 120: p. 81-6.

53. Du, X.M., et al., HDL particle size is a critical determinant of ABCA1-mediated macrophage cellular cholesterol export. Circ Res, 2015. 116(7): p. 1133-42.

54. Agarwala, A.P., et al., High-Density Lipoprotein (HDL) phospholipid content and cholesterol efflux capacity are reduced in patients with very high HDL cholesterol and coronary disease. Arterioscler Thromb Vasc Biol, 2015. 35(6): p. 1515-9.

55. Kontush, A., HDL particle number and size as predictors of cardiovascular disease. Front Pharmacol, 2015. 6: p. 218.

56. Arsenault, B.J., et al., HDL particle size and the risk of coronary heart disease in apparently healthy men and women: the EPIC-Norfolk prospective population study. Atherosclerosis, 2009. 206(1): p. 276-81.

57. Favari, E., et al., Small discoidal pre-beta1 HDL particles are efficient acceptors of cell cholesterol via ABCA1 and ABCG1. Biochemistry, 2009. 48(46): p. 11067-74. 
58. Ray, K.K., et al., The effect of cholesteryl ester transfer protein inhibition on lipids, lipoproteins, and markers of HDL function after an acute coronary syndrome: the dalACUTE randomized trial. Eur Heart J, 2014. 35(27): p. 1792-800.

59. Yvan-Charvet, L., et al., Cholesterol efflux potential and antiinflammatory properties of high-density lipoprotein after treatment with niacin or anacetrapib. Arterioscler Thromb Vasc Biol, 2010. 30(7): p. 1430-8.

60. Nicholls, S.J., et al., Assessment of the clinical effects of cholesteryl ester transfer protein inhibition with evacetrapib in patients at high-risk for vascular outcomes: Rationale and design of the ACCELERATE trial. Am Heart J, 2015. 170(6): p. 1061-9.

61. Nicholls, S.J., et al., Cholesterol Efflux Capacity and Pre-Beta-1 HDL Concentrations Are Increased in Dyslipidemic Patients Treated With Evacetrapib. J Am Coll Cardiol, 2015. 66(20): p. 2201-2210.

62. van Capelleveen, J.C., et al., Effects of the cholesteryl ester transfer protein inhibitor, TA8995, on cholesterol efflux capacity and high-density lipoprotein particle subclasses. J Clin Lipidol, 2016. 10(5): p. 1137-1144.e3.

63. Borggreve, S.E., et al., The ability of plasma to stimulate fibroblast cholesterol efflux is associated with the -629C-->A cholesteryl ester transfer protein promoter polymorphism: role of lecithin: cholesterol acyltransferase activity. Biochim Biophys Acta, 2008. 1781(1-2): p. 10-5.

64. McLure, K.G., et al., RVX-208, an inducer of ApoA-I in humans, is a BET bromodomain antagonist. PLoS One, 2013. 8(12): p. e83190.

65. Bailey, D., et al., RVX-208: a small molecule that increases apolipoprotein A-I and highdensity lipoprotein cholesterol in vitro and in vivo. J Am Coll Cardiol, 2010. 55(23): p. 2580-9.

66. Getz, G.S. and C.A. Reardon, Apolipoprotein A-I and A-I mimetic peptides: a role in atherosclerosis. J Inflamm Res, 2011. 4: p. 83-92.

67. Carballo-Jane, E., et al., ApoA-I mimetic peptides promote pre-beta HDL formation in vivo causing remodeling of HDL and triglyceride accumulation at higher dose. Bioorg Med Chem, 2010. 18(24): p. 8669-78.

68. Liao, J.K. and U. Laufs, Pleiotropic effects of statins. Annu Rev Pharmacol Toxicol, 2005. 45: p. 89-118.

69. McTaggart, F. and P. Jones, Effects of statins on high-density lipoproteins: a potential contribution to cardiovascular benefit. Cardiovasc Drugs Ther, 2008. 22(4): p. 321-38.

70. Deedwania, P.C., et al., Effects of rosuvastatin, atorvastatin, simvastatin, and pravastatin on atherogenic dyslipidemia in patients with characteristics of the metabolic syndrome. Am J Cardiol, 2005. 95(3): p. 360-6.

71. Asztalos, B.F., et al., Comparing the effects of five different statins on the HDL subpopulation profiles of coronary heart disease patients. Atherosclerosis, 2002. 164(2): p. 361-9.

72. Asztalos, B.F., et al., Comparison of the effects of high doses of rosuvastatin versus atorvastatin on the subpopulations of high-density lipoproteins. Am J Cardiol, 2007. 99(5): p. 681-5.

73. Ozerova, I.N., et al., Effects of simvastatin on the phospholipid composition of high-density lipoproteins in patients with hypercholesterolemia. Bull Exp Biol Med, 2001. 132(2): p. 763-5. 
74. Shimizu, T., et al., Rosuvastatin activates ATP-binding cassette transporter A1-dependent efflux ex vivo and promotes reverse cholesterol transport in macrophage cells in mice fed a high-fat diet. Arterioscler Thromb Vasc Biol, 2014. 34(10): p. 2246-53.

75. de Vries, R., et al., Cellular cholesterol efflux to plasma from moderately hypercholesterolaemic type 1 diabetic patients is enhanced, and is unaffected by simvastatin treatment. Diabetologia, 2005. 48(6): p. 1105-13.

76. Ronsein, G.E., et al., Niacin Therapy Increases High-Density Lipoprotein Particles and Total Cholesterol Efflux Capacity But Not ABCA1-Specific Cholesterol Efflux in StatinTreated Subjects. Arterioscler Thromb Vasc Biol, 2016. 36(2): p. 404-11.

77. Wang, W., et al., HMG-CoA reductase inhibitors, simvastatin and atorvastatin, downregulate ABCG1-mediated cholesterol efflux in human macrophages. J Cardiovasc Pharmacol, 2013. 62(1): p. 90-8.

78. Niesor, E.J., et al., Statin-induced decrease in ATP-binding cassette transporter A1 expression via microRNA33 induction may counteract cholesterol efflux to high-density lipoprotein. Cardiovasc Drugs Ther, 2015. 29(1): p. 7-14.

79. Franceschini, G., et al., Differential effects of fenofibrate and extended-release niacin on high-density lipoprotein particle size distribution and cholesterol efflux capacity in dyslipidemic patients. J Clin Lipidol, 2013. 7(5): p. 414-22.

80. Brownell, N. and A. Rohatgi, Modulating cholesterol efflux capacity to improve cardiovascular disease. Curr Opin Lipidol, 2016. 27(4): p. 398-407.

81. Tenenbaum, A. and E.Z. Fisman, Fibrates are an essential part of modern antidyslipidemic arsenal: spotlight on atherogenic dyslipidemia and residual risk reduction. Cardiovasc Diabetol, 2012. 11: p. 125.

82. Triolo, M., et al., Simvastatin and bezafibrate increase cholesterol efflux in men with type 2 diabetes. Eur J Clin Invest, 2014. 44(3): p. 240-8.

83. Gardner, C.D., et al., Population frequency distributions of $H D L, H D L(2)$, and $H D L(3)$ cholesterol and apolipoproteins A-I and B in healthy men and women and associations with age, gender, hormonal status, and sex hormone use: the Stanford Five City Project. Prev Med, 2000. 31(4): p. 335-45.

84. Badeau, R.M., et al., Human macrophage cholesterol efflux potential is enhanced by HDLassociated 17beta-estradiol fatty acyl esters. J Steroid Biochem Mol Biol, 2009. 116(1-2): p. 44-9.

85. Pascot, A., et al., HDL particle size: a marker of the gender difference in the metabolic risk profile. Atherosclerosis, 2002. 160(2): p. 399-406.

86. Catalano, G., et al., Cellular SR-BI and ABCA1-mediated cholesterol efflux are genderspecific in healthy subjects. J Lipid Res, 2008. 49(3): p. 635-43.

87. Li, Z., et al., Effects of gender and menopausal status on plasma lipoprotein subspecies and particle sizes. J Lipid Res, 1996. 37(9): p. 1886-96.

88. Stevenson, J.C., D. Crook, and I.F. Godsland, Influence of age and menopause on serum lipids and lipoproteins in healthy women. Atherosclerosis, 1993. 98(1): p. 83-90.

89. Eapen, D.J., et al., Raising HDL cholesterol in women. Int J Womens Health, 2010. 1: p. 181-91.

90. Corcoran, M.P., et al., The effect of 17beta-estradiol on cholesterol content in human macrophages is influenced by the lipoprotein milieu. J Mol Endocrinol, 2011. 47(1): p. 109117. 
91. Badeau, R.M., et al., The impact of gender and serum estradiol levels on HDL-mediated reverse cholesterol transport. Eur J Clin Invest, 2013. 43(4): p. 317-23.

92. Badeau, R.M., et al., High-density lipoprotein-associated 17beta-estradiol fatty acyl ester uptake by Fu5AH hepatoma cells: implications of the roles of scavenger receptor class B, type I and the low-density lipoprotein receptor. Biochim Biophys Acta, 2007. 1771(10): p. 132934.

93. El Khoudary, S.R., et al., Cholesterol efflux capacity and subclasses of HDL particles in healthy women transitioning through menopause. J Clin Endocrinol Metab, 2016: p. jc20162144.

94. Pascot, A., et al., Reduced HDL particle size as an additional feature of the atherogenic dyslipidemia of abdominal obesity. J Lipid Res, 2001. 42(12): p. 2007-14.

95. Williams, P.T., et al., Associations of age, adiposity, alcohol intake, menstrual status, and estrogen therapy with high-density lipoprotein subclasses. Arterioscler Thromb, 1993. 13(11): p. 1654-61.

96. Varady, K.A., et al., Comparison of effects of diet versus exercise weight loss regimens on LDL and HDL particle size in obese adults. Lipids Health Dis, 2011. 10: p. 119.

97. Magkos, F., B.S. Mohammed, and B. Mittendorfer, Effect of obesity on the plasma lipoprotein subclass profile in normoglycemic and normolipidemic men and women. Int J Obes (Lond), 2008. 32(11): p. 1655-64.

98. Rashid, S. and J. Genest, Effect of obesity on high-density lipoprotein metabolism. Obesity (Silver Spring), 2007. 15(12): p. 2875-88.

99. Sasahara, T., et al., Altered properties of high density lipoprotein subfractions in obese subjects. J Lipid Res, 1997. 38(3): p. 600-11.

100. Ding, J., et al., Alterations of a Cellular Cholesterol Metabolism Network Are a Molecular Feature of Obesity-Related Type 2 Diabetes and Cardiovascular Disease. Diabetes, 2015. 64(10): p. 3464-74.

101. Sasahara, T., et al., Cholesterol transport between cells and high density lipoprotein subfractions from obese and lean subjects. J Lipid Res, 1998. 39(3): p. 544-54.

102. Autran, D., et al., Basal and postprandial serum-promoted cholesterol efflux in normolipidemic subjects: Importance of fat mass distribution. Metabolism, 2001. 50(11): p. 1330-5.

103. Attia, N., et al., Impact of android overweight or obesity and insulin resistance on basal and postprandial SR-BI and ABCA1-mediated serum cholesterol efflux capacities. Atherosclerosis, 2010. 209(2): p. 422-9.

104. Mantyselka, P., et al., Weight change and lipoprotein particle concentration and particle size: a cohort study with 6.5-year follow-up. Atherosclerosis, 2012. 223(1): p. 239-43.

105. Asztalos, B.F., et al., Effects of weight loss, induced by gastric bypass surgery, on HDL remodeling in obese women. J Lipid Res, 2010. 51(8): p. 2405-12.

106. Aron-Wisnewsky, J., et al., Effect of bariatric surgery-induced weight loss on SR-BI-, $A B C G 1-$, and ABCA1-mediated cellular cholesterol efflux in obese women. J Clin Endocrinol Metab, 2011. 96(4): p. 1151-9.

107. Davidson, W.S., et al., Weight loss surgery in adolescents corrects high-density lipoprotein subspecies and their function. Int J Obes (Lond), 2017. 41(1): p. 83-89. 
108. Osto, E., et al., Rapid and body weight-independent improvement of endothelial and highdensity lipoprotein function after Roux-en-Y gastric bypass: role of glucagon-like peptide-1. Circulation, 2015. 131(10): p. 871-81.

109. Wang, Y., et al., Prolonged caloric restriction in obese patients with type 2 diabetes mellitus decreases plasma CETP and increases apolipoprotein AI levels without improving the cholesterol efflux properties of HDL. Diabetes Care, 2011. 34(12): p. 2576-80.

110. Aicher, B.O., et al., Diet-induced weight loss in overweight or obese women and changes in high-density lipoprotein levels and function. Obesity (Silver Spring), 2012. 20(10): p. 2057-62.

111. Vasudevan, M., et al., Modest diet-induced weight loss reduces macrophage cholesterol efflux to plasma of patients with metabolic syndrome. J Clin Lipidol, 2013. 7(6): p. 661-70.

112. Martinez-Ramirez, M., et al., HDL-sphingomyelin reduction after weight loss by an energy-restricted diet is associated with the improvement of lipid profile, blood pressure, and decrease of insulin resistance in overweight/obese patients. Clin Chim Acta, 2016. 454: p. 77-81.

113. North, B.J. and D.A. Sinclair, The intersection between aging and cardiovascular disease. Circ Res, 2012. 110(8): p. 1097-108.

114. Walter, M., Interrelationships among HDL metabolism, aging, and atherosclerosis. Arterioscler Thromb Vasc Biol, 2009. 29(9): p. 1244-50.

115. Luc, G., et al., High-density lipoprotein particles in octogenarians. Metabolism, 1991. 40(12): p. 1238-43.

116. Mutharasan, R.K., et al., HDL efflux capacity, HDL particle size, and high-risk carotid atherosclerosis in a cohort of asymptomatic older adults: the Chicago Healthy Aging Study. J Lipid Res, 2017. 58(3): p. 600-606.

117. Suc, I., et al., Oxidative tyrosylation of high density lipoproteins impairs cholesterol efflux from mouse J774 macrophages: role of scavenger receptors, classes A and B. J Cell Sci, 2003. 116(Pt 1): p. 89-99.

118. Berrougui, H., et al., Age-related impairment of HDL-mediated cholesterol efflux. J Lipid Res, 2007. 48(2): p. 328-36.

119. Khalil, A., J.P. Jay-Gerin, and T. Fulop, Jr., Age-related increased susceptibility of highdensity lipoproteins (HDL) to in vitro oxidation induced by gamma-radiolysis of water. FEBS Lett, 1998. 435(2-3): p. 153-8.

120. Sene, A., et al., Impaired cholesterol efflux in senescent macrophages promotes age-related macular degeneration. Cell Metab, 2013. 17(4): p. 549-61.

121. Rayner, K.J., et al., MiR-33 contributes to the regulation of cholesterol homeostasis. Science, 2010. 328(5985): p. 1570-3.

122. Annema, W., et al., ApoE promotes hepatic selective uptake but not RCT due to increased ABCA1-mediated cholesterol efflux to plasma. J Lipid Res, 2012. 53(5): p. 929-40.

123. Holzer, M., et al., Aging affects high-density lipoprotein composition and function. Biochim Biophys Acta, 2013. 1831(9): p. 1442-8.

124. Hoang, A., et al., ABCA1 expression in humans is associated with physical activity and alcohol consumption. Atherosclerosis, 2008. 197(1): p. 197-203.

125. Olchawa, B., et al., Physical fitness and reverse cholesterol transport. Arterioscler Thromb Vasc Biol, 2004. 24(6): p. 1087-91. 
126. Escola-Gil, J.C., et al., HDL and lifestyle interventions. Handb Exp Pharmacol, 2015. 224: p. 569-92.

127. Freeman, D.J., et al., Smoking and plasma lipoproteins in man: effects on low density lipoprotein cholesterol levels and high density lipoprotein subfraction distribution. Eur J Clin Invest, 1993. 23(10): p. 630-40.

128. Song, W., et al., The implication of cigarette smoking and cessation on macrophage cholesterol efflux in coronary artery disease patients. J Lipid Res, 2015. 56(3): p. 682-91.

129. Beulens, J.W., et al., Moderate alcohol consumption increases cholesterol efflux mediated by ABCA1. J Lipid Res, 2004. 45(9): p. 1716-23.

130. Syeda, F., et al., Postprandial variations in the cholesteryl ester transfer protein activity, phospholipid transfer protein activity and plasma cholesterol efflux capacity in normolipidemic men. Nutr Metab Cardiovasc Dis, 2003. 13(1): p. 28-36.

131. Berryman, C.E., et al., Acute consumption of walnuts and walnut components differentially affect postprandial lipemia, endothelial function, oxidative stress, and cholesterol efflux in humans with mild hypercholesterolemia. J Nutr, 2013. 143(6): p. 788-94.

132. Bechor, S., et al., 9-cis beta-Carotene Increased Cholesterol Efflux to HDL in Macrophages. Nutrients, 2016. 8(7).

133. Afonso Mda, S., et al., The impact of dietary fatty acids on macrophage cholesterol homeostasis. J Nutr Biochem, 2014. 25(2): p. 95-103.

134. Buonacorso, V., et al., Macrophage cholesterol efflux elicited by human total plasma and by $H D L$ subfractions is not affected by different types of dietary fatty acids. Am J Clin Nutr, 2007. 86(5): p. 1270-7.

135. Kralova Lesna, I., et al., Replacement of dietary saturated FAs by PUFAs in diet and reverse cholesterol transport. J Lipid Res, 2008. 49(11): p. 2414-8.

136. Wang, Y. and J.F. Oram, Unsaturated fatty acids inhibit cholesterol efflux from macrophages by increasing degradation of ATP-binding cassette transporter A1. J Biol Chem, 2002. 277(7): p. 5692-7.

137. Kasbi Chadli, F., et al., Omega 3 fatty acids promote macrophage reverse cholesterol transport in hamster fed high fat diet. PLoS One, 2013. 8(4): p. e61109.

138. Tanaka, N., et al., Administration of high dose eicosapentaenoic acid enhances antiinflammatory properties of high-density lipoprotein in Japanese patients with dyslipidemia. Atherosclerosis, 2014. 237(2): p. 577-83.

139. Ringseis, R., et al., Conjugated linoleic acid isomers reduce cholesterol accumulation in acetylated LDL-induced mouse RAW264.7 macrophage-derived foam cells. Lipids, 2008. 43(10): p. 913-23.

140. Helal, O., et al., Extra-virgin olive oil consumption improves the capacity of HDL to mediate cholesterol efflux and increases $A B C A 1$ and $A B C G 1$ expression in human macrophages. $\mathrm{Br}$ J Nutr, 2013. 109(10): p. 1844-55.

141. Farras, M., et al., Olive oil polyphenols enhance the expression of cholesterol efflux related genes in vivo in humans. A randomized controlled trial. J Nutr Biochem, 2013. 24(7): p. 1334-9.

142. Hernaez, A., et al., Olive oil polyphenols enhance high-density lipoprotein function in humans: a randomized controlled trial. Arterioscler Thromb Vasc Biol, 2014. 34(9): p. 2115-9. 
143. Hernaez, A., et al., Mediterranean Diet Improves High-Density Lipoprotein Function in High-Cardiovascular-Risk Individuals: A Randomized Controlled Trial. Circulation, 2017. 135(7): p. 633-643.

144. Villard, E.F., et al., Genetic determination of plasma cholesterol efflux capacity is genderspecific and independent of HDL-cholesterol levels. Arterioscler Thromb Vasc Biol, 2013. 33(4): p. 822-8.

145. Koekemoer, A.L., et al., Large-Scale Analysis of Determinants, Stability, and Heritability of High-Density Lipoprotein Cholesterol Efflux Capacity. Arterioscler Thromb Vasc Biol, 2017. 37(10): p. 1956-1962.

146. Ronsein, G.E. and T. Vaisar, Inflammation, remodeling, and other factors affecting HDL cholesterol efflux. Curr Opin Lipidol, 2017. 28(1): p. 52-59.

147. Tall, A.R. and L. Yvan-Charvet, Cholesterol, inflammation and innate immunity. Nat Rev Immunol, 2015. 15(2): p. 104-16.

148. Eren, E., N. Yilmaz, and O. Aydin, High Density Lipoprotein and it's Dysfunction. Open Biochem J, 2012. 6: p. 78-93.

149. Vaisar, T., et al., Inflammatory remodeling of the HDL proteome impairs cholesterol efflux capacity. J Lipid Res, 2015. 56(8): p. 1519-30.

150. McGillicuddy, F.C., et al., Inflammation impairs reverse cholesterol transport in vivo. Circulation, 2009. 119(8): p. 1135-45.

151. de Beer, M.C., et al., ATP binding cassette G1-dependent cholesterol efflux during inflammation. J Lipid Res, 2011. 52(2): p. 345-53.

152. Wang, Z. and T. Nakayama, Inflammation, a link between obesity and cardiovascular disease. Mediators Inflamm, 2010. 2010: p. 535918.

153. Voloshyna, I. and S. Seshadri, Infliximab reverses suppression of cholesterol efflux proteins by TNF-alpha: a possible mechanism for modulation of atherogenesis. 2014. 2014: p. 312647.

154. Petelin, A., et al., Low-grade inflammation in overweight and obese adults is affected by weight loss program. J Endocrinol Invest, 2014. 37(8): p. 745-55.

155. Rohatgi, A., et al., HDL cholesterol efflux capacity and incident cardiovascular events. N Engl J Med, 2014. 371(25): p. 2383-93.

156. Ishikawa, T., et al., High-density lipoprotein cholesterol efflux capacity as a relevant predictor of atherosclerotic coronary disease. Atherosclerosis, 2015. 242(1): p. 318-22. 


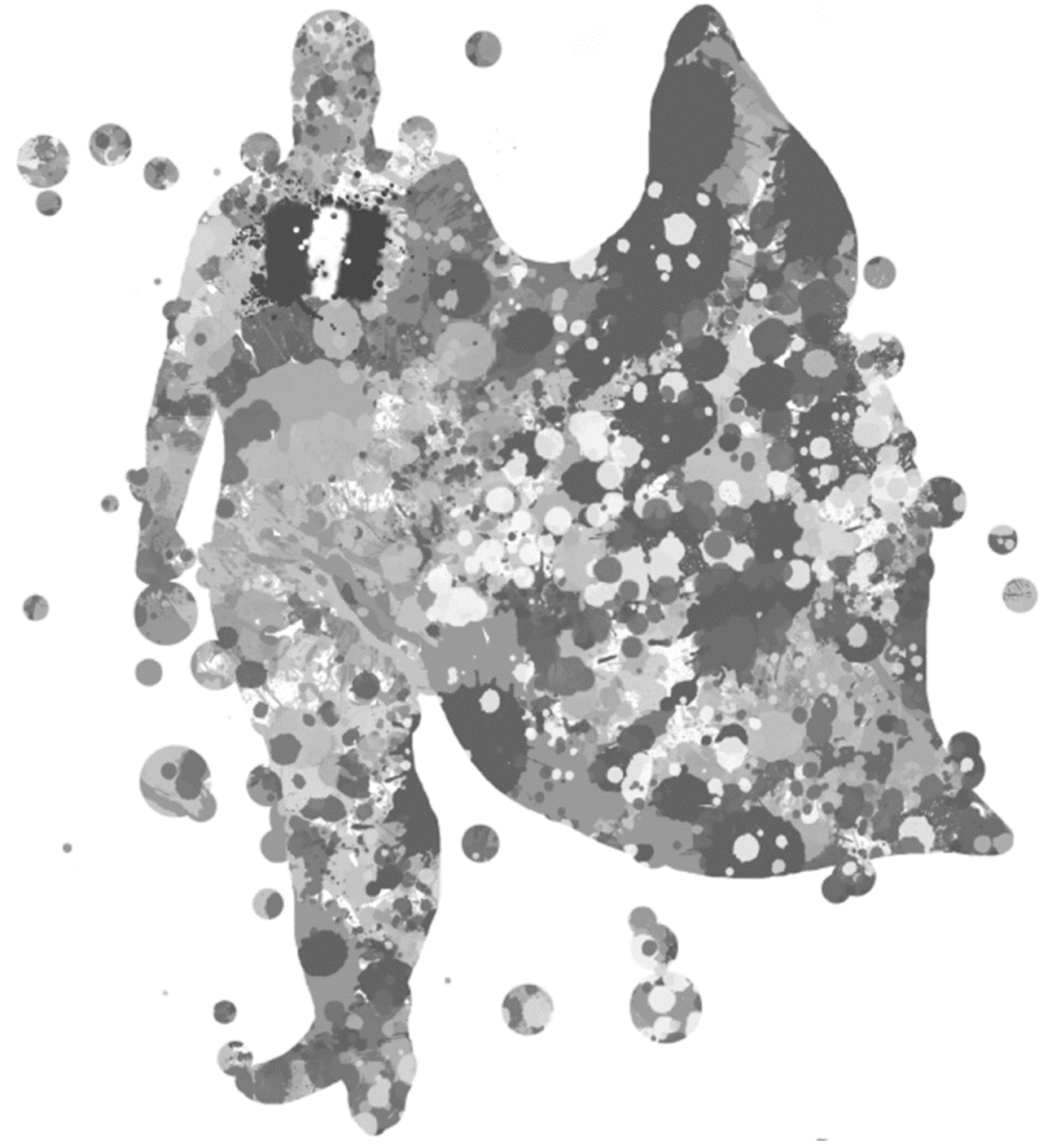




\section{Chapter 3}

\section{SNPs located in ABCA1, ABCG1,}

$L X R \beta$ and CETP are associated with plasma cholesterol efflux

Charlotte P.J. Talbot, Jogchum Plat, Herman E. Popeijus, Andreas Ritsch,

Ronald P. Mensink

In preparation 


\begin{abstract}
Associations between cholesterol efflux capacity of high-density lipoprotein (HDL) particles with subject characteristics and selected Single Nucleotide Polymorphisms (SNPs) of genes involved in the reverse cholesterol transport pathway and HDL metabolism have been investigated in 50 healthy men and 57 healthy women. Cholesterol efflux was measured by incubating radiolabeled- ${ }^{3} \mathrm{H}$-cholesterol loaded murine J774 macrophages with apolipoprotein B-depleted serum. Results were adjusted for the use of statins, age and HDL-C concentrations. Cholesterol efflux was significantly associated with the use of statins ( $\beta=17.7 \%$; $p=0.014)$ and HDL-C concentrations $(\mathrm{mmol} / \mathrm{L})(\beta=20.4 \% ; p=0.012)$, and a trend was observed for age (years) $(\beta=-0.3 \% ; p=0.083)$. No associations were found for gender and BMI. Cholesterol efflux capacity was related to SNPs in ABCA1 (rs2066716), LXR $\beta$ (rs2248949 and rs2695121) and CETP (rs1864163). A trend was found for ABCG1 (rs2839482). No relationships were present for SNPs in the apoA5/A4/C3/A1 cluster and the selected SNP (rs5888) in SRB1. Since these proteins are not all present in the HDL particles added to the murine J774 macrophages, the identified SNPs may be related to HDL particles characteristics.
\end{abstract}




\section{Introduction}

Numerous epidemiological studies have found an inverse association between serum high-density lipoprotein-cholesterol (HDL-C) concentrations and cardiovascular disease (CVD) risk [1,2]. However, a causal relation between increasing serum HDL-C concentrations and CVD is not supported by recent intervention studies [3]. Therefore, it becomes more and more accepted that improving HDL functionality is a better target for decreasing CVD risk than increasing HDL-C concentrations. Indeed, HDL possesses many anti-atherogenic properties, including anti-inflammatory, anti-oxidant, anti-apoptotic, antithrombotic and vasodilating effects [4]. However, one of the main anti-atherogenic properties of HDL is its ability to promote cholesterol efflux from macrophages, which is the transport of cholesterol from peripheral cells, predominantly macrophages, back to the liver and its subsequent excretion into the feces as neutral sterols or bile acids. This ability of HDL to stimulate efflux of cholesterol from macrophages is part of the reverse cholesterol transport (RCT) pathway [4]. Indeed, in vitro cholesterol efflux capacity from macrophages mediated by HDL particles was negatively associated with the development of CVD and atherosclerosis, independently of HDL-C concentrations, highlighting the importance of focusing on HDL functionality rather than HDL-C concentrations [5, 6].

Plasma HDL-C concentrations are largely genetically determined and approximately $70 \%$ of the inter-individual variation in plasma HDL-C concentrations is explained by genetics factors [7]. In fact, many studies have reported that single nucleotide polymorphisms (SNPs) in genes involved in HDL metabolism, such as cholesteryl ester transfer protein (CETP), lipoprotein lipase $(L P L)$, hepatic lipase (LIPC), endothelial lipase (LIPG) and ATP-binding cassette 1 $(A B C A 1)[7,8]$ are associated with HDL-C and apolipoprotein A-I (apoA1) concentrations [9, 10]. However, relationships between SNPs and HDL functionality, and more especially with the cholesterol efflux capacity of HDL particles, are not well documented. In one study, it was found that plasma cholesterol efflux capacity from human macrophages was related to SNPs located in genes coding for CETP, ABCA1, apoA1, apolipoprotein A-II and LIPC. Effects were gender specific and independent of plasma lipid concentrations. However, in that study, only genes involved in the biogenesis, maturation and remodeling of HDL particles were examined [11]. In the present study, we therefore decided to focus on genes directly involved in cholesterol efflux and the RCT pathway. For this purpose, we investigated in 50 healthy men and 57 healthy women associations between SNPs located in genes coding for $A B C A 1$, ATP binding cassette transporter G1 (ABCG1), liver $\mathrm{X}$ receptor (LXR), scavenger receptor class $\mathrm{B}$ member 1 (SRB1), CETP and the apolipoprotein A5/A4/C3/A1 cluster (apoA5/A4/C3/A1) with plasma 
cholesterol efflux capacity. Also, relations with age, gender, body mass index (BMI), and use of statins were explored. Although these factors are related to HDL-C concentrations [12-15], not much is known on their relationships with cholesterol efflux capacity of HDL particles.

\section{Materials and Methods}

\section{Subjects' characteristics}

Samples from two previous studies carried out at Maastricht University were used. In the first study, effects of vegetable-oil based versus wood-based stanol ester mixtures on serum lipids and hemostatic factors in non-hypercholesterolemic subjects were studied. Of the original 112 healthy subjects, samples were available for 24 men and 42 women. All subjects were healthy with a BMI $<30 \mathrm{~kg} / \mathrm{m}^{2}$ and aged between 18-65 years. Full details of the study have been published before [16]. In the second study, the effects of long-term plant sterol or stanol ester consumption on lipid and lipoprotein metabolism were examined in subjects on stable statin treatment. Of the original 54 subjects, samples of 26 men and 15 women were available. All subjects were healthy with a BMI $\leq 32 \mathrm{~kg} / \mathrm{m}^{2}$ and aged between 18-65 years. The types of statin used by the subjects are shown in Supplemental Table S1. Full details of the study have been published before [17]. For the present study, results of both studies obtained at the end of the 4 weeks run-in periods were combined and associations with cholesterol efflux capacity could therefore be determined in 50 healthy men and 57 healthy women. Both studies were conducted according to the ethical guidelines of the 1975 Declaration of Helsinki and approved by the Ethics Committee of Maastricht University Medical Centre. Written informed consent was obtained from all participants before entering the study.

In the first study, subjects had to replace their own spreads during the 4 weeks runin period by a low-erucic acid rapeseed-oil based margarine (74\% absorbable fat), of which minimal $20 \mathrm{~g}$ per day had to be consumed. For the second study, the participants had to replace their own spread with the experimental 'light' margarine ( $40 \%$ absorbable fat), of which $30 \mathrm{~g}$ per day had to be consumed. In both studies, fasting blood samples were collected by venipuncture at the end of the 4 weeks runin-periods. Serum and EDTA plasma were obtained by centrifugation at $2000 \mathrm{~g}$ for 30 minutes at $4^{\circ} \mathrm{C}$, at least 1 hour after venipuncture, and at $2000 \mathrm{~g}$ for 30 minutes directly after sampling, respectively. Samples were stored at $-80^{\circ} \mathrm{C}$ until use. 


\section{Biochemical Measurements}

Serum HDL-C concentrations were determined after precipitation of apoBcontaining lipoproteins by adding a mixture of phosphotungstic acid and magnesium ions to the sample (CHOD/PAP method; Roche Diagnostics System, Hofmann-La Roche Ltd., Basel, Swiss). Serum apoA1 concentrations were measured using an immunoturbidimetric reaction (UNI-KIT ApoA1, Roche, Basel). Serum triacylglycerol was analyzed with correction for free glycerol concentrations (GPOTrinder; Sigma Diagnostics, St Louis, USA). All samples from one subject were analyzed within the same run.

The ability of the HDL particles to promote cholesterol efflux from macrophages was measured as previously described [18]. Briefly, murine J774 macrophages were first cultivated in the presence of an acyl-coenzyme A:cholesterol acyltransferase (ACAT) inhibitor to block cholesterol esterification. The macrophages were radiolabeled with $2.5 \mu \mathrm{Ci}{ }^{3} \mathrm{H}$-cholesterol and $-24 \mathrm{~h}$ after labeling - activated with cAMP in order to upregulate the efflux transporter ABCA1. Subsequently, apolipoprotein B containing lipoproteins from the human serum samples were precipitated using tungstophosphoric acid hydrate and magnesium chloride. After spinning, the supernatant containing the HDL particles was added to the radiolabeled macrophages and incubated for $4 \mathrm{~h}$. All steps were performed in the presence of the ACAT inhibitor. The amount of efflux of radioactive cholesterol from the cells was quantified by using liquid scintillation counting (\% of the efflux of a standard serum). Three pooled serum controls from healthy subjects were included in each assay, and the efflux capacity values obtained for each subject were normalized to the average efflux capacity value of these pooled sera, which was set to $100 \%$. Each sample was analyzed in triplicate. Proteins involved in cholesterol efflux from macrophages, such as $A B C A 1, A B C G 1$ and $S R B 1$ are an intrinsic part of the assay as they are part of the J774 macrophages and therefore similar in all analyses. However, the HDL fraction added to these cells is subject-specific.

CETP mass was quantified in plasma samples with an enzyme-linked immunosorbent assay (ELISA) using a CETP-specific recombinant single-chain antibody as coating antibody and an affinity-purified polyclonal anti-CETP antibody as detection antibody, as previously described [19]. CETP-activity measurements were performed by incubating whole plasma with radiolabeled HDL and the ability of exogenous HDL to transfer cholesteryl ester (CE) to apoBcontaining lipoproteins was measured as previously described [20, 21]. Cholesteryl ester transfer was measured as the fraction of the total radiolabeled-CE transferred to apoB-containing lipoproteins compared with controls. Results were expressed as the percentage decrease of ${ }^{3} \mathrm{H}-\mathrm{CE}$ in the supernatant of total radiolabeled-CE. 


\section{SNP analysis}

Genomic DNA was isolated from leukocytes, using QIAamp genomic DNA isolation Kit (Qiagen, Hilden, Germany) according to manufacturer's instructions. In each sample, 26 SNPs located in genes involved in the RCT pathway ( $A B C A 1$, $A B C G 1, L X R, S R B 1, C E T P$ and the apoA5/A4/C3/A1 cluster) were analyzed using custom made GoldenGate BeadChips following the GoldenGate Genotyping Assay manual (Illumina, San Diego, USA). Loci names and their functional consequences are shown in Supplemental Table S2.

\section{Statistical analysis}

First, differences in baseline characteristics between men and women were examined using an independent t-test. Then, relationships between cholesterol efflux capacity with BMI and use of statins were analyzed using an independent $\mathrm{t}$ test. For BMI, subjects were categorized into normal weight subjects (BMI $<25$ $\mathrm{kg} / \mathrm{m}^{2}$ ) and overweight and obese subjects (BMI $\geq 25 \mathrm{~kg} / \mathrm{m}^{2}$ ). Pearson correlation coefficients between plasma cholesterol efflux capacity with age and HDL-C were also calculated. Finally, all these variables (gender, BMI-group, use of statins, age and HDL-C concentrations) were included into one multiple linear regression model to examine the independent contributions of these factors to plasma cholesterol efflux capacity. Variables with a P-value $<0.1$ were included into subsequent analyses.

Multiple linear regression analysis was also used to study associations between plasma cholesterol efflux with parameters related to HDL metabolism (total cholesterol, LDL-C, triacylglycerol and apoA1 concentrations, CETP-mass and CETP-activity) after adjusting for the use of statins and age.

Relationships between SNPs with cholesterol efflux capacity were analyzed using the General Linear Model (GLM) procedure. Results were adjusted for the factors use of statins, age and HDL-C concentrations. If a SNP reached statistical significance, a Bonferroni correction was applied to compare the three genotypegroups of a SNP pairwise. Based on these results, a dominant or a recessive association model was build with adjustments for the variables use of statins, age and HDL-C concentrations. A dominant model was used if the least frequent homozygous genotype (e.g. $\mathrm{mm}$ ) showed a comparable efflux capacity than the heterozygous genotype (e.g. $\mathrm{mM}$ ). In that case, $\mathrm{mm}+\mathrm{mM}$ were compared with $\mathrm{MM}$. Similarly, in a recessive model mm was compared with $\mathrm{mM}+\mathrm{MM}$. A $p$-value $\leq 0.05$ was considered significant. All statistical analyses were performed with SPSS 20.0 for Mac OS. 


\section{Results}

\section{Association between subject characteristics with plasma cholesterol efflux capacity}

Among the study population, $26.3 \%$ of the women and $52.0 \%$ of the men were using statins. Table 1 shows that BMI, total cholesterol and LDL-C concentrations were comparable between men and women. In contrast, HDL-C $(+28 \% ; p \leq 0.001)$ and apoA1 concentrations ( $+19 \% ; p \leq 0.001)$, CETP mass $(+19 \% ; p=0.007)$ and cholesterol efflux capacity $(+11 \%$; $p=0.039)$ were significantly higher in women as compared with men, whereas cholesteryl ester transfer $(-4 \% ; p=0.027)$ and triacylglycerol concentrations $(-25 \% ; p=0.012)$ were significantly lower in women. Moreover, women were on average 10 years younger as compared with men $(p=0.001)$. Cholesterol efflux in normal-weight subjects (BMI $<25 \mathrm{~kg} / \mathrm{m}^{2} ; 22.2 \pm 2.1 \mathrm{~kg} / \mathrm{m}^{2} ; \mathrm{n}=70$ ) was $93.8 \pm 25.9 \%$ and comparable to the value of $95.1 \pm 23.2 \%$ in overweight and obese subjects (BMI $\geq 25 \mathrm{~kg} / \mathrm{m} 2 ; 26.7 \pm 5.0 \mathrm{~kg} / \mathrm{m}^{2} ; \mathrm{n}=37 ; p=0.791$ for the difference). Cholesterol efflux capacity was similar between the subjects using statins $(96.6 \pm$ $25.3 \% ; n=41)$ as compared to subjects without a statin treatment $(92.7 \pm 24.7 \% ; n=66$; $p=0.430$ for the difference). Moreover, cholesterol efflux was not correlated with the age $(\mathrm{r}=-0.74 ; p=0.448)$. However, multiple linear regression analysis showed that the use of statins $(\beta=17.7 ; p=0.014)$ and HDL-C concentrations $(\mathrm{mmol} / \mathrm{L})(\beta=20.4 ; p=0.012)$ were significantly related to plasma cholesterol efflux capacity, while a tendency was observed for age (years) $(\beta=-0.3 ; p=0.083)$. No associations with gender (men vs. women: $\beta=-3.7 ; p=0.496$ ) and BMI-group (high vs. low: $\beta=7.4 ; p=0.187$ ) were found. Supplemental Table S1 shows that after adjustment for age and HDL-C concentrations, subjects using atorvastatin $(107.1 \pm 22.8 \% ; n=10 ; p=0.032)$ had a significantly higher cholesterol efflux capacity as compared to non-statins users $(92.7$ $\pm 24.7 \%$; $\mathrm{n}=66$ ). No differences between the other types of statin with the non-statins users were found. After adjusting for the use of statins and age, multiple linear regression analysis showed that cholesterol efflux was associated with apoA1 $(\mathrm{g} / \mathrm{L})$ $(\beta=28.3 \% ; p=0.003)$ and total cholesterol concentrations $(\mathrm{mmol} / \mathrm{L})(\beta=6.8 \% ; \mathrm{p}=0.048)$, but not with LDL-C (mmol/L) $(\beta=1.7 \% ; \mathrm{p}=0.655)$ and triacylglycerol concentrations $(\mathrm{mmol} / \mathrm{L})(\beta=1.4 \% ; p=0.724)$, CETP-mass $(\mu \mathrm{g} / \mathrm{mL})(\beta=8.1 \% ; p=0.140)$ or CETP-activity (\%) $(\beta=-0.1 ; \mathrm{p}=0.773)$. 
Table 1: Baseline characteristics of the study population

\begin{tabular}{|c|c|c|c|c|c|c|c|c|c|}
\hline & \multicolumn{3}{|c|}{$\begin{array}{l}\text { All subjects } \\
(\mathrm{n}=107)\end{array}$} & \multicolumn{3}{|c|}{$\begin{array}{c}\text { Men } \\
(n=50)\end{array}$} & \multicolumn{3}{|c|}{$\begin{array}{c}\text { Women } \\
(n=57)\end{array}$} \\
\hline Use of statins (\%) & \multicolumn{3}{|c|}{38.3} & \multicolumn{3}{|c|}{52} & \multicolumn{3}{|c|}{26.3} \\
\hline Age (years) & 42.8 & \pm & 18.0 & 48.7 & \pm & 16.6 & 37.6 & \pm & $17.7^{* * *}$ \\
\hline Body mass index $\left(\mathrm{kg} / \mathrm{m}^{2}\right)$ & 23.7 & \pm & 4.0 & 23.9 & \pm & 4.6 & 23.5 & \pm & 3.4 \\
\hline Total Cholesterol (mmol/L) & 5.10 & \pm & 0.76 & 5.05 & \pm & 0.75 & 5.15 & \pm & 0.77 \\
\hline LDL-C (mmol/L) & 3.02 & \pm & 0.71 & 3.09 & \pm & 0.70 & 2.96 & \pm & 0.72 \\
\hline HDL-C (mmol/L) & 1.55 & \pm & 0.37 & 1.35 & \pm & 0.29 & 1.73 & \pm & $0.34^{* * *}$ \\
\hline Triacylglycerol (mmol/L) & 1.16 & \pm & 0.69 & 1.34 & \pm & 0.75 & 1.01 & \pm & $0.60^{* *}$ \\
\hline Apolipoprotein A1 (g/L) & 1.64 & \pm & 0.27 & 1.49 & \pm & 0.19 & 1.78 & \pm & $0.26^{* * *}$ \\
\hline CETP mass $(\mu \mathrm{g} / \mathrm{mL})$ & 1.34 & \pm & 0.45 & 1.22 & \pm & 0.40 & 1.45 & \pm & $0.47^{* *}$ \\
\hline Cholesteryl ester transfer (\%) & 59.9 & \pm & 5.2 & 61.1 & \pm & 4.0 & 58.8 & \pm & $6.0^{*}$ \\
\hline Cholesterol efflux capacity (\%) & 94.2 & \pm & 24.9 & 88.9 & \pm & 22.5 & 98.8 & \pm & $26.1^{*}$ \\
\hline
\end{tabular}

Values are means $\pm S D$

Significantly different between men and women: ${ }^{*} p \leq 0.05,{ }^{* *} p \leq 0.01,{ }^{* * *} p \leq 0.001$

\section{Association between genetics polymorphism with plasma cholesterol efflux capacity}

The genotype distribution and the relationship between the selected SNPs coding for genes involved in the RCT pathway with cholesterol efflux capacity are reported in Table 2. Plasma cholesterol efflux capacity was significantly associated with 1 SNP in $A B C A 1$ (rs2066716; $p=0.015$ ), 2 SNPs in $L X R \beta$ (rs2248949 and rs2695121; both $p \leq 0.001$ ) and 1 SNP in CETP (rs1864163; $p=0.026)$. A trend was found for 1 SNP in $A B C G 1$ (rs2839482; $p=0.074$ ), while no relationships were found with the analyzed SNPs in the apoA5/A4/C3/A1 cluster or SRB1 gene. 


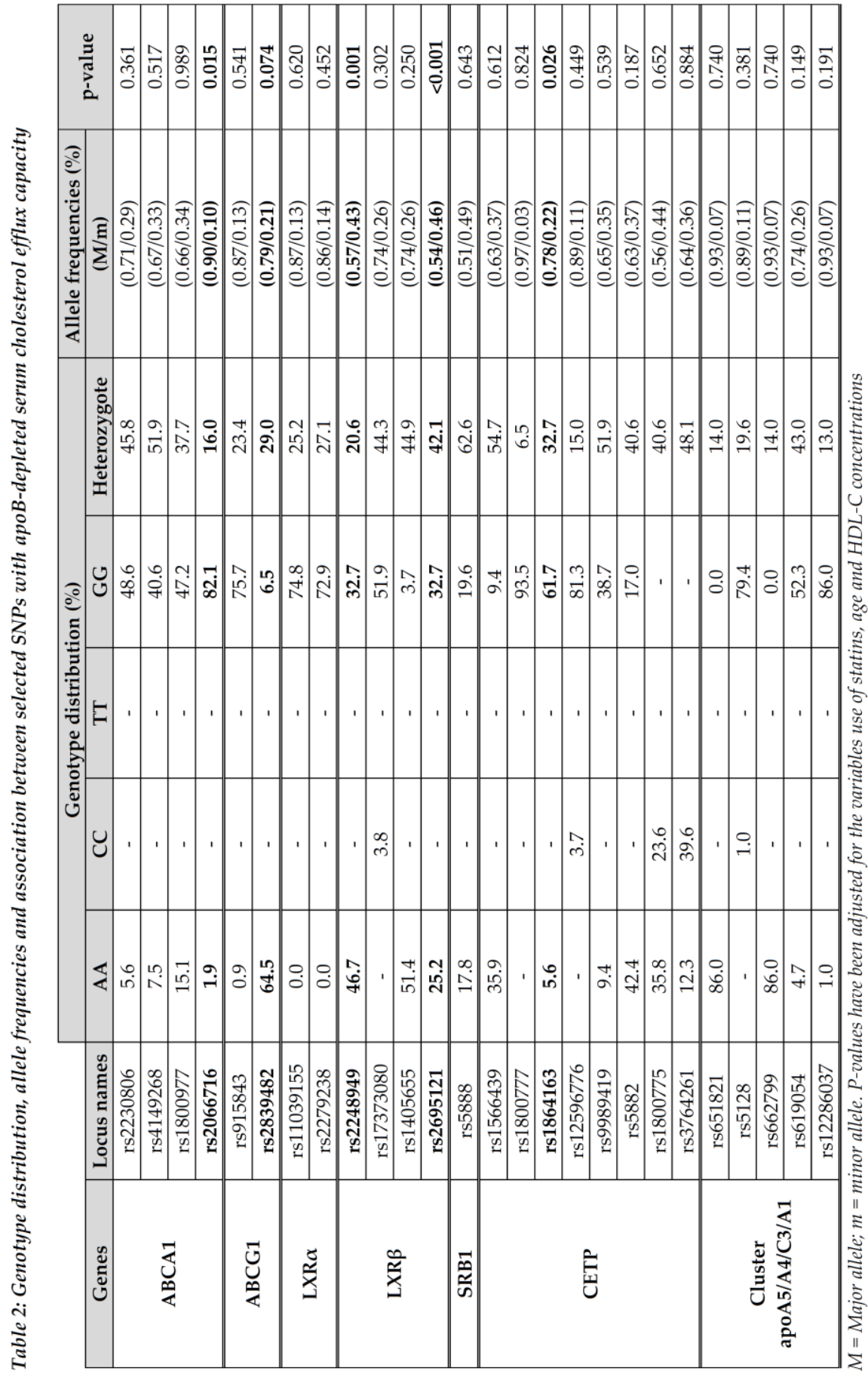


Next, multiple regression analysis was performed using a recessive or a dominant model for the 5 SNPs with a $p$-value $<0.1$. For $A B C A 1$ (rs2066716) a dominant model was used, as only 2 subjects $(1.9 \%)$ were carriers of the minor genotype AA. An increased capacity to mediate cholesterol efflux from human macrophages was observed in people carrying the A allele as compared with people carrying the GG genotype (+14.8\%; $p=0.026)$ (Figure 1A). For ABCG1 (rs2839482), when using a recessive model, it was found that people carrying the GG genotype had a higher plasma cholesterol efflux capacity $(+20.2 \%$; $p=0.044)$ compared with people carrying the A allele (Figure 1B). For LXR (rs2248949) a recessive model was used and a reduction in the plasma cholesterol efflux capacity $(-17.4 \% ; p \leq 0.001)$ was observed in people carrying the GG genotype compared with those carrying the A allele. Finally, for the locus rs2695121 of the LXR gene, a dominant model was used and an increased cholesterol efflux capacity $(+21.1 \%$; $p \leq 0.001)$ was observed in people carrying the A allele compared to those carrying the GG genotype (Figure 1C-1D). People carrying the GG genotype for CETP (rs1864163) showed an increased plasma cholesterol efflux capacity $(+14.7 \%$; $p \leq 0.01)$ compared to those carrying the A allele, when using a dominant model (Figure 1E).
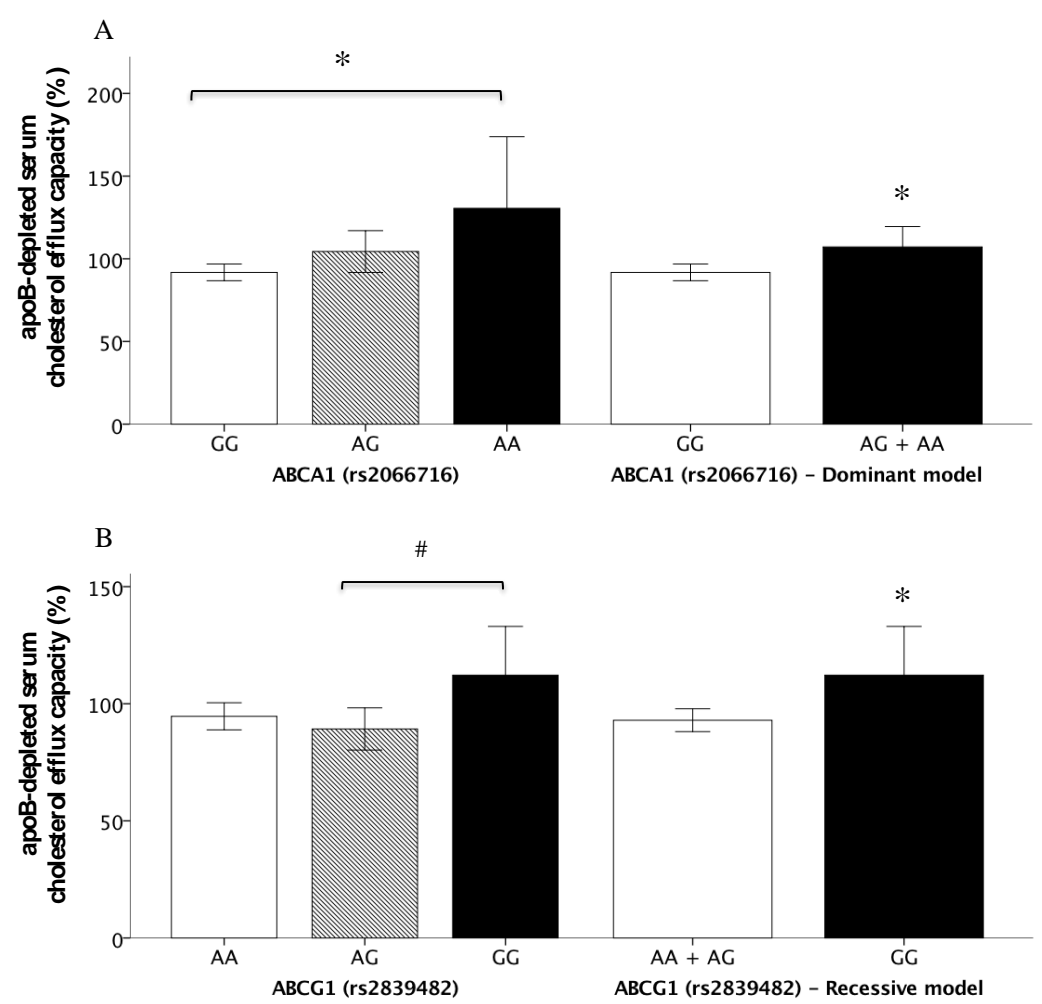

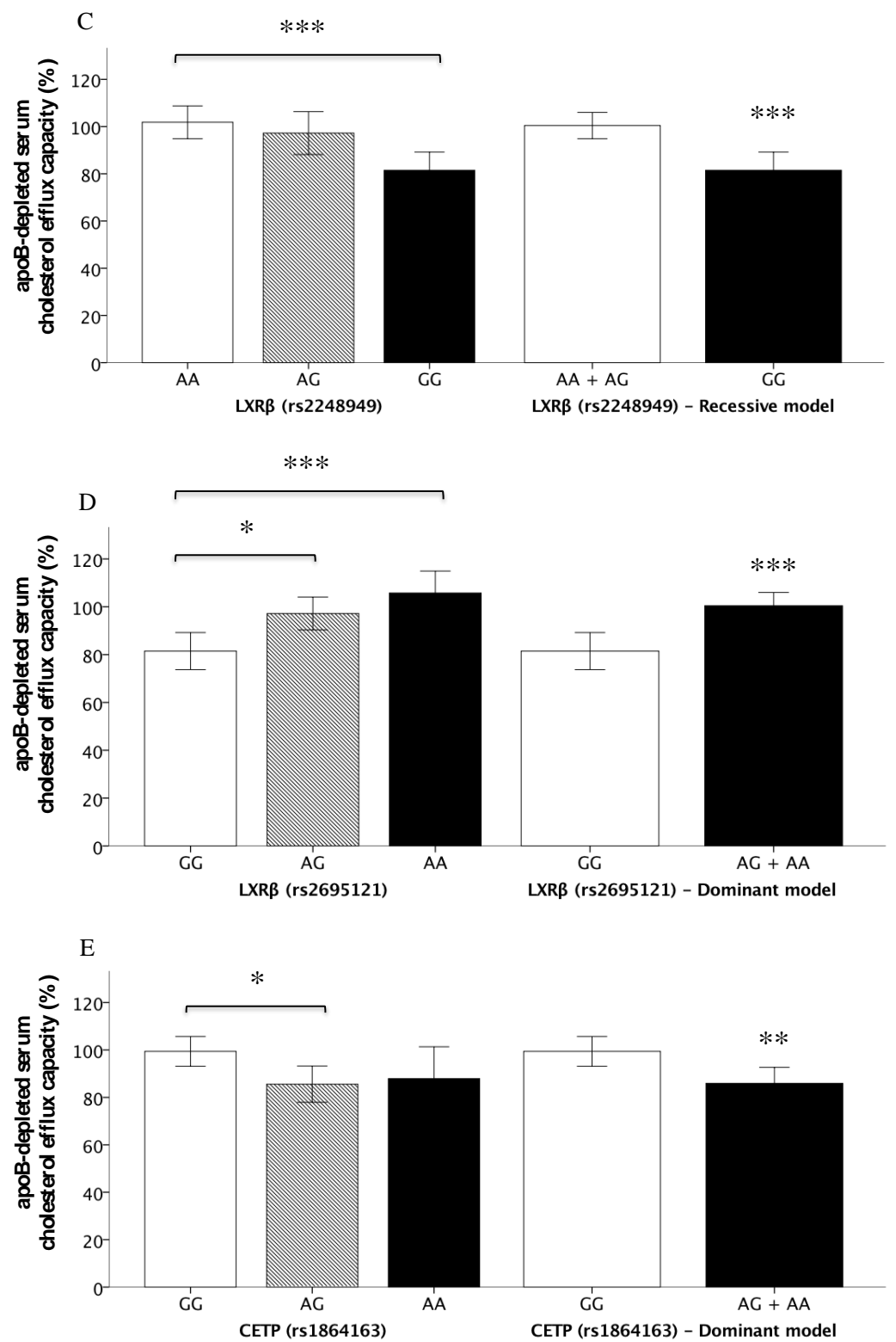

Figure 1: Association between selected SNPs with apoB-depleted serum cholesterol efflux capacity (\%), using dominant models $(A ; D ; E)$ or recessive models $(B ; C)$.

Values are means $\pm S E$. The black bars in the left panels refer to the least frequent homozygous genotype. $p$-values have been adjusted for the variables use of statins, age and HDL-C concentrations. ${ }^{*} p \leq 0.05,{ }^{* *} p \leq 0.01,{ }^{* * *} p \leq 0.001$, $\# p<0.08$. 


\section{Discussion}

We found that SNPs located in key genes involved in the RCT pathway (ABCA1, $A B C G 1, L X R \beta$ and CETP) were associated with the ability of HDL particles to promote cholesterol efflux from macrophages. In addition, significant positive correlations between cholesterol efflux capacity with HDL-C and apoA1 concentrations were found, which is in line with other studies [5, 22]. Also, cholesterol efflux capacity was significantly higher in women than in men. This difference in cholesterol efflux however could be explained by the higher HDL-C concentrations of the women, as the gender effect did not reach statistical significance anymore after adjustment for HDL-C concentrations.

Earlier studies have already reported that SNPs located in the ABCA1 and ABCG1 genes are related to HDL-C and apoA1 concentrations [23-25]. Also, mutations in the $A B C A 1$ gene have been associated with decreased HDL-C concentrations, a lower cholesterol efflux capacity, and an increased risk for CVD [26]. The importance of $A B C A 1$ and $A B C G 1$ in cholesterol efflux has further been demonstrated in studies with mice. When $A B C G 1$ was knocked down, cholesterol efflux was reduced and even further decreased when both $A B C G 1$ and $A B C A 1$ were knocked down [27]. These $A B C$-transporters assist in the transport of cholesterol from macrophages to HDL particles, but to different classes of HDL particles. $A B C A 1$ is involved in the transfer of cholesterol to lipid-poor apoA1, i.e. pre- $\beta$ HDL, while $A B C A 1$ and particularly $A B C G 1$ donate cholesterol to mature HDL, i.e. HDL2 and HDL3 [28-30]. No previous studies have examined associations between the SNP in ABCG1 (rs2839482) with cholesterol efflux capacity. For the SNP in ABCA1 (rs2066716), in one study a relation with HDL-C concentrations was observed, but not with cholesterol efflux [31]. However, results may be difficult to compare with ours as another method to measure cholesterol efflux was used. In their assay, efflux from macrophages cultured from isolated monocytes of each individual participant to purified human lipid-free apoA-I was measured. Also, only 43 men were studied. Villard et al., however, have reported in 450 men that another SNP in the $A B C A 1$ gene (rs2230806) was significantly related to plasma cholesterol efflux capacity [11]. In that study, men, but not women, carrying the minor allele had a $16.1 \%$ higher cholesterol efflux. In contrast, we did not find any association between this SNP with cholesterol efflux, which may relate to the lower number of subjects in our study compared with the study of Villard and colleagues.

LXR $\beta$ is involved in the activation of $A B C A 1$ and $A B C G 1$ [4, 32]. Minor alleles in the $L X R \beta$ gene (rs2695121 and rs2248949), i.e. the same as in our study, have been found to be associated with obesity [33] and impaired glucose metabolism [34]. We showed 
that people carrying the minor allele for rs2248949 had a lower capacity to promote cholesterol efflux from macrophages. As LXR $\beta$ plays a central role and is a key regulator of several metabolic pathways, these results together suggest that SNPs in the $L X R \beta$ gene may be related to a wide variety of different metabolic disorders.

Finally, we showed that people carrying the minor allele A for rs1864163, a SNP in the CETP gene, had a lower cholesterol efflux capacity. This allele is associated with lower HDL-C concentrations [35]. Subjects with CETP deficiency, who have higher HDL-C concentrations, also have an increased cholesterol efflux [27]. However, all our statistical analyses have been adjusted for HDL-C concentrations, meaning that differences in HDL-C concentrations between the subjects cannot explain our finding. Also, CETP activity may be positively associated with cholesterol efflux capacity from macrophages [36], and CETP protein may be associated with HDL particles [37], but we did not find any relations between CETP mass or activity with cholesterol efflux. Differences in cholesterol efflux therefore cannot be explained by differences in CETP activity or mass.

Differences in the number or composition of non-HDL particles between the samples of our subjects cannot explain the results, as the assay used was free of apoB-containing lipoproteins. It should also be realized that the proteins from the genes studied ( $A B C A 1, A B C G 1, L X R \beta$ and CETP) are not part of the HDL particles added to the murine J774 macrophages for the cholesterol efflux measurements. This raises the question if the SNPs that were studied are related to the composition and the size of the HDL particles as present in the plasma samples, as has also been shown for other SNPs [25, 38-40]. In fact, HDL particles size distribution is a determinant of the functionality of the total HDL fraction [30]. Both ABCA1 and ABCG1-mediated pathways contribute in cholesterol efflux to large HDL2 particles [30]. It is also known that women have more HDL2 particles than men [41], and that these larger HDL2 particles are related to an increased cholesterol efflux capacity via the SR-BI pathway in healthy women with increased HDL-C concentrations [42]. However, this seems to contradict studies suggesting that the major mediator of cholesterol efflux is small and dense pre- $\beta$ HDL particles [30, 43]. In addition, Villard et al. showed that genetic determinants relate to plasma cholesterol efflux capacity in a sex-specific manner [11], which might be due to gender differences in HDL particles size distribution. However, whether the SNPs studied in our study are related to HDL particle size distribution warrants further investigation.

In addition, all the SNPs studied were present within non-coding sections of the genes. It is therefore possible that SNPs were in linkage disequilibrium with other SNPs that have a functional impact on cholesterol efflux. Further, SNPs in noncoding regions can alter splicing of the mRNA and thereby modify the sequence in amino-acids and the function of the protein associated [44]. Moreover, introns may 
affect gene expression through a not well-understood mechanism called intronmediated enhancement (IME) [45]. Whatever the explanation, the proteins from these genes were not part of the assay, suggesting they have already modulated the functionality of the HDL particles in the circulation, before being sampled.

We found that the use of statins was related to a higher cholesterol efflux capacity. In one other study, rosuvastatin enhanced the capacity of HDL to promote cholesterol efflux via the ABCA1 pathway, without any changes in the HDL-C concentrations [46], but in other studies no effects of atorvastatin, pravastatin or simvastatin were found [5, 46, 47], [48]. It is also possible that different classes of statins impact differently cholesterol efflux capacity of HDL particles. However in our present study, the subjects that were on a current treatment with statins were not all using the same type of statins, although most of the subjects were using pravastatin [17].

Although the assay used to measure cholesterol efflux is inversely correlated with CVD [5], it predominantly quantifies one step of the RCT pathway, i.e. the ABCA1mediated cholesterol efflux, as its expression has been maximized with cAMP treatment. This assay also measures efflux via other parts of the RCT pathway, such as the SR-B1 and ABCG1-mediated pathways, and aqueous diffusion of free cholesterol, but to a lesser extent. However, it does not measure cholesterol excretion via its conversion into bile acids [49]. Our results do not therefore reflect the efficiency of the entire RCT pathway.

In conclusion, the present study demonstrates that cholesterol efflux capacity of the HDL fraction in plasma is related to SNPs located in genes encoding for $A B C A 1$, $A B C G 1, L X R \beta$ and CETP. Underlying mechanisms are unclear as these proteins are not all present in the HDL particles used in the cholesterol efflux assay. Although cholesterol efflux capacity is inversely associated with the incidence of CVD [50], it is also unknown if these specific SNPs are related to an increased risk to develop CVD, which warrants further investigation. 


\section{Supplementary data}

Table 1: Type of statin used by the subjects

\begin{tabular}{lcccc}
\hline \multicolumn{1}{c}{$\begin{array}{c}\text { Type of } \\
\text { statin }\end{array}$} & $\begin{array}{c}\text { Subjects } \\
\text { F/M }\end{array}$ & \multicolumn{3}{c}{$\begin{array}{c}\text { Cholesterol efflux } \\
\text { (\%) }\end{array}$} \\
\hline Simvastatin & $3 / 7$ & 90.1 & \pm & 11.7 \\
Pravastatin & $5 / 14$ & 94.7 & \pm & 31.8 \\
Atorvastatin & $5 / 5$ & 107.1 & \pm & $22.8^{*}$ \\
Rosuvastatin & $1 / 0$ & \multicolumn{4}{c}{102.6} \\
Fluvastatin & $1 / 0$ & \multicolumn{4}{c}{88.3} \\
None & $42 / 24$ & 92.7 & \pm & 24.7 \\
\hline
\end{tabular}

Values are means $\pm S D$.

F: Female; M: Male

p-values were adjusted for the variables age and HDL-C concentrations, after Bonferroni correction

Significantly different from the non-statin users: ${ }^{*} p \leq 0.05$ 
Chapter 3

Table 2: Functional consequences of the selected SNPs

\begin{tabular}{|c|c|c|}
\hline Genes & Loci names & Functional consequences \\
\hline ABCA1 & $\begin{array}{l}\text { rs2230806 } \\
\text { rs4149268 } \\
\text { rs1800977 } \\
\text { rs2066716 }\end{array}$ & $\begin{array}{l}\text { missense } \\
\text { intron variant } \\
\text { intron variant } \\
\text { synonymous codon }\end{array}$ \\
\hline ABCG1 & $\begin{array}{l}\text { rs915843 } \\
\text { rs2839482 }\end{array}$ & $\begin{array}{l}\text { intron variant } \\
\text { intron variant }\end{array}$ \\
\hline LXR $\alpha$ & $\begin{array}{l}\text { rs11039155 } \\
\text { rs2279238 }\end{array}$ & $\begin{array}{l}\text { intron variant } \\
\text { synonymous codon }\end{array}$ \\
\hline LXR $\beta$ & $\begin{array}{l}\text { rs2248949 } \\
\text { rs17373080 } \\
\text { rs1405655 } \\
\text { rs2695121 }\end{array}$ & $\begin{array}{l}\text { intron variant } \\
\text { upstream variant } 2 \mathrm{~KB} \\
\text { intron variant } \\
\text { intron variant }\end{array}$ \\
\hline SRB1 & rs5888 & synonymous codon \\
\hline CETP & $\begin{array}{l}\text { rs1566439 } \\
\text { rs1800777 } \\
\text { rs1864163 } \\
\text { rs12596776 } \\
\text { rs9989419 } \\
\text { rs5882 } \\
\text { rs1800775 } \\
\text { rs3764261 }\end{array}$ & $\begin{array}{l}\text { intron variant } \\
\text { missense } \\
\text { intron variant } \\
\text { intron variant } \\
\text { /no information } \\
\text { missense } \\
\text { upstream variant 2KB } \\
\text { /untested }\end{array}$ \\
\hline $\begin{array}{c}\text { Cluster } \\
\text { apoA5/A4/C3/A1 }\end{array}$ & $\begin{array}{l}\text { rs651821 } \\
\text { rs5128 } \\
\text { rs662799 } \\
\text { rs619054 } \\
\text { rs12286037 }\end{array}$ & $\begin{array}{l}\text { utr variant } 5^{\prime} \\
\text { utr variant } 3^{\prime} \\
\text { upstream variant 2KB } \\
\text { utr variant 3' } \\
\text { intron variant }\end{array}$ \\
\hline
\end{tabular}




\section{References}

1. Barter, P., et al., High density lipoproteins (HDLs) and atherosclerosis; the unanswered questions. Atherosclerosis, 2003. 168(2): p. 195-211.

2. Choi, B.G., et al., The role of high-density lipoprotein cholesterol in the prevention and possible treatment of cardiovascular diseases. Curr Mol Med, 2006. 6(5): p. 571-87.

3. Rader, D.J. and G.K. Hovingh, HDL and cardiovascular disease. Lancet, 2014. 384(9943): p. 618-25.

4. Tall, A.R., Cholesterol efflux pathways and other potential mechanisms involved in the athero-protective effect of high density lipoproteins. J Intern Med, 2008. 263(3): p. 256-73.

5. Khera, A.V., et al., Cholesterol efflux capacity, high-density lipoprotein function, and atherosclerosis. N Engl J Med, 2011. 364(2): p. 127-35.

6. Ritsch, A., H. Scharnagl, and W. Marz, HDL cholesterol efflux capacity and cardiovascular events. N Engl J Med, 2015. 372(19): p. 1870-1.

7. Wang, X. and B. Paigen, Genetics of variation in HDL cholesterol in humans and mice. Circ Res, 2005. 96(1): p. 27-42.

8. Chen, S.N., et al., Candidate genetic analysis of plasma high-density lipoprotein-cholesterol and severity of coronary atherosclerosis. BMC Med Genet, 2009. 10: p. 111.

9. Johannsen, T.H., et al., Genetic inhibition of CETP, ischemic vascular disease and mortality, and possible adverse effects. J Am Coll Cardiol, 2012. 60(20): p. 2041-8.

10. Thompson, A., et al., Association of cholesteryl ester transfer protein genotypes with CETP mass and activity, lipid levels, and coronary risk. Jama, 2008. 299(23): p. 2777-88.

11. Villard, E.F., et al., Genetic determination of plasma cholesterol efflux capacity is genderspecific and independent of HDL-cholesterol levels. Arterioscler Thromb Vasc Biol, 2013. 33(4): p. 822-8.

12. Berrougui, H., et al., Age-related impairment of HDL-mediated cholesterol efflux. J Lipid Res, 2007. 48(2): p. 328-36.

13. Gardner, C.D., et al., Population frequency distributions of $H D L, H D L(2)$, and $H D L(3)$ cholesterol and apolipoproteins A-I and B in healthy men and women and associations with age, gender, hormonal status, and sex hormone use: the Stanford Five City Project. Prev Med, 2000. 31(4): p. 335-45.

14. Nieves, D.J., et al., The atherogenic lipoprotein profile associated with obesity and insulin resistance is largely attributable to intra-abdominal fat. Diabetes, 2003. 52(1): p. 172-9.

15. McTaggart, F. and P. Jones, Effects of Statins on High-Density Lipoproteins: A Potential Contribution to Cardiovascular Benefit. Cardiovasc Drugs Ther, 2008. 22(4): p. 321-38.

16. Plat, J. and R.P. Mensink, Vegetable oil based versus wood based stanol ester mixtures effects on serum lipids and hemostatic factors in non-hypercholesterolemic subjects. Atherosclerosis, 2000. 148(1): p. 101-12.

17. de Jong, A., et al., Effects of long-term plant sterol or stanol ester consumption on lipid and lipoprotein metabolism in subjects on statin treatment. Br J Nutr, 2008. 100(5): p. 937-41.

18. Scharnagl, H., et al., Decreased cholesterol efflux capacity in patients with low cholesteryl ester transfer protein plasma levels. Eur J Clin Invest, 2014. 44(4): p. 395-401. 
19. Ritsch, A., et al., Phage-displayed recombinant single-chain antibody fragments with high affinity for cholesteryl ester transfer protein (CETP): cDNA cloning, characterization and CETP quantification. Clin Chem Lab Med, 2004. 42(3): p. 247-55.

20. Ritsch, A., et al., Enhancement of cholesteryl ester transfer in plasma by hormonereplacement therapy. Metabolism, 2002. 51(5): p. 599-604.

21. Kaser, S., et al., Lipoprotein profile and cholesteryl ester transfer protein in neonates. Metabolism, 2001. 50(6): p. 723-8.

22. Saleheen, D., et al., Association of HDL cholesterol efflux capacity with incident coronary heart disease events: a prospective case-control study. Lancet Diabetes Endocrinol, 2015. 3(7): p. 507-13.

23. Frikke-Schmidt, R., et al., Genetic variation in ABC transporter A1 contributes to HDL cholesterol in the general population. J Clin Invest, 2004. 114(9): p. 1343-53.

24. Porchay, I., et al., ABCA1 single nucleotide polymorphisms on high-density lipoproteincholesterol and overweight: the D.E.S.I.R. study. Obesity (Silver Spring), 2006. 14(11): p. 1874-9.

25. Zago, V.H., et al., Association between ABCG1 polymorphism rs1893590 and high-density lipoprotein (HDL) in an asymptomatic Brazilian population. Mol Biol Rep, 2015. 42(3): p. 745-54.

26. Bochem, A.E., et al., ABCA1 mutation carriers with low high-density lipoprotein cholesterol are characterized by a larger atherosclerotic burden. Eur Heart J, 2013. 34(4): p. 286-91.

27. Tall, A.R., The effects of cholesterol ester transfer protein inhibition on cholesterol efflux. Am J Cardiol, 2009. 104(10 Suppl): p. 39e-45e.

28. Yvan-Charvet, L., N. Wang, and A.R. Tall, Role of HDL, ABCA1, and ABCG1 transporters in cholesterol efflux and immune responses. Arterioscler Thromb Vasc Biol, 2010. 30(2): p. 139-43.

29. Tavoosi, Z., et al., Cholesterol Transporters ABCA1 and ABCG1 Gene Expression in Peripheral Blood Mononuclear Cells in Patients with Metabolic Syndrome. Cholesterol, 2015. 2015: p. 682904.

30. Du, X.M., et al., HDL particle size is a critical determinant of ABCA1-mediated macrophage cellular cholesterol export. Circ Res, 2015. 116(7): p. 1133-42.

31. Soro-Paavonen, A., et al., Common ABCA1 variants, HDL levels, and cellular cholesterol efflux in subjects with familial low HDL. J Lipid Res, 2007. 48(6): p. 1409-16.

32. Lund, E.G., et al., Different roles of liver X receptor alpha and beta in lipid metabolism: effects of an alpha-selective and a dual agonist in mice deficient in each subtype. Biochem Pharmacol, 2006. 71(4): p. 453-63.

33. Dahlman, I., et al., Liver $X$ receptor gene polymorphisms and adipose tissue expression levels in obesity. Pharmacogenet Genomics, 2006. 16(12): p. 881-9.

34. Ketterer, C., et al., Genetic variation within the NR1H2 gene encoding liver X receptor beta associates with insulin secretion in subjects at increased risk for type 2 diabetes. J Mol Med (Berl), 2011. 89(1): p. 75-81.

35. Dumitrescu, L., et al., Genetic determinants of lipid traits in diverse populations from the population architecture using genomics and epidemiology (PAGE) study. PLoS Genet, 2011. 7(6): p. e1002138. 
36. Villard, E.F., et al., Elevated CETP activity improves plasma cholesterol efflux capacity from human macrophages in women. Arterioscler Thromb Vasc Biol, 2012. 32(10): p. 2341-9.

37. Lee-Rueckert, M., et al., Association of cholesteryl ester transfer protein with HDL particles reduces its proteolytic inactivation by mast cell chymase. J Lipid Res, 2008. 49(2): p. 35868.

38. Frazier-Wood, A.C., et al., Genetic variants associated with VLDL, LDL and HDL particle size differ with racelethnicity. Hum Genet, 2013. 132(4): p. 405-13.

39. Ruel, I.L., et al., Determinants of HDL particle size in patients with the null (P207L) or defective (D9N) mutation in the lipoprotein lipase gene: the Quebec LipD Study. Atherosclerosis, 2002. 162(2): p. 269-76.

40. Tsuzaki, K., et al., The relationship between adiponectin, an adiponectin gene polymorphism, and high-density lipoprotein particle size: from the Mima study. Metabolism, 2012. 61(1): p. 17-21.

41. Pascot, A., et al., HDL particle size: a marker of the gender difference in the metabolic risk profile. Atherosclerosis, 2002. 160(2): p. 399-406.

42. Catalano, G., et al., Cellular SR-BI and ABCA1-mediated cholesterol efflux are genderspecific in healthy subjects. J Lipid Res, 2008. 49(3): p. 635-43.

43. Heinecke, J.W., Small HDL promotes cholesterol efflux by the ABCA1 pathway in macrophages: implications for therapies targeted to HDL. Circ Res, 2015. 116(7): p. 11013.

44. Wang, G.S. and T.A. Cooper, Splicing in disease: disruption of the splicing code and the decoding machinery. Nat Rev Genet, 2007. 8(10): p. 749-61.

45. Gallegos, J.E. and A.B. Rose, The enduring mystery of intron-mediated enhancement. Plant Sci, 2015. 237: p. 8-15.

46. Shimizu, T., et al., Rosuvastatin activates ATP-binding cassette transporter A1-dependent efflux ex vivo and promotes reverse cholesterol transport in macrophage cells in mice fed a high-fat diet. Arterioscler Thromb Vasc Biol, 2014. 34(10): p. 2246-53.

47. Ronsein, G.E., et al., Niacin Therapy Increases High-Density Lipoprotein Particles and Total Cholesterol Efflux Capacity But Not ABCA1-Specific Cholesterol Efflux in StatinTreated Subjects. Arterioscler Thromb Vasc Biol, 2016. 36(2): p. 404-11.

48. de Vries, R., et al., Cellular cholesterol efflux to plasma from moderately hypercholesterolaemic type 1 diabetic patients is enhanced, and is unaffected by simvastatin treatment. Diabetologia, 2005. 48(6): p. 1105-13.

49. Yancey, P.G., et al., Importance of different pathways of cellular cholesterol efflux. Arterioscler Thromb Vasc Biol, 2003. 23(5): p. 712-9.

50. Rohatgi, A., et al., HDL cholesterol efflux capacity and incident cardiovascular events. N Engl J Med, 2014. 371(25): p. 2383-93. 


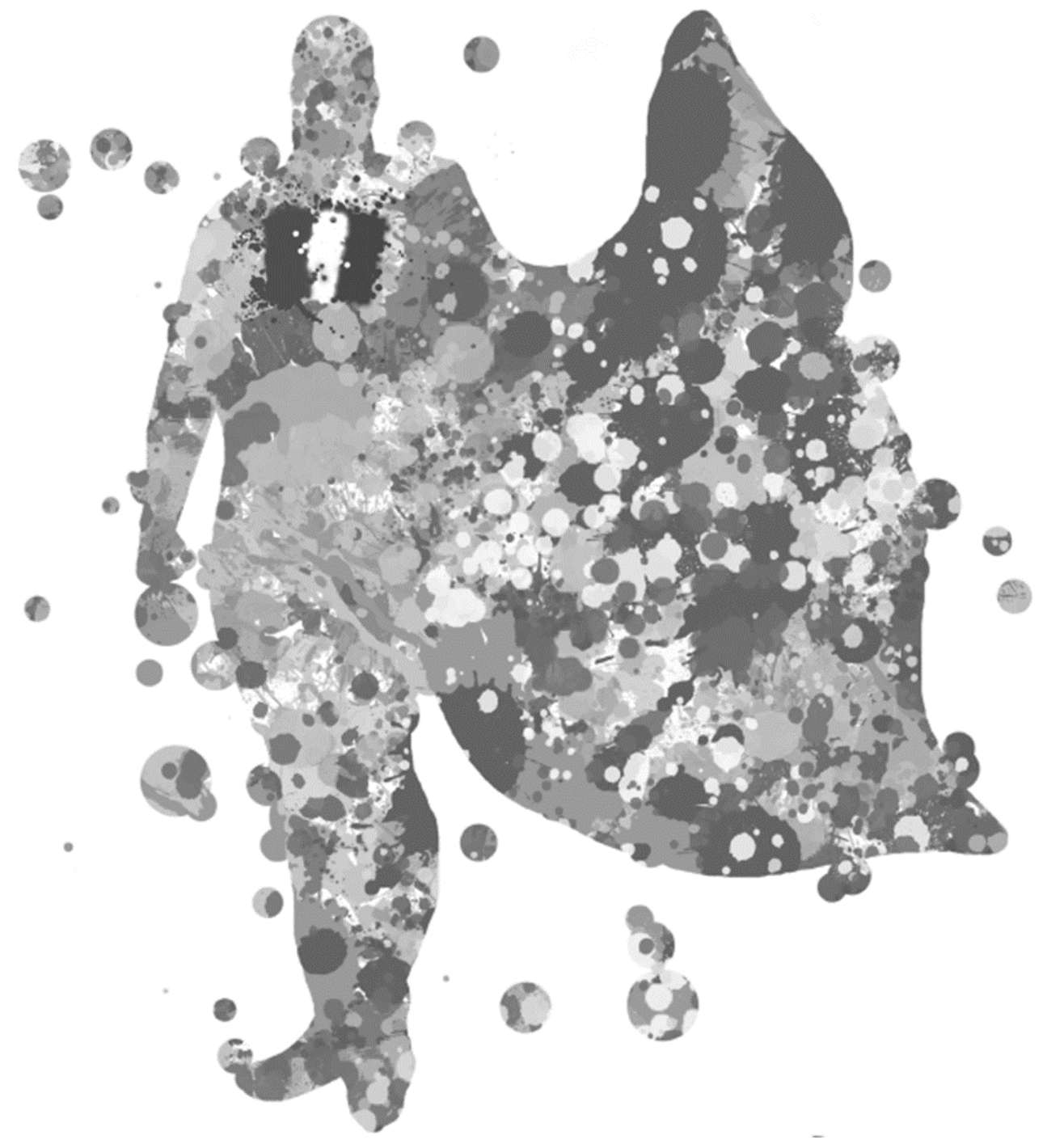




\section{Chapter 4}

\section{Theobromine does not affect}

fasting and postprandial HDL cholesterol efflux capacity, while it decreases fasting miR-92a

\section{levels in humans}

Charlotte P.J. Talbot, Ronald P. Mensink, Lotte Smolders, Virginie Bakeroot, Jogchum Plat

Submitted

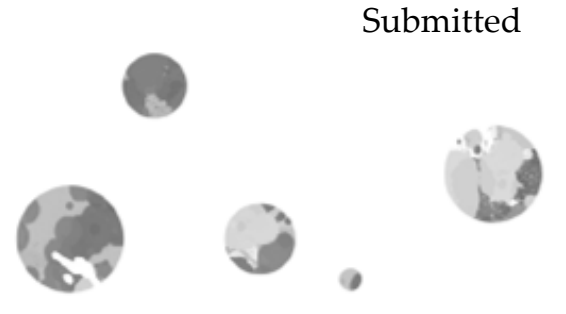




\section{Abstract}

Background and Aims: MicroRNAs (miRNAs) can be incorporated into highdensity lipoproteins (HDL) particles, transmitted to recipient cells and as such, might facilitate HDL functionality. However, not much is known about the effects of diet on miRNAs concentrations and HDL-mediated cholesterol efflux. Chocolate consumption is associated with a reduction in the risk to develop cardiovascular disease (CVD), which might be attributed to theobromine, a methylxanthine found in cocoa. Therefore, the aim of this study was to investigate the effects of long-term theobromine consumption on fasting and postprandial HDL-mediated cholesterol efflux and on miRNAs levels in apolipoprotein B (apoB)-depleted serum, in overweight and obese men and women.

Methods: Thirty overweight and 14 obese healthy subjects were included in this randomized, double-blind crossover study. Participants had to consume $500 \mathrm{mg}$ of theobromine or placebo daily for 4 weeks. At the end of the intervention, fasting and postprandial samples were collected. Cholesterol efflux was measured in BODIPYlabeled murine J774 macrophages with cAMP induced ABCA1 transporter activation, and incubation with apolipoprotein B-depleted serum samples of the subjects. Three miRNAs known to be associated with HDL particles were selected (miR-92a; miR-223 and miR-135a*), and their levels in apoB-depleted serum were quantified by TaqMan MicroRNA Assays.

Results: 4 weeks theobromine consumption did not affect fasting and postprandial HDL cholesterol efflux. Fasting miR-223 and miR-135a levels were unchanged, while miR-92a levels were decreased after the intervention $(-0.21 ; p<0.05)$. High fat meal intake increased postprandial cholesterol efflux capacity by 4.3 percentage point $(\mathrm{p} \leq 0.001)$, as well as miR-92a $(+1.21 ; \mathrm{p}<0.001)$ and $\operatorname{miR}-223(+1.79 ; \mathrm{p}<0.001)$ levels, while a trend was found for miR-135a levels $(+1.08 ; \mathrm{p}=0.06)$.

Conclusions: Theobromine consumption did not improve cholesterol efflux capacity at baseline and postprandially, while it decreased fasting levels of miR-92a. In addition, high fat meal intake increased postprandial cholesterol efflux and levels of the three selected miRNAs. 


\section{Introduction}

MicroRNAs (miRNA) are small, non-coding RNAs composed of about 22 nucleotides that bind to complementary sites in the $3^{\prime} \mathrm{UTR}$ region of their target RNAs, thereby inhibiting gene expression through post-transcriptional regulation [1]. MiRNAs can be encapsulated within vesicles, such as exosomes, which are subsequently released into the circulation. However, miRNAs can also bind to protein complexes or be associated with lipoproteins [2]. Vickers et al. were the first to demonstrate that high-density lipoprotein (HDL) particles isolated from human plasma contain miRNAs, which can subsequently be transmitted in vitro to hepatocytes cells via scavenger receptor BI (SR-BI) [3]. In addition, HDL miR-223 can be transferred from HDL particles to human coronary artery endothelial cells, also through SR-BI [4]. MiRNAs are also transported by other lipoproteins, but in smaller amounts [5].

In humans, the HDL-miRNAs signature is different under cardiovascular and hypercholesterolemic conditions, although the specific roles of the different miRNAs are largely unknown [3]. Among the many miRNAs, miR-223, miR-135a and miR-92a belong to the most abundant HDL-associated miRNAs [3, 5, 6]. MiR223 has previously been reported to indirectly increase cholesterol efflux, the main anti-atherogenic effect of HDL [7]. Whether miR-135a and miR-92a are also related to cholesterol efflux has never been studied.

Not much is known about the effects of diet on HDL-mediated cholesterol efflux and miRNAs concentrations, which may provide explanations for observed associations between diet or dietary components with CVD-risk. Chocolate consumption, for example, is associated with a reduction in the risk to develop cardiovascular disease (CVD) [8]. These effects may be mediated through effects on flow-mediated vasodilation, which has been reported to increase after dark chocolate intake [9]. Also, HDL-C concentrations may increase, although results are controversial [10-12]. However, it is questionable whether increasing HDL-C concentrations protects against CVD [13]. Further, it has been suggested that only dark chocolate have cardio-protective effects. Dark chocolate is rich in methylxanthines, including theobromine [14], which may increase HDL-C and apoA-I concentrations, the main apolipoprotein within HDL [15]. However, effects of theobromine consumption on fasting and postprandial HDL-mediated cholesterol efflux capacity have never been investigated. In fact, not much is known at all about changes in cholesterol efflux during the postprandial phase, although disturbances in postprandial lipoprotein metabolism are an important risk factor for CVD [16, 17]. Therefore, the effect of theobromine intake on HDL-mediated cholesterol efflux capacity was investigated in 44 overweight and obese men and women. In addition, changes in miRNAs levels in apolipoprotein B (apoB)-depleted serum (e.g. miR-223, miR-135a, and miR-92a) 
following theobromine and meal consumption were examined. Finally, relationships between changes in the expression of these miRNAs and variations in cholesterol efflux capacity as well as serum lipids were assessed.

\section{Materials and Methods}

\section{Subjects}

Thirty overweight (body mass index, BMI, $\mathrm{kg} / \mathrm{m}^{2}$ : 25-30) and 14 obese (BMI: 30-35) apparently healthy subjects participated in this study, which examined the effects of theobromine consumption on cardiometabolic health. The 28 men were aged between $45-70$ years $(60.2 \pm 5.2$ years), with a BMI of $28.6 \pm 2.7$. Six of the men were obese. The 16 women were aged between $50-70$ years (60.5 \pm 6.0 years) with a BMI of $30.2 \pm 3.2$. Eight of the women were obese. All subjects had low baseline HDL-C concentrations $(<1.2 \mathrm{mmol} / \mathrm{L}$ for men and $<1.5 \mathrm{mmol} / \mathrm{L}$ for women) and were not using any medications. Full details of the inclusion criteria have been published before [18]. The study was conducted according the ethical guidelines of the 1975 Declaration of Helsinki and approved by the Ethics Committee of Maastricht University Medical Centre. Written informed consent was obtained from all participants before entering the study. The study was registered at clinicaltrials.gov as NCT02209025.

\section{Studies design}

This study had a randomized, double blinded placebo-controlled, crossover design, including 2 intervention periods of 4-weeks, which were separated by a 4-week washout period. Two weeks before the start of the study and during the study, subjects had to avoid the use of products containing cocoa, as well as to limit their consumption of caffeine-containing drinks, as theobromine is a metabolite of caffeine. Subjects were randomly allocated to a group starting with a theobromine or placebo drink. During the 4 weeks intervention periods, subjects had to consume daily a $20 \mathrm{ml}$ drink enriched with either $500 \mathrm{mg}$ theobromine or placebo. At the end of both 4 weeks intervention periods (day 28), subjects participated in a postprandial test, for which they had to consume a shake $(460 \mathrm{ml})$ that provided $965 \mathrm{kcal}(17.9 \mathrm{~g}$ proteins, $85.7 \mathrm{~g}$ carbohydrates and $60.6 \mathrm{~g}$ fat), which had to be consumed within 10 minutes together with their test drink (Supplemental Table 1). 


\section{Blood sampling}

At day 28 of both the intervention and the placebo period, an intravenous cannula was inserted into a forearm vein and fasting blood samples were collected before the intake of the shake $(\mathrm{T}=0)$. Postprandial blood samples were sampled 2 hours $(\mathrm{T}=120)$ following shake consumption. Blood was collected in a $4 \mathrm{~mL}$ EDTA tube and then centrifuged at $1300 \mathrm{xg}$ for 15 minutes at $4^{\circ} \mathrm{C}$ to obtain plasma. To obtain serum, samples were allowed to clot for 1 hour at $20^{\circ} \mathrm{C}$, and then centrifuged at $1300 \times \mathrm{g}$ for $15 \mathrm{~min}$ at $20^{\circ} \mathrm{C}$. Plasma and serum supernatants were transferred into $1.5 \mathrm{ml}$ Eppendorf tubes and stored at $-80^{\circ} \mathrm{C}$ until use.

\section{Biochemical Measurements}

In all fasting $(\mathrm{T}=0)$ and postprandial $(\mathrm{T}=120)$ serum samples obtained at day 28 of both periods, HDL-C concentrations (precipitation of apoB-containing lipoproteins; Roche Diagnostics System, Hofmann-La Roche Ltd., Basel, Swiss), apoA1 (immunoturbidimetric reaction; Horiba $\mathrm{ABX}$, Montpellier Cedex, France) and triacylglycerol (TAG) (TRIGL: ACN 8781; Roche, Mannheim, Germany) concentrations were determined. All samples from one subject were analyzed within the same run. As only values at day 28 were used in the present study, results may therefore slightly differ from the original study, which reported the average values [18].

The ability of the HDL particles to promote cholesterol efflux from macrophages was measured as previously described [19]. Briefly, murine J774 macrophages were cultivated and incubated overnight with BODIPY-cholesterol. Afterwards, macrophages were equilibrated with an ACAT inhibitor (Acyl CoA Acyltransferase Inhibitor; Sandoz 58-035) to block cholesterol esterification, and the cholesterolefflux transporter ABCA1 was upregulated with cAMP (cyclic adenosine monophosphate; C3912, Sigma). To obtain HDL, human serum samples were depleted of apolipoprotein-B containing lipoproteins using tungstophosphoric acid hydrate and magnesium chloride, and the isolated HDL particles were then incubated with the labeled macrophages. All steps were performed in the presence of $1 \mu \mathrm{l} / \mathrm{ml}$ of ACAT inhibitor. For normalization, serum samples from two healthy subjects were included in each assay. The efflux capacity values obtained for each subject were normalized to the average efflux capacity value of these two pooled serum, which was set to $100 \%$ (\% pools). All samples were analyzed in triplicate. 


\section{MicroRNAs analysis}

\section{HDL isolation}

HDL particles were isolated from plasma samples by an apoB-containing lipoprotein depletion method, using polyethylene glycol (PEG, molecular weight (MW) 6,000; Sigma-Aldrich, St. Louis, MO), as previously described [20]. Briefly, 200 $\mu \mathrm{l}$ PEG $(200 \mathrm{mg} / \mathrm{ml})$ was added to $500 \mu \mathrm{l}$ of plasma. Samples were incubated for 20 $\mathrm{min}$ at room temperature, and centrifuged at $10000 \mathrm{rpm}$ for $30 \mathrm{~min}$. Supernatants, containing the HDL particles, were collected in $1.5 \mathrm{~mL}$ Eppendorf tubes and stored at $-80^{\circ} \mathrm{C}$ until use.

\section{Total RNA isolation}

Total RNA was isolated from $150 \mu$ isolated HDL, using the miRVana PARIS kit (Ambion, Austin, TX, USA). Samples were eluted with $50 \mu$ pre-heated at $95{ }^{\circ} \mathrm{C}$ elution solution. For normalization of miR expression, samples were spiked with 1.6 $\mu \mathrm{l}$ of $5 \mathrm{nM}$ of Caenorhabditis elegans (cel) miR-39 (cel-miR-39, RNA oligonucleotide, Sigma-Aldrich) after the addition of the denaturing solution. Cel-miR-39 was used as external control because of the absence of homologous sequences in humans [21].

\section{MicroRNA individual TaqMan assays}

MicroRNAs known to be present in HDL particles and described to be altered in cardiometabolic diseases were chosen, i.e. miR-135a*, miR-223-3p and miR-92a [3, 5, 6]. The primer sequences used are shown in Table 1.

Table 1: miRNAs primer sequences

\begin{tabular}{ll}
\hline \multicolumn{1}{c}{ miRNA } & \multicolumn{1}{c}{ Primers sequence } \\
\hline hsa-miR-135a* (3p) [3] & 5' UAUAGGGAUUGGAGCCGUGGCG 3' \\
hsa-miR-223-3p [3] & 5' UGUCAGUUUGUCAAAUACCCCA 3’ \\
hsa-miR-92a [6] & 5' UAUUGCACUUGUCCCGGCCUGU 3' \\
cel-miR-39 [53] & 5' UCACCGGGUGUAAAUCAGCUUG 33' \\
\hline
\end{tabular}

Total RNA was reverse transcribed using the TaqMan MicroRNA Reverse Transcription Kit (Applied Biosystems, Thermo Fisher Scientific, USA) in a total reaction volume of $15 \mu \mathrm{l}$. Briefly, $6 \mu \mathrm{l}$ of the total RNA including the external control $(5 \mathrm{nM})$ was used in addition with $3 \mu \mathrm{l}$ of the specific miRNAs primer (5X) and $6 \mu \mathrm{l}$ of the Mix solution (i.e. $1.5 \mu \mathrm{l}$ 10X RT buffer, $0.15 \mu \mathrm{l}$ dNTPs, $0.19 \mu \mathrm{l}$ RNAse inhibitor, 
$1 \mu \mathrm{l}$ Multiscribe RT enzyme and $3.16 \mu \mathrm{l}$ water). The reaction mixtures were incubated at $16^{\circ} \mathrm{C}$ for $30 \mathrm{~min}$, followed by $30 \mathrm{~min}$ at $42^{\circ} \mathrm{C}$ and $5 \mathrm{~min}$ at $85^{\circ} \mathrm{C}$, and then held at $4{ }^{\circ} \mathrm{C}$.

Subsequently, individual TaqMan MicroRNA Assays (Applied Biosystems, USA) were used for detection of miRNAs expression by real-time PCR. For this, $3 \mu \mathrm{l}$ of the reverse transcription product was mixed with $10 \mu \mathrm{l}$ of the Taqman Universal PCR Master Mix II (2X) no AmpErase UNG (Applied Biosystems, USA), together with 1 $\mu \mathrm{l}$ of specific miRNAs primer (20X) and water to a final reaction volume of $20 \mu \mathrm{l}$. Real-time PCR was performed in duplicate on a 7300 real-time PCR system (Applied Biosystems, USA), and the reaction mixtures were incubated at $95{ }^{\circ} \mathrm{C}$ for $10 \mathrm{~min}$, followed by 40 cycles of $95^{\circ} \mathrm{C}$ for $15 \mathrm{~s}$ and $60^{\circ} \mathrm{C}$ for $1 \mathrm{~min}$. The cycle threshold (CT) values were calculated with SDS v1.4 software using automatic threshold (Applied Biosystems, USA). The expression levels of the target miRNAs in apoB-depleted serum were normalized relative to the expression of cel-miR-39, and were calculated using the ${ }^{-}-\triangle \triangle C T$ method in order to determine the fold change in gene expression [22].

\section{Statistics}

A paired-samples t-test was used to examine the effects of theobromine on fasting parameters. Postprandial changes were calculated as the difference between 2 hours $(T=120)$ after the intake of the mixed meal and fasting measurements $(T=0)$, and were analyzed during the placebo and theobromine period separately with a pairedsamples t-test. Differences in postprandial changes between the two periods were also analyzed with a paired-samples t-test.

For the miRNAs analysis, Grubbs' test was used for the detection of outliers that were not physiologically plausible, which were removed from analysis. To examine the postprandial and diet effects on miRNAs levels, a one-samples t-test was used, with the baseline test value ( $\mathrm{T}=0$ or Placebo) set to 1 . Differences in changes in miRNAs levels between the two periods were performed with a paired-samples ttest. Correlations between fold changes of miRNA levels with age, as well as with changes in biochemical parameters (cholesterol efflux capacity and concentrations of HDL-C, apoA-I and TAG) after the high-fat test meal intake were performed using bivariate Pearson correlation coefficients.

A $P$ value $\leq 0.05$ was considered significant. All statistical analyses were performed with SPSS 23.0 (IBM, Armonk, NY) for Mac OS. 


\section{Results}

Effects of theobromine on fasting and postprandial cholesterol efflux, serum lipids and lipoproteins

Four weeks theobromine supplementation did not affect fasting cholesterol efflux capacity ( +0.4 percentage point (pp); $95 \% \mathrm{CI}:-2.81,3.57$; $\mathrm{p}=0.81$ ), but significantly increased HDL-C concentrations $(+0.04 \mathrm{mmol} / \mathrm{L} ; 95 \% \mathrm{CI}: 0.003,0.07 ; \mathrm{p}<0.05)$. Furthermore, apoA1 ( $+0.01 \mathrm{~g} / \mathrm{L} ; 95 \% \mathrm{CI}:-0.02,0.05 ; \mathrm{p}=0.50)$ and TAG concentrations ( $0.15 \mathrm{mmol} / \mathrm{L} ; 95 \% \mathrm{CI}:-0.03,0.33 ; \mathrm{p}=0.10)$ did not change.

During the control period, the intake of the test meal significantly increased cholesterol efflux after 2 hours ( +4.3 pp; 95\% CI: 1.87, 6.68; p $\leq 0.001)$. However, during the theobromine period no postprandial increase was observed (+1.6 pp; $95 \%$ CI: $-1.25,4.42 ; \mathrm{p}=0.26$ ). The test meal did not affect HDL-C concentrations in both conditions, i.e. without $(+0.01 \mathrm{mmol} / \mathrm{L} ; 95 \% \mathrm{CI}:-0.01,0.03 ; \mathrm{p}=0.16)$ or with theobromine; ( $+0.003 \mathrm{mmol} / \mathrm{L} ; 95 \% \mathrm{CI}:-0.01,0.02 ; \mathrm{p}=0.69)$, During the control period, apoA1 concentrations $(+0.02 \mathrm{~g} / \mathrm{L} ; 95 \%$ CI: $0.004,0.05 ; \mathrm{p}<0.05)$ and $\mathrm{TAG}$ concentrations $(+1.23 \mathrm{mmol} / \mathrm{L} ; 95 \% \mathrm{CI}:-1.04,1.43 ; \mathrm{p} \leq 0.001)$ significantly increased after test meal consumption. Comparable results were found during the theobromine period (apoA1: $+0.03 \mathrm{~g} / \mathrm{L} ; 95 \%$ CI: $0.01,0.04 ; \mathrm{p}<0.001$; TAG: +1.23 $\mathrm{mmol} / \mathrm{L} ; 95 \%$ CI: 1.03, 1.42; $\mathrm{p}<0.001)$.

Postprandial changes were similar between both periods for cholesterol efflux (-2.7 pp; 95\% CI: -.657, 1.19; $\mathrm{p}=0.17)$, as well as for the concentrations of HDL-C $(-0.01$ mmol/L; 95\% CI: $-0.04,0.02 ; \mathrm{p}=0.47)$, apoA1 (0.002 g/L; 95\% CI: $-0.02,0.03 ; \mathrm{p}=0.85)$ and TAG (0.02 mmol/L; 95\% CI: -0.15, 0.18; $\mathrm{p}=0.85)$ (Table 2). 


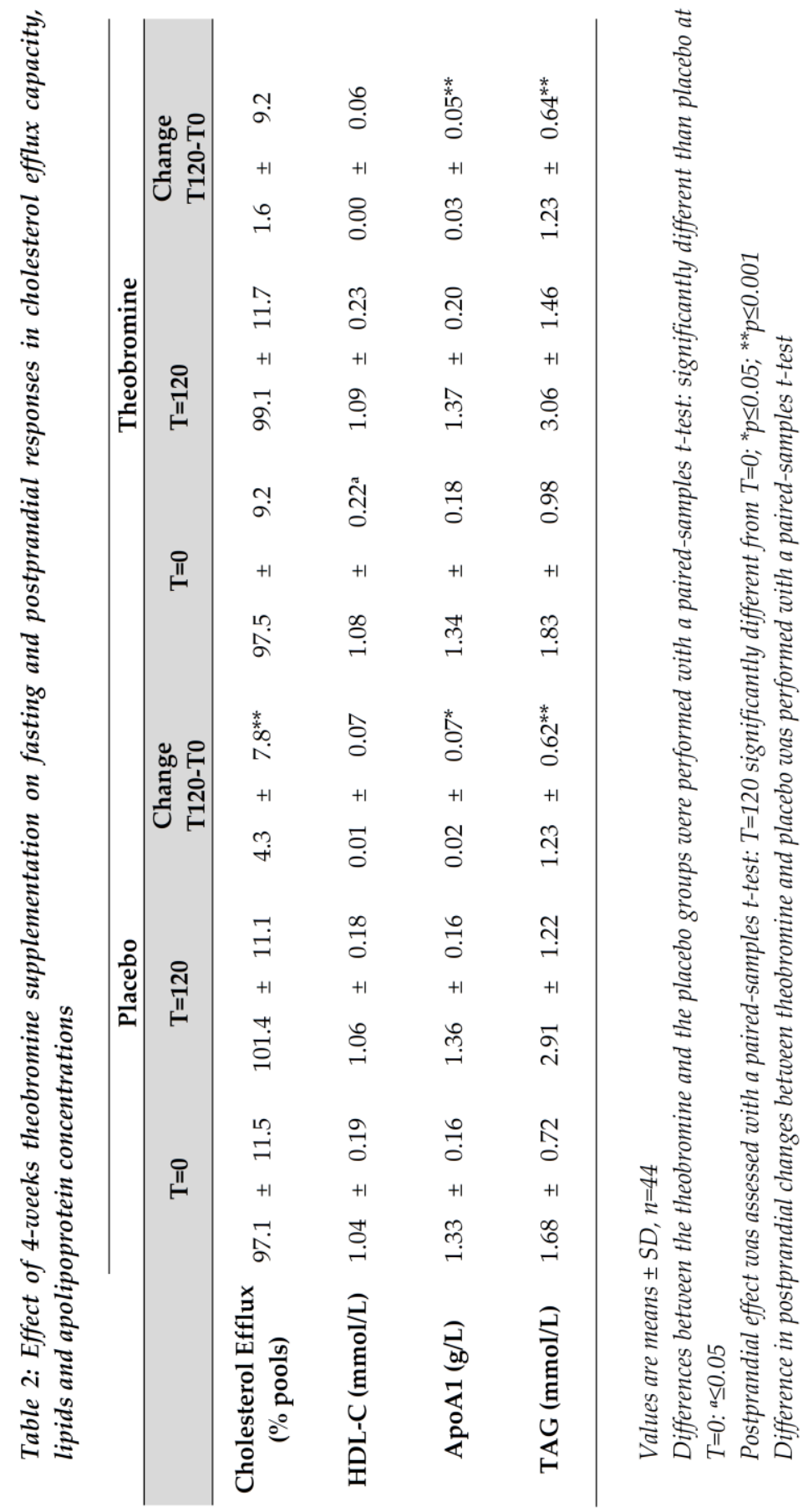




\section{Effect of theobromine on fasting and postprandial miRNAs levels}

Using the Grubbs' test for normality, 17 out of the 396 miRNA measurements carried out in this study were removed from the analysis. For the fasting results, 9 values were omitted. For the postprandial results, 5 values were removed in the placebo period, while 3 values were removed in the theobromine period.

Theobromine supplementation for 4 weeks reduced fasting miR-92a levels $(-0.21$; 95\% CI: $-0.38,-0.03 ; \mathrm{p}<0.05)$, while no effects were found on miR-223 $(+0.09 ; 95 \% \mathrm{CI}$ : $-0.22,0.41 ; p=0.55)$ and miR-135a levels $(-0.12 ; 95 \%$ CI: $-0.35,0.10 ; p=0.26$; Figure 1).

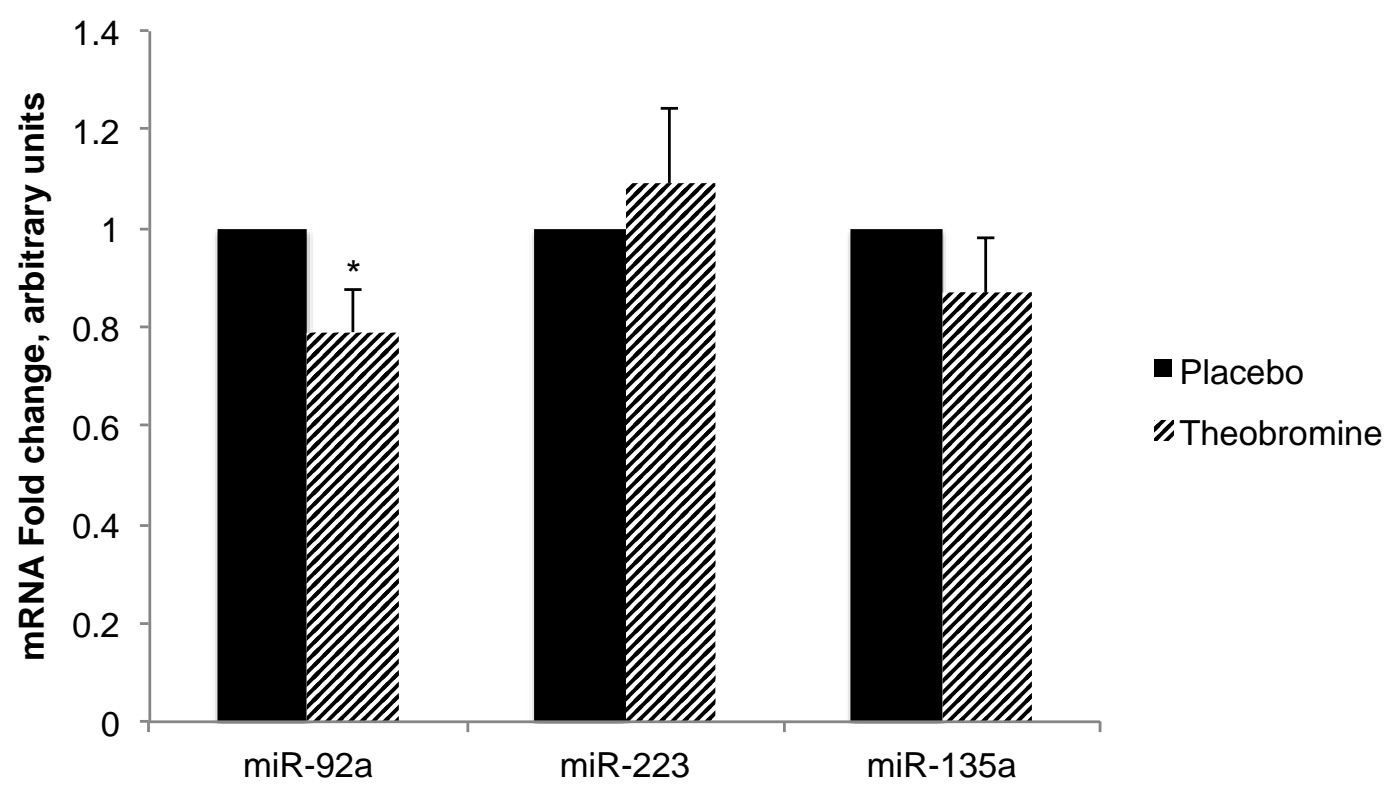

Figure 1: Fold changes of fasting miRNAs levels after 4 weeks of theobromine supplementation.

Fasting gene expression after 4 weeks of theobromine consumption relative to fasting gene expression after 4 weeks of placebo consumption.

Difference between theobromine and placebo (diet effect at T0) was performed with a one-samples t-test, with the test value set to $1:{ }^{*} p \leq 0.05$

miR-92a: $n=40$

miR-223: $n=41$

miR-135a: $n=42$

During both periods, i.e. with or without theobromine, miR-92a levels were significantly higher after meal consumption [(+1.21;95\% CI: 0.74, 1.67; p<0.001); (+0.43; 95\% CI: 0.16, 0.69; p<0.01), respectively]. MiR-223 levels were also increased after the meal intake [(+1.79; 95\% CI: 0.89, 2.68; p<0.001); (+2.33; 95\% CI: 1.32, 3.35; 
$\mathrm{p}<0.001)$, respectively]. Finally, miR-135a levels were increased during the theobromine period after test meal consumption $(+1.08 ; 95 \%$ CI: $0.62,1.54 ; \mathrm{p}<0.001)$, while only a trend was observed during the control period ( +0.23 ; $95 \%$ CI: $-0.01,0.46$; $\mathrm{p}=0.06$ ). Postprandial changes were significantly higher after the theobromine supplementation for miR-92a and miR-135a [ $(+0.85$; 95\% CI: 0.34, 1.36; $\mathrm{p}<0.01)$; $(+0.78 ; 95 \%$ CI: $0.32,1.25 ; \mathrm{p}<0.01)$, respectively], as compared to the placebo period. No difference in the postprandial responses between the two periods was found for miR-223 (-0.37; 95\% CI: -1.71, 0.97; $\mathrm{p}=0.58)$ (Figure 2).

\section{Correlation between changes in miRNAs levels with changes in biochemical parameters and cholesterol efflux capacity}

As miR-92a levels significantly decreased after 4 weeks of theobromine consumption, correlations between fasting changes in miR-92a levels and fasting changes in biochemical parameters and cholesterol efflux were calculated. No statistically significant associations were found, which was expected, as the parameters were not changed after the theobromine consumption.

During the placebo period, postprandial changes in miR-223 levels significantly correlated with age $(\mathrm{r}=-0.43, \mathrm{p} \leq 0.01)$ and with postprandial changes in TAG $(\mathrm{r}=0.31$, $\mathrm{p}<0.05$ ). No associations between postprandial changes in miR-92a and miR-135a with age and any of the changes in the clinical characteristics were observed. 


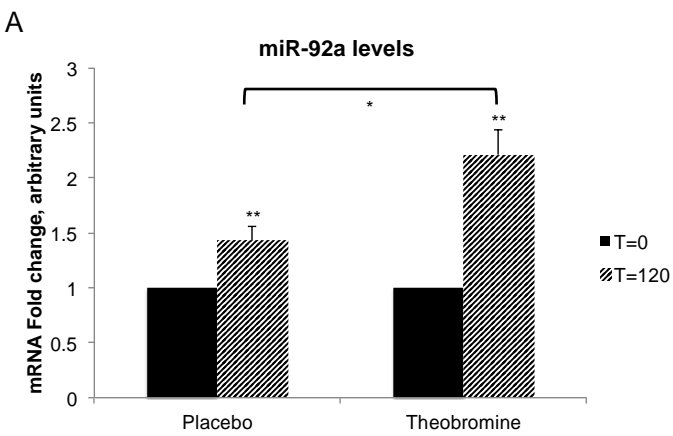

B

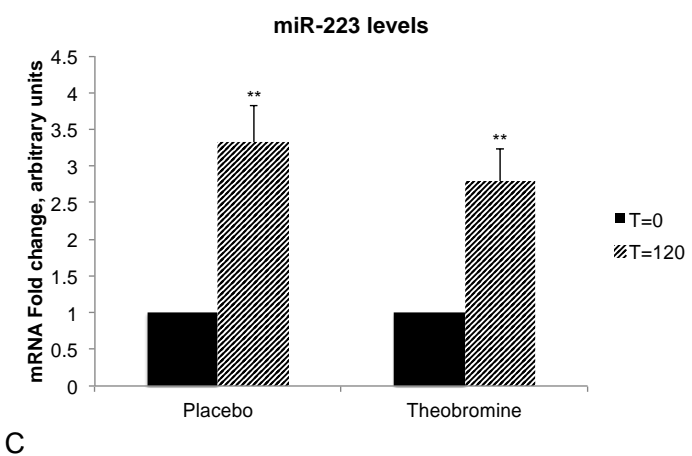

C

miR-135a levels

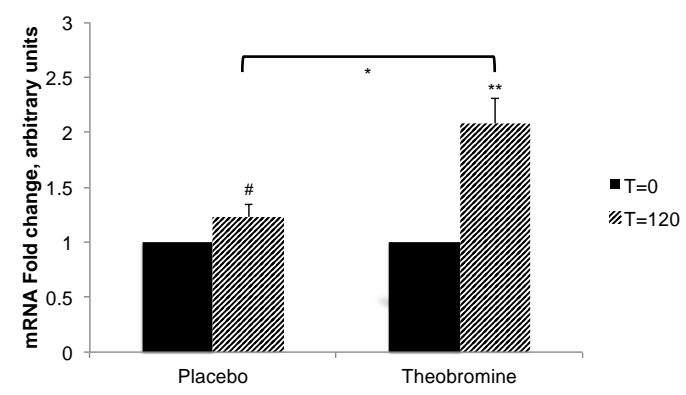

Figure 2: Fold changes of postprandial miRNAs levels 2 hours after high-meal consumption.

Postprandial gene expression ( $T=120)$ relative to the fasting gene expression $(T=0)$, in the placebo and the theobromine group for:

(A) miR-92a: Placebo: $n=41$; Theobromine: $n=43$

(B) miR-223: Placebo: $n=43$; Theobromine: $n=42$

(C) miR-135a: Placebo: $n=43$; Theobromine: $n=44$

Postprandial effect was assessed with one-samples t-test, with the test value set to 1: T=120 significantly different from $T=0 ;{ }^{*} p \leq 0.01 ;{ }^{* *} p \leq 0.001 ;{ }^{*}=0.06$

Difference in changes between theobromine and placebo was performed with a paired-samples $t$-test: significantly different from change in placebo after meal consumption 


\section{Discussion}

In this study, a daily intake of theobromine for 4 weeks did not affect fasting HDLmediated cholesterol efflux capacity. Also, fasting miR-223 levels, which have been related to cholesterol efflux in vitro [7], and miR-135a levels were unchanged. However, theobromine intake decreased fasting miR-92a levels. No correlation between changes in miR-92a and cholesterol efflux was found. In addition, the intake of the high fat meal increased postprandial cholesterol efflux capacity, as well as levels of the three selected miRNAs, i.e. miR-92a, miR-223 and miR-135a, but again no correlation between postprandial changes in efflux and miR expression was found.

Cocoa may protect against CVD by affecting HDL metabolism $[15,23]$. It is unlikely however that these potential effects are mediated by changing concentrations of HDL-cholesterol. First of all, effects of cocoa on HDL-cholesterol are not consistent [10-12], while it is debatable that increasing HDL-cholesterol protects against CVD [13]. We therefore postulated that cocoa may mediate HDL functionality, here defined as cholesterol efflux capacity. To the best of our knowledge, effects of cocoa and cocoa-rich products such as dark chocolate on cholesterol efflux have never been investigated. However, it has been suggested that flavonoids from cocoa, including flavones, isoflavones, flavanones, flavonols, flavanols, and anthocyanins [24], may improve cholesterol efflux capacity in vitro [25]. Extrapolation of these in vitro findings to the human in vivo situation is difficult, as the doses used in these in vitro studies were high as compared to the estimated dietary flavonoids intake from for example the US recommendations [26], while bioavailability and bioconversion of these compounds is not taken into account [25]. In two clinical studies, dietary anthocyanin increased cholesterol efflux capacity from macrophages in dyslipidemic [27] and hypercholesterolemic [28] adults. In contrast, consumption of isoflavones for 3 months did not affect SR-BI-[29] and ABCA1-mediated cholesterol efflux [30] in postmenopausal women. Thus, although some studies suggest that certain flavonoids have beneficial effects, our results do not suggest that theobromine consumption affects the ability of HDL to stimulate ABCA1-mediated cholesterol efflux from macrophages.

Although postprandial dietary effects on cholesterol efflux have not been largely explored, it has been suggested that cholesterol efflux increases during the postprandial phase [31]. More recently, it was shown that ABCA1- and SR-BImediated efflux pathways were increased at $4 \mathrm{~h}$ and $8 \mathrm{~h}$ after the meal, while ABCG1mediated efflux was unchanged [32]. Moreover, acute walnut oil consumption increased postprandial cholesterol efflux from THP-1 human monocytes 4 hours 
after the intake, as compared to control [33]. Comparable results have been reported for the acute consumption of whole walnuts using J774 macrophages [34]. The fatty acid composition of the meal may be important, as a test meal with a high content of monounsaturated fatty acids increased cholesterol efflux from $4 \mathrm{~h}$ to $8 \mathrm{~h}$ after the intake, while polyunsaturated fatty acid had no effect. Changes in efflux capacity were associated with changes in the phospholipid composition of HDL particles [35]. We also found a postprandial increase in cholesterol efflux $2 \mathrm{~h}$ after the intake of the test meal. However, this effect was not changed by theobromine consumption. As the phospholipid content of HDL is a significant predictor of cholesterol efflux capacity [36], the intake of a high-fat meal may lead to the production of more functional HDL particles, via an enrichement in in phospholipids. In addition, pre $\beta$ HDL concentrations have been shown to increase in the postprandial state in overweight and obese subjects [37]. As pre $\beta$-HDLs are the primary acceptor of cholesterol via the ABCA1 transporter, it is likely that pre $\beta$-HDLs are partly responsible for the postprandial increase in cholesterol efflux.

Effects of theobromine on miR-92a, miR-223 and miR-135a, three miRNAs known to be abundantly present in HDL particles, were investigated in the present study. MiR-92a expression in serum and plasma was increased in unstable CAD patients, suggesting that this miRNA could serve as biomarker for the detection of CAD [6, 38]. Further, in plasma, miR-135a levels were decreased in subjects with familial hypercholesterolemia, while miR-223 levels were increased [3]. MiR-223 plays a role in cholesterol homeostasis and increased ABCA1-mediated cholesterol efflux from hepatocytes [7]. However, our results do not support this finding in J774 macrophages. The associations between cholesterol efflux and miR-92a and miR135a have never been investigated, but we did not find any association between miR92a and miR-135a with cholesterol efflux capacity.

Exosomes also carry specific miRNAs [3]. As exosomes and HDL particles have overlapping densities, the isolation of pure HDL particles is challenging [39]. MiR223 [40] and miR-92a [41] are present in exosomes, which might have contributed to the present results. To our knowledge, the presence of miR-135a in human exosomes has not been examined.

Fasting miR-223 and miR-135a levels can be changed by dietary intervention. Four weeks of dietary trans fat consumption, from industrial or ruminant origin, increased concentrations of HDL-miR-223 and miR-135a. Variations in miR-223 levels were associated with a decrease in HDL-C and an increase in CRP concentrations, while variations in miR-135a levels were associated with an increase in LDL-C and TAG concentrations [40]. In addition, HDL-miR-223 levels were reduced after 12 weeks weight loss using a high-protein diet, independent of HDL 
composition and size [41]. In our present study, we found no effect of theobromine on miR-135a and miR-223, but fasting miR-92a levels were decreased. Although it is tempting to speculate about positive effects related to this effect since miR-92a might be involved in angiogenesis and atherosclerosis [6, 38, 42, 43], longer-term intervention studies specifically targeting miR-92a are needed before it can be concluded whether these effects of theobromine are beneficial or not. However, when found protective, changes in miR-92a levels are not associated with HDLefflux capacity, apoA-I and TAG concentrations, as they did not change after the 4 weeks theobromine consumption. A slight increase in HDL-C concentrations was found after the theobromine supplementation, but this was also not associated with changes in miR-92a. Changes in HDL particles size and/or composition could be a possible explanation. However, we have recently reported that theobromine consumption for 4 weeks was not associated with changes in the cholesterol concentrations in total HDL particles, as well as in any of the HDL subfractions [44].

This is the first time that the postprandial responses of miR-92a, miR-223 and miR135a have been investigated. Following meal intake, both miR-92a and miR-223 levels were significantly increased, while those of miR-135a tended to increase. Postprandial changes in miR-92a and miR-135a were significantly higher after the 4 weeks theobromine consumption. These findings are difficult to explain and were not related to postprandial changes in concentrations of HDL-C, apoA-I and TAG, or cholesterol efflux, which did not change. Not much is known regarding changes of miRNAs levels during the postprandial phase. A study performed in rats investigated the effect of proanthocyanidin consumption on miRNAs levels, and found a decreased in miR-122 and miR-33a levels in the liver $1 \mathrm{~h}$ after intake [45]. In rainbow trout, levels of miRNAs involved in insulin signaling were increased $4 \mathrm{~h}$ after meal intake [46]. However, no clear explanations for these postprandial changes were given but it was suggested that it might be attributed to changes in nutrients composition or to variation in hormonal factors. Indeed, the postprandial increase in miRNAs levels might be related to the normal increase in insulin observed after meal intake, as our selected miRNAs have been shown to be involved in the regulation of insulin biosynthesis and signaling pathway [47, 48]. In addition, miRNAs are found in food products, and can be absorbed [49-52]. However, if the specific miRNAs studied in the present study are associated with food products and theobromine is not known. In addition, if the postprandial increases in miRNAs levels are due to absorption from dietary sources or to an increase in the expression of genes coding for miRNAs needs further investigations. In this study, a high fat meal was used for the postprandial test. It would be of interest to investigate if the same postprandial effects on miRNAs levels depend on macronutrient composition. 
In conclusion, the present study shows that high-fat meal intake increases postprandial cholesterol efflux capacity, as well as miRNAs levels. However, 4 weeks of theobromine consumption did not change HDL-mediated cholesterol efflux capacity at baseline and postprandially. In addition, theobromine could exert anti-atherogenic properties by reducing miR-92a levels. We cannot exclude that miRNAs carried by exosomes might have contributed to the present results. 


\section{Supplementary data}

Table 1: Composition of the test drinks $(20 \mathrm{ml})$

\begin{tabular}{lcc}
\hline & Placebo & Theobromine \\
\hline Theobromine (mg) & - & 500 \\
Microcrystalline cellulose (mg) & 500 & - \\
Methyl cellulose (mg) & 150 & 150 \\
Sucralose (mg) & 10 & 10 \\
Sodium benzoate (mg) & 100 & 100 \\
Anise 0.1\% (mg) & 20 & 20 \\
Water & Until 20 g & Until 20g \\
\hline
\end{tabular}




\section{References}

1. Lai, E.C., Micro RNAs are complementary to 3' UTR sequence motifs that mediate negative post-transcriptional regulation. Nat Genet, 2002. 30(4): p. 363-4.

2. Creemers, E.E., A.J. Tijsen, and Y.M. Pinto, Circulating microRNAs: novel biomarkers and extracellular communicators in cardiovascular disease? Circ Res, 2012. 110(3): p. 48395.

3. Vickers, K.C., et al., MicroRNAs are transported in plasma and delivered to recipient cells by high-density lipoproteins. Nat Cell Biol, 2011. 13(4): p. 423-33.

4. Tabet, F., et al., HDL-transferred microRNA-223 regulates ICAM-1 expression in endothelial cells. Nat Commun, 2014. 5: p. 3292.

5. Wagner, J., et al., Characterization of levels and cellular transfer of circulating lipoproteinbound microRNAs. Arterioscler Thromb Vasc Biol, 2013. 33(6): p. 1392-400.

6. Niculescu, L.S., et al., MiR-486 and miR-92a Identified in Circulating HDL Discriminate between Stable and Vulnerable Coronary Artery Disease Patients. PLoS One, 2015. 10(10): p. e0140958.

7. Vickers, K.C., et al., MicroRNA-223 coordinates cholesterol homeostasis. Proc Natl Acad Sci U S A, 2014. 111(40): p. 14518-23.

8. Buitrago-Lopez, A., et al., Chocolate consumption and cardiometabolic disorders: systematic review and meta-analysis. Bmj, 2011. 343: p. d4488.

9. Hooper, L., et al., Effects of chocolate, cocoa, and flavan-3-ols on cardiovascular health: a systematic review and meta-analysis of randomized trials. Am J Clin Nutr, 2012. 95(3): p. 740-51.

10. Shrime, M.G., et al., Flavonoid-rich cocoa consumption affects multiple cardiovascular risk factors in a meta-analysis of short-term studies. J Nutr, 2011. 141(11): p. 1982-8.

11. Jia, L., et al., Short-term effect of cocoa product consumption on lipid profile: a meta-analysis of randomized controlled trials. Am J Clin Nutr, 2010. 92(1): p. 218-25.

12. Tokede, O.A., J.M. Gaziano, and L. Djousse, Effects of cocoa products/dark chocolate on serum lipids: a meta-analysis. Eur J Clin Nutr, 2011. 65(8): p. 879-86.

13. Rader, D.J. and G.K. Hovingh, HDL and cardiovascular disease. Lancet, 2014. 384(9943): p. 618-25.

14. Kelly, C.J., Effects of theobromine should be considered in future studies. Am J Clin Nutr, 2005. 82(2): p. 486-7; author reply 487-8.

15. Neufingerl, N., et al., Effect of cocoa and theobromine consumption on serum HDLcholesterol concentrations: a randomized controlled trial. Am J Clin Nutr, 2013. 97(6): p. 1201-9.

16. Karpe, F., Postprandial lipoprotein metabolism and atherosclerosis. J Intern Med, 1999. 246(4): p. 341-55.

17. Cohn, J.S., Are we ready for a prospective study to investigate the role of chylomicrons in cardiovascular disease? Atheroscler Suppl, 2008. 9(2): p. 15-8.

18. Smolders, L., et al., Theobromine does not affect postprandial lipid metabolism and duodenal gene expression, but has unfavorable effects on postprandial glucose and insulin responses in humans. Clin Nutr, 2017. 
19. Sankaranarayanan, S., et al., A sensitive assay for ABCA1-mediated cholesterol efflux using BODIPY-cholesterol. J Lipid Res, 2011. 52(12): p. 2332-40.

20. Davidson, W.S., et al., The effects of apolipoprotein B depletion on HDL subspecies composition and function. J Lipid Res, 2016. 57(4): p. 674-86.

21. Mitchell, P.S., et al., Circulating microRNAs as stable blood-based markers for cancer detection. Proc Natl Acad Sci U S A, 2008. 105(30): p. 10513-8.

22. Livak, K.J. and T.D. Schmittgen, Analysis of relative gene expression data using real-time quantitative PCR and the 2(-Delta Delta C(T)) Method. Methods, 2001. 25(4): p. 402-8.

23. Khan, N., et al., Regular consumption of cocoa powder with milk increases HDL cholesterol and reduces oxidized LDL levels in subjects at high-risk of cardiovascular disease. Nutr Metab Cardiovasc Dis, 2012. 22(12): p. 1046-53.

24. Oracz, J., D. Zyzelewicz, and E. Nebesny, The content of polyphenolic compounds in cocoa beans (Theobroma cacao L.), depending on variety, growing region, and processing operations: a review. Crit Rev Food Sci Nutr, 2015. 55(9): p. 1176-92.

25. Millar, C.L., Q. Duclos, and C.N. Blesso, Effects of Dietary Flavonoids on Reverse Cholesterol Transport, HDL Metabolism, and HDL Function. 2017. 8(2): p. 226-239.

26. Chun, O.K., S.J. Chung, and W.O. Song, Estimated dietary flavonoid intake and major food sources of U.S. adults. J Nutr, 2007. 137(5): p. 1244-52.

27. Qin, Y., et al., Anthocyanin supplementation improves serum LDL- and HDL-cholesterol concentrations associated with the inhibition of cholesteryl ester transfer protein in dyslipidemic subjects. Am J Clin Nutr, 2009. 90(3): p. 485-92.

28. Zhu, Y., et al., Anthocyanin supplementation improves HDL-associated paraoxonase 1 activity and enhances cholesterol efflux capacity in subjects with hypercholesterolemia. J Clin Endocrinol Metab, 2014. 99(2): p. 561-9.

29. Tormala, R.M., et al., Serum cholesterol efflux potential in postmenopausal women treated with isolated isoflavones. Menopause, 2006. 13(1): p. 96-101.

30. Badeau, R., et al., Effect of isolated isoflavone supplementation on ABCA1-dependent cholesterol efflux potential in postmenopausal women. Menopause, 2007. 14(2): p. 293-9.

31. Syeda, F., et al., Postprandial variations in the cholesteryl ester transfer protein activity, phospholipid transfer protein activity and plasma cholesterol efflux capacity in normolipidemic men. Nutr Metab Cardiovasc Dis, 2003. 13(1): p. 28-36.

32. Bellanger, N., et al., Functionality of postprandial larger HDL2 particles is enhanced following CETP inhibition therapy. Atherosclerosis, 2012. 221(1): p. 160-8.

33. Zhang, J., et al., Walnut oil increases cholesterol efflux through inhibition of stearoyl CoA desaturase 1 in THP-1 macrophage-derived foam cells. Nutr Metab (Lond), 2011. 8: p. 61.

34. Berryman, C.E., et al., Acute consumption of walnuts and walnut components differentially affect postprandial lipemia, endothelial function, oxidative stress, and cholesterol efflux in humans with mild hypercholesterolemia. J Nutr, 2013. 143(6): p. 788-94.

35. Sakr, S.W., et al., Oleic acid-rich fats increase the capacity of postprandial serum to promote cholesterol efflux from Fu5AH cells. Biochim Biophys Acta, 1996. 1300(1): p. 49-55.

36. Agarwala, A.P., et al., High-Density Lipoprotein (HDL) phospholipid content and cholesterol efflux capacity are reduced in patients with very high HDL cholesterol and coronary disease. Arterioscler Thromb Vasc Biol, 2015. 35(6): p. 1515-9. 
37. Attia, N., et al., Impact of android overweight or obesity and insulin resistance on basal and postprandial SR-BI and ABCA1-mediated serum cholesterol efflux capacities. Atherosclerosis, 2010. 209(2): p. 422-9.

38. Ren, J., et al., Signature of circulating microRNAs as potential biomarkers in vulnerable coronary artery disease. PLoS One, 2013. 8(12): p. e80738.

39. Yuana, Y., et al., Co-isolation of extracellular vesicles and high-density lipoproteins using density gradient ultracentrifugation. J Extracell Vesicles, 2014. 3.

40. Desgagne, V., et al., Variations in HDL-carried miR-223 and miR-135a concentrations after consumption of dietary trans fat are associated with changes in blood lipid and inflammatory markers in healthy men - an exploratory study. Epigenetics, 2016. 11(6): p. 438-48.

41. Tabet, F., et al., High-Density Lipoprotein-Associated miR-223 Is Altered after DietInduced Weight Loss in Overweight and Obese Males. PLoS One, 2016. 11(3): p. e0151061.

42. Bonauer, A., et al., MicroRNA-92a controls angiogenesis and functional recovery of ischemic tissues in mice. Science, 2009. 324(5935): p. 1710-3.

43. Loyer, X., et al., Inhibition of microRNA-92a prevents endothelial dysfunction and atherosclerosis in mice. Circ Res, 2014. 114(3): p. 434-43.

44. Jacobs, D.M., et al., Effect of Theobromine Consumption on Serum Lipoprotein Profiles in Apparently Healthy Humans with Low HDL-Cholesterol Concentrations. Front Mol Biosci, 2017. 4: p. 59.

45. Baselga-Escudero, L., et al., Grape seed proanthocyanidins repress the hepatic lipid regulators miR-33 and miR-122 in rats. Mol Nutr Food Res, 2012. 56(11): p. 1636-46.

46. Mennigen, J.A., et al., Postprandial regulation of hepatic microRNAs predicted to target the insulin pathway in rainbow trout. PLoS One, 2012. 7(6): p. e38604.

47. Setyowati Karolina, D., et al., miR-25 and miR-92a regulate insulin I biosynthesis in rats. RNA Biol, 2013. 10(8): p. 1365-78.

48. Chakraborty, C., et al., Influence of miRNA in insulin signaling pathway and insulin resistance: micro-molecules with a major role in type-2 diabetes. Wiley Interdiscip Rev RNA, 2014. 5(5): p. 697-712.

49. Baier, S.R., et al., MicroRNAs are absorbed in biologically meaningful amounts from nutritionally relevant doses of cow milk and affect gene expression in peripheral blood mononuclear cells, HEK-293 kidney cell cultures, and mouse livers. J Nutr, 2014. 144(10): p. 1495-500.

50. Wagner, A.E., et al., Food derived microRNAs. Food Funct, 2015. 6(3): p. 714-8.

51. Zempleni, J., et al., Gene regulation by dietary microRNAs. Can J Physiol Pharmacol, 2015. 93(12): p. 1097-102.

52. Cui, J., et al., Nutrition, microRNAs, and Human Health. Adv Nutr, 2017. 8(1): p. 105112.

53. Tomasetti, M., et al., Clinical significance of circulating miR-126 quantification in malignant mesothelioma patients. Clin Biochem, 2012. 45(7-8): p. 575-81. 


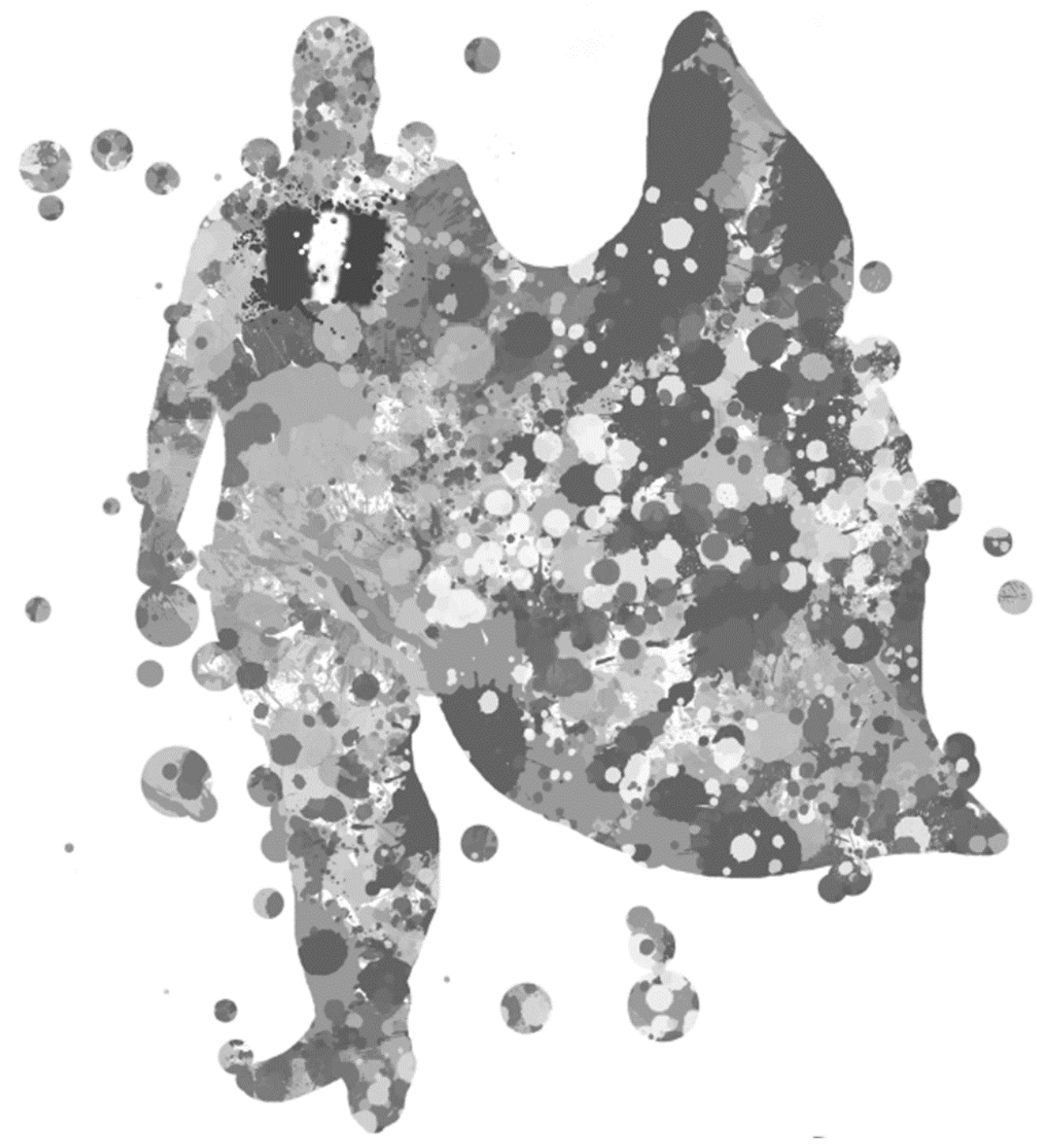




\section{Chapter 5}

\section{Effects of plant sterol and stanol}

ester enriched margarine consumption on plasma

\section{cholesterol efflux capacity, CETP}

\section{mass and $C E$ transfer}

Charlotte P.J. Talbot, Ronald P. Mensink, Dieter Lutjohann, Andreas Ritsch, Jogchum Plat

To be submitted 


\begin{abstract}
Background and Aim: Plant sterols and stanols consumption lower low-density lipoproteins cholesterol (LDL-C) concentrations, but may also affect high-density lipoprotein cholesterol (HDL-C) concentrations. However, effects on parameters related to HDL metabolism and functionality have hardly been studied. The aim of this study was therefore to investigate effects of plant sterol and stanol intake on HDL-related parameters, such as cholesterol efflux capacity, and cholesteryl ester transfer protein (CETP) mass and activity.
\end{abstract}

Methods: Data from 2 earlier studies with healthy subjects (BMI $\leq 32 \mathrm{~kg} / \mathrm{m} 2$; aged between 18-65 years) were used. In study 1, 24 men and 42 women consumed for 8 weeks $3.9 \mathrm{~g} /$ day plant stanols. In study 2, 26 men and 15 women consumed for 85 weeks $2.5 \mathrm{~g} /$ day plant stanols or sterols. Cholesterol efflux was measured in ${ }^{3} \mathrm{H}$ labeled murine J774 macrophages. CETP mass was measured by ELISA and CETP activity by quantifying the transfer of cholesteryl esters from radiolabeled exogenous HDL to apoB-containing lipoproteins.

Results: Serum plant sterol concentrations were positively associated with serum HDL-C concentrations. Plant stanol ester consumption significantly decreased CETP mass by $0.15 \mu \mathrm{g} / \mathrm{mL}$ in both studies, but did not affect cholesterol efflux and CETP activity. No effect of the plant sterol esters was found on any of the HDL-related parameters examined.

Conclusion: Plant stanol intake significantly decreased CETP mass, but not CETP activity and cholesterol efflux, while no effects of plant sterols were found. Further studies in populations with low HDL-C are needed to better understand their effects on HDL metabolism and functionality. 


\section{Introduction}

Different foods like margarines and yogurt drinks enriched with plant sterol or stanol esters are well recognized for their serum total and low-density lipoproteins cholesterol (LDL-C) lowering effects. Therefore, these foods are part of various dietary guidelines to lower cardiovascular diseases (CVD) risk [1]. Although the earlier studies did not indicate that serum triacylglycerol (TAG) concentrations were affected by plant sterol or stanol esters, later studies showed that effects did depend on baseline TAG concentrations. No effects were evident in normotriglyceridemic subjects, but in subjects with elevated serum TAG concentrations, consumption of both plant sterols and stanols lowered TAG concentrations, in addition to their LDL$C$ lowering effect [1-4]. The exact mechanism underlying this TAG-lowering effect has not yet been established, but might relate to changes in hepatic very low-density lipoproteins (VLDL) production [5, 6] and/or intestinal fat absorption [7]. Whether plant sterols and stanols also have an effect on high-density lipoproteins (HDL) metabolism is less clear, as no changes in serum HDL cholesterol (HDL-C) and/or apoA-I concentrations were observed in individual studies so far. However, as TAG and HDL metabolism are closely related, effects on HDL cannot be excluded and may be found when studies in responsive groups are performed. After combining the individual data from 5 intervention studies, we have previously suggested that HDL-C concentrations increased in subjects with low HDL-C concentrations at baseline [2]. Whether this finding is real needs to be confirmed or refuted in intervention studies specifically designed for this purpose and using the optimal target population. However, given the current discussion whether changing HDL metabolism is relevant in the context of managing CVD risk, an additional question that needs to be answered is whether plant sterols and stanols do have an effect on more functional HDL-related parameters. Since HDL functionality can also be modulated even without changing serum HDL-C concentrations, we here evaluated the effects of plant sterol and stanol ester consumption on HDL-related parameters such as cholesterol efflux capacity, CETP mass and CE transfer activity.

\section{Materials and Methods}

\section{Subjects}

The samples used in this study were from two studies previously carried out at Maastricht University. In the first study, the effects of vegetable oil based versus wood based stanol ester mixtures on serum lipids and hemostatic factors in nonhypercholesterolemic subjects were studied. One hundred and twelve healthy subjects were included in the original study. However, stored samples were only 
available for 24 men and 42 women. To be able to participate in this first study, subjects had to be healthy with a BMI $<30 \mathrm{~kg} / \mathrm{m}^{2}$ and aged between $18-65$ years. Full details of the inclusion criteria have been published before [8]. In the second study, the effects of long-term plant sterol or stanol ester consumption on lipid and lipoprotein metabolism were examined in subjects on medically prescribed stable statin treatment, already before inclusion. Samples of 26 men and 15 women were still available, out of the original 54 subjects that completed that study. To be eligible to participate in the second study, all subjects had to be healthy with a BMI $\leq 32$ $\mathrm{kg} / \mathrm{m}^{2}$ and aged between 18-65 years. Full details of the inclusion criteria have been published before [9]. Both studies were conducted according to the ethical guidelines of the 1975 Declaration of Helsinki and approved by the Ethics Committee of Maastricht University Medical Centre. Written informed consent was obtained from all participants before entering the study.

\section{Studies design}

In the first study, subjects had to replace their own margarine during the 4 weeks run-in period by a low erucic acid rapeseed oil based margarine ( $74 \%$ absorbable fat) and had to consume daily minimally $20 \mathrm{~g}$ of margarine. At the end of the 4 weeks run-in period, subjects were randomly allocated to one of the three experimental groups. For the following 8 weeks, the first group continued to consume the control margarine $(n=26)$, the second group $(n=15)$ consumed the same rapeseed-oil based margarine enriched with a vegetable oil based plant stanol ester mixture, and the third group $(n=25)$ the same rapeseed-oil based margarine enriched with a wood based stanol ester mixture. In the second and the third group, subjects consumed an average of $3.9 \mathrm{~g}$ of plant stanols per day [8].

In the second study, the participants had to replace their own margarine during the 5 weeks run-in period with the experimental 'light' margarine ( $40 \%$ absorbable fat), and had to consume daily minimally $30 \mathrm{~g}$ of margarine. At the end of the run-in period, subjects were randomly allocated to one of the three experimental groups. For the following 85 weeks, the first group continued to consume the control margarine $(n=15)$, the second group $(n=14)$ consumed a plant-sterol enriched margarine and the third group $(n=12)$ a plant-stanol enriched margarine. For the second and third group, the daily intake of $30 \mathrm{~g}$ of margarine resulted in an intake of $2.5 \mathrm{~g}$ plant sterols or stanols per day [9].

Plant sterols and plant stanols were obtained by transesterification of free plants sterols and stanols with sunflower oil based fatty acids for the plant sterols or with rapeseed oil based fatty acids for the plant stanols. Plant sterols contained $49 \%$ sitosterol, 31\% campesterol and 16\% stigmasterol. Plant stanols consisted of 70\% 
sitostanol and 30\% campestanol for the vegetable-oil based stanol and of $93 \%$ sitostanol and $7 \%$ campestanol for the wood-based stanol. The detailed composition of the experimental margarines has been previously described $[8,9]$.

\section{Blood sampling}

For the first study, fasting blood samples were collected by venipuncture after 4 weeks (end of the run-in period) and 12 weeks (end of the experimental period). Serum was obtained by centrifugation at $2000 \times \mathrm{g}$ for 30 minutes at $4^{\circ} \mathrm{C}$ [8]. In the second study, fasting blood samples collected by venipuncture were obtained after 5 weeks (end of the run-in-period) and 90 weeks (end of the experimental period). Blood was collected in a $4 \mathrm{~mL}$ EDTA tube and then centrifuged at $2000 \times \mathrm{g}$ for 30 minutes at $4^{\circ} \mathrm{C}$ to obtain plasma [9].

\section{Biochemical measurements}

Measuring serum HDL-C concentrations was done by the precipitation of apoBcontaining lipoproteins after adding a mixture of phosphotungstic acid and magnesium ions to the sample (CHOD/PAP method; Roche Diagnostics System, Hofmann-La Roche Ltd., Basel, Swiss). Measurement of serum apoA-I concentrations was performed by using an immunoturbidimetric reaction (UNI-KIT ApoA-I, Roche, Basel). Analysis of serum TAG concentrations was done after correction for free glycerol concentrations (GPO-Trinder; Sigma Diagnostics, St Louis, USA). All samples from one subject were analyzed within the same run. Plant sterols (sitosterol and campesterol) and plant stanols (sitostanol and campestanol) were analyzed by gas chromatography in the first study and by gas-liquid chromatography-mass spectrometry (GC-MS) in the second study, as previously described [10]. Serum plant sterol and plant stanol concentrations were expressed as $\mathrm{x} 10^{2} \mu \mathrm{mol}$ per mmol total cholesterol.

To measure the ability of HDL particles to mediate cholesterol efflux from macrophages, radiolabeled- ${ }^{3} \mathrm{H}$-cholesterol loaded murine J774 macrophages were incubated with apolipoprotein B-depleted serum, as previously described [11]. The amount of radioactive cholesterol secreted from the loaded cells was quantified by liquid scintillation counting. Cholesterol efflux capacity was expressed as $\%$ of the efflux of a plasma pool. Known proteins involved in cholesterol efflux from macrophages, such as ABCA1, ABCG1 and SRB1 are an intrinsic part of the assay and therefore similar in all analyses. However, the ABCA1 transporter was upregulated with cyclic adenosine monophosphate (cAMP) to measure primarily 
ABCA1-mediated cholesterol efflux. However, the HDL fractions before and after plant sterol or stanol intervention that are added to these cells, are subject-specific.

CETP mass was quantified in plasma samples using an enzyme linked immunosorbent assay (ELISA) as previously described [12]. Briefly, a CETP-specific recombinant single-chain antibody was used as coating antibody and an affinitypurified polyclonal anti-CETP antibody as detection antibody. CE transfer measurements were performed, also as previously described. In short, by incubating whole plasma with radiolabeled HDL in order to measure the ability of exogenous HDL to transfer cholesteryl ester (CE) to apoB-containing lipoproteins [13, 14]. CE transfer was measured as the fraction of the total radiolabeled-CE transferred to apoB-containing lipoproteins compared with controls. Results are expressed as the percentage decrease of ${ }^{3} \mathrm{H}-\mathrm{CE}$ in the supernatant of total radiolabeled-CE.

\section{Statistics}

All analyses were adjusted for the factor study to adjust for differences in study characteristics. To examine cross-sectional associations, data from both studies in samples obtained at the end of the run-in periods were combined ( $n=107)$. Multiple linear regression was used to study associations among HDL-related parameters (HDL-C, apoA-I, TAG, cholesterol efflux capacity, CETP mass and CE transfer activity). Another multiple linear regression model was used to study associations between absolute serum plant sterol and stanol concentrations (sitosterol, campesterol, sitostanol and campestanol) with HDL-related parameters (cholesterol efflux capacity, CETP mass and CE transfer activity). As plant sterols and stanols are incorporated into lipoproteins, the latter analyses were adjusted for HDL-C and LDL-C concentrations. Differences between the control group and the intervention groups at the run-in period were determined using the independent t-test. Responses to the consumption of plant sterols or stanols for each subject were calculated as the difference between values obtained at the end of the experimental period and the end of the run-in period. Effects of the interventions were tested using the general linear model with adjustment for run-in values. Correlations between changes were calculated using multiple linear regression after adjustment for changes in HDL-C and LDL-C concentrations. A P value $\leq 0.05$ was considered significant. All statistical analyses were performed with SPSS 20.0 for Mac OS. 


\section{Results}

\section{Baseline characteristics and compliance}

Baseline characteristics of the study population are presented in the Table 1. Subjects in study 2 were on average older and had a higher BMI $(\mathrm{p} \leq 0.001)$ than subjects in study 1 . In the first study, changes in cholesterol-standardized levels of sitosterol and campesterol, and of sitostanol and campestanol were not different between the vegetable-oil based plant stanol ester group and the wood-based stanol ester group after the 8 weeks intervention period. Also, lipid and lipoprotein responses were comparable (Supplemental Table I). Therefore, these two intervention groups were combined $(\mathrm{n}=40)$.

As expected, the intake of plant stanols increased cholesterol-standardized levels of sitostanol $(\mathrm{p}<0.001)$ and campestanol $(\mathrm{p}<0.001)$ as compared with the control groups, and decreased those of sitosterol and campesterol in study 1 ( $\mathrm{p}<0.001$; Table 2). However, in study 2, stanol intake only decreased cholesterol-standardized levels of sitosterol $(\mathrm{p}<0.05)$, without affecting cholesterol-standardized levels of campesterol $(\mathrm{p}=0.372)$. In the second study, the intake of plant sterols increased cholesterolstandardized levels of sitosterol $(\mathrm{p}<0.001)$ and campesterol $(\mathrm{p}<0.001)$, as compared with the control group. Moreover, in the second study, changes in cholesterolstandardized levels of sitosterol and campesterol were significantly different between the sterol and the stanol groups $(\mathrm{p} \leq 0.001)$.

\section{Baseline correlations between HDL-related parameters}

After adjusting for the factor study, HDL-C concentrations were associated with apoA-I concentrations $(\beta=1.10 \mathrm{mmol} / \mathrm{L}$ per $\mathrm{g} / \mathrm{L} ; \mathrm{p}<0.001)$ and TAG concentrations $(\beta=-0.24 \mathrm{mmol} / \mathrm{L}$ per $\mathrm{mmol} / \mathrm{L} ; \mathrm{p}<0.001)$. ApoA-I tended to be negatively associated with TAG concentrations $(\beta=-0.07 \mathrm{~g} / \mathrm{L}$ per $\mathrm{mmol} / \mathrm{L} ; \mathrm{p}=0.06)$. Cholesterol efflux was associated with HDL-C $(\beta=22.2 \%$ per $\mathrm{mmol} / \mathrm{L} ; \mathrm{p} \leq 0.001)$ and apoA-I concentrations $(\beta=30.2 \%$ per $\mathrm{g} / \mathrm{L} ; \mathrm{p}<0.01)$. CE transfer was related to HDL-C concentrations $(\beta=-8.45$ $\%$ per $\mathrm{mmol} / \mathrm{L} ; \mathrm{p}<0.001)$, apoA-I concentrations $(\beta=-7.93 \%$ per $\mathrm{g} / \mathrm{L} ; \mathrm{p}<0.001)$, and TAG concentrations $(\beta=3.21 \%$ per mmo/L; $p<0.001)$. CETP mass was related to apoA-I concentrations $(\beta=0.34 \mu \mathrm{g} / \mathrm{mL}$ per $\mathrm{g} / \mathrm{L} ; \mathrm{p} \leq 0.05)$ and tended be negatively related to TAG concentrations $(\beta=-0.13 \mu \mathrm{g} / \mathrm{mL}$ per $\mathrm{mmol} / \mathrm{L}$; $\mathrm{p}<0.06)$. 


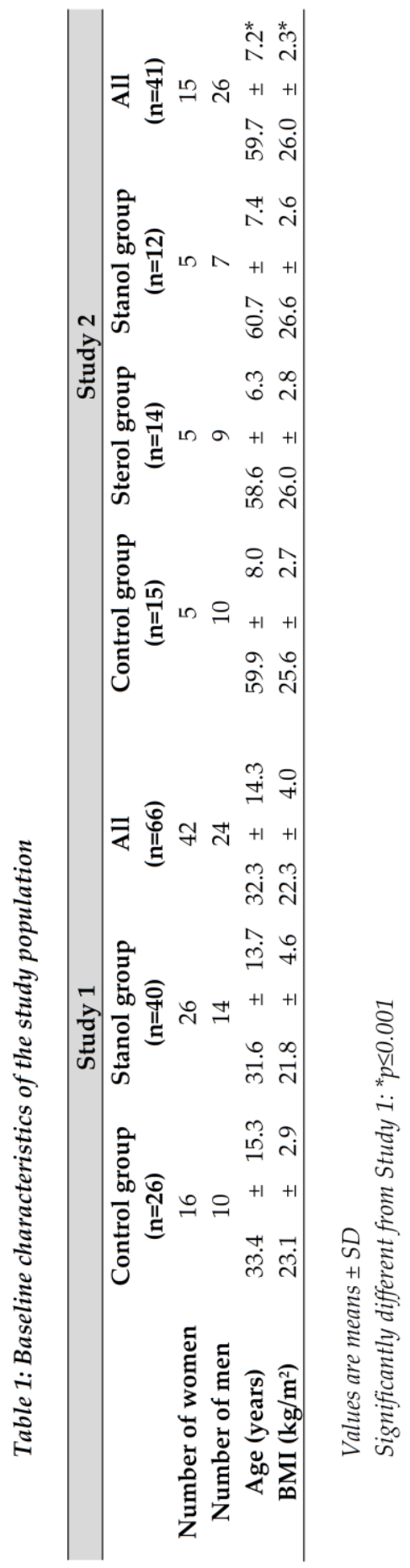




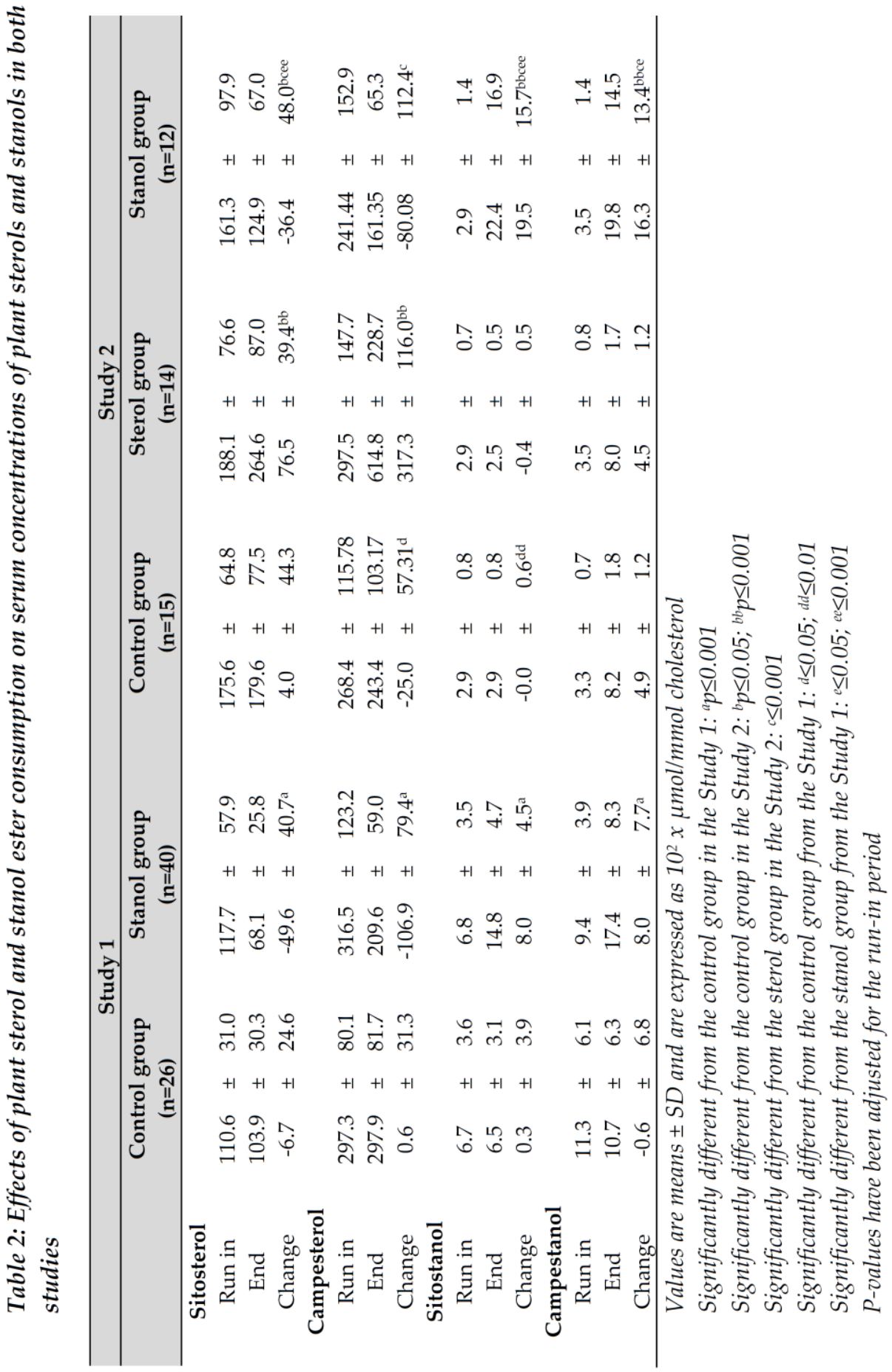




\section{Baseline associations between absolute serum plant sterol and stanol concentrations with HDL-related parameters}

At baseline and after adjusting for the factor study, absolute sitosterol concentrations were positively related with HDL-C concentrations $(\beta=2.64 \mu \mathrm{mol} / \mathrm{L}$ per $\mathrm{mmol} / \mathrm{L}$; $\mathrm{p}<0.01$ ), while no relation with LDL-C concentrations was found. Absolute campesterol concentrations tended to associate with HDL-C concentrations $(\beta=3.23$ $\mu \mathrm{mol} / \mathrm{L}$ per $\mathrm{mmol} / \mathrm{L} ; \mathrm{p}=0.08)$ and were significantly related with LDL-C concentrations $(\beta=1.99 \mu \mathrm{mol} / \mathrm{L}$ per $\mathrm{mmol} / \mathrm{L} ; \mathrm{p}<0.05)$. In addition, absolute sitostanol concentrations were significantly associated with both HDL-C $(\beta=-0.13 \mu \mathrm{mol} / \mathrm{L}$ per $\mathrm{mmol} / \mathrm{L} ; \mathrm{p}<0.01)$ and LDL-C $(\beta=0.06 \mu \mathrm{mol} / \mathrm{L}$ per $\mathrm{mmol} / \mathrm{L} ; \mathrm{p}<0.01)$ concentrations. No associations between absolute campestanol concentrations with HDL-C and LDL-C concentrations were found.

After adjusting for the factor study, and for HDL-C and LDL-C concentrations, multiple linear regression analysis showed that baseline CETP mass was significantly positively associated with baseline absolute campestanol concentration ns $(\beta=0.44 \mu \mathrm{g} / \mathrm{mL}$ per $\mu \mathrm{mol} / \mathrm{L} ; \mathrm{p}<0.05)$, while no associations between campestanol and cholesterol efflux or CE transfer activity were found. In addition, no associations between cholesterol efflux, CETP mass and CE transfer activity with baseline absolute concentrations of sitostanol, campesterol and sitosterol were found.

\section{Effects of plant sterol and plant stanol consumption on HDL-related parameters}

Changes in HDL-related parameters during consumption of plant sterol and stanol ester enriched diets are shown in Table 3. In both studies, values at the end of the run-in period were comparable between the control group and the intervention group. Plant stanol intake significantly reduced CETP mass by $-0.15 \pm 0.40 \mu \mathrm{g} / \mathrm{ml}$ in the first study $(\mathrm{p}<0.05)$ and by $-0.15 \pm 0.34 \mu \mathrm{g} / \mathrm{ml}$ in the second study $(\mathrm{p}<0.05)$ (Figure 1). However, CETP mass did not change after intake of plant sterols $(p=0.608)$. Further, changes between the sterol and the stanol groups were comparable $(\mathrm{p}=0.472)$. In both studies, CE transfer, cholesterol efflux, and HDL-C, apoA-I and TAG concentrations were not significantly changed after plant sterol or stanol intake. 


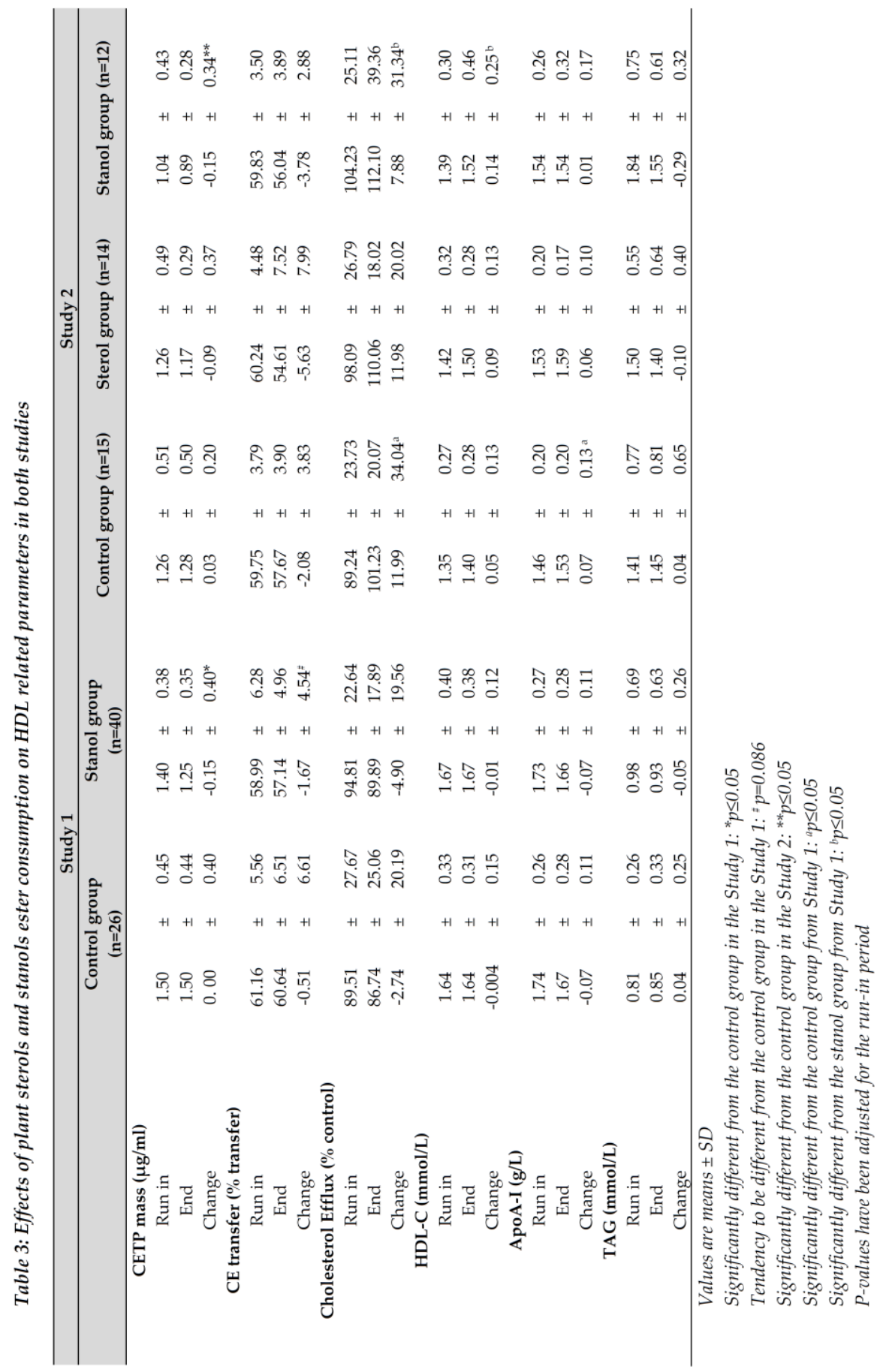




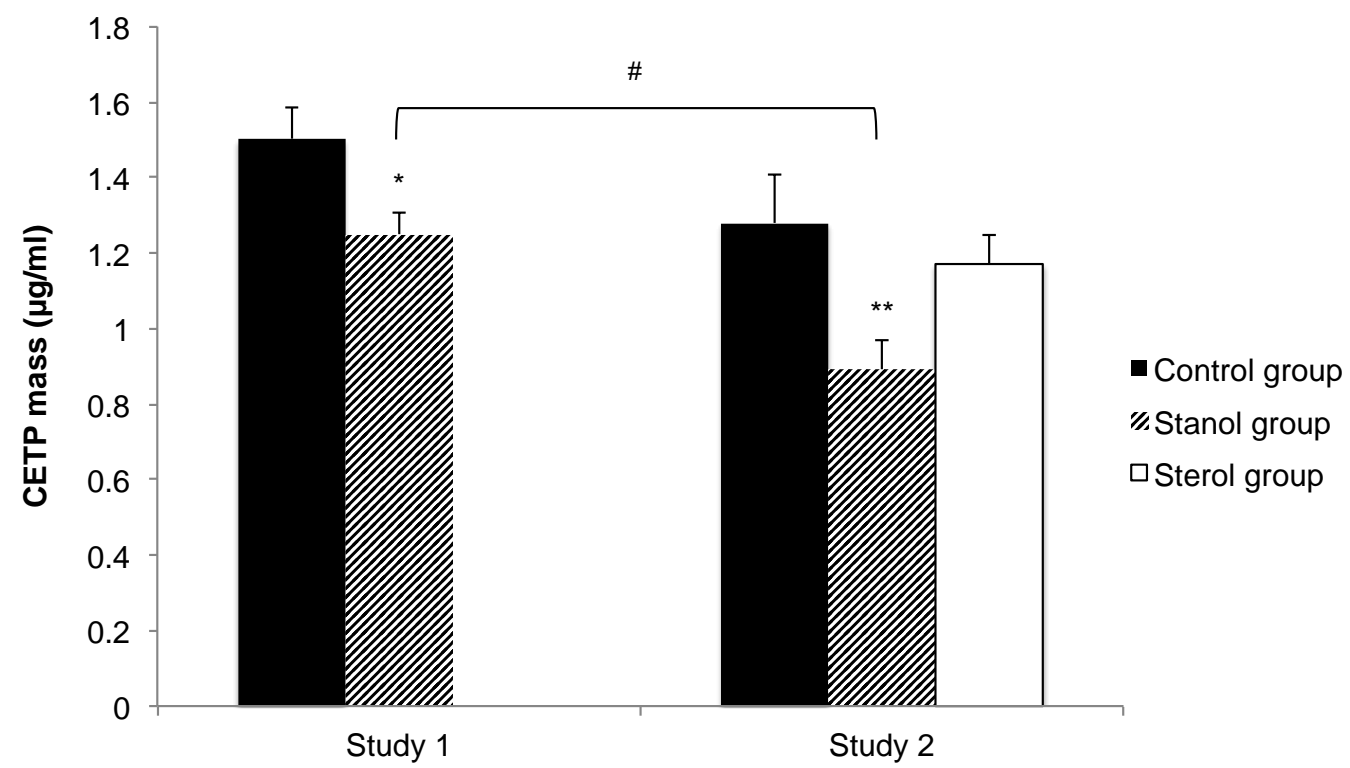

Figure 1: Effects of plant sterols and stanols consumption on CETP mass, in both studies.

Values are means $\pm S E$, at the end of the intervention (after 8 weeks for Study 1 and 85 weeks for Study 2).

Significantly different from the control group in the Study 1: ${ }^{*} p \leq 0.05$

Significantly different from the control group in the Study $2:{ }^{* *} p \leq 0.05$

Tendency to be different from the stanol group from Study 1: \#p=0.073

$P$-values have been adjusted for the run-in period

\section{Correlation between changes in campestanol concentrations and changes in CETP mass}

As absolute campestanol concentrations were significantly associated with CETP mass at baseline, and CETP mass decreased after plant stanol intake, correlation between changes in these two parameters was calculated. However, after adjusting for the factor study, and changes in HDL-C and LDL-C concentrations, no statistically significant association was found. 


\section{Discussion}

We here show that after plant sterol and stanol ester consumption, serum HDL-C and apoA-I concentrations, and cholesterol efflux capacity and CE transfer activity did not change. However, in the two independent intervention studies, plant stanol ester intake reduced CETP mass, while this was not found for plant sterol ester consumption. In addition, absolute serum campestanol concentrations were at baseline positively associated with CETP mass.

Effects of plant sterol and stanol ester consumption on HDL metabolism have not been evaluated into much detail so far. This is most likely related to the fact that in almost every individual study, no changes in serum HDL-C and/or apoA-I concentrations were observed. The fact that plant stanols do not lower serum HDL$\mathrm{C}$ concentrations is already intriguing, since lowering dietary cholesterol intake lowers serum HDL-C concentrations [15]. In addition, also treatment with neomycin and ezetimibe, which inhibit intestinal cholesterol absorption, lower serum HDL-C concentrations [16-18]. These interventions suggest that lowering cholesterol fluxes from the intestine to the circulation is linked with serum HDL-C concentrations. In other words, there is a clear rationale to hypothesize that plant sterol and stanol ester consumption affects serum HDL-C concentrations. Indeed, we earlier observed that combining the data from 5 intervention studies showed that HDL-C concentrations increased during plant stanol ester consumption, but only in subjects with low HDL$C$ concentrations at baseline [2]. Why plant stanol esters may even elevate serum HDL-C concentrations, whereas the other interventions lowering intestinal-derived cholesterol fluxes lowered serum HDL-C concentrations remains unanswered. What is clearly lacking is a study specifically designed to examine the effects of plant stanol ester on HDL metabolism in including subjects with low baseline HDL-C concentrations. We here tried to understand into more detail whether we could find supportive evidence to explain effects of plant stanol intake on HDL-C concentrations. Interestingly, CETP mass decreased in both studies in the stanol intervention groups. This is consistent with the observation of Homma et al., who showed that plant stanol ester consumption ( $2 \mathrm{~g} /$ day) lowered CETP mass by $6.1 \%$, although a higher stanol intake ( $3 \mathrm{~g} /$ day) lowered CETP mass by only $3.3 \%$ [19]. However, a decrease in CETP mass is difficult to explain, also because a positive correlation between absolute serum campestanol concentrations and CETP mass was observed at baseline. Based on this finding, it would be expected that consuming stanol-enriched products, which increases serum stanol concentrations even further, would increase CETP mass. In contrast to plant the stanol intervention, the plant sterol intervention was not associated with CETP mass. There are at least 
two potential explanations for this difference between the effects of plant stanols and sterols, i.e. (1) a direct effect of stanols on CETP or (2) an indirect effect via the decrease in sterol concentrations due to stanol intake. As neither sitosterol nor campesterol were associated with CETP mass at baseline, it is most likely that the decrease in CETP mass is directly mediated by the elevated stanol concentrations during stanol intake. However, the underlining mechanisms remains unknown. CETP expression is regulated by the liver X receptor (LXR) [20], and CETP concentrations indeed increased upon LXR activation [21]. It can therefore be suggested that the present results might be partly due to a direct effect of stanols on LXR, but this needs more detailed evaluation since plant sterols are also activate LXR [22].

A decrease in CETP mass is an interesting finding, since it is expected to improve the lipoprotein profile. CETP mass was previously reported to be negatively associated with HDL-C concentrations in subjects with low HDL-C and high TAG concentrations [23]. However, and similar to the study by Homma et al. [19] in subjects with normal HDL-C concentrations, the change in CETP mass in the present study was not translated into significant changes in HDL-C and TAG concentrations. However, CE transfer activity did not change after the stanol intake, which was supported by the lack of changes in HDL-C and TAG concentrations following the intervention. A possible explanation for the lack of effect might be attributed to the study population, which had normal HDL-C and TAG concentrations. As already discussed, plant stanol ester consumption, may only increase HDL-C concentrations in subjects with low HDL-C baseline concentrations and decrease those of TAG in subjects with high TAG baseline concentrations [2].

It has been reported several times that serum plant sterol concentrations are linked with serum HDL-C concentrations. Indeed, and in line with our findings, others also found that both sitosterol and campesterol concentrations are positively correlated with serum HDL-C concentrations [24-26]. For example, Nunes et al. showed that serum plant sterol concentrations were lower in subjects with low HDL-C concentrations as compared to their high HDL-C controls [25]. Within the circulation, plant sterols are incorporated into lipoprotein particles as their carriers. Although the absolute amount of plant sterols in the apoB containing lipoproteins is roughly 3-fold higher than in the non-apoB fraction, still a substantial amount of plant sterols is present in the HDL fraction. Moreover, the plant sterol / cholesterol ratio is higher in HDL particles than in LDL and VLDL particles [27]. We observed that sitosterol concentrations were associated with HDL-C concentrations, while no association was found with LDL-C concentrations. This suggests that sitosterol is preferentially associated with HDL particles, while campesterol is associated with 
both HDL and LDL. This raises the question whether the plant sterol content of HDL particles influences its functionality, as it becomes more and more recognized that HDL-particle composition is important determinant of its functionality. A wellknown example is that enrichment of HDL particles with serum amyloid A (SAA) during acute phase reactions results in less functional HDL particles [28]. However, more subtle variations in HDL particle composition, such as differences in phospholipid composition, also affect HDL functionality [29]. Further, the CETP inhibitor Dalcetrapid not only significantly elevated serum HDL-C concentrations, but also serum plant sterol concentrations [30] and cholesterol efflux [31-33]. Though speculative, these latter findings may suggest that elevated plant sterol concentrations improve cholesterol efflux capacity. Although we did not measure plant sterols concentrations specifically in the HDL fractions, no effect of the plant sterol intervention - in which plasma plant sterol concentrations clearly increased was found on ABCA1-mediated cholesterol efflux from macrophages. In addition, also plant stanol ester consumption, which lowers serum plant sterol concentrations did not affect cholesterol efflux. The present study is the first in which the potential effects of plant sterol or stanol ester consumption on HDL-mediated cholesterol efflux was evaluated. More studies in other populations are therefore needed to confirm or reject our findings.

In conclusion, we did not find evidence that plant sterol and stanol consumption affect cholesterol efflux capacity and CE transfer activity. Plant stanol ester intake reduced CETP mass, which was not found for plant sterol consumption. Further studies in populations with low serum HDL-C and high TAG concentrations are essential to definitely address the potential beneficial effects of plant sterol and stanol consumption on HDL metabolism and functionality. 


\section{Supplementary data}

Table I: Effects of woodstanol and vegetable oil based stanol ester consumption on serum concentrations of plant sterols and stanols, and on serum concentrations of lipids and lipoproteins.

\begin{tabular}{|c|c|c|c|c|c|c|}
\hline \multicolumn{7}{|c|}{ Study 1} \\
\hline & \multicolumn{3}{|c|}{$\begin{array}{l}\text { Woodstanol group } \\
(\mathrm{n}=25)\end{array}$} & \multicolumn{3}{|c|}{$\begin{array}{c}\text { Vegetable oil stanol group } \\
(n=15)\end{array}$} \\
\hline \multicolumn{7}{|c|}{ 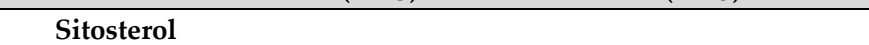 } \\
\hline Run in & 108.8 & \pm & 44.7 & 132.7 & \pm & 74.5 \\
\hline End & 68.8 & \pm & 26.3 & 66.9 & \pm & 25.9 \\
\hline Change & -40.0 & \pm & 25.3 & -65.7 & \pm & 55.4 \\
\hline \multicolumn{7}{|l|}{ Campesterol } \\
\hline Run in & 309.9 & \pm & 104.3 & 327.5 & \pm & 153.1 \\
\hline End & 214.8 & \pm & 60.1 & 200.9 & \pm & 58.1 \\
\hline Change & -95.1 & \pm & 57.8 & -126.6 & \pm & 105.8 \\
\hline \multicolumn{7}{|l|}{ Sitostanol } \\
\hline Run in & 7.5 & \pm & 4.0 & 5.7 & \pm & 2.2 \\
\hline End & 15.3 & \pm & 5.1 & 14.1 & \pm & 3.8 \\
\hline Change & 7.8 & \pm & 4.5 & 8.3 & \pm & 4.6 \\
\hline \multicolumn{7}{|l|}{ Campestanol } \\
\hline Run in & 9.0 & \pm & 3.7 & 9.9 & \pm & 4.4 \\
\hline End & 18.9 & \pm & 8.0 & 14.8 & \pm & 8.6 \\
\hline Change & 9.9 & \pm & 7.7 & 4.9 & \pm & 6.9 \\
\hline \multicolumn{7}{|l|}{ HDL-C (mmol/L) } \\
\hline Run in & 1.62 & \pm & 0.39 & 1.76 & \pm & 0.41 \\
\hline End & 1.62 & \pm & 0.35 & 1.76 & \pm & 0.41 \\
\hline Change & -0.01 & \pm & 0.11 & 0.01 & \pm & 0.14 \\
\hline ApoA-I (g/L) & & & & & \pm & \\
\hline Run in & 1.69 & \pm & 0.26 & 1.78 & \pm & 0.28 \\
\hline End & 1.63 & \pm & 0.27 & 1.71 & \pm & 0.31 \\
\hline Change & -0.06 & \pm & 0.07 & -0.07 & \pm & 0.15 \\
\hline \multicolumn{7}{|l|}{ TAG (mmol/L) } \\
\hline Run in & 0.92 & \pm & 0.61 & 1.06 & \pm & 0.82 \\
\hline End & 0.86 & \pm & 0.48 & 1.04 & \pm & 0.83 \\
\hline Change & -0.06 & \pm & 0.27 & -0.02 & \pm & 0.26 \\
\hline
\end{tabular}

Values are means $\pm S D$

Sterols and stanols concentrations are expressed as $10^{2} \times \mu \mathrm{mol} / \mathrm{mmol}$ cholesterol

$P$-values have been adjusted for the run-in period 


\section{References}

1. Gylling, H., et al., Plant sterols and plant stanols in the management of dyslipidaemia and prevention of cardiovascular disease. Atherosclerosis, 2014. 232(2): p. 346-60.

2. Naumann, E., et al., The baseline serum lipoprotein profile is related to plant stanol induced changes in serum lipoprotein cholesterol and triacylglycerol concentrations. J Am Coll Nutr, 2008. 27(1): p. 117-26.

3. Plat, J., et al., A plant stanol yogurt drink alone or combined with a low-dose statin lowers serum triacylglycerol and non-HDL cholesterol in metabolic syndrome patients. J Nutr, 2009. 139(6): p. 1143-9.

4. Demonty, I., et al., The effect of plant sterols on serum triglyceride concentrations is dependent on baseline concentrations: a pooled analysis of 12 randomised controlled trials. Eur J Nutr, 2013. 52(1): p. 153-60.

5. Plat, J. and R.P. Mensink, Plant stanol esters lower serum triacylglycerol concentrations via a reduced hepatic VLDL-1 production. Lipids, 2009. 44(12): p. 1149-53.

6. Schonewille, M., et al., Serum TG-lowering properties of plant sterols and stanols are associated with decreased hepatic VLDL secretion. J Lipid Res, 2014. 55(12): p. 2554-61.

7. Rideout, T.C., S.V. Harding, and P.J. Jones, Consumption of plant sterols reduces plasma and hepatic triglycerides and modulates the expression of lipid regulatory genes and de novo lipogenesis in C57BL/6J mice. Mol Nutr Food Res, 2010. 54 Suppl 1: p. S7-13.

8. Plat, J. and R.P. Mensink, Vegetable oil based versus wood based stanol ester mixtures effects on serum lipids and hemostatic factors in non-hypercholesterolemic subjects. Atherosclerosis, 2000. 148(1): p. 101-12.

9. de Jong, A., et al., Effects of long-term plant sterol or stanol ester consumption on lipid and lipoprotein metabolism in subjects on statin treatment. Br J Nutr, 2008. 100(5): p. 937-41.

10. Paiva, H., et al., High-dose statins and skeletal muscle metabolism in humans: a randomized, controlled trial. Clin Pharmacol Ther, 2005. 78(1): p. 60-8.

11. Khera, A.V., et al., Cholesterol efflux capacity, high-density lipoprotein function, and atherosclerosis. N Engl J Med, 2011. 364(2): p. 127-35.

12. Ritsch, A., et al., Phage-displayed recombinant single-chain antibody fragments with high affinity for cholesteryl ester transfer protein (CETP): cDNA cloning, characterization and CETP quantification. Clin Chem Lab Med, 2004. 42(3): p. 247-55.

13. Ritsch, A., et al., Enhancement of cholesteryl ester transfer in plasma by hormonereplacement therapy. Metabolism, 2002. 51(5): p. 599-604.

14. Kaser, S., et al., Lipoprotein profile and cholesteryl ester transfer protein in neonates. Metabolism, 2001. 50(6): p. 723-8.

15. Morgan, S.A., K. O'Dea, and A.J. Sinclair, A low-fat diet supplemented with monounsaturated fat results in less HDL-C lowering than a very-low-fat diet. J Am Diet Assoc, 1997. 97(2): p. 151-6.

16. Kesaniemi, Y.A. and S.M. Grundy, Turnover of low density lipoproteins during inhibition of cholesterol absorption by neomycin. Arteriosclerosis, 1984. 4(1): p. 41-8.

17. Katan, M.B., et al., Existence of consistent hypo- and hyperresponders to dietary cholesterol in man. Am J Epidemiol, 1986. 123(2): p. 221-34. 
18. Kalogirou, M., et al., Effect of ezetimibe monotherapy on the concentration of lipoprotein subfractions in patients with primary dyslipidaemia. Curr Med Res Opin, 2007. 23(5): p. 1169-76.

19. Homma, Y., et al., Decrease in plasma low-density lipoprotein cholesterol, apolipoprotein $B$, cholesteryl ester transfer protein, and oxidized low-density lipoprotein by plant stanol ester-containing spread. Nutrition, 2003. 19(4): p. 369-374.

20. Luo, Y. and A.R. Tall, Sterol upregulation of human CETP expression in vitro and in transgenic mice by an LXR element. J Clin Invest, 2000. 105(4): p. 513-20.

21. Honzumi, S., et al., LXRalpha regulates human CETP expression in vitro and in transgenic mice. Atherosclerosis, 2010. 212(1): p. 139-45.

22. Plat, J., J.A. Nichols, and R.P. Mensink, Plant sterols and stanols: effects on mixed micellar composition and LXR (target gene) activation. J Lipid Res, 2005. 46(11): p. 2468-76.

23. Foger, B., et al., Relationship of plasma cholesteryl ester transfer protein to HDL cholesterol. Studies in normotriglyceridemia and moderate hypertriglyceridemia. Arterioscler Thromb Vasc Biol, 1996. 16(12): p. 1430-6.

24. Miettinen, T.A. and Y.A. Kesaniemi, Cholesterol absorption: regulation of cholesterol synthesis and elimination and within-population variations of serum cholesterol levels. Am J Clin Nutr, 1989. 49(4): p. 629-35.

25. Nunes, V.S., et al., HDL-C concentration is related to markers of absorption and of cholesterol synthesis: Study in subjects with low vs. high HDL-C. Clin Chim Acta, 2011. 412(1-2): p. 176-80.

26. Lupattelli, G., et al., Non-cholesterol sterols in different forms of primary hyperlipemias. Nutr Metab Cardiovasc Dis, 2012. 22(3): p. 231-6.

27. Tilvis, R.S. and T.A. Miettinen, Serum plant sterols and their relation to cholesterol absorption. Am J Clin Nutr, 1986. 43(1): p. 92-7.

28. Eren, E., N. Yilmaz, and O. Aydin, High Density Lipoprotein and it's Dysfunction. Open Biochem J, 2012. 6: p. 78-93.

29. Camont, L., et al., Small, dense high-density lipoprotein-3 particles are enriched in negatively charged phospholipids: relevance to cellular cholesterol efflux, antioxidative, antithrombotic, anti-inflammatory, and antiapoptotic functionalities. Arterioscler Thromb Vasc Biol, 2013. 33(12): p. 2715-23.

30. Niesor, E.J., et al., Effect of dalcetrapib, a CETP modulator, on non-cholesterol sterol markers of cholesterol homeostasis in healthy subjects. Atherosclerosis, 2011. 219(2): p. 761-7.

31. Ray, K.K., et al., The effect of cholesteryl ester transfer protein inhibition on lipids, lipoproteins, and markers of HDL function after an acute coronary syndrome: the dalACUTE randomized trial. Eur Heart J, 2014. 35(27): p. 1792-800.

32. Nicholls, S.J., et al., Assessment of the clinical effects of cholesteryl ester transfer protein inhibition with evacetrapib in patients at high-risk for vascular outcomes: Rationale and design of the ACCELERATE trial. Am Heart J, 2015. 170(6): p. 1061-9.

33. van Capelleveen, J.C., et al., Effects of the cholesteryl ester transfer protein inhibitor, TA8995, on cholesterol efflux capacity and high-density lipoprotein particle subclasses. J Clin Lipidol, 2016. 10(5): p. 1137-1144.e3. 


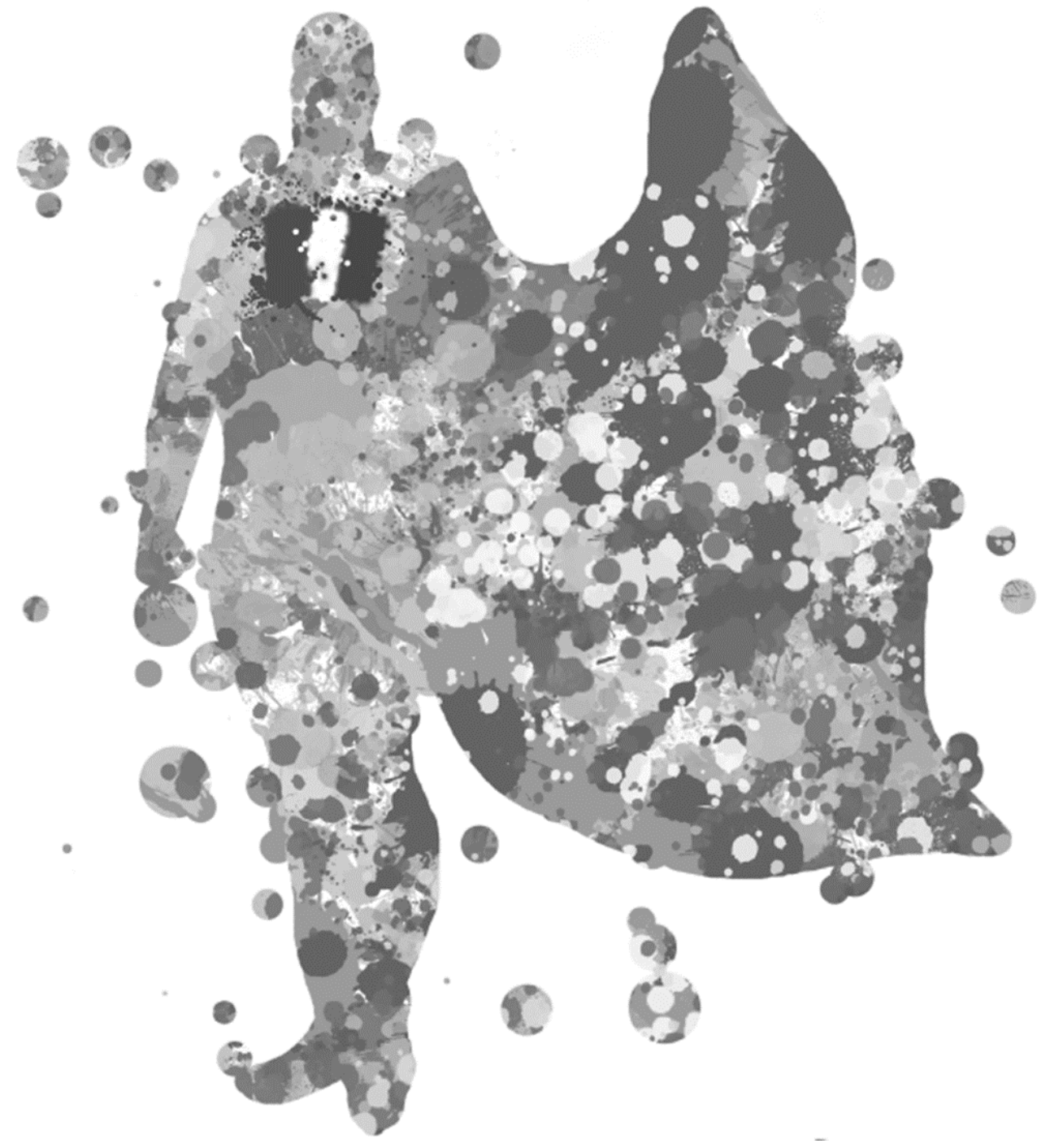




\section{Chapter 6}

\section{HDL cholesterol efflux capacity}

and CETP activity are associated with body mass, but are not

changed by diet-induced weight loss: a randomized trial in abdominally obese men

Charlotte P.J. Talbot, Jogchum Plat, Peter J. Joris, Maurice Konings, Yvo H.A.M. Kusters, Casper G. Schalkwijk, Andreas Ritsch, Ronald P. Mensink 


\begin{abstract}
Background and Aims: Obesity is associated with a lower HDL-mediated cholesterol efflux from macrophages and a higher CETP (cholesteryl ester transfer protein) activity, but effects of weight loss are not clear. Also, associations with visceral and subcutaneous adipose tissue are not known. We therefore investigated effects of diet-induced weight loss on HDL-mediated cholesterol efflux and cholesterol ester (CE) transfer in abdominally obese men. Differences between normal-weight and abdominally obese men were also examined.
\end{abstract}

Methods: Twenty-five apparently healthy, normal-weight men (waist circumference: $<94 \mathrm{~cm}$ ) and 52 abdominally obese men (waist circumference: 102 $110 \mathrm{~cm}$ ) were included. Abdominally obese subjects were randomly allocated to a dietary weight-loss intervention group or a no-weight loss control group. Individuals from the intervention group followed a very-low-calorie diet for 6 weeks to obtain a waist circumference below $102 \mathrm{~cm}$, followed by a 2-weeks weight-stable period. Cholesterol efflux was measured in BODIPY-labeled murine J774 macrophages. $\mathrm{CE}$ transfer was measured by quantifying the transfer of $\mathrm{CE}$ from radiolabeled exogenous HDL to apoB-containing lipoproteins.

Results: Cholesterol efflux capacity was 9 percentage point (pp) lower in abdominally obese than in normal-weight men $(p \leq 0.001)$, while $C E$ transfer was 5 $\mathrm{pp}$ higher $(\mathrm{p} \leq 0.01)$. Diet-induced weight-loss of $10.3 \mathrm{~kg}$ did not change cholesterol efflux and CE transfer. In addition, stepwise regression analysis did not suggest that the different fat depots are differently related to efflux capacity and CE transfer.

Conclusions: After a 2-week weight-stable period, dietary weight loss of $10 \mathrm{~kg}$ did not improve ABCA1-mediated cholesterol efflux and CE transfer in abdominally obese men. 


\section{Introduction}

The role of high-density lipoproteins (HDL) in the prevention of coronary heart disease (CHD) is not clear, as raising HDL-cholesterol (HDL-C) concentrations per se is not causally related to a lower risk for CHD [1,2]. However, it becomes more and more accepted that improving HDL functionality is a better target to reduce CHD risk. HDL has many anti-atherogenic properties, including anti-inflammatory, anti-oxidant, anti-apoptotic, anti-thrombotic and vasodilating effects [3]. However, its involvement in the reverse cholesterol transport (RCT) pathway, which is the ability of HDL to remove and transport the excess of cholesterol from peripheral cells such as residing macrophages in the arterial wall to the liver for excretion, represents its main anti-atherogenic effect [3,4]. Indeed, an inverse association between cholesterol efflux with atherosclerosis and cardiovascular mortality has been reported, independent of HDL-C concentrations [5, 6].

HDL-mediated cholesterol efflux from macrophages is facilitated by several transporters, such as ABCA1, ABCG1 and SR-BI [3], and related to numerous factors, including HDL particles size and composition as well as differences in baseline characteristics between subjects. One of these factors is body fat. Although not all obese subjects have increased levels of inflammatory markers [7], obesity is frequently associated with chronic low-grade inflammation [8]. As proinflammatory cytokines reduce in vitro cholesterol efflux capacity [9, 10], abdominally obese subjects may even have a further lowered cholesterol efflux [11, $12]$, because visceral fat secretes more cytokines and adipokines than subcutaneous fat [8]. In addition, adipocytes may be involved in the transport of cholesterol to HDL particles, which is reduced in inflamed adipocytes [13]. Obese subjects also have an increased mass and activity of cholesteryl ester transfer protein (CETP) mediating the exchange of cholesteryl esters (CE) and triacylglycerol (TAG) between HDL and apoB100-containing lipoproteins [14]. However, not much is known about the relationship between cholesteryl ester (CE) transfer and cholesterol efflux, even though CE transfer is inversely associated with HDL particle size and composition $[15,16]$, which both play an important role in cholesterol efflux [17].

Although cross-sectional differences in efflux capacity between various BMI-groups have previously been reported $[11,12,18]$, the effects of diet-induced weight loss on cholesterol efflux capacity are less consistent. Some studies have reported a decrease in cholesterol efflux after weight loss [19, 20], while others have reported no effects [21]. Also, relationships with the different fat depots are not known. Finally, the reported studies were lacking a no-weight loss control group. Therefore, we measured in a randomized controlled trial the effects of diet-induced weight loss on HDL-mediated cholesterol efflux and CE transfer in abdominally obese men. Cross- 
sectional differences at baseline between normal-weight and abdominally obese men were also investigated.

\section{Materials and Methods}

\section{Subjects and Design}

To examine the effects of weight-loss on cholesterol efflux capacity of HDL, samples from a previous study were used. In that study, effects of diet-induced weight loss on cardiometabolic risk markers and vascular function markers in abdominally obese men (waist circumference between 102-110 cm) were examined. Details of this study have already been published [22, 23]. Briefly, baseline measurements were completed in 54 abdominally obese men. One man dropped out before randomization and 53 men were randomly assigned to the weight-loss or no-weight loss control group. One participant discontinued the control intervention because of illness, while another man dropped out because of non-compliance with the weightloss program. Another man from the weight-loss group stopped for personal reasons. Thus, eventually 50 abdominally obese men completed the entire weightloss study. At the end of the study, one participant was excluded from the analyses, because of unreported use of antihypertensive medication during the study. Therefore, data from 49 abdominally obese men were used for the present analysis. For comparison, a group of 25 age-matched normal-weight men (waist circumference $<94 \mathrm{~cm}$ ) was included. All 74 subjects were apparently healthy, aged between 18-65 years, and had a stable body weight (weight gain or loss $<3 \mathrm{~kg}$ within the previous 3 months). Use of medically prescribed drugs known to affect lipids, glucose and blood pressure were not allowed. The study was conducted according the ethical guidelines of the 1975 Declaration of Helsinki and approved by the Ethics Committee of Maastricht University Medical Centre. Written informed consent was obtained from all participants before entering the study. The study was registered at clinicaltrials.gov as NCT01675401.

Subjects assigned to the weight-loss program $(n=25)$ had to consume daily a verylow-calorie diet (VLCD; Modifast; Nutrition et Santé Benelux) providing 2.1 MJ (500 $\mathrm{kcal}$ ) and a maximum of $250 \mathrm{~g}$ vegetables or fruit (except bananas) each day for 4 weeks to reach a waist circumference below $102 \mathrm{~cm}$, the cut-off value for one of the criteria to diagnose of the metabolic syndrome [24]. If the waist circumference was not below $102 \mathrm{~cm}$ after 4 weeks, subjects had to consume the VLCD for another week, while the calorie-restricted diet was used for 1 week. After the VLCD period, subjects were prescribed a mixed-solid, calorie-restricted diet, which provided 4.2 $\mathrm{MJ} /$ day for 2 weeks, as a transition period. The composition of this diet was in agreement with the Dutch dietary guidelines. In weeks 7 and 8 , subjects consumed 
daily menus based on the new estimated energy requirements based on their newly achieved body weight in order to keep them weight stable for two weeks. Participants assigned to the no-weight loss control group $(n=27)$ were asked to maintain their habitual diet and use of alcohol throughout the total study period. In addition, all subjects were requested not to change their physical activity levels and to write down daily any protocol deviations in a diary. Diaries were checked at each visit and no deviations were noted that could have influenced the results. The 25 normal-weight participants were only measured once during the study and were not subjected to any intervention.

\section{Blood sampling}

Fasting blood samples were collected at the start and at the end of the intervention period. EDTA and $\mathrm{NaF}$ tubes were centrifuged at $1300 \times \mathrm{g}$ for $15 \mathrm{~min}$ at $4^{\circ} \mathrm{C}$ to obtain plasma. Serum samples were obtained after blood samples were centrifuged at 1300 $\times \mathrm{g}$ for $15 \mathrm{~min}$ at $21^{\circ} \mathrm{C}[22]$. The supernatants were transferred into $1.5 \mathrm{ml}$ Eppendorf tubes and stored immediately at $-80^{\circ} \mathrm{C}$ until further analysis.

\section{Measurements}

Subcutaneous and visceral adipose tissue volumes were measured as described [22, 23]. The percentage of body fat was assessed by taking skinfold measurements, using the Durnin and Womersley formula [25].

Serum samples were used to measure concentrations of HDL-C (CHOD/PAP method; Roche Diagnostics System, Hofmann-La Roche Ltd., Basel, Swiss), TAG (GPO-Trinder; Sigma Diagnostics, St Louis, USA), apoA-I and apolipoprotein B100 (apoB100) (Horiba ABX, Montpellier Cedex, France), insulin, and C-peptide (RIA, Millipore, Linco Research, Missouri, USA). NaF plasma samples were used for glucose measurements (Horiba ABX, Montpellier Cedex, France). The HOMA-IR was calculated to estimate the degree of insulin resistance [26]. EDTA plasma samples were used to measure high-sensitive C-reactive protein concentrations (hsCRP) by using a multiarray detection system based on electrochemiluminescence technology (SECTOR Imager 2400; Meso Scale Discovery). For all analyses, all samples from one subject were analyzed within the same run.

The ability of HDL particles to promote cholesterol efflux from macrophages was measured as described previously [27]. Briefly, murine J774 macrophages were first cultivated and incubated overnight with $14.42 \mu \mathrm{g} / \mathrm{mL}$ BODIPY-cholesterol, i.e. 50 000 cells were seeded in a total incubation volume of $150 \mu \mathrm{l}$ in a 96-well plate. Next, macrophages were equilibrated for $4 \mathrm{~h}$ with $1 \mu \mathrm{l} / \mathrm{ml}$ of an ACAT inhibitor (Acyl CoA 
Acyltransferase Inhibitor (Sandoz 58-035) purchased from Sigma-Aldrich) and activated overnight with $4 \mu \mathrm{l} / \mathrm{ml}$ cAMP to upregulate the expression of the cholesterol-efflux transporter ABCA1. Thus, this efflux assay used mainly measures the ABCA1-mediated cholesterol efflux from macrophages to its major acceptor pre $\beta$ - 1 HDL [3]. Next, in plasma samples from the volunteers in the study, apolipoprotein B containing lipoproteins were precipitated using tungstophosphoric acid hydrate and magnesium chloride. After spinning, the supernatant containing the HDL particles was added to the labeled macrophages and incubated for $4 \mathrm{~h}$. All steps were performed in the presence of $1 \mu \mathrm{l} / \mathrm{ml}$ of ACAT inhibitor to block cholesterol esterification. After $4 \mathrm{~h}$ incubation, the $96-$ well plates were centrifuged for 5 minutes at $800 \mathrm{rpm}$ at room temperature, and the supernatant of each sample was pipetted into a Fluoro Nunc plate (236105, Thermo Scientific, Waltham, Massachusetts, USA). The fluorescence intensity from each sample was quantified using the Tecan Infinite 200 plate reader (excitation $482 \mathrm{~nm}$, emission 515 $\mathrm{nm}$ ). Serum controls from two healthy subjects were included in each assay as reference. The efflux capacity values for each subject were expressed relative to the average efflux capacity value of these two serum pools, which was set to $100 \%$. Each sample was analyzed in triplicate.

Cholesteryl ester (CE) transfer measurements were quantified by determining the transfer of CE from radiolabeled exogenous HDL to apoB-containing lipoproteins. As described [28, 29], HDL particles from plasma samples were isolated by ultracentrifugation, and radiolabeled with ${ }^{3} \mathrm{H}$-cholesterol. Whole serum from each participant was then incubated with the radiolabeled HDL. After incubation, apoBcontaining lipoproteins were precipitated with dextranesulphate/ $\mathrm{MgCl}_{2}$. After lowspeed centrifugation, the supernatant was transferred into a scintillation vial and the radioactivity was determined using a beta counter. Lipoprotein deficient plasma was used as negative control. CE transfer was measured as the percentage of total radiolabeled $\mathrm{CE}$ transferred to apoB-containing lipoproteins, normalized to the initial amount of radioactivity present in the supernatant (3000 counts per minute). Thus, the higher the percentage of radioactivity in the supernatant, the lower the percentage of $\mathrm{CE}$ transferred to the apoB-containing lipoproteins pellet.

\section{Statistics}

Results are presented as means \pm standard deviations, unless otherwise indicated. Cross-sectional differences at baseline between normal-weight and abdominally obese men were analyzed by an independent-samples t-test. Differences in changes between the groups, i.e. diet-induced weight-loss and no-weight loss control treatments, were tested using the one-way ANCOVA with adjustment for baseline 
values. Pearson correlation coefficients were used to examine cross-sectional relations between cholesterol efflux and CE transfer with anthropometric measurements and cardiometabolic risk markers (age; BMI; waist-to-hip ratio, visceral, subcutaneous and total fat; concentrations of HDL-C, apoA-I, apoB100, TAG, CRP, glucose, C-peptide and insulin; and HOMA-IR). Because the CRP data were not normally distributed, as assessed with the Shapiro-Wilk test, Spearman's rho coefficient was used to examine correlations. Using stepwise regression analysis, the independent contributions of the cardiometabolic risk markers on plasma cholesterol efflux capacity and CE transfer was examined. Only parameters that correlated significantly were initially entered into the model. A P value $\leq 0.05$ was considered significant. All statistical analyses were performed with SPSS 20.0 for Mac OS.

\section{Results}

\section{Baseline characteristics of the study population}

Baseline characteristics of the 25 normal-weight and 52 abdominally obese men are presented in Supplemental Table I. Age was comparable between the normalweight and abdominally obese men. As expected, BMI, visceral and subcutaneous fat, and total fat mass were significantly higher in the obese men. Further, fasting serum concentrations of HDL-C were 0.15 percentage point $(\mathrm{pp})$ lower in the abdominally obese men ( $\mathrm{p} \leq 0.01)$, while those of apoB100, TAG and CRP were higher ( $p<0.05$ for all variables). Cholesterol efflux capacity was $9 \mathrm{pp}$ lower in obese men, whereas $C E$ transfer was $5 \mathrm{pp}$ higher ( $\mathrm{p} \leq 0.01$ for both variables). Finally, serum apoA1 concentrations were comparable between the normal-weight and abdominally obese men.

\section{Effect of the weight-loss intervention}

Forty-nine abdominally obese subjects completed the intervention study. In the 23 subjects in the weight loss group, body weight decreased on average by $10.3 \mathrm{~kg}$, BMI by $3.1 \mathrm{~kg} / \mathrm{m}^{2}$, visceral fat by $0.85 \mathrm{~L}$, subcutaneous fat by $0.84 \mathrm{~L}$, and fat mass by 4.4 pp, compared with the control group [22,23]. As planned, during the last two weeks of the study, body weight was stable [22]. Weight loss did not change serum HDL$\mathrm{C}$ concentrations, while apoA-I concentrations were significantly decreased after weight-loss by $0.07 \mathrm{~g} / \mathrm{L}$ ( $95 \% \mathrm{CI}:-0.14,-0.01 \mathrm{~g} / \mathrm{L} ; \mathrm{p}=0.03)$. ApoB100 concentrations were also decreased by $0.17 \mathrm{~g} / \mathrm{L}(95 \% \mathrm{CI}:-0.24,-0.10 \mathrm{~g} / \mathrm{L} ; \mathrm{p}=0.04)$. However, cholesterol efflux and CE transfer did not change significantly (Table 1). 


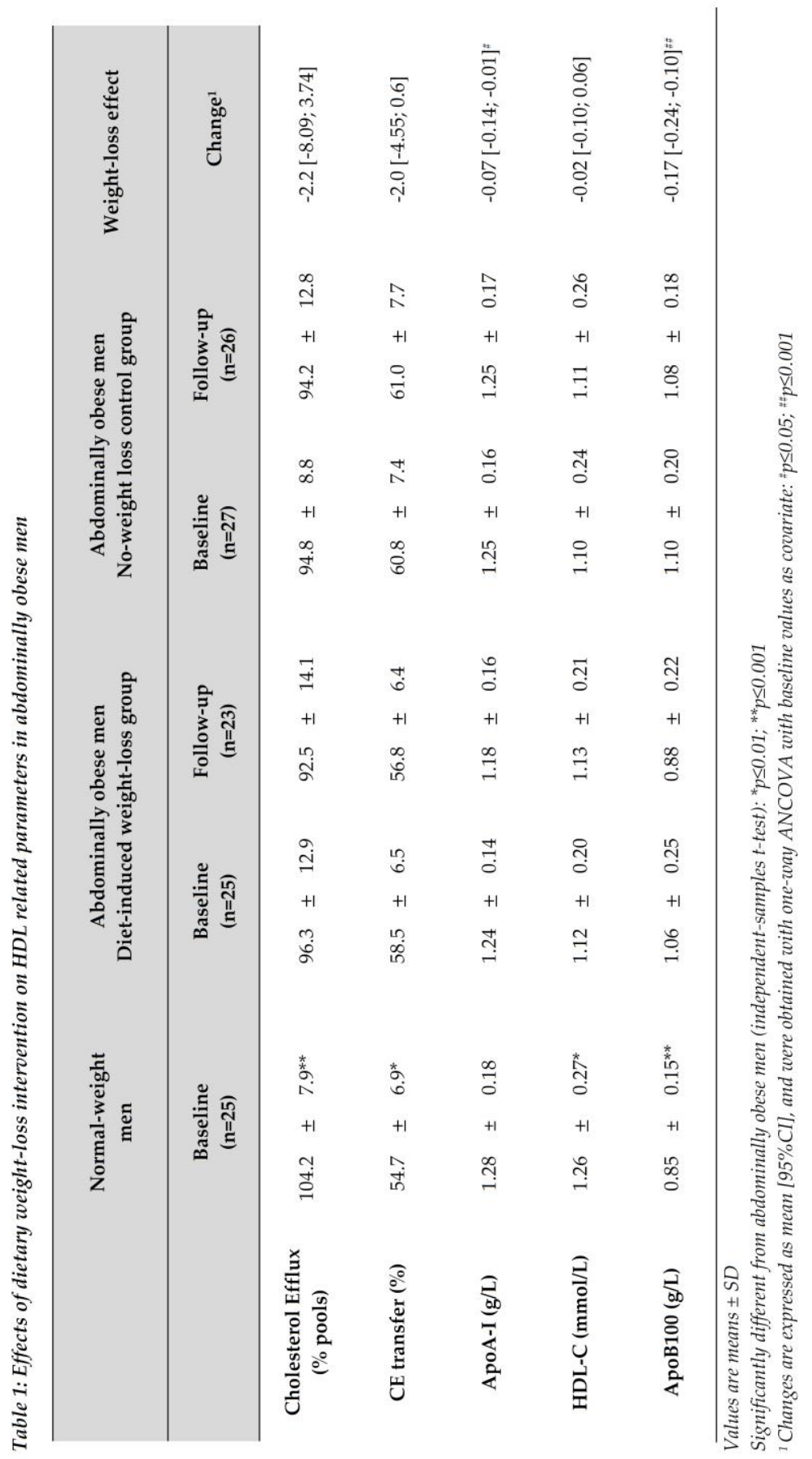




\section{Correlations between HDL parameters}

At baseline, cholesterol efflux capacity was significantly associated with BMI ( $\mathrm{r}=-$ $0.349 ; \mathrm{p}<0.01 ; \mathrm{n}=77)$, as well as with visceral, subcutaneous and total fat $[(\mathrm{r}=-0.285$; $\mathrm{p} \leq 0.01 ; \mathrm{n}=76),(\mathrm{r}=-0.340 ; \mathrm{p}<0.01 ; \mathrm{n}=76),(\mathrm{r}=-0.302 ; \mathrm{p}<0.01 ; \mathrm{n}=77)$, respectively]. Finally, CRP concentrations showed a negative correlation with cholesterol efflux capacity $(\mathrm{r}=-0.292 ; \mathrm{p} \leq 0.01 ; \mathrm{n}=77)$. Stepwise multivariate linear regression including the significant variables showed that only BMI remained as a significant predictor of cholesterol efflux capacity $(\beta=-1.1 ; \mathrm{p} \leq 0.001)$.

CE transfer at baseline was associated with BMI $(\mathrm{r}=0.312 ; \mathrm{p}<0.01 ; \mathrm{n}=77)$, the waist-tohip ratio $(\mathrm{r}=0.365 ; \mathrm{p} \leq 0.001)$, and concentrations of HDL-C $(\mathrm{r}=-0.473 ; \mathrm{p}<0.001 ; \mathrm{n}=77)$, apoA-I ( $\mathrm{r}=-0.267 ; \mathrm{p}<0.05 ; \mathrm{n}=77)$, apoB100 ( $\mathrm{r}=0.580 ; \mathrm{p}<0.001 ; \mathrm{n}=77)$, TAG $(\mathrm{r}=0.430$; $\mathrm{p}<0.001 ; \mathrm{n}=77)$ and CRP $(\mathrm{r}=0.260 ; \mathrm{p}<0.05 ; \mathrm{n}=77)$. Moreover, CE transfer showed a significant positive association with $\mathrm{C}$-peptide, insulin concentrations and the HOMA-IR ( $r=0.475 ; r=0.450$ and $r=0.440$, respectively; $p<0.001$ for all three variables). Stepwise linear regression analysis including these significant variables showed that HDL-C $(\beta=-11.9 ; p<0.001)$ and apoB100 $(\beta=16.5 ; p<0.001)$ predicted $C E$ transfer.

\section{Discussion}

In this study, we found that ABCA1-mediated cholesterol efflux from cholesterolloaded macrophages to HDL is higher in normal-weight subjects than in abdominally obese men. However, diet-induced weight loss in the abdominally obese men did not improve cholesterol efflux. Comparable results were observed for CE transfer and HDL-C concentrations, while apoA-I concentrations were even decreased after weight loss.

So far, only a few other studies addressed the effect of dietary induced weight loss on HDL-mediated cholesterol efflux. In these studies, however, no-weight loss control groups were not included, while results were highly variable. Weight-loss of $25 \mathrm{~kg}$ induced by a VLCD within a period of 16 weeks reduced cholesterol-efflux, while HDL-C concentrations were not changed and apoA-I concentrations were increased [19]. In another study, weight loss of nearly $10 \mathrm{~kg}$ after 4 to 6 weeks reduced ABCA1- and ABCG1-mediated cholesterol efflux to HDL. Like in our study, HDL-C concentrations were not changed, while apoA-I concentrations were decreased [20]. In a third study with overweight or obese women, a decrease of about $2 \mathrm{~kg}$ after 6 months induced by caloric restriction plus low-intensity exercise, 
decreased HDL-C concentrations, while apoA-I concentrations as well as ABCG1and SR-BI-mediated efflux pathways were not changed. Surprisingly, ABCA1mediated efflux was decreased, although the exercise regime might also have influenced the results [21]. In three other studies, effects of surgery-induced weight loss were examined. Weight loss in these studies was of course larger and were, respectively, $20 \mathrm{~kg}$ [30], $25 \mathrm{~kg}$ [31] and $56 \mathrm{~kg}$ [32]. Despite increases in HDL-C in all three studies, effects on cholesterol efflux - or the transporters involved - were not consistent. In one study, SR-BI- and ABCG1-mediated cholesterol efflux was increased, while the ABCA1-mediated cholesterol efflux was decreased [30]. In contrast, cholesterol efflux was increased after upregulation of ABCA1 expression in the two other studies [31,32]. No clear reason for these discrepant findings can be provided. One possible explanation may relate to study duration. It has been suggested that HDL-C concentrations increase after weight-loss, but only during the subsequent weight-stable period, whereas they are even decreased during the active weight loss period [33]. In our study, subjects were weight stable for 2 weeks, which may have been too short for HDL metabolism to reach a new steady state. In the other diet-induced weight-loss studies [19-21], a weight stable period was however not included at all. Therefore, it would be of interest to examine effects on HDL metabolism after a longer-weight stable period. Another explanation may relate to the amount of weight loss, which was by far larger after surgery than after dietinduced weight loss. In addition, differences in metabolic disturbances may have played a role. Although our population was abdominally obese, they had no clinical manifestations of any disease. In contrast, in other studies effects of weight loss were examined in insulin-dependent type 2 diabetic patients [19], in patients with the metabolic syndrome [20], or in overweight women, of which $12 \%$ were diabetic [21]. Finally, changes in HDL subpopulations following weight loss might also play a role. Indeed, weight reduction is associated with an increase in HDL2 particle concentrations and a decrease in HDL3 particle concentrations [34]. In addition, the increase in HDL2 following surgery-induced weight loss was associated with an improvement in both ABCG1- and SR-BI-efflux pathways. In contrast, ABCA1mediated efflux was decreased, suggesting that pre $\beta-1$ HDL concentration was decreased by weight loss [30]. Possibly, weight loss has a greater effect on the ABCG1 and SR-BI-mediated efflux pathways than on the ABCA1-mediated efflux. In addition, when using apoB-depleted serum as cholesterol acceptor, not only (pre $\beta-1)$ HDL, but also other factors such as albumin, may contribute to the cholesterol efflux [35] However, it should be noted that with our assay we did observe cross-sectional differences in cholesterol efflux between the abdominally obese and the normal-weight men at baseline. This suggests that ABCA1-mediated cholesterol efflux is related to body mass. 
To the best of our knowledge, the relationship between fat distribution and cholesterol efflux capacity has not been investigated so far. Visceral fat is thought to secrete more pro-inflammatory cytokines than subcutaneous fat [8]. As inflammation is negatively associated with cholesterol efflux capacity [36], this could theoretically explain the lower cholesterol efflux capacity in obese subjects. In fact, our lean subjects also had lower CRP concentrations than the abdominally obese subjects. However, CRP levels were not changed after the weight loss intervention. It has been estimated that for each $1 \mathrm{~kg}$ of weight loss, CRP levels decreases with $0.13 \mathrm{mg} / \mathrm{L}$ [37]. Possibly, the amount of weight loss in our study was too small to observe any statistically significant effect [22].

In contrast to other studies [38-40], no association between cholesterol efflux and the waist-to-hip ratio was found. Efflux capacity was negatively associated with both visceral and subcutaneous fat, but after stepwise linear regression analysis, only BMI remained a significant predictor of cholesterol efflux capacity. As all the abdominally obese men a waist circumference between $102-110 \mathrm{~cm}$, it is possible that the range in the waist-to-hip ratio was not large enough.

Reductions in CE transfer have been reported after surgery-induced weight loss [14, 30, 41]. However, not many studies have addressed the effects of diet-induced weight loss on CE transfer. In a small study with 4 women without control group, 2 months of calorie restriction was associated with a reduction in both CETP activity and mass [42]. In another study with obese men, the intake of a low-fat energyrestricted diet for 3 months did not change $\mathrm{CE}$ transfer, despite a $10 \mathrm{~kg}$ reduction in body weight [43]. A 4 weeks low caloric diet-induced weight loss decreased body weight by $4 \mathrm{~kg}$ and was associated with a decrease in CETP mass concentrations [44]. Finally, caloric restriction for 16 weeks was accompanied by a decrease in CETP concentration [19]. However, CE transfer was not investigated in these two latter studies $[19,44]$.

As in other studies [14, 42, 45, 46], BMI was positively associated with CE transfer, which might be related to the CETP synthesis in adipose tissue [47], as CETP mRNA levels in adipose tissue are positively associated with plasma CETP concentrations [48]. As CETP expression is higher in subcutaneous fat than in visceral fat [49], and the subcutaneous fat depot is larger, it can be expected that CETP mass is more strongly correlated with subcutaneous fat, as shown by Arai et al. [42]. However, these findings were not confirmed in a recent large cross-sectional study examining associations between fat distribution and CETP concentrations [50]. Although CETP activity does not necessarily reflect CETP concentrations [51], we also found no evidence that the various fat depots were differently associated with CETP activity. 
The relationship between CE transfer and cholesterol efflux is not clear. In one study, elevated CE transfer was positively associated with cholesterol efflux capacity in women, but not in men [52]. In addition, in patients at risk for cardiovascular disease, a high plasma concentration of CETP was associated with a high efflux capacity, and CETP mass was found to significantly predict cholesterol efflux capacity [53]. Unfortunately, we did not measure CETP mass in the present study. However, no association between CE transfer and cholesterol efflux capacity was found.

In conclusion, the present study shows that in men ABCA1-mediated macrophage cholesterol efflux and CE transfer are associated with body mass. However, these parameters were not changed after a six weeks diet-induced weight loss of $10 \mathrm{~kg}$ in abdominally obese men followed by a 2 weeks weight-stable period. Future studies should address the importance of a longer period of stable body weight and the involvement of other transporters, such as ABCG1 and SR-BI, on cholesterol efflux from macrophages. 


\section{Supplementary data}

Table 1: Baseline characteristics of the normal-weight and abdominally obese men

\begin{tabular}{|c|c|c|c|c|c|c|}
\hline & \multicolumn{3}{|c|}{$\begin{array}{l}\text { Normal-weight men } \\
(\mathrm{n}=25)\end{array}$} & \multicolumn{3}{|c|}{$\begin{array}{l}\text { Abdominally obese men } \\
\qquad(\mathrm{n}=52)\end{array}$} \\
\hline Age (years) ${ }^{1}$ & 53.7 & & 9-61.6] & 51.8 & & $-60.7]$ \\
\hline BMI & 23.3 & \pm & 1.8 & 30.1 & \pm & $2.1^{* * *}$ \\
\hline Visceral fat $(\mathrm{L})^{2}$ & 0.90 & \pm & 0.42 & 2.34 & \pm & $0.71^{* * *}$ \\
\hline Subcutaneous fat $(\mathrm{L})^{2}$ & 1.45 & \pm & 0.51 & 3.09 & \pm & $0.77^{* * *}$ \\
\hline Fat mass $(\%)$ & 18.5 & \pm & 5.4 & 28.2 & \pm & $3.5^{* * *}$ \\
\hline HDL-C (mmol/L) & 1.26 & \pm & 0.27 & 1.11 & \pm & $0.22^{* *}$ \\
\hline ApoA-I (g/L) & 1.28 & \pm & 0.18 & 1.24 & \pm & 0.15 \\
\hline $\operatorname{ApoB100}(g / L)$ & 0.85 & \pm & 0.15 & 1.08 & \pm & $0.23^{* * *}$ \\
\hline Triacylglycerol (mmol/L) & 1.00 & \pm & 0.48 & 1.81 & \pm & $0.93^{* * *}$ \\
\hline Glucose (mmol/L) & 5.17 & \pm & 0.28 & 5.48 & \pm & $0.52^{* *}$ \\
\hline C-peptide (ng/mL) & 0.95 & \pm & 0.21 & 1.69 & \pm & $0.67^{* * *}$ \\
\hline Insulin $(\mathrm{uU} / \mathrm{mL})$ & 7.1 & \pm & 1.8 & 11.5 & \pm & $5.4^{* * *}$ \\
\hline HOMA-IR & 1.6 & \pm & 0.5 & 2.8 & \pm & $1.4^{* * *}$ \\
\hline $\operatorname{CRP}(\mu \mathrm{g} / \mathrm{mL})^{1}$ & 0.61 & {$[0.3$} & $2-1.21]$ & 1.49 & {$[0.5$} & $-3.22]^{*}$ \\
\hline Cholesterol Efflux (\% pools) & 104.2 & \pm & 7.9 & 95.5 & \pm & $10.9^{* * *}$ \\
\hline CE transfer (\% transfer) & 54.7 & \pm & 6.9 & 59.7 & \pm & $7.1^{* *}$ \\
\hline
\end{tabular}

Values are means $\pm S D$

${ }^{1}$ Values are medians $\left[25-75^{\text {th }}\right.$ percentile]

${ }^{2}$ Normal-weight subjects: $n=24$

Significantly different from the normal-weight men (independent-samples t-test): ${ }^{*} \leq 0.05 ;{ }^{* *} p \leq 0.01 ;{ }^{* * *} p \leq 0.001$ 


\section{References}

1. Barter, P., et al., High density lipoproteins (HDLs) and atherosclerosis; the unanswered questions. Atherosclerosis, 2003. 168(2): p. 195-211.

2. Choi, B.G., et al., The role of high-density lipoprotein cholesterol in the prevention and possible treatment of cardiovascular diseases. Curr Mol Med, 2006. 6(5): p. 571-87.

3. Tall, A.R., Cholesterol efflux pathways and other potential mechanisms involved in the athero-protective effect of high density lipoproteins. J Intern Med, 2008. 263(3): p. 256-73.

4. Phillips, M.C., Molecular mechanisms of cellular cholesterol efflux. J Biol Chem, 2014. 289(35): p. 24020-9.

5. Khera, A.V., et al., Cholesterol efflux capacity, high-density lipoprotein function, and atherosclerosis. N Engl J Med, 2011. 364(2): p. 127-35.

6. Ritsch, A., H. Scharnagl, and W. Marz, HDL cholesterol efflux capacity and cardiovascular events. N Engl J Med, 2015. 372(19): p. 1870-1.

7. Alam, I., T.P. Ng, and A. Larbi, Does inflammation determine whether obesity is metabolically healthy or unhealthy? The aging perspective. Mediators Inflamm, 2012. 2012: p. 456456.

8. Wang, Z. and T. Nakayama, Inflammation, a link between obesity and cardiovascular disease. Mediators Inflamm, 2010. 2010: p. 535918.

9. Wang, X., et al., C-reactive protein inhibits cholesterol efflux from human macrophagederived foam cells. Arterioscler Thromb Vasc Biol, 2008. 28(3): p. 519-26.

10. Field, F.J., K. Watt, and S.N. Mathur, TNF-alpha decreases ABCA1 expression and attenuates HDL cholesterol efflux in the human intestinal cell line Caco-2. J Lipid Res, 2010. 51(6): p. 1407-15.

11. Sasahara, T., et al., Cholesterol transport between cells and high density lipoprotein subfractions from obese and lean subjects. J Lipid Res, 1998. 39(3): p. 544-54.

12. Autran, D., et al., Basal and postprandial serum-promoted cholesterol efflux in normolipidemic subjects: Importance of fat mass distribution. Metabolism, 2001. 50(11): p. 1330-5.

13. Zhang, Y., et al., Adipocyte modulation of high-density lipoprotein cholesterol. Circulation, 2010. 121(11): p. 1347-55.

14. Ebenbichler, C.F., et al., Relationship between cholesteryl ester transfer protein and atherogenic lipoprotein profile in morbidly obese women. Arterioscler Thromb Vasc Biol, 2002. 22(9): p. 1465-9.

15. Lagrost, L., et al., Influence of plasma cholesteryl ester transfer activity on the LDL and HDL distribution profiles in normolipidemic subjects. Arterioscler Thromb, 1993. 13(6): p. 815-25.

16. Huesca-Gomez, C., et al., Contribution of cholesteryl ester transfer protein and lecithin:cholesterol acyltransferase to HDL size distribution. Endocr Res, 2004. 30(3): p. 403-15.

17. Camont, L., M.J. Chapman, and A. Kontush, Biological activities of HDL subpopulations and their relevance to cardiovascular disease. Trends Mol Med, 2011. 17(10): p. 594-603. 
18. Attia, N., et al., Impact of android overweight or obesity and insulin resistance on basal and postprandial SR-BI and ABCA1-mediated serum cholesterol efflux capacities. Atherosclerosis, 2010. 209(2): p. 422-9.

19. Wang, Y., et al., Prolonged caloric restriction in obese patients with type 2 diabetes mellitus decreases plasma CETP and increases apolipoprotein AI levels without improving the cholesterol efflux properties of HDL. Diabetes Care, 2011. 34(12): p. 2576-80.

20. Vasudevan, M., et al., Modest diet-induced weight loss reduces macrophage cholesterol efflux to plasma of patients with metabolic syndrome. J Clin Lipidol, 2013. 7(6): p. 661-70.

21. Aicher, B.O., et al., Diet-induced weight loss in overweight or obese women and changes in high-density lipoprotein levels and function. Obesity (Silver Spring), 2012. 20(10): p. 2057-62.

22. Joris, P.J., et al., Diet-induced weight loss improves not only cardiometabolic risk markers but also markers of vascular function: a randomized controlled trial in abdominally obese men. Am J Clin Nutr, 2017. 105(1): p. 23-31.

23. Kusters, Y.H., et al., Independent tissue contributors to obesity-associated insulin resistance. JCI Insight, 2017. 2(13).

24. Third Report of the National Cholesterol Education Program (NCEP) Expert Panel on Detection, Evaluation, and Treatment of High Blood Cholesterol in Adults (Adult Treatment Panel III) final report. Circulation, 2002. 106(25): p. 3143-421.

25. Durnin, J.V. and J. Womersley, Body fat assessed from total body density and its estimation from skinfold thickness: measurements on 481 men and women aged from 16 to 72 years. $\mathrm{Br}$ J Nutr, 1974. 32(1): p. 77-97.

26. Matthews, D.R., et al., Homeostasis model assessment: insulin resistance and beta-cell function from fasting plasma glucose and insulin concentrations in man. Diabetologia, 1985. 28(7): p. 412-9.

27. Sankaranarayanan, S., et al., A sensitive assay for ABCA1-mediated cholesterol efflux using BODIPY-cholesterol. J Lipid Res, 2011. 52(12): p. 2332-40.

28. Ritsch, A., et al., Enhancement of cholesteryl ester transfer in plasma by hormonereplacement therapy. Metabolism, 2002. 51(5): p. 599-604.

29. Kaser, S., et al., Lipoprotein profile and cholesteryl ester transfer protein in neonates. Metabolism, 2001. 50(6): p. 723-8.

30. Aron-Wisnewsky, J., et al., Effect of bariatric surgery-induced weight loss on SR-BI-, ABCG1-, and ABCA1-mediated cellular cholesterol efflux in obese women. J Clin Endocrinol Metab, 2011. 96(4): p. 1151-9.

31. Osto, E., et al., Rapid and body weight-independent improvement of endothelial and highdensity lipoprotein function after Roux-en-Y gastric bypass: role of glucagon-like peptide-1. Circulation, 2015. 131(10): p. 871-81.

32. Davidson, W.S., et al., Weight loss surgery in adolescents corrects high-density lipoprotein subspecies and their function. Int J Obes (Lond), 2017. 41(1): p. 83-89.

33. Dattilo, A.M. and P.M. Kris-Etherton, Effects of weight reduction on blood lipids and lipoproteins: a meta-analysis. Am J Clin Nutr, 1992. 56(2): p. 320-8.

34. Mantyselka, P., et al., Weight change and lipoprotein particle concentration and particle size: a cohort study with 6.5-year follow-up. Atherosclerosis, 2012. 223(1): p. 239-43.

35. Li, X.M., et al., Paradoxical association of enhanced cholesterol efflux with increased incident cardiovascular risks. Arterioscler Thromb Vasc Biol, 2013. 33(7): p. 1696-705. 
36. Voloshyna, I. and S. Seshadri, Infliximab reverses suppression of cholesterol efflux proteins by TNF-alpha: a possible mechanism for modulation of atherogenesis. 2014. 2014: p. 312647.

37. Selvin, E., N.P. Paynter, and T.P. Erlinger, The effect of weight loss on C-reactive protein: a systematic review. Arch Intern Med, 2007. 167(1): p. 31-9.

38. Brites, F., et al., Enhanced cholesterol efflux promotion in well-trained soccer players. Metabolism, 2004. 53(10): p. 1262-7.

39. Saleheen, D., et al., Association of HDL cholesterol efflux capacity with incident coronary heart disease events: a prospective case-control study. Lancet Diabetes Endocrinol, 2015. 3(7): p. 507-13.

40. Marsche, G., et al., Adiponectin predicts HDL cholesterol efflux capacity in adults irrespective of body mass index and fat distribution. J Clin Endocrinol Metab, 2017.

41. Asztalos, B.F., et al., Effects of weight loss, induced by gastric bypass surgery, on HDL remodeling in obese women. J Lipid Res, 2010. 51(8): p. 2405-12.

42. Arai, T., et al., Increased plasma cholesteryl ester transfer protein in obese subjects. A possible mechanism for the reduction of serum HDL cholesterol levels in obesity. Arterioscler Thromb, 1994. 14(7): p. 1129-36.

43. Purnell, J.Q., et al., Effect of weight loss with reduction of intra-abdominal fat on lipid metabolism in older men. J Clin Endocrinol Metab, 2000. 85(3): p. 977-82.

44. Tzotzas, T., et al., Early decreases in plasma lipid transfer proteins during weight reduction. Obesity (Silver Spring), 2006. 14(6): p. 1038-45.

45. Hayashibe, H., et al., Increased plasma cholesteryl ester transfer activity in obese children. Atherosclerosis, 1997. 129(1): p. 53-8.

46. Hirschler, V., et al., Impact of unhealthy lifestyle behaviors and obesity on cholesteryl ester transfer protein among adolescent males. Arch Med Res, 2011. 42(1): p. 53-9.

47. Jiang, X.C., et al., Mammalian adipose tissue and muscle are major sources of lipid transfer protein $m R N A$. J Biol Chem, 1991. 266(7): p. 4631-9.

48. Radeau, T., et al., Relationship of adipose tissue cholesteryl ester transfer protein (CETP) mRNA to plasma concentrations of CETP in man. Atherosclerosis, 1998. 139(2): p. 36976.

49. Dusserre, E., P. Moulin, and H. Vidal, Differences in mRNA expression of the proteins secreted by the adipocytes in human subcutaneous and visceral adipose tissues. Biochim Biophys Acta, 2000. 1500(1): p. 88-96.

50. Blauw, L.L., et al., Serum CETP concentration is not associated with measures of body fat: The NEO study. Atherosclerosis, 2016. 246: p. 267-73.

51. Guyard-Dangremont, V., et al., Competitive enzyme-linked immunosorbent assay of the human cholesteryl ester transfer protein (CETP). Clin Chim Acta, 1994. 231(2): p. 147-60.

52. Villard, E.F., et al., Elevated CETP activity improves plasma cholesterol efflux capacity from human macrophages in women. Arterioscler Thromb Vasc Biol, 2012. 32(10): p. 2341-9.

53. Scharnagl, H., et al., Decreased cholesterol efflux capacity in patients with low cholesteryl ester transfer protein plasma levels. Eur J Clin Invest, 2014. 44(4): p. 395-401. 


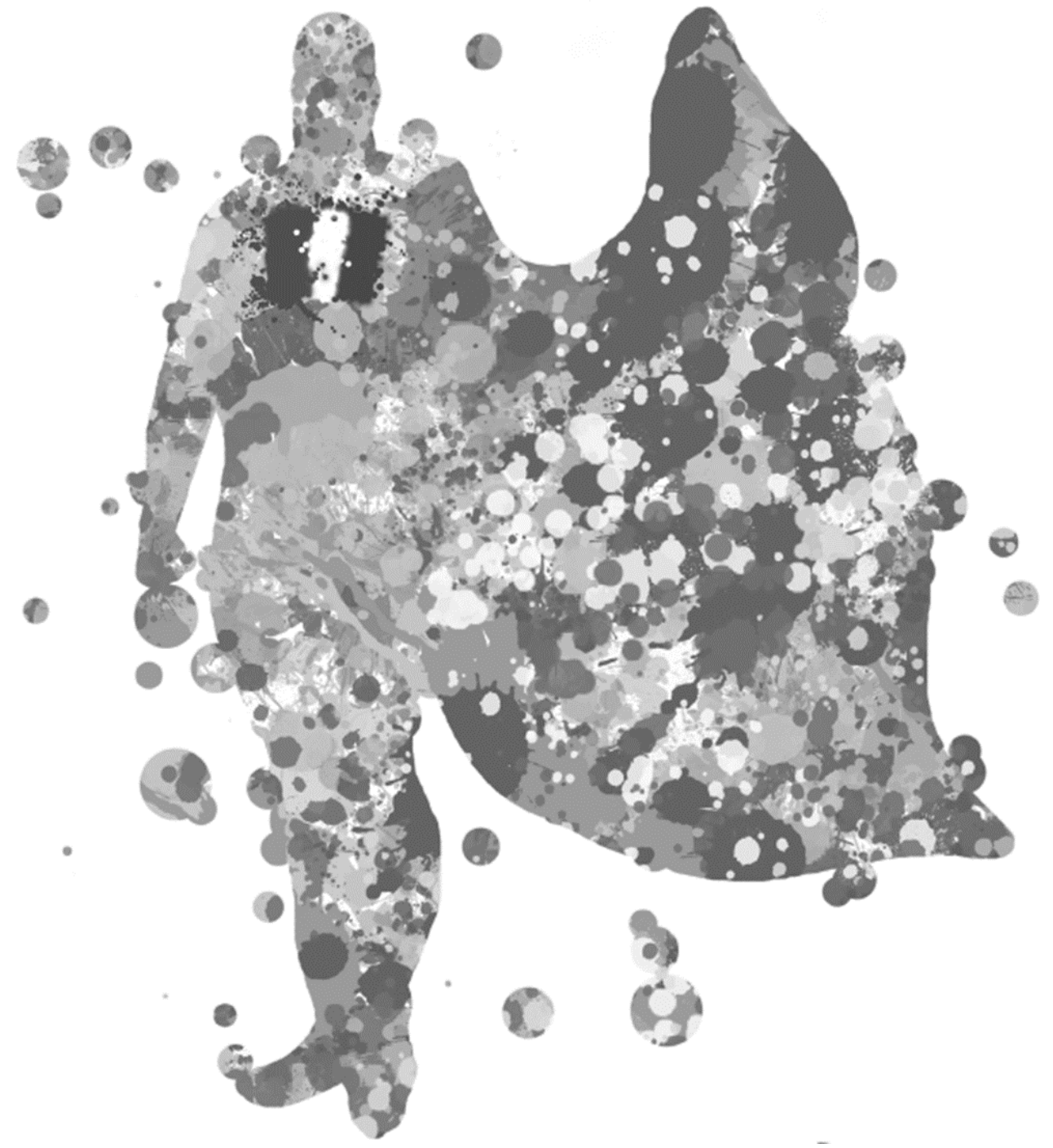




\section{Chapter 7}

General discussion 


\section{High-density lipoproteins and CVD risk}

Because cardiovascular diseases (CVD) are still the leading cause of morbidity and mortality worldwide [1], it becomes urgent to develop new therapeutic targets to improve cardiovascular health. A causal relationship between high levels of lowdensity lipoprotein cholesterol (LDL-C) and CVD has been established [2]. Moreover, lowering LDL-C concentrations by the use of statins has proven benefits in the prevention of CVD events [3]. However, despite the inverse relationship between high-density lipoprotein cholesterol (HDL-C) concentrations and CVD [4, 5], elevating HDL-C concentrations failed to reduce CVD risk [6]. This lack of benefit of increasing HDL-C concentrations might be due to the complexity of HDL particles, which vary in size and composition. Indeed, HDL metabolism and functionality can greatly differ between HDL subpopulations [7], and alterations in the protein composition of HDL have been associated with a variety of disorders, such as inflammation [8] and diabetes [9]. In this context, measuring only HDL-C concentrations may be a poor biomarker to assess CVD risk [10]. One of the main features of HDL particles is their ability to promote cholesterol efflux to remove the excess of accumulated cholesterol in from macrophages within the vessel wall, which is termed as reverse cholesterol transport (RCT) pathway. A strong inverse relationship between HDL-mediated cholesterol efflux capacity, and the prevalence and the incidence of coronary artery disease has been demonstrated in several epidemiological studies [11-13], and HDL-mediated cholesterol efflux was found to be a strong predictor of coronary artery disease (CAD) [14]. Therefore, the development of therapeutic interventions focusing on HDL-efflux capacity rather than on HDL-C concentrations is essential to tackle the CVD burden.

\section{Methods to measure HDL cholesterol efflux capacity}

There are three potential strategies to increase macrophage RCT: (1) improving cellular cholesterol efflux from macrophages; (2) enhancing HDL acceptor number and functionality; and (3) increasing hepatic uptake and biliary/intestinal excretion [15]. The present thesis focused on the first strategy, i.e. the efflux of cholesterol from macrophages.

As summarized in the chapter 2 of this thesis, several in vitro assays have been developed to quantify the ability of HDL particles to accept labeled cholesterol from macrophages. Radioactive assay, using ${ }^{3} \mathrm{H}$-cholesterol, is the most commonly used and is considered as the gold standard [16]. However, new assays using stable isotopes [17] or fluorescent tracers, such as BODIPY-cholesterol [18], have been 
developed, and are good alternatives to avoid the use of radioactive compounds. Recent studies have shown that results of the in vitro efflux assay using murine J774 macrophages, that naturally express the efflux transporters, labeled with ${ }^{3} \mathrm{H}$ cholesterol used in chapters 3 and 5 is negatively associated with CVD risk [11, 13]. In contrast, the BODIPY-cholesterol efflux assay was used in chapters $\mathbf{4}$ and $\mathbf{6}$. However, this fluorescence-labeled cholesterol efflux assay is also inversely associated with cardiovascular events, and a moderate correlation between both assays has been reported $(r=0.54 ; n=179)$ [12]. Regardless of the type of tracer used, the efflux-assay consists of three main steps: (1) donor cells are labeled with the cholesterol tracer; (2) labeled cells are incubated with the acceptor; (3) and the percentage of tracer effluxed from the cells and as such present in the supernatant is quantified [19]. The relative contribution of various efflux-mediated pathways, i.e. ABCA1-, ABCG1- and SR-BI-mediated efflux, has been quantified in human serum. It was found that cholesterol efflux from macrophages was predominantly due to the ABCA1-mediated pathway [20]. In addition, cholesterol efflux via the ABCA1 pathway is impaired in CAD patients [21], and ABCA1-knockout mice have increased atherosclerotic lesion formation [22]. Upregulating and measuring in vitro ABCA1-mediated cholesterol efflux could therefore provide additional information in assessing the risk of atherosclerosis [16]. However, these studies do not prove that impaired ABCA1-cholesterol efflux is causally related to atherosclerotic risk [23].

A limitation of these in vitro efflux assays is that - although they reflect the ability to mobilize free cholesterol from macrophages - they do not measure variation in other parts of the RCT pathway, such as the hydrolysis of cholesteryl esters, as well as the uptake into the liver and biliary excretion [15].

\section{Modulators of HDL cholesterol efflux capacity}

HDL is a very complex class of lipoproteins that varies in size, composition and functional properties. HDL metabolism and functionality may be related to many factors, such as genetic, molecular and environmental factors, i.e. weight loss and diet.

\section{Genetic determinants}

Associations between single nucleotide polymorphisms (SNPs) with HDL-C concentrations have been frequently investigated [24, 25]. The genetic variants associated with HDL-C concentrations include SNPs located in genes encoding for apolipoproteins, lipoprotein lipase, and transporters. However, the hypothesis that genetic polymorphisms linked to higher HDL-C concentrations are associated with 
a decrease in CVD risk has been challenged [26]. As HDL-mediated efflux capacity is a better target for CVD, it is of great interest to investigate genetic polymorphisms linked to this feature, rather than to HDL-C concentrations.

So far, only one study has investigated the impact of genetic polymorphisms on cholesterol efflux capacity. The main finding of this study was that SNPs located in genes involved in HDL metabolism, i.e. CETP, ABCA1, apoA-I, apolipoprotein A-II (apoA-II) and LIPC (lipoprotein lipase), were associated with plasma efflux capacity, independently of plasma HDL-C concentration and in a gender-specific manner. Interestingly, alleles of these identified SNPs associated with an increase in cholesterol efflux have been previously associated with a reduced risk of coronary heart disease [27]. In chapter 3 of this dissertation, we investigated the impact of selected SNPs coding for key genes involved in the RCT pathway. We found that SNPs located in ABCA1, ABCG1, LXR $\beta$ and CETP were significantly associated with cholesterol efflux capacity (Table 1). It has previously been reported that approximately $70 \%$ of inter-individual variation in HDL-C concentrations is explained by genetics polymorphisms [28]. However, the influence of genetic determinants on HDL cholesterol efflux appears to be rather low, accounting for only $\approx 6 \%$ [27].

A limitation of our study is the small number of subjects used. Indeed, analyses were performed in 107 subjects ( 50 men and 57 women), which might have been too small for any firm conclusions regarding the role of variation in genetic background. For comparison, the study of Villard and colleagues was performed in 450 subjects [27]. In addition, we did not investigate the relationship between the SNPs and CVD risk, and there is no evidence in the literature so far that the SNPs discussed in chapter 3 are related with a change in CVD risk. Therefore, more research is needed to establish whether these specific SNPs are related to an increased risk to develop CVD.

\section{Molecular factors: impact of microRNAs}

Vickers et al. have shown that HDL particles are carrying specific microRNAs (miRNAs) [29]. However, the exact functions of these specific HDL-miRNAs are not well known. In this context, we investigated if three of the most abundant HDLassociated miRNAs, namely miR-223, miR-92a and miR-135a, were associated with HDL-efflux capacity (chapter 4). Unfortunately, we did not find any association between the studied miRNAs with the ability of HDL to promote cholesterol efflux (Table 1). So far, only miR-223 has been reported to have an indirect effect on the promotion of cholesterol efflux [30]. In vitro, miR-223 can be exported from macrophages to HDL particles [29]. Subsequently, HDL particles deliver miR-223 to 
endothelial cells through SR-BI, suggesting a role of HDL-miRNAs in intercellular communication [31]. Upregulation of ABCA1 expression in J774 macrophages failed to increase miR-223 export to HDL [29, 32]. Because of their phospholipid core, HDLs can transport hydrophobic drugs through the circulation, addressing the potential of using HDL as a delivery vehicle for miRNA [33, 34].

Many miRNAs have been reported to be involved in lipoprotein metabolism and cholesterol homeostasis [35-37]. The best-characterized miRNAs linked to lipid and lipoprotein metabolism is miR-33. In humans, two isoforms of miR-33 are present: miR-33a and miR-33b, which are respectively located in SREBP2 and SREBP1 (sterol regulatory element-binding transcription protein). In contrast, only miR-33a exits in mice [38]. In vivo experiments have shown that miR-33a reduces HDL biogenesis in the liver, as well as cholesterol efflux from macrophages by inhibiting the expression of the ABCA1 transporter [39]. In mice, inhibition of miR-33 was associated with an increase in HDL-C concentrations and cholesterol efflux capacity. In addition, ABCA1 expression was upregulated in foam cells, resulting in regression of atherosclerosis [40]. Similarly, miR-26 was found to regulate in vitro cholesterol efflux from macrophages. Overexpression of miR-26 was associated with a reduction in cholesterol efflux, explained by the downregulation of ABCA1 expression in human and mouse macrophages targeting miR-33, as well as miR-26, may therefore be attractive targets for the prevention and treatment of CVD. However, no evidence has been reported so far that miR-33a/b and miR-26 are carried by HDL particles [41]. Therefore, more studies are needed to determine the complete HDL-miRNAs profile.

The majority of the population spends a large part of the day in the postprandial state and disturbances in postprandial lipoprotein metabolism are important risk markers for CVD [42, 43]. It is thus of clinical importance to consider postprandial measurements when addressing CVD risk. In chapter 4, we investigated for the first time the postprandial changes of miR-92a, miR-223 and miR-135a levels. A significant increase in both miR-92a and miR-223 levels following meal intake was found, while a trend was observed for miR-135a. However, the reasons for these changes are not clear. It is not yet known if the postprandial increases in miRNAs levels are due to the absorption of the miRNAs from food products, to an increase in the expression of genes coding for these specific miRNAs, or finally to changes in nutrients composition or variation in hormonal status. 


\section{Environmental factors: impact of weight loss and diet}

It has previously been reported that lifestyle can modulate HDL-efflux capacity, as reviewed in the chapter 2 of this thesis. In addition, the use of statins is a predictor of plasma cholesterol efflux capacity (chapter 3 ). As not much is known about the impact of diet on HDL-mediated cholesterol efflux, the potential beneficial effects of three food components on HDL-functionality were investigated in chapters 4 and 5 . Dark chocolate consumption is associated with a reduction in the risk to develop CVD [44]. These relations may be mediated through effects on HDL metabolism, although the impact of chocolate consumption on HDL-C concentrations is contradictory: one meta-analysis reported that flavonoid-rich cocoa consumption increased HDL-C concentrations [45], while 2 others found no effect of cocoa products on HDL-C concentrations [46, 47]. This raises the question if the beneficial effects of chocolate consumption on CVD risk may be attributed to an improvement in HDL-functionality. Therefore, the association between theobromine, a methylxanthine mainly found in dark chocolate [48], on HDL cholesterol efflux capacity has been investigated for the first time (chapter 4). Furthermore, plant sterol and plant stanol esters have been proven to be effective and safe functional food ingredients to reduce LDL-C concentrations [49]. They may also affect HDL-C concentrations. However, the effect of stanol consumption appears to be dependent on the HDL-C concentrations at baseline [50]. The aim of chapter $\mathbf{5}$ was to investigate the impact of plant sterol and stanol consumption on HDL functionality. In addition, as obesity is associated with a reduced ability of HDL to stimulate cholesterol efflux, we aimed to investigate if a decrease in BMI was associated with an improvement in cholesterol efflux capacity (chapter 6). The section below discusses the effects of these 3 dietary interventions on the ability of HDL to stimulate cholesterol efflux.

\section{Theobromine supplementation}

It is of interest to know which components in cocoa are responsible for the possible beneficial effect on CVD risk. We hypothesized that theobromine might be one of the active compounds. In one study, 4 weeks of cocoa powder consumption resulted in a small increase in apoA-I concentrations, while the increase was larger when consuming $850 \mathrm{mg}$ of pure theobromine for 4 weeks. In addition, only theobromine consumption increased HDL-C concentrations, whereas no effect was found in the cocoa group [51]. Until now, the effect of cocoa and/or theobromine consumption on HDL cholesterol efflux capacity has never been studied. In chapter 4 of this dissertation, we investigated for the first time the effect of theobromine consumption on HDL-efflux capacity. For this, 44 overweight and obese men and women with low baseline HDL-C concentrations consumed $500 \mathrm{mg}$ of theobromine for 4 weeks. 
Although we observed an increase in HDL-C concentrations, daily intake of theobromine did not change ABCA1-mediated cholesterol efflux. Similarly, theobromine did not change cholesterol efflux postprandially (Table 1). Our study therefore suggests that it is unlikely that the beneficial effect of dark chocolate consumption on CVD risk can be attributed to theobromine, and that other components from cocoa could have more beneficial properties. For instance, the flavonoids present in cocoa might be potential candidates [52], as they improve cholesterol efflux in vitro [53]. However, human interventions are less consistent as some studies found improvement in HDL-efflux capacity after anthocyanin supplementation [54, 55], while others found no effects of isoflavone supplementation [56,57]. In addition, combining the intake of theobromine with other bioactive compounds from cocoa might have synergistic effects, and therefore be more beneficial than when consumed alone. It was recently shown that consumption of a mixture of theobromine and caffeine together with flavanols increased the bioavailability of the flavanols, resulting in a more beneficial effect on vascular function, than when consuming flavanols alone [58]. However, if the same is true for cholesterol efflux remains to be determined.

\section{Plant sterol and stanol consumption}

To investigate the effect of plant sterol and plant stanol esters consumption on HDL functionality, data from 2 earlier studies with healthy subjects were used, including 66 men and 41 women. Study 1 consisted of a short-term intervention of 8 weeks, whereas study 2 was a long-term intervention of 85 weeks (chapter 5). As plant sterols and plant stanols are transported by lipoproteins, this raises the question whether the functionality of HDL particles can be influenced by their content in plant sterols and stanols. In both studies, plant stanol consumption decreased CETP mass, while no effect was found on CETP activity and HDL-mediated cholesterol efflux. In addition, plant sterol intake did not affect any of the tested parameters (Table 1). The fact that subjects did not have low HDL-C and high triacylglycerol (TAG) concentrations might have influenced the results. Indeed, plant stanol ester consumption was found to increase HDL-C concentrations in subjects with low HDL-C baseline concentrations. Similarly, high TAG baseline concentrations resulted in a more pronounced decrease in TAG concentrations [50]. Further studies in populations with low HDL-C and elevated TAG concentrations are therefore warranted to address the potential beneficial effects of plant sterol and stanol consumption on HDL metabolism and functionality. 


\section{Weight-loss intervention}

The impact of weight-loss induced by caloric restriction on cholesterol efflux was investigated in 52 abdominally obese men (chapter 6). At the end of the 6 weeks interventions, followed by 2 weeks of a weight-stable period, subjects had lost $10 \mathrm{~kg}$. We found that HDL particles from obese subjects have a lower ability to stimulate cholesterol efflux from macrophages than normal weight subjects, which agrees with earlier studies [59-61]. This might be related to the chronic low-grade inflammation present in obese subjects [62], which is associated with a reduction in efflux capacity [63]. This is supported by our findings, as we reported that CRP levels were significantly higher in the abdominally obese men, and negatively associated with cholesterol efflux (chapter 6).

Unfortunately, diet-induced weight loss failed to improve cholesterol efflux capacity (Table 1). This lack of effect might be explained by the duration of our study. Indeed, both HDL-C and apoA-I concentrations were increased only during the weight stable period, while a reduction was observed during the active weight-loss period $[64,65]$. This suggests that the weight-stable period of 2 weeks that we have used might have been too short to observe already an effect on HDL-efflux capacity. In addition, the relationship between fat distribution and cholesterol efflux has been investigated (chapter 6). Negative associations between efflux capacity with both visceral and subcutaneous fat were observed. However, after stepwise linear regression analysis, only BMI remained a predictor of cholesterol efflux, suggesting that the various fat depots are not differently related to cholesterol efflux. It should be noted however that the subjects in our study had a waist circumference between $102-110 \mathrm{~cm}$. Possibly, the range in the waist-to-hip ratio, which is an indicator of fat distribution throughout the body (i.e. abdominal versus visceral fat) [66], was not large enough to observe associations. 


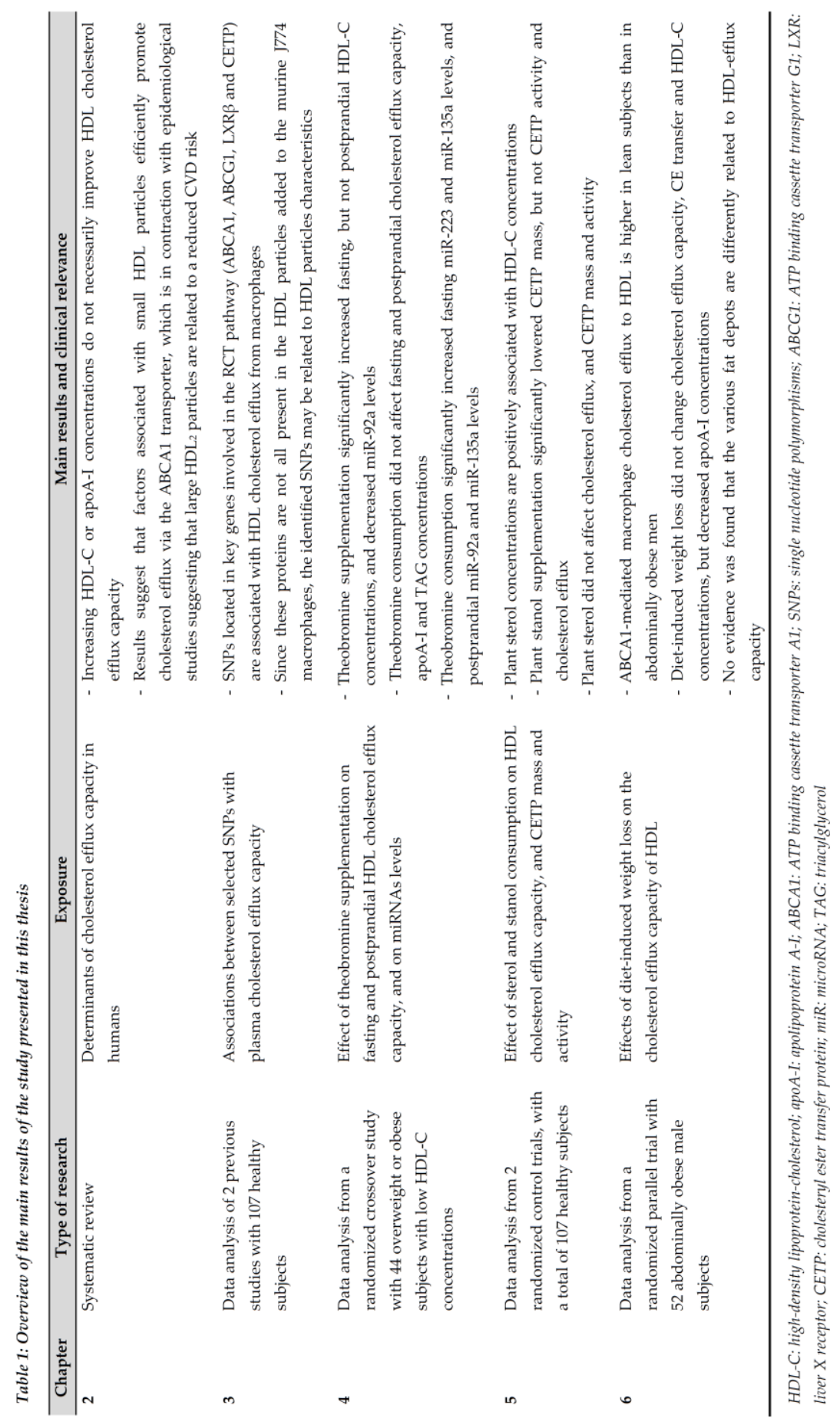




\section{Complexity of HDL experiments}

\section{Storage of HDL}

An important factor to take into account when working with HDL particles is samples storage. Indeed, biological samples are generally stored under freezing conditions prior to analysis. However, storage conditions including freezing and thawing might cause irreversible structural and functional damages of HDL. The use of freshly prepared HDL would be preferred, but this would lead to significant inter-experimental variability, as well as additional costs [67]. It was previously demonstrated that HDL-C concentrations are decreased by approximately $1 \%$ per month, after a 6 months of storage at $-20^{\circ} \mathrm{C}$ [68]. It is thus essential to know the impact of storage and freezing/thawing on the integrity of HDL preparations, as well as on HDL functional properties. The impact of short-term freezing at $-20^{\circ} \mathrm{C}$ had no effect on HDL particles size, as well as on the ability of HDL to promote cholesterol efflux, especially when sucrose was added to the samples. However, in that study the samples were only stored for up to 7 days, and damage may therefore have occurred after longer-term storage [67]. A recent study has investigated the impact of short- and long-term storage and freezing on the structural and functional properties of isolated HDL [69]. Structural damages leading to the loss of proteins from HDL were already observed after 2 weeks of storage at $-20^{\circ} \mathrm{C}$ or $-70^{\circ} \mathrm{C}$, and HDL remained stable at $4^{\circ} \mathrm{C}$ for up to 2 weeks only. In addition, flash-freezing HDL samples with nitrogen before storage did not prevent these damages. The use of cryoprotectants before storage, such as sucrose and glycerol, was able to protect HDL from storage damages. Cholesterol efflux capacity was significantly decreased after 5 weeks of storage at $4^{\circ} \mathrm{C}$. Surprisingly, when stored at $-20^{\circ} \mathrm{C}$ or $-70^{\circ} \mathrm{C}$, cholesterol efflux was increased, from 5 weeks onwards. The use of cryoprotectants did not affect cholesterol efflux measurements, suggesting that there is not need to remove them before performing the efflux measurements. The increase in cholesterol efflux observed can be explained by the loss of proteins due to the freezing process. Indeed, this leads to the formation of lipid-poor apoA-I, that might have structural and functional similarities with pre $\beta-1 \mathrm{HDL}$, the primary acceptor of cholesterol from ABCA1 [69]. A limitation of that study is that it was performed on HDL samples isolated by ultracentrifugation. Therefore, the effects of storage on HDL samples isolated with other methods, such as FPLC, immuno-affinity chromatography or apoB precipitations, might be different and warrant further investigations. Nevertheless, HDL being very complex particles, it is necessary to pay careful attention to HDL samples storage, as it might profoundly affect structural and functional properties. In addition, storing HDL samples together with cryoprotectants may be useful in order to preserve the integrity and the functionality of HDL. 


\section{Stability of HDL-miRNAs}

As storage temperature and time can alter HDL samples, it is important to know if the stability of HDL-miRNAs is also affected by storage conditions. So far, the stability of only 2 miRNAs associated with HDL has been investigated, i.e. miR-135a and miR-223. Both HDL-miR-135a and HDL-miR-223 were stable at different temperatures (room temperature; $4^{\circ} \mathrm{C} ;-20^{\circ} \mathrm{C}$ and $-80^{\circ} \mathrm{C}$ ), as well as up to five freezethaw cycles [70]. Based on these data, it can be assumed that the miRNAs analyzed in chapter 4 remained stable throughout the study. However, HDL carries many others miRNAs, such as miR-92a (chapter 4), and some of these might be more sensitive to storage conditions than others. Whether these results can be extrapolated to the other HDL-miRNAs warrants therefore additional studies. It was also reported that the stability of miRNAs in plasma is dependent on their number of AU or UA motifs in their sequences: the higher, the less stable the miRNA is [71]; but whether this is also true for HDL-miRNAs remains to be determined.

\section{Isolation of HDL particles for miRNAs analysis}

In addition to HDL, miRNAs are carried by extracellular vesicles, such as exosomes $[29,72]$. Exosomes are involved in various biological processes, including angiogenesis, intercellular communication, cell survival, inflammation and immune response, coagulation, and waste management [72]. As reported in the miRandola database and depicted in Figure 1, miRNAs are more abundantly present in exosomes than in HDL [41]. 


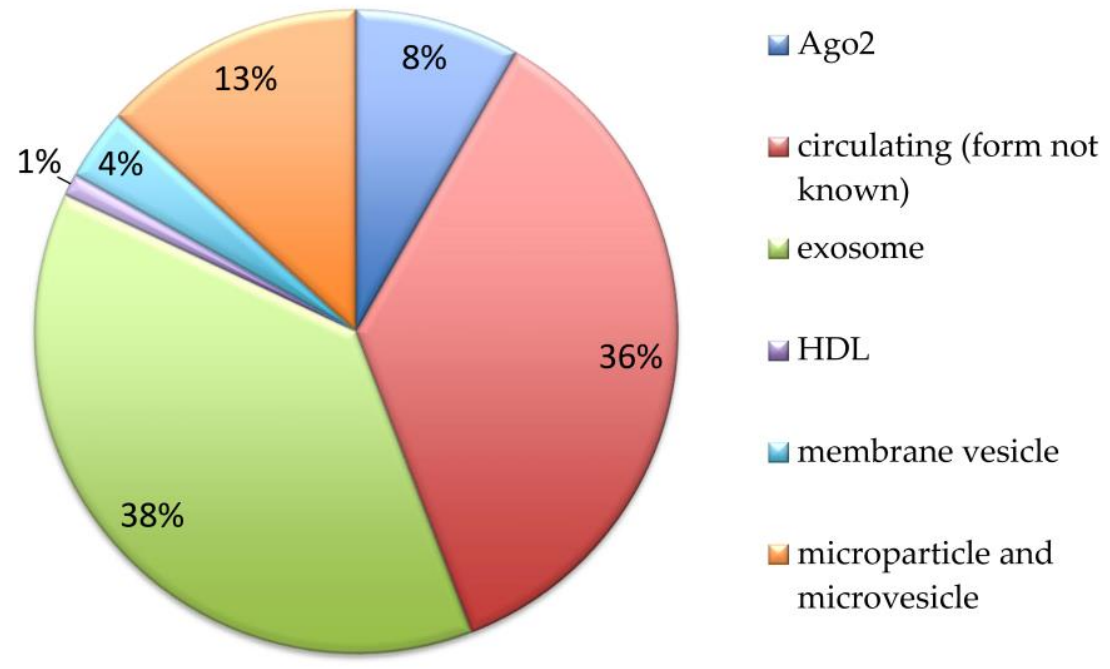

Figure 6: Proportions of extracellular forms of miRNAs as reported in the literature [41].

Ago2 (Argonaute2) complexes are RNA-binding proteins involved in RNA silencing [73]. The term "circulating" indicates that the authors did not mention the form in which the miRNA is present.

Furthermore, one of the characteristics of exosomes is that they have a similar density as HDL particles [29, 74] (Table 2). In this context, isolating HDL particles using only density gradient ultracentrifugation would result in a contamination of HDL particles with exosomes [74]. Thus, the contribution of HDL as carriers of miRNAs will be overestimated, as exosomes also contribute to the total miRNAs pool, and some miRNAs are found in both exosomes and HDL particles, such as miR-223 [75]. Because HDL and exosomes have different sizes (Table 2), Mitchell et al. have recommended a protocol using tandem HDL isolation with density-gradient ultracentrifugation followed by fast-protein-liquid chromatography (FPLC) to isolate highly purified HDL [76]. 
Table 2: Size and density of lipoproteins and exosomes [76].

\begin{tabular}{|c|c|c|c|c|c|c|c|c|c|c|c|c|c|c|}
\hline \multirow{5}{*}{ 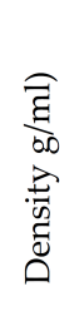 } & $0.95-1.006$ & & & & & & \multicolumn{5}{|c|}{ VLDL } & \multicolumn{3}{|c|}{ Chylomicrons } \\
\hline & $1.006-1.02$ & & & & \multicolumn{2}{|c|}{ IDL } & & & & & & & & \\
\hline & $1.02-1.06$ & & & LDL & & & & & & & & & & \\
\hline & $1.06-1.12$ & \multirow{2}{*}{\multicolumn{2}{|c|}{ HDL }} & & & & \multirow{2}{*}{\multicolumn{8}{|c|}{ Exosomes }} \\
\hline & $1.12-1.21$ & & & & & & & & & & & & & \\
\hline & & 0 & 10 & 20 & 30 & 40 & 50 & 60 & 70 & 80 & 90 & 100 & 110 & 120 \\
\hline & & \multicolumn{13}{|c|}{ IIIII) } \\
\hline
\end{tabular}

However, this method is time consuming and cumbersome. In our hands, isolation of HDL particles using only FPLC resulted in the detection of 4 exosomal markers, i.e. CD63, CD81, CD9 and HsP70, suggesting the presence of exosomes in the HDL samples (Figure 2 - panel 1).

Another and faster approach to isolate HDL is by performing immuno-affinity chromatography, using goat anti-human apoA-I antibodies conjugated to Sepahrose-4B beads $[29,77]$. When using this technique, HDLs specifically bind to the apoA-I beads. The bound HDLs are subsequently eluted and the recovered samples are finally concentrated. An advantage of this method is that the apoA-I beads can be stored for a long period at $4^{\circ} \mathrm{C}$ and re-used multiple times. In addition, purification of samples using immuno-affinity separation of proteins has proven to be an effective approach [78]. When using this technic, CD63 was not detected in the recovered supernatant. However, results for the other exosomal markers were less clear (Figure 2 - panel 2).

In chapter 4, HDL was isolated by precipitation of apoB-containing lipoproteins, and exosomes were therefore still present in the supernatant used for the miRNAs analysis. Indeed, when using a mixture of phosphotungstic acid and magnesium chloride (Phosphotungstate Precipitant, Roche Diagnostics, Germany) to precipitate apoB-containing lipoproteins, all the exosomal markers but Hsp70 were detected by western blot (Figure 2 - panel 3). Similarly to HDL, exosomal miRNAs can be delivered to other cells and thus influence gene expression, making them a key player in cell-to-cell communication [79]. 


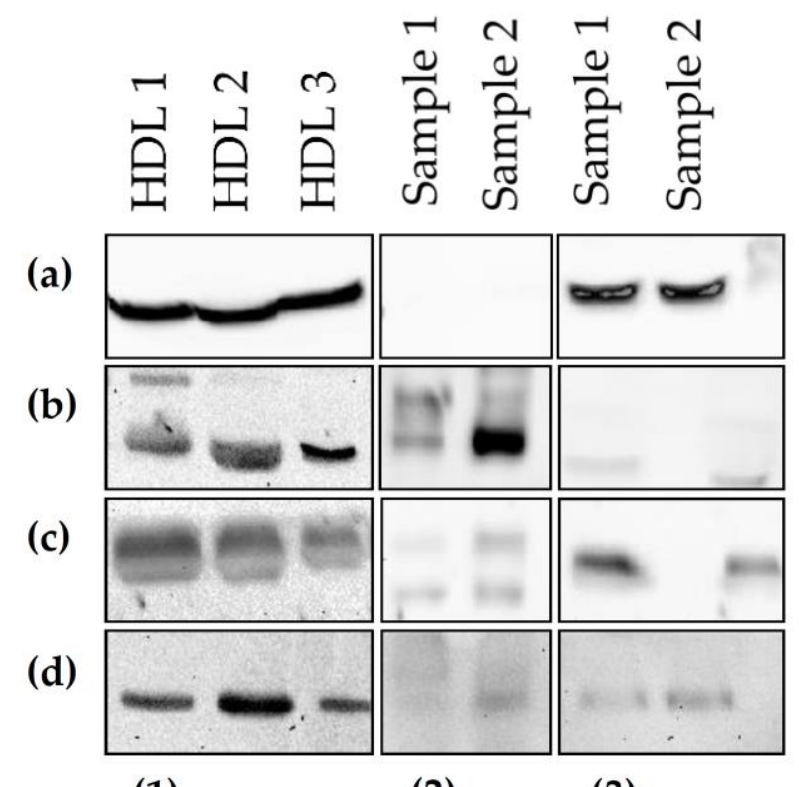

(1)

(2)

(3)

Figure 7: Detection of exosomal markers by western blot in HDL samples isolated by FPLC (1), immunoaffinity chromatography using apoA1 beads (2), and precipitation of apoB-containing lipoproteins (3).

(a) CD63: 53kDa; (b) Hsp70:53-70kDa; (c) CD9: 28kDa; (d) CD81: 26kDa;

HDL 1 = pooled FPLC fractions 16 to 18; HDL 2 = pooled FPLC fractions 19 to 21; HDL 3 = pooled FPLC fractions 22 to 24 .

Samples for panels (2) and (3) were run in duplicate.

In the circulation, miRNAs can also be associated with Ago2, which is involved in the silencing of mRNA [80, 81]. Both miR-92a [80] and miR-223 [82] are carried by Ago2. However, if Ago2-miRNAs can be released from cells and be taken up by recipient cells is not known yet. In addition, if Ago2 is co-isolated with HDL particles when using any of the isolation technics discussed above has never been investigated, and therefore deserves more attention.

To summarize, the isolation of highly pure HDL particles for miRNAs analysis is challenging. Depending on the method used, HDL is likely to be co-isolated together with other forms of miRNAs-carriers. Therefore, more research is still needed to establish the optimal and simplest method for HDL isolation and purification. 


\section{Conclusion and future directions}

The studies described in this thesis focused on factors that may modulate and improve the ability of HDL particles to stimulate cholesterol-efflux capacity. We have first demonstrated that cholesterol efflux is related to SNPs of genes involved in HDL metabolism. It is of interest to examine if these relations persist when a larger number of subjects are included. However, our identified SNPs have not been associated so far with CAD by genome-wide association studies (GWAS) [83, 84]. Therefore, studies should be initiated to examine if these SNPs are related to CVD risk (chapter 3 ). We then investigated the effect of several dietary compounds on HDL-related parameters. Theobromine consumption for a period of 4 weeks did not increase HDL-efflux capacity. However, theobromine did have an effect on the levels of the miR-92a (chapter 4), which is involved in angiogenesis and atherosclerosis [85-87]. This study also showed that HDL-mediated cholesterol efflux capacity as well as miR-223 and miR-92a levels were significantly increased after meal intake, as compared to the fasting situation (chapter 4). Plant stanol consumption decreased CETP mass, while no effect was found on CETP activity and cholesterol efflux. In addition, plant sterol consumption did not affect any of these parameters. This lack of effects might be attributed to our study population. Additional intervention studies performed in subjects with a dyslipidemic profile, i.e. low HDL-C and high TAG concentrations, would be of interest to better understand the effects of plant sterol and plant stanol esters on HDL metabolism and functionality (chapter 5). Finally, we investigated if weight reduction was associated with an improvement in cholesterol efflux, but no beneficial effect was found. However, at baseline, BMI was found to be an important predictor cholesterol efflux (chapter 6).

HDLs are very complex particles, varying in size and composition, and different HDL subfractions differently affect cholesterol efflux capacity. Further studies should therefore focus on the effect of interventions on each HDL subfraction. In addition, several transporters modulate cholesterol efflux. In our studies, the cholesterol efflux assays used focused on ABCA1-mediated cholesterol efflux from macrophages. However, other pathways, such as ABCG1 and SR-BI might be of interest as well, as they measure the efflux of cholesterol from macrophages to other HDL particles, i.e. HDL2 and HDL3. However, ABCG1- and SR-BI-mediated efflux pathways have not been related to CVD risk so far. Therefore, future research should address the involvement of these others transporters. The individual efficiencies of each transporter could be assessed by using BHK (baby hamster kidney) or CHOK1 (Chinese Hamster Ovary-K1) cells, which do normally not express these transporters, and can therefore be transfected with either ABCA1, ABCG1 or SR-BI. Additionally, even though in vitro ABCA1-mediated efflux assays have been 
negatively associated with CVD risk, it is necessary to establish if these in vitro results are causally related to in vivo CVD risk.

Since the discovery of miRNAs in 1993, the interest and knowledge in miRNAs has grown exponentially [88]. Because the miRNA signature is changed in many diseases, they have the potential to be used as biomarkers for disease diagnosis, as well as possible preventive and therapeutic targets. It was only until recently that miRNAs were discovered to be transported by HDL particles. In addition to the naturally endogenous miRNAs transported, HDL can also be loaded with specific miRNAs and deliver these exogenous miRNAs to recipient cells expressing SR-BI [29], making the use of HDL as a miRNAs-delivery vehicle attractive. However, the selective uptake of HDL by tissues and the roles of these HDL-miRNAs in health and disease are not well understood. It is also not known, how many miRNAs are exclusively transported by HDL particles. Extracellular vesicles, such as exosomes, are also carrying miRNAs and are important regulators of numerous biological processes. Given the large amount of miRNAs present in exosomes, further studies should focus on analyzing the exosome-miRNAs content, as well as their effect on cholesterol efflux capacity, and ultimately on CVD risk. There are currently 40 clinical trials investigating the impact of miRNAs on CVD (ClinicalTrials.gov). However, more insight into the miRNAs content of each extracellular carrier, as well as the transporters involved in their export and delivery to recipient cells is needed to gain more knowledge on their potential effects on the development of CVD. 


\section{References}

1. Organization, W.H. Cardiovascular diseases (CVDs) fact sheet No. 317. May 2017; Available from: http://www.who.int/mediacentre/factsheets/fs317/en/.

2. Stein, E.A., Low-density lipoprotein cholesterol reduction and prevention of cardiovascular disease. Mayo Clin Proc, 2009. 84(4): p. 307-9.

3. Fulcher, J., et al., Efficacy and safety of LDL-lowering therapy among men and women: meta-analysis of individual data from 174,000 participants in 27 randomised trials. Lancet, 2015. 385(9976): p. 1397-405.

4. Barter, P., et al., High density lipoproteins (HDLs) and atherosclerosis; the unanswered questions. Atherosclerosis, 2003. 168(2): p. 195-211.

5. Choi, B.G., et al., The role of high-density lipoprotein cholesterol in the prevention and possible treatment of cardiovascular diseases. Curr Mol Med, 2006. 6(5): p. 571-87.

6. Rader, D.J. and G.K. Hovingh, HDL and cardiovascular disease. Lancet, 2014. 384(9943): p. 618-25.

7. Asztalos, B.F., M. Tani, and E.J. Schaefer, Metabolic and functional relevance of HDL subspecies. Curr Opin Lipidol, 2011. 22(3): p. 176-85.

8. Vaisar, T., et al., Inflammatory remodeling of the HDL proteome impairs cholesterol efflux capacity. J Lipid Res, 2015. 56(8): p. 1519-30.

9. Gordon, S.M., et al., The effects of type 2 diabetes on lipoprotein composition and arterial stiffness in male youth. Diabetes, 2013. 62(8): p. 2958-67.

10. Ronsein, G.E. and J.W. Heinecke, Time to ditch HDL-C as a measure of HDL function? Curr Opin Lipidol, 2017. 28(5): p. 414-418.

11. Khera, A.V., et al., Cholesterol efflux capacity, high-density lipoprotein function, and atherosclerosis. N Engl J Med, 2011. 364(2): p. 127-35.

12. Rohatgi, A., et al., HDL cholesterol efflux capacity and incident cardiovascular events. N Engl J Med, 2014. 371(25): p. 2383-93.

13. Saleheen, D., et al., Association of HDL cholesterol efflux capacity with incident coronary heart disease events: a prospective case-control study. Lancet Diabetes Endocrinol, 2015. 3(7): p. 507-13.

14. Ishikawa, T., et al., High-density lipoprotein cholesterol efflux capacity as a relevant predictor of atherosclerotic coronary disease. Atherosclerosis, 2015. 242(1): p. 318-22.

15. Khera, A.V. and D.J. Rader, Future therapeutic directions in reverse cholesterol transport. Curr Atheroscler Rep, 2010. 12(1): p. 73-81.

16. Hafiane, A. and J. Genest, High density lipoproteins: Measurement techniques and potential biomarkers of cardiovascular risk. BBA Clin, 2015. 3: p. 175-88.

17. Brown, R.J., et al., Cholesterol efflux analyses using stable isotopes and mass spectrometry. Anal Biochem, 2013. 433(1): p. 56-64.

18. Sankaranarayanan, S., et al., A sensitive assay for ABCA1-mediated cholesterol efflux using BODIPY-cholesterol. J Lipid Res, 2011. 52(12): p. 2332-40.

19. Litvinov, D.Y., et al., Cholesterol Efflux and Reverse Cholesterol Transport: Experimental Approaches. Curr Med Chem, 2016. 23(34): p. 3883-3908. 
20. de la Llera-Moya, M., et al., The ability to promote efflux via ABCA1 determines the capacity of serum specimens with similar high-density lipoprotein cholesterol to remove cholesterol from macrophages. Arterioscler Thromb Vasc Biol, 2010. 30(4): p. 796-801.

21. Shao, B., et al., Humans with atherosclerosis have impaired ABCA1 cholesterol efflux and enhanced high-density lipoprotein oxidation by myeloperoxidase. Circ Res, 2014. 114(11): p. 1733-42.

22. Rader, D.J., et al., The role of reverse cholesterol transport in animals and humans and relationship to atherosclerosis. J Lipid Res, 2009. 50 Suppl: p. S189-94.

23. Cuchel, M. and D.J. Rader, Macrophage reverse cholesterol transport: key to the regression of atherosclerosis? Circulation, 2006. 113(21): p. 2548-55.

24. Chen, S.N., et al., Candidate genetic analysis of plasma high-density lipoprotein-cholesterol and severity of coronary atherosclerosis. BMC Med Genet, 2009. 10: p. 111.

25. Peloso, G.M., et al., Common genetic variation in multiple metabolic pathways influences susceptibility to low HDL-cholesterol and coronary heart disease. J Lipid Res, 2010. 51(12): p. 3524-32.

26. Voight, B.F., et al., Plasma HDL cholesterol and risk of myocardial infarction: a mendelian randomisation study. Lancet, 2012. 380(9841): p. 572-80.

27. Villard, E.F., et al., Genetic determination of plasma cholesterol efflux capacity is genderspecific and independent of HDL-cholesterol levels. Arterioscler Thromb Vasc Biol, 2013. 33(4): p. 822-8.

28. Wang, X. and B. Paigen, Genetics of variation in HDL cholesterol in humans and mice. Circ Res, 2005. 96(1): p. 27-42.

29. Vickers, K.C., et al., MicroRNAs are transported in plasma and delivered to recipient cells by high-density lipoproteins. Nat Cell Biol, 2011. 13(4): p. 423-33.

30. Vickers, K.C., et al., MicroRNA-223 coordinates cholesterol homeostasis. Proc Natl Acad Sci U S A, 2014. 111(40): p. 14518-23.

31. Tabet, F., et al., HDL-transferred microRNA-223 regulates ICAM-1 expression in endothelial cells. Nat Commun, 2014. 5: p. 3292.

32. Michell, D.L. and K.C. Vickers, Lipoprotein carriers of microRNAs. Biochim Biophys Acta, 2016.

33. Michell, D.L. and K.C. Vickers, HDL and microRNA therapeutics in cardiovascular disease. Pharmacol Ther, 2016. 168: p. 43-52.

34. Mo, Z.C., et al., A high-density lipoprotein-mediated drug delivery system. Adv Drug Deliv Rev, 2016. 106(Pt A): p. 132-147.

35. Rayner, K.J. and K.J. Moore, MicroRNA control of high-density lipoprotein metabolism and function. Circ Res, 2014. 114(1): p. 183-92.

36. DiMarco, D.M. and M.L. Fernandez, The Regulation of Reverse Cholesterol Transport and Cellular Cholesterol Homeostasis by MicroRNAs. Biology (Basel), 2015. 4(3): p. 494511.

37. Desgagne, V., L. Bouchard, and R. Guerin, microRNAs in lipoprotein and lipid metabolism: from biological function to clinical application. Clin Chem Lab Med, 2017. 55(5): p. 667-686.

38. Najafi-Shoushtari, S.H., et al., MicroRNA-33 and the SREBP host genes cooperate to control cholesterol homeostasis. Science, 2010. 328(5985): p. 1566-9. 
39. Rayner, K.J., et al., MiR-33 contributes to the regulation of cholesterol homeostasis. Science, 2010. 328(5985): p. 1570-3.

40. Rayner, K.J., et al., Antagonism of miR-33 in mice promotes reverse cholesterol transport and regression of atherosclerosis. J Clin Invest, 2011. 121(7): p. 2921-31.

41. Russo, F., et al., miRandola 2017: a curated knowledge base of non-invasive biomarkers. Nucleic Acids Res, 2017.

42. Karpe, F., Postprandial lipoprotein metabolism and atherosclerosis. J Intern Med, 1999. 246(4): p. 341-55.

43. Cohn, J.S., Are we ready for a prospective study to investigate the role of chylomicrons in cardiovascular disease? Atheroscler Suppl, 2008. 9(2): p. 15-8.

44. Buitrago-Lopez, A., et al., Chocolate consumption and cardiometabolic disorders: systematic review and meta-analysis. Bmj, 2011. 343: p. d4488.

45. Shrime, M.G., et al., Flavonoid-rich cocoa consumption affects multiple cardiovascular risk factors in a meta-analysis of short-term studies. J Nutr, 2011. 141(11): p. 1982-8.

46. Jia, L., et al., Short-term effect of cocoa product consumption on lipid profile: a meta-analysis of randomized controlled trials. Am J Clin Nutr, 2010. 92(1): p. 218-25.

47. Tokede, O.A., J.M. Gaziano, and L. Djousse, Effects of cocoa products/dark chocolate on serum lipids: a meta-analysis. Eur J Clin Nutr, 2011. 65(8): p. 879-86.

48. Kelly, C.J., Effects of theobromine should be considered in future studies. Am J Clin Nutr, 2005. 82(2): p. 486-7; author reply 487-8.

49. Plat, J. and R.P. Mensink, Plant stanol and sterol esters in the control of blood cholesterol levels: mechanism and safety aspects. Am J Cardiol, 2005. 96(1A): p. 15D-22D.

50. Naumann, E., et al., The baseline serum lipoprotein profile is related to plant stanol induced changes in serum lipoprotein cholesterol and triacylglycerol concentrations. J Am Coll Nutr, 2008. 27(1): p. 117-26.

51. Neufingerl, N., et al., Effect of cocoa and theobromine consumption on serum HDLcholesterol concentrations: a randomized controlled trial. Am J Clin Nutr, 2013. 97(6): p. 1201-9.

52. Oracz, J., D. Zyzelewicz, and E. Nebesny, The content of polyphenolic compounds in cocoa beans (Theobroma cacao L.), depending on variety, growing region, and processing operations: a review. Crit Rev Food Sci Nutr, 2015. 55(9): p. 1176-92.

53. Millar, C.L., Q. Duclos, and C.N. Blesso, Effects of Dietary Flavonoids on Reverse Cholesterol Transport, HDL Metabolism, and HDL Function. 2017. 8(2): p. 226-239.

54. Qin, Y., et al., Anthocyanin supplementation improves serum LDL-and HDL-cholesterol concentrations associated with the inhibition of cholesteryl ester transfer protein in dyslipidemic subjects. Am J Clin Nutr, 2009. 90(3): p. 485-92.

55. Zhu, Y., et al., Anthocyanin supplementation improves HDL-associated paraoxonase 1 activity and enhances cholesterol efflux capacity in subjects with hypercholesterolemia. J Clin Endocrinol Metab, 2014. 99(2): p. 561-9.

56. Tormala, R.M., et al., Serum cholesterol efflux potential in postmenopausal women treated with isolated isoflavones. Menopause, 2006. 13(1): p. 96-101.

57. Badeau, R., et al., Effect of isolated isoflavone supplementation on ABCA1-dependent cholesterol efflux potential in postmenopausal women. Menopause, 2007. 14(2): p. 293-9.

58. Sansone, R., et al., Methylxanthines enhance the effects of cocoa flavanols on cardiovascular function: randomized, double-masked controlled studies. 2017. 105(2): p. 352-360. 
59. Sasahara, T., et al., Cholesterol transport between cells and high density lipoprotein subfractions from obese and lean subjects. J Lipid Res, 1998. 39(3): p. 544-54.

60. Autran, D., et al., Basal and postprandial serum-promoted cholesterol efflux in normolipidemic subjects: Importance of fat mass distribution. Metabolism, 2001. 50(11): p. 1330-5.

61. Attia, N., et al., Impact of android overweight or obesity and insulin resistance on basal and postprandial SR-BI and ABCA1-mediated serum cholesterol efflux capacities. Atherosclerosis, 2010. 209(2): p. 422-9.

62. Wang, Z. and T. Nakayama, Inflammation, a link between obesity and cardiovascular disease. Mediators Inflamm, 2010. 2010: p. 535918.

63. Voloshyna, I. and S. Seshadri, Infliximab reverses suppression of cholesterol efflux proteins by TNF-alpha: a possible mechanism for modulation of atherogenesis. 2014. 2014: p. 312647.

64. Dattilo, A.M. and P.M. Kris-Etherton, Effects of weight reduction on blood lipids and lipoproteins: a meta-analysis. Am J Clin Nutr, 1992. 56(2): p. 320-8.

65. Asztalos, B.F., et al., Effects of weight loss, induced by gastric bypass surgery, on HDL remodeling in obese women. J Lipid Res, 2010. 51(8): p. 2405-12.

66. Egger, G., The case for using waist to hip ratio measurements in routine medical checks. Med J Aust, 1992. 156(4): p. 280-5.

67. Kekulawala, J.R., et al., Impact of freezing on high-density lipoprotein functionality. Anal Biochem, 2008. 379(2): p. 213-5.

68. Ekbom, T., et al., Decrease in high density lipoprotein cholesterol during prolonged storage. CELL Study Group. Scand J Clin Lab Invest, 1996. 56(2): p. 97-101.

69. Holzer, M., et al., HDL structure and function is profoundly affected when stored frozen in the absence of cryoprotectants. J Lipid Res, 2017.

70. Ishikawa, H., et al., Stability of serum high-density lipoprotein-microRNAs for preanalytical conditions. Ann Clin Biochem, 2017. 54(1): p. 134-142.

71. Balzano, F., et al., miRNA Stability in Frozen Plasma Samples. Molecules, 2015. 20(10): p. 19030-40.

72. Yuana, Y., A. Sturk, and R. Nieuwland, Extracellular vesicles in physiological and pathological conditions. Blood Rev, 2013. 27(1): p. 31-9.

73. Creemers, E.E., A.J. Tijsen, and Y.M. Pinto, Circulating microRNAs: novel biomarkers and extracellular communicators in cardiovascular disease? Circ Res, 2012. 110(3): p. 48395.

74. Yuana, Y., et al., Co-isolation of extracellular vesicles and high-density lipoproteins using density gradient ultracentrifugation. J Extracell Vesicles, 2014. 3.

75. Chen, Y., et al., Increased Circulating Exosomal miRNA-223 Is Associated with Acute Ischemic Stroke. Front Neurol, 2017. 8: p. 57.

76. Michell, D.L., et al., Isolation of High-density Lipoproteins for Non-coding Small RNA Quantification. J Vis Exp, 2016(117).

77. Tabet, F., et al., High-Density Lipoprotein-Associated miR-223 Is Altered after DietInduced Weight Loss in Overweight and Obese Males. PLoS One, 2016. 11(3): p. e0151061.

78. Huang, L., et al., Immunoaffinity separation of plasma proteins by Ig Y microbeads: meeting the needs of proteomic sample preparation and analysis. Proteomics, 2005. 5(13): p. 331428. 
79. Zhang, J., et al., Exosome and exosomal microRNA: trafficking, sorting, and function. Genomics Proteomics Bioinformatics, 2015. 13(1): p. 17-24.

80. Arroyo, J.D., et al., Argonaute2 complexes carry a population of circulating microRNAs independent of vesicles in human plasma. Proc Natl Acad Sci U S A, 2011. 108(12): p. 5003-8.

81. Turchinovich, A., et al., Characterization of extracellular circulating microRNA. Nucleic Acids Res, 2011. 39(16): p. 7223-33.

82. Li, L., et al., Argonaute 2 complexes selectively protect the circulating microRNAs in cellsecreted microvesicles. PLoS One, 2012. 7(10): p. e46957.

83. McPherson, R. and A. Tybjaerg-Hansen, Genetics of Coronary Artery Disease. Circ Res, 2016. 118(4): p. 564-78.

84. Webb, T.R., et al., Systematic Evaluation of Pleiotropy Identifies 6 Further Loci Associated With Coronary Artery Disease. J Am Coll Cardiol, 2017. 69(7): p. 823-836.

85. Bonauer, A., et al., MicroRNA-92a controls angiogenesis and functional recovery of ischemic tissues in mice. Science, 2009. 324(5935): p. 1710-3.

86. Loyer, X., et al., Inhibition of microRNA-92a prevents endothelial dysfunction and atherosclerosis in mice. Circ Res, 2014. 114(3): p. 434-43.

87. Niculescu, L.S., et al., MiR-486 and miR-92a Identified in Circulating HDL Discriminate between Stable and Vulnerable Coronary Artery Disease Patients. PLoS One, 2015. 10(10): p. e0140958.

88. Almeida, M.I., R.M. Reis, and G.A. Calin, MicroRNA history: discovery, recent applications, and next frontiers. Mutat Res, 2011. 717(1-2): p. 1-8. 


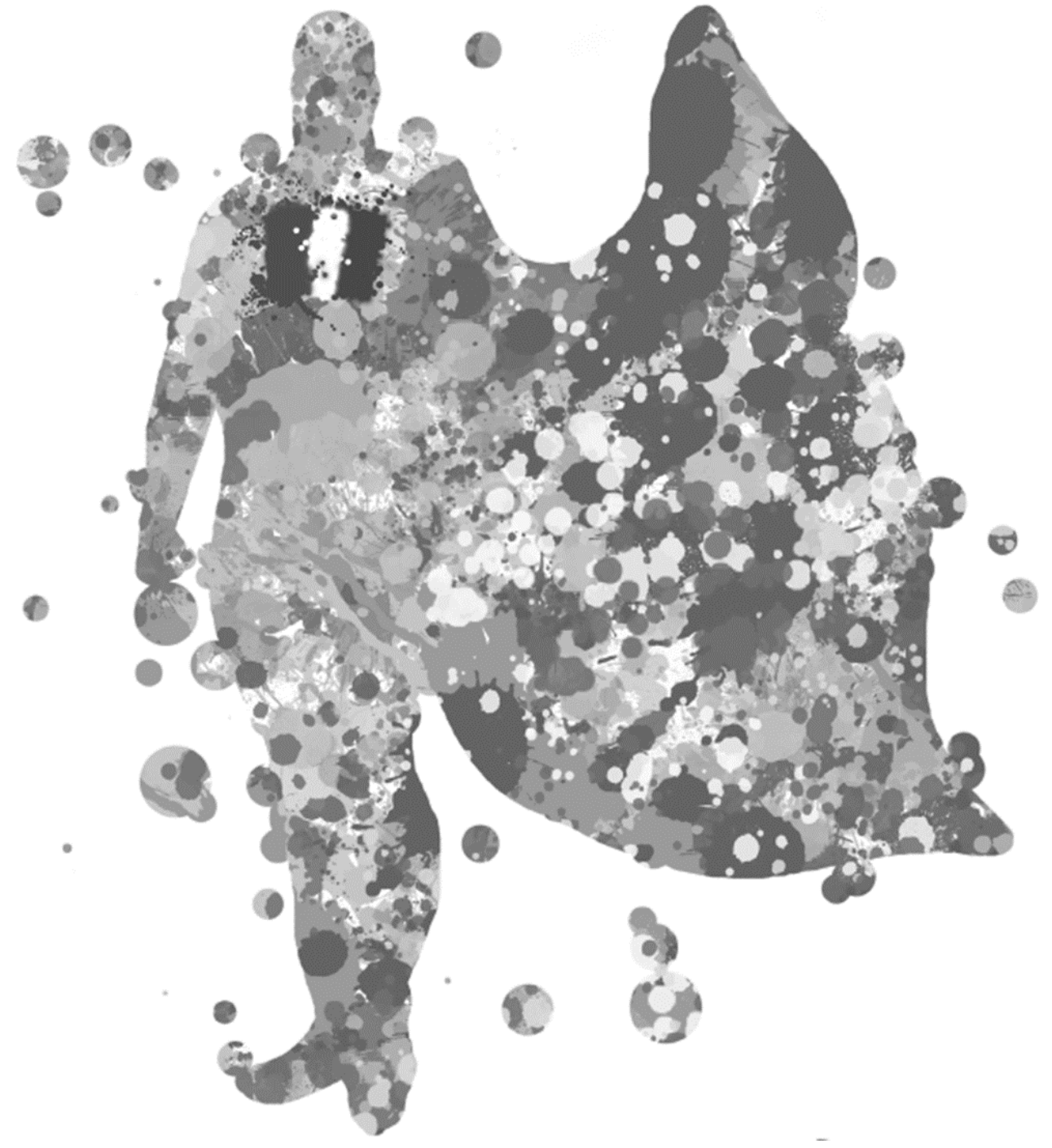


Summary

if
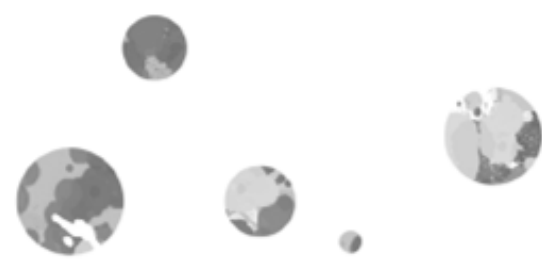

6

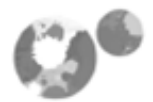


Cardiovascular diseases (CVD) are the leading cause of death worldwide, representing $31 \%$ of all global deaths. CVD are defined by several diseases of the heart and blood vessels, and involve several disorders, including coronary heart disease (CHD), peripheral arterial disease and cerebrovascular disease. One of the underlying causes of CHD is atherosclerosis. Dyslipidemia, characterized by a high level of low-density lipoprotein cholesterol (LDL-C) and triacylglycerol (TAG), and a low level of high-density lipoprotein cholesterol (HDL-C), is an important risk factor for the development of atherosclerosis. Although an inverse relationship between serum HDL-C concentrations and CVD risk has been found in several epidemiological studies, raising HDL-C levels failed to reduce CVD risk. It becomes, however, more and more accepted that improving HDL functionality represents a better target to reduce CVD risk. Indeed, a strong inverse relationship between HDL-mediated cholesterol efflux capacity, and the prevalence and the incidence of coronary artery disease (CAD) has been demonstrated in several epidemiological studies, and HDL-mediated cholesterol efflux was a strong predictor of CAD.

The research presented in this thesis focused on (1) a systematic review discussing factors and ABC-transporters that are associated with HDL-mediated cholesterol efflux in humans, and on (2) modulators of ABCA1-mediated cholesterol efflux from macrophages in humans. For this, the associations between genetic determinants, molecular factors, such as microRNAs (miRNAs), and environmental factors, i.e. diet and weight loss, and cholesterol efflux capacity were investigated.

In chapter 2, associations between ABC-transporters, HDL particle characteristics, subjects' characteristics, HDL-C raising drugs, lifestyle, genetic background, as well as acute and low-grade systemic inflammation and HDL-mediated cholesterol efflux were reviewed. These studies showed that in particular small pre $\beta-1$ HDL is an important determinant of HDL-mediated cholesterol efflux. This seems to contradict results from epidemiological studies, suggesting that large $\mathrm{HDL}_{2}$ particles are related to a reduced CVD risk. Chapter 3 discusses the association between single nucleotide polymorphisms (SNPs) and HDL-mediated cholesterol efflux in healthy volunteers. In this study, SNPs coding for key genes involved in the reverse cholesterol transport pathway from 50 men and 57 women were determined, and HDL-mediated cholesterol efflux was measured by incubating radiolabeled- ${ }^{3} \mathrm{H}-$ cholesterol loaded murine J774 macrophages overexpressing ABCA1 with apolipoprotein B-depleted serum. HDL-mediated cholesterol efflux was related to SNPs located in ABCA1, ABCG1, LXR $\beta$ and CETP. However, there is no evidence in the literature so far that these SNPs are related with a change in the risk to develop CVD. Further, ABCA1-mediated cholesterol efflux was positively associated with the use of statins and HDL-C concentrations, and a negative trend was observed for 
age, while no associations were found for gender and BMI. In chapter 4, the effect of theobromine consumption on fasting and postprandial cholesterol efflux was studied. In addition, three miRNAs known to be associated with HDL particles were selected, and their association with HDL-efflux capacity was investigated. In this randomized double-blind cross over study 30 overweight and 14 obese healthy men and women were included. Subjects were asked to consume $500 \mathrm{mg}$ of theobromine or placebo daily for 4 weeks. At the end of the intervention, fasting and postprandial samples were collected. Cholesterol efflux was measured in BODIPY-labeled murine J774 macrophages overexpressing the ABCA1 transporter, and miRNAs levels (miR92a; miR-223 and miR-135a*) were quantified. This study showed that high-fat meal intake increased postprandial cholesterol efflux capacity, as well as miR-92a and miR-223 levels. However, 4 weeks of theobromine consumption did not increase HDL-mediated cholesterol efflux capacity at baseline and postprandially. In addition, theobromine could exert anti-atherogenic properties by reducing miR-92a levels. Chapter 5 describes the effect of plant sterol and plant stanol consumption on cholesterol efflux and on CETP (cholesteryl ester transfer protein) mass and activity in 107 healthy men and women. This chapter used results from two studies previously performed at Maastricht University. In the first study subjects had to consume a control margarine or a margarine enriched with plant stanol esters for 8 weeks. In the second study, subjects were asked to consume a control margarine, or a plant sterol ester enriched margarine, or a plant stanol ester enriched margarine for 85 weeks. Cholesterol efflux was measured by incubating radiolabeled- ${ }^{3} \mathrm{H}-$ cholesterol loaded murine J774 macrophages overexpressing ABCA1 with apolipoprotein B-depleted serum. Plant stanol intake significantly decreased CETP mass, but had no effect on CETP activity and cholesterol efflux. No effects of plant sterols were found on any of these parameters. Finally, chapter 6 describes the effect of diet-induced weight loss on HDL-mediated cholesterol efflux and CETP activity in abdominally obese men. Cross-sectional differences at baseline between normalweight and abdominally obese men were also investigated. Twenty-five apparently healthy, normal-weight men and 52 abdominally obese men were included in this study. Abdominally obese subjects were randomly allocated to a dietary weight-loss intervention group or a no-weight loss control group. Individuals from the intervention group followed a very-low-calorie diet for 6 weeks to obtain a waist circumference below $102 \mathrm{~cm}$, followed by a weight-stable period of 2 weeks. Cholesterol efflux was measured in BODIPY-labeled murine J774 macrophages. CETP activity was measured by quantifying the transfer of CE from radiolabeled exogenous HDL to apoB-containing lipoproteins. Cross-sectionally, cholesterol efflux was negatively associated with the body mass index, while a positive association was found for CETP activity. However, diet-induced weight loss of 10 $\mathrm{kg}$ did not improve these two parameters. It would be of interest to address in future 
studies the importance of a longer period of stable body weight and the involvement of other transporters on efflux pathways.

Taken together, the research studies described in this dissertation were designed to assess how HDL functionality, i.e. HDL cholesterol efflux capacity, can be modulated and improved in humans. It was demonstrated that SNPs of genes involved in HDL metabolism are related to the ability of HDL to promote cholesterol efflux. Unfortunately, the dietary factors investigated as well as weight loss were not associated with an improvement in HDL-mediated cholesterol efflux. Further studies are therefore needed in order to determine the best dietary strategies to improve cholesterol efflux capacity, and to ultimately reduce CVD risk. 


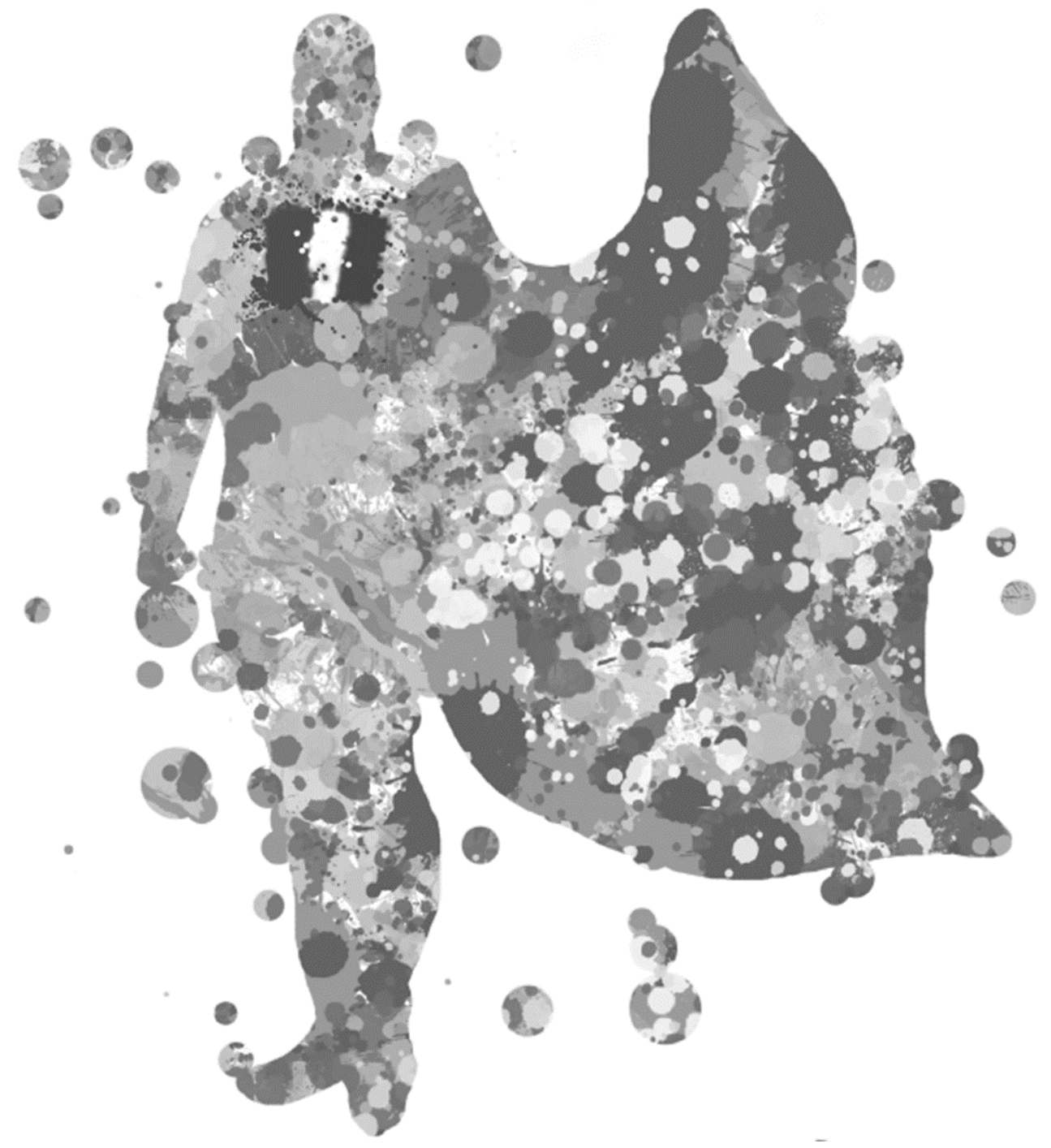




\section{Résumé}
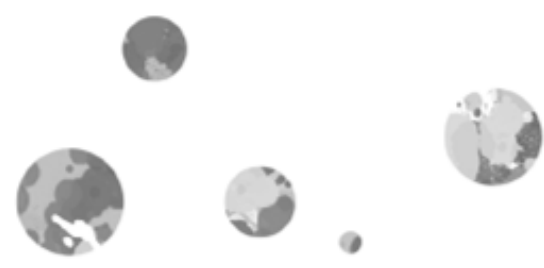

6

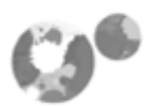


Les maladies cardiovasculaires (MCV) sont la principale cause de décès dans le monde, représentant $31 \%$ de la mortalité mondiale totale. Les MCV sont définies par plusieurs maladies du cœur et des vaisseaux sanguins et implique un ensemble de troubles comprenant les maladies coronariennes, les artériopathies périphériques et les maladies cérébro-vasculaires. Une des causes sous-jacentes des maladies coronariennes est l'athérosclérose. La dyslipidémie, caractérisée par un taux élevé du cholestérol des lipoprotéines de basse densité (LDL-C) et des triglycérides (TG), et un faible taux du cholestérol des lipoprotéines de haute densité (HDL-C), est un facteur de risque important dans le développement de l'athérosclérose. Bien qu'une association inverse entre les concentrations sériques de HDL-C et le risque de MCV ait été constatée dans plusieurs études épidémiologiques, l'élévation des concentrations de HDL-C n'a pas réussi à réduire le risque de $\mathrm{MCV}$. Il est de plus en plus accepté que stimuler la fonctionnalité des particules HDL constitue une meilleure cible pour réduire le risque de $\mathrm{MCV}$. En effet, une forte relation inverse entre l'efflux de cholestérol des macrophages vers les HDL et la prévalence et l'incidence des MCV a été démontrée, et l'efflux de cholestérol est un prédicteur des $\mathrm{MCV}$.

Les travaux de recherche présentés dans cette thèse ont porté sur (1) une analyse systématique des facteurs et des transporteurs $\mathrm{ABC}$ associés à l'efflux de cholestérol médiés par les particules HDL chez l'humain et (2) des modulateurs de l'efflux de cholestérol des macrophages vers les HDL chez l'humain, via le transporteur ABCA1. Pour ce faire, les associations entre les déterminants génétiques, les facteurs moléculaires, tels que les micro-ARN (miARN), et les facteurs environnementaux, tels que l'alimentation et la perte de poids, et l'efflux de cholestérol ont été étudiés.

Dans le chapitre 2, les associations entre les transporteurs $\mathrm{ABC}$, les caractéristiques des particules HDL, les caractéristiques des sujets, les médicaments destinés à augmenter le taux de HDL-C, le mode de vie, les antécédents génétiques, ainsi que l'inflammation systémique de bas grade, et l'efflux de cholestérol médié par les particules HDL ont été étudiés. Ces études ont montré que les petites particules pre $\beta$ - 1 HDL sont d'importants déterminants dans l'efflux de cholestérol. Cela semble contredire les résultats d'études épidémiologiques qui suggèrent que les grandes particules de HDL2 sont liées à un risque réduit de MCV. Le chapitre 3 traite de l'association entre les polymorphismes nucléotidiques (SNP) et l'efflux de cholestérol médié par les particules HDL chez des volontaires sains. Dans cette étude, des SNPs codant pour des gènes clés impliqués dans la voie de transport du cholestérol inverse chez 50 hommes et 57 femmes ont été déterminés, et l'efflux de cholestérol a été mesuré en incubant des macrophages murins J774 marqués radioactivement à l'aide de $3 \mathrm{H}$-cholestérol surexprimant le transporteur ABCA1 
avec du sérum dépourvu d'apolipoprotéines-B (apoB). Nous avons conclu que l'efflux de cholestérol médié par ABCA1 était positivement associé à l'utilisation des statines et aux concentrations de HDL-C, et une tendance négative a été observée pour l'âge. De plus, la capacité d'efflux de cholestérol était liée aux SNPs codant pour les gènes ABCA1, ABCG1, LXR $\beta$ et CETP. Cependant, il n'y a jusqu'à présent aucune preuve dans la littérature que ces SNP sont liés à un changement dans le risque de développer des MCV. De plus, l'efflux de cholestérol médié par ABCA1 était positivement associé avec l'utilisation de statines et les concentrations de HDL-C, et une tendance négative a été observée pour l'âge, alors qu'aucune association n'a été trouvée pour le sexe et l'indice de masse corporelle (IMC). Dans le chapitre 4, nous avons étudié l'effet de la consommation de théobromine sur l'efflux de cholestérol à jeun et postprandial. De plus, trois miARNs connu pour être associés aux particules de HDL ont été sélectionnés, et leur association avec la capacité d'efflux de HDL a été étudiée. Dans cette étude randomisée en cross-over et double aveugle, 30 hommes et femmes en surpoids et 14 hommes et femmes obèses en bonne santé ont été inclus. Les sujets ont dû consommer $500 \mathrm{mg}$ de théobromine ou de placebo par jour pendant 4 semaines. À la fin de l'intervention, des échantillons à jeun et des échantillons postprandiaux ont été prélevés. L'efflux de cholestérol a été mesuré dans des macrophages murins J774 marqués à l'aide de BODIPY-cholestérol surexprimant le transporteur ABCA1. Les concentrations de chaque miARNs (miR92a, miR-223 et miR-135a*) ont été quantifiés. Cette étude a montré que la consommation d'un repas riches en graisses augmente la capacité d'efflux de cholestérol, ainsi que les concentrations de miR-92a et miR-223. Cependant, 4 semaines de consommation de théobromine n'ont pas amélioré la capacité d'efflux de cholestérol médiée par le HDL, à jeun et à la suite du repas. De plus, la théobromine pourrait exercer des propriétés anti-athérogènes en réduisant les concentrations de miR-92a. Le chapitre 5 décrit l'effet de la consommation de stérols et de stanols végétaux sur l'efflux de cholestérol ainsi que sur l'activité et la masse de CETP (cholesteryl ester transfer protein), chez 107 hommes et femmes en bonne santé. Ce chapitre regroupe les résultats de deux études réalisées précédemment à l'Université de Maastricht. Dans la première étude, les sujets devaient consommer une margarine témoin ou une margarine enrichie en stanols végétaux pendant 8 semaines. Dans la deuxième étude, il a été demandé aux sujets de consommer une margarine témoin, une margarine enrichie en stérols végétaux ou une margarine enrichie en stanols végétaux pendant 85 semaines. L'efflux de cholestérol a été mesuré dans des macrophages murins $\mathrm{J} 774$ marqués à l'aide de $3 \mathrm{H}$-cholestérol surexprimant le transporteur ABCA1. La consommation de stanols végétaux a significativement diminué la masse de CETP, mais n'a eu aucun effet sur l'activité de la CETP et l'efflux de cholestérol. De plus, aucun effet des stérols végétaux n'a été trouvé sur aucun des paramètres testés. Enfin, le chapitre 6 décrit l'effet de la perte 
de poids, induite par un régime alimentaire, sur l'efflux de cholestérol et l'activité de la protéine impliquée dans le transfert des esters de cholestérol entre les différentes lipoprotéines, i.e. CETP, chez des hommes présentant une obésité abdominale. De plus, les différences entre les hommes de poids normaux et les hommes obèses ont également été étudiées. Vingt-cinq hommes apparemment en bonne santé, de poids normal et 52 hommes obèses ont été inclus dans cette étude. Les sujets présentant une obésité abdominale ont été répartis de façon aléatoire dans le groupe d'intervention ou le groupe témoin sans perte de poids. Les individus du groupe d'intervention ont suivi un régime très faible en calories pendant 6 semaines pour obtenir un tour de taille inférieur à $102 \mathrm{~cm}$, suivi d'une période de 2 semaines au cours de laquelle le poids devait rester stable. L'efflux de cholestérol a été mesuré dans des macrophages murins J774 marqués à l'aide de BODIPY-cholestérol, et surexprimant le transporteur ABCA1. L'activité de CETP a été mesurée en quantifiant le transfert de cholestérol estérifiés entre des particules exogène de HDL vers les lipoprotéines riches en apoB. Il a été démontré que l'efflux de cholestérol était négativement associé à l'IMC, tandis qu'une association positive a été observée pour l'activité de CETP. Toutefois, la perte de poids de $10 \mathrm{~kg}$ induite par le régime alimentaire n'a pas amélioré ces deux paramètres. Il serait intéressant d'aborder, dans des études futures, l'importance d'une plus longue période de stabilité du poids corporel et l'implication de d'autres transporteurs dans l'efflux de cholestérol.

Les études de recherche décrites dans cette thèse ont été conçues pour évaluer comment la fonctionnalité des particules de HDL peut être modulée et améliorée chez l'humain. Il a tout d'abord été démontré que des SNP codant pour des gènes impliqués dans le métabolisme des HDL sont liés à la capacité des particules de HDL à stimuler l'efflux de cholestérol. Malheureusement, les facteurs alimentaires étudiés ainsi que la perte de poids n'ont pas été associés à une amélioration de l'efflux de cholestérol médié par le HDL. D'autres études sont donc nécessaires afin de déterminer les meilleures cibles pour améliorer la capacité d'efflux de cholestérol et, à terme, réduire le risque de $\mathrm{MCV}$. 


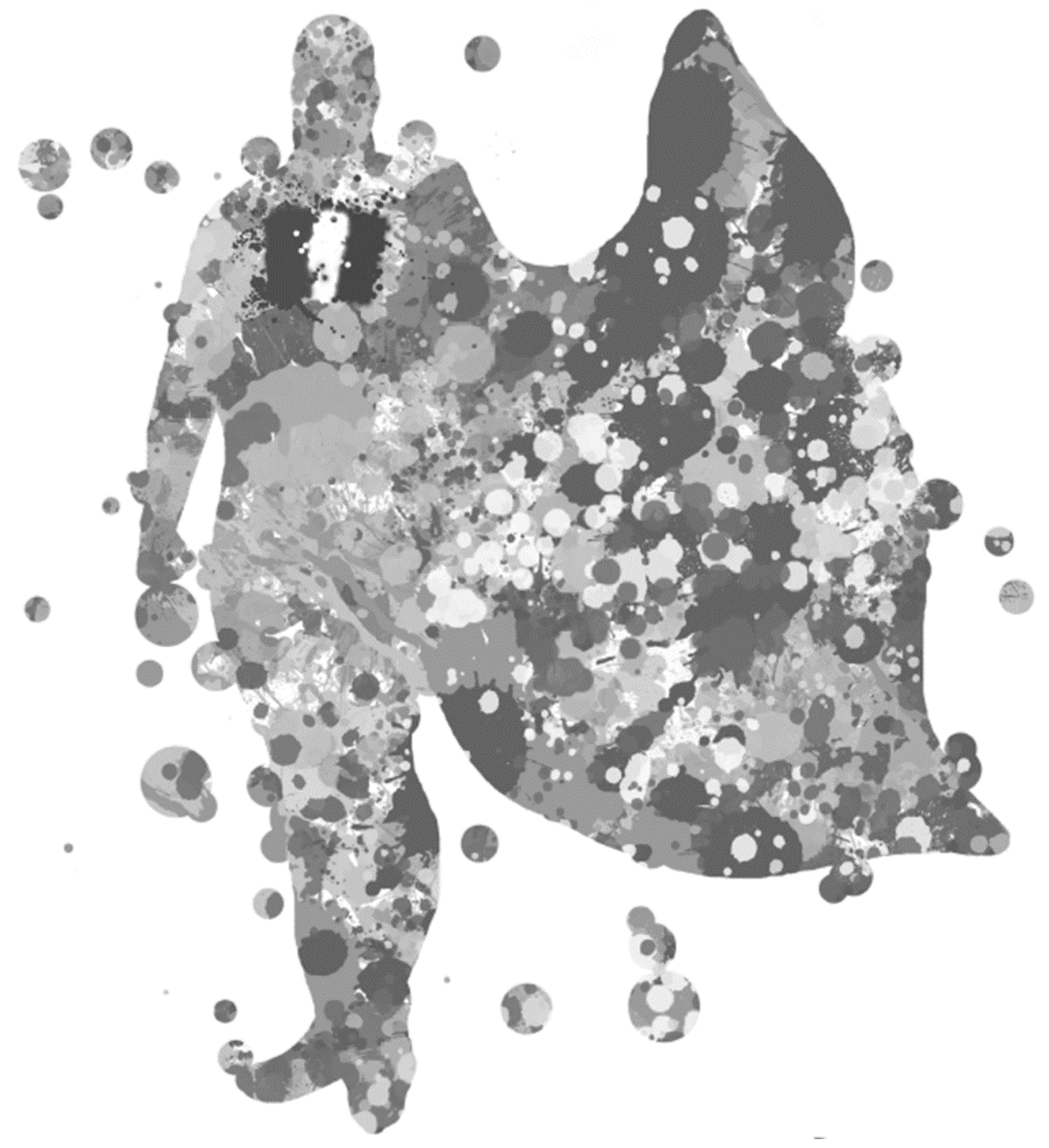




\section{Valorisation}

is

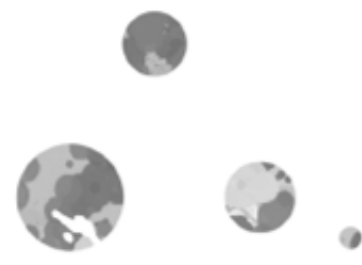




\section{Social and economic relevance}

Despite considerable progress in tackling the development of cardiovascular disease (CVD), it remains one of the leading causes of death. On a yearly basis, CVD is responsible for 3.9 million of death in Europe and account for $31 \%$ of all global death worldwide. In 2015, more than 85 million people were living with CVD in Europe. In this context, CVD represents also a major economic burden. Overall, in Europe, it is estimated that CVD cost 210 billion euros yearly, including cost due to health care, loss of productivity and to informal care of people with CVD [1,2]. Therefore, it is essential to better understand the underlying mechanism leading to CVD, in order to develop effective strategies to prevent or delay CVD development.

Atherosclerosis is the main underlying pathology in CVD development and dyslipidemia is an important risk factor for atherosclerosis. Interventions targeting low-density lipoprotein cholesterol (LDL-C) have been successfully developed [3, 4]. However, subjects using therapies to reduce their LDL-C concentrations often fail to reach the desired LDL-C concentrations, leading to a remaining risk for CVD. It is therefore essential to discover other approaches to further reduce CVD risk. Increasing high-density lipoprotein cholesterol (HDL-C) concentrations is also an attractive target, as many epidemiological studies have found an inverse association between HDL-C concentrations and CVD risk $[5,6]$. However, raising HDL-C levels failed to reduce CVD risk [7], while recent evidences suggest that increasing the cholesterol efflux capacity of HDL particles protects against the development and the recurrence of CVD [8-10]. It is therefore time to switch the research focus from HDL-C to HDL functionality, i.e. HDL-mediated cholesterol efflux capacity.

The most important behavioral risk factors for CVD are unhealthy diets, lack of physical activity, smoking and an excessive use of alcohol [2]. Foods containing bioactive components, so-called functional foods, can be used for the prevention of CVD, and beneficial effects on lipid metabolism have already been shown [11]. However, functional foods aiming at increasing HDL cholesterol efflux capacity are not yet available, and development of such food items is therefore needed. In this thesis, we aimed to identify potential ingredients that might improve HDLmediated cholesterol efflux. We first hypothesized that theobromine could be one of the potentially healthy components in cocoa. Indeed, dark chocolate consumption is a highly consumed food and has been associated with a reduction in the risk to develop cardiovascular disease (CVD) [12]. It is therefore of interest to identify which compound(s) in cocoa is/are responsible for the beneficial effects, as the identified compound(s) could subsequently be used as functional ingredients. 
Unfortunately, we did not find that theobromine had any beneficial effects on HDL cholesterol efflux capacity. Secondly, nowadays many functional food products enriched in plant sterol and plant stanol, such as margarines, have been developed and were shown to reduce cholesterol concentrations [13]. However, in line with theobromine, plant sterol and stanol enriched products did also not improve HDL cholesterol efflux capacity.

\section{Relevance of measurements}

The research described in this dissertation helps to better understand how HDL cholesterol efflux capacity can be modulated and improved in humans, in order to reduce CVD risk. In 2016, over 1.9 billion of the global adult population was overweight; of these more than 650 million were obese. Because people with overweight and obesity are at high risk to develop CVD [14], the effect of weight loss on HDL efflux capacity is therefore of clinical importance when studying CVD risk. In addition, the majority of the population spends a large part of the day in the postprandial state, and disturbances in postprandial lipoproteins metabolism are important risk markers for CVD [15, 16]. In this context, measuring not only fasting but also postprandial cholesterol efflux capacity is of importance. Furthermore, the microRNAs (miRNAs) signature has been demonstrated to be altered in diseased subjects [17]. A better understanding of the involvement of specific miRNAs in the modulation of HDL-mediated cholesterol efflux is of interest, and could help for the development of strategies using miRNA-based therapies. MiRNAs therapies are currently being developed, and Miravirsen, a miR-122 antagonist, is currently in phase II clinical trials and may be the first miRNA-targeted drug receiving FDA (Food and Drug Administration) approval for the treatment of hepatitis C [18, 19]. Ultimately, the discovery of new therapies targeting the increase of HDL functionality, either via miRNAs or not, would help to reduce the costs generated by the treatment of CVD.

\section{Translation into practice}

With the present thesis, we expect to increase the awareness of the medical, societal and economic consequences of CVD, and to highlight the potential impact of genetic background, miRNAs and nutrition on HDL functionality as related to CVD risk. The results presented in this thesis have been presented at several national and international conferences. Furthermore, the research findings have been or will be submitted to international peer-reviewed scientific journals. 


\section{References}

1. Network, E.H. European Cardiovascular Disease Statistics 2017. 2017; Available from: http://www.ehnheart.org/cvd-statistics.html.

2. Organization, W.H. Cardiovascular diseases (CVDs) fact sheet No. 317. May 2017; Available from: http://www.who.int/mediacentre/factsheets/fs317/en/.

3. Stein, E.A., Low-density lipoprotein cholesterol reduction and prevention of cardiovascular disease. Mayo Clin Proc, 2009. 84(4): p. 307-9.

4. Stroes, E., Statins and LDL-cholesterol lowering: an overview. Curr Med Res Opin, 2005. 21 Suppl 6: p. S9-16.

5. Barter, P., et al., High density lipoproteins (HDLs) and atherosclerosis; the unanswered questions. Atherosclerosis, 2003. 168(2): p. 195-211.

6. Choi, B.G., et al., The role of high-density lipoprotein cholesterol in the prevention and possible treatment of cardiovascular diseases. Curr Mol Med, 2006. 6(5): p. 571-87.

7. Rader, D.J. and G.K. Hovingh, HDL and cardiovascular disease. Lancet, 2014. 384(9943): p. 618-25.

8. Khera, A.V., et al., Cholesterol efflux capacity, high-density lipoprotein function, and atherosclerosis. N Engl J Med, 2011. 364(2): p. 127-35.

9. Rohatgi, A., et al., HDL cholesterol efflux capacity and incident cardiovascular events. N Engl J Med, 2014. 371(25): p. 2383-93.

10. Saleheen, D., et al., Association of HDL cholesterol efflux capacity with incident coronary heart disease events: a prospective case-control study. Lancet Diabetes Endocrinol, 2015. 3(7): p. 507-13.

11. Sikand, G., P. Kris-Etherton, and N.M. Boulos, Impact of functional foods on prevention of cardiovascular disease and diabetes. Curr Cardiol Rep, 2015. 17(6): p. 39.

12. Buitrago-Lopez, A., et al., Chocolate consumption and cardiometabolic disorders: systematic review and meta-analysis. Bmj, 2011. 343: p. d4488.

13. Kamal-Eldin, A. and A. Moazzami, Plant sterols and stanols as cholesterol-lowering ingredients in functional foods. Recent Pat Food Nutr Agric, 2009. 1(1): p. 1-14.

14. Organization, W.H. Obesity and overweight fact sheet No. 311 October 2017. 2017; Available from: http://www.who.int/mediacentre/factsheets/fs311/en/.

15. Karpe, F., Postprandial lipoprotein metabolism and atherosclerosis. J Intern Med, 1999. 246(4): p. 341-55.

16. Cohn, J.S., Are we ready for a prospective study to investigate the role of chylomicrons in cardiovascular disease? Atheroscler Suppl, 2008. 9(2): p. 15-8.

17. Vickers, K.C., et al., MicroRNAs are transported in plasma and delivered to recipient cells by high-density lipoproteins. Nat Cell Biol, 2011. 13(4): p. 423-33.

18. van der Ree, M.H., et al., Long-term safety and efficacy of microRNA-targeted therapy in chronic hepatitis C patients. Antiviral Res, 2014. 111: p. 53-9.

19. Drury, R.E., D. O'Connor, and A.J. Pollard, The Clinical Application of MicroRNAs in Infectious Disease. Front Immunol, 2017. 8: p. 1182. 


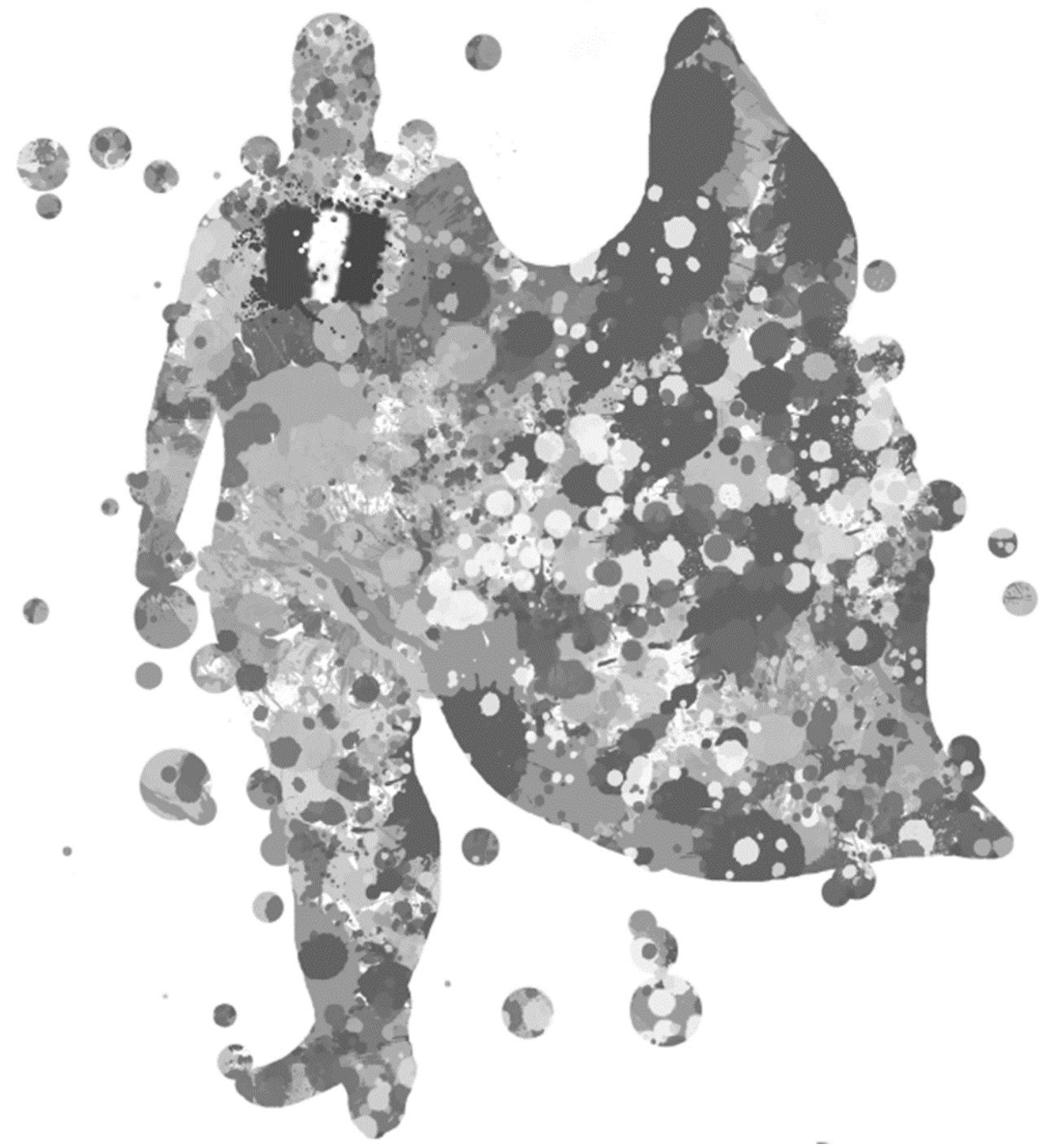


Acknowledgements

is

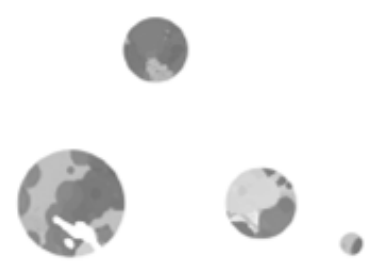

6 
This is it! My book is finally finished, and it is now time to thank all the people that supported me and made this possible.

First of all, I would like to thank my supervisors, Ronald and Jogchum. Thank you for giving me the opportunity to come to Maastricht and do my $\mathrm{PhD}$. I have learnt a lot from you both.

Ronald, thank you for all your constructive comments on my articles. I did get frustrated sometimes when opening your corrections but, in the end, I know they made my articles way better. But for all the other PhDs, remember: version 23 is the maximum! Thank you for being so patient with me while trying to explain me statistics, or teach me Dutch. But I do know some very important Dutch words now thanks to you: waterglijbaan, vloerbedekking, smeerpoets and jokkebrok. Also, sorry for "killing" your mouse. I know I can be quite stubborn but I will finally admit it: you are always right! $:-$

Jogchum, I had really good times going to congresses with you (even in Maikammer $\left.{ }^{\wedge \wedge}\right)$. I remember that you were always still full of energy after a full day of presentations at the EAS and wanted to go for drinks. Thank you for your enthusiasm and for being always so optimistic.

To the members of my assessment committee, prof. dr. E.C.M. Mariman, prof. dr. E.A.L. Biessen, dr. C. van der Kallen, prof. dr. B. Staels and dr. P.L Zock, thank you for taking time to review my dissertation.

My lieve paranymphs, Marlies and Cyril, what would I have done without you both?!

Marliesje, I have so many things to thank you for. Thank you for being such a good friend and for your support. I really enjoyed our girls' night, our talks about boys, our movies/series nights, our crazy singing in the car... Thank you for always cooking for me and making me eat my vegetables. Thank you for always pushing me on my bike, Maastricht is such a hill! And for helping me to survive the camping at Lowlands (but never again ${ }^{\wedge} \wedge$ ). I'm looking forward to be your cat (and wife) $)($.)

Cyril, you helped me so much when I first arrived in Maastricht. Thank you for having been here for me. We have so much in common, especially our unconditional love for the serie Friends (and mostly Ross: "what's in the cup Ross?"). I remember when I first met you on the Nutrim day, it felt so natural and we became friends right away. I wish you all the best with Pascalle and for your next adventure together 
with your soon-to-be-born baby (which I secretly hope will be named LaurentVoulzy...^^).

Marlies, Cyril, Roel and Dirk, I loved watching Game of Thrones (tin tin tintintin tin tintintin tin tintintin: I think our singing is getting better and better) and Star Wars with you guys. No matter the distance, we always managed to see each other for these special occasions.

To all the nerds, Alex (mon compatriote français), Dennis (des bois), Dirk (such a derk), Emmani (my poffertje), Jan (the German beast), Marlies (the vengeful spirit of the night), Max (the absolute Exploding Kittens' fan), Niels (you are such an adult) and Yvo (shubae), you guys are the best! Thank you for all the awesome times, lunches, dinners, board games, meme wars, weird videos ("you gonna get wet") and songs ("why you always lying"). You all made my PhD way more enjoyable. Cheers, to violence!!

Emmani, I always loved when you were talking French with me. I could not have wished for a better person to do the practical RNA/DNA with. By the way, can I touch your planets? And please, if you have some Rituals' samples you don't want, think of me (and give them to me) $\odot$.).

Alex, it was super nice to have another Frenchie in the group, who shared love for fromages and who had the same type of lunch as me (although a little bit healthier). Best of the luck for the end of your PhD.

Yvo, thank you for all good times spend together while shopping, having a nice (fatty) dinner, playing PlayStation and watching movies.

To all my PhuN (fun :) colleagues, Cara, Dorien, Ellen, Elske, Eva, Herman, Jordi, José, Kylie, Lea, Mathijs, Maud, Maurice, Merel, Peter, Sabine, Tanja and Virginie, as well as the previous ones, Bibi, Daisy, Dorien, Lotte, Rezy and Sophie (Soso!), thank you for the "gezellig" times, the very fun group outings' and coffee breaks.

Eva, I will forever be jealous of your gorgeous hair! And I hope we can have many more girls' night together, watching stupid romantic comedies.

Sabine, I loved sharing the room with you for these few months. I really enjoyed our talks, mostly about Game of Thrones, Netflix and Thor. Thank you for your kindness, for your help with all the administrative paperwork, and for having been here for me when I was feeling down. 
Peter (Pierre pour les amis), I had so much fun with you!! Thank you for your help with the statistics, you are definitely a PP (Perfect Peter).

Maurice, thank you for your patience with all my questions and for your help in the lab.

Lotte, I had really the best time sharing the room with you for 3 years, and really enjoyed all of our conversations (definitely not about sciences $\odot$ ).

Guy, thank you for your support, and for lending me your amazing computer screen!! I had great times with you; especially when drinking red wine while listening old music records, and during your photography lessons.

Thanks to all the other people from the Human Biology and Movement Sciences department $(\mathrm{A}+\mathrm{B}$ !) for the always fun $\mathrm{HB} / \mathrm{BW}$ weekends and Friday borrels.

To Jos, you will be forever missed.

To my French compatriots:

Florian C., I'm gonna miss our lunch breaks together so much!! It was great to talk about geeky stuffs (TV shows, movies, board games,...), and be able to complain about... you know what ${ }^{\wedge} \wedge$

Florian B., what a small world! We definitely have to thank Gatien for introducing us! It was great to have another Frenchie to hang out with at work and outside. Thank you for cheering me up so many times.

And to Max and Fred, \#plaisir!

Sarah, it was great meeting you at the VLAG week. I'm glad that we always found a bit of time to meet up together.

Un grand merci à mes amis en France, et particulièrement à Emma, Fanny, Gégé, Inès, Max, Popo et Soso, pour être venu me voir à Maastricht.

Et bien évidemment, les meilleurs pour la fin. Papa, Maman et Julie, merci pour votre soutien tout au long de ma thèse et pour être toujours présents pour moi malgré la distance! Merci d'être venu me voir aux Pays Bas et de m'avoir ramené et cuisiné la nourriture française que j'aime tant. Revenir à la maison est toujours une bouffée d'oxygène $<3$ 


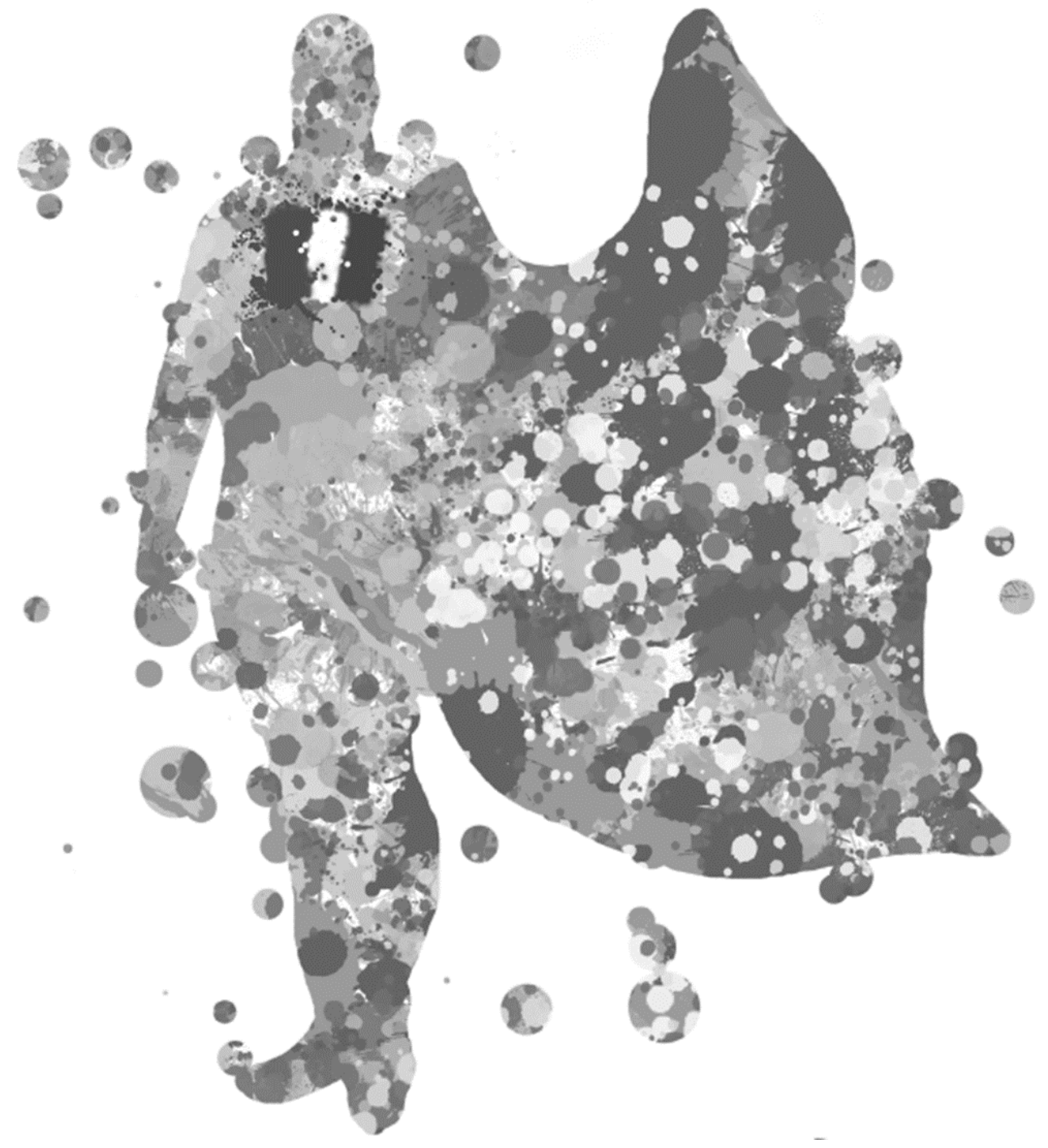




\section{Curriculum Vitae}

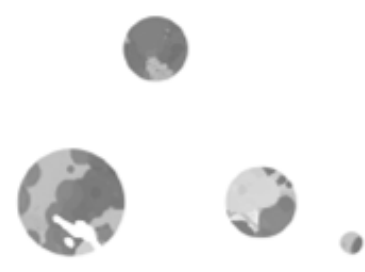

$6:$

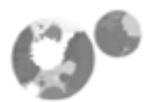


Charlotte was born in Parthenay, France, on the 19th of September 1989. She completed secondary school in 2007 at the Lycée Maurice Genevoix in Bressuire, France.

From 2007 until 2010 she studied Health Sciences with the specialization Animal Physiology and Neurosciences at the University of Poitiers, France. Her bachelor internship was performed at the French National Institute for Health and Medical Research (INSERM U957) in Nantes, France.

In 2012, she graduated with honors for the Research Master in Food Science and Human Nutrition at the University of Nantes, France. Her master internship was spent at the Nantes Atlantic College of Veterinary Medicine Food Science and Engineering, in the unit of Nutrition and Endocrinology. Under the supervision of dr. Veronique Leray, she investigated the effects of supplementation with omega-3 polyunsaturated fatty acids with or without curcumin on oxidative stress in obese and insulin-resistant dogs.

In December 2013, she was appointed as a PhD-student at the department of Nutrition and Movement Sciences at Maastricht University, the Netherlands, under the supervision of prof. dr. ir. Ronald P. Mensink and prof. dr. Jogchum Plat. During her $\mathrm{PhD}$ project, she investigated the impact of genetics, diet and weight loss on HDL-mediated cholesterol efflux. Charlotte also collaborated with dr. Fatiha Tabet from the University of New South Wales Sydney, Australia, in order to learn a new technique to isolate and purify HDL particles. These 4 years also gave her the opportunity to take part in various teaching activities. Charlotte has presented her project results at several national and international congresses. 


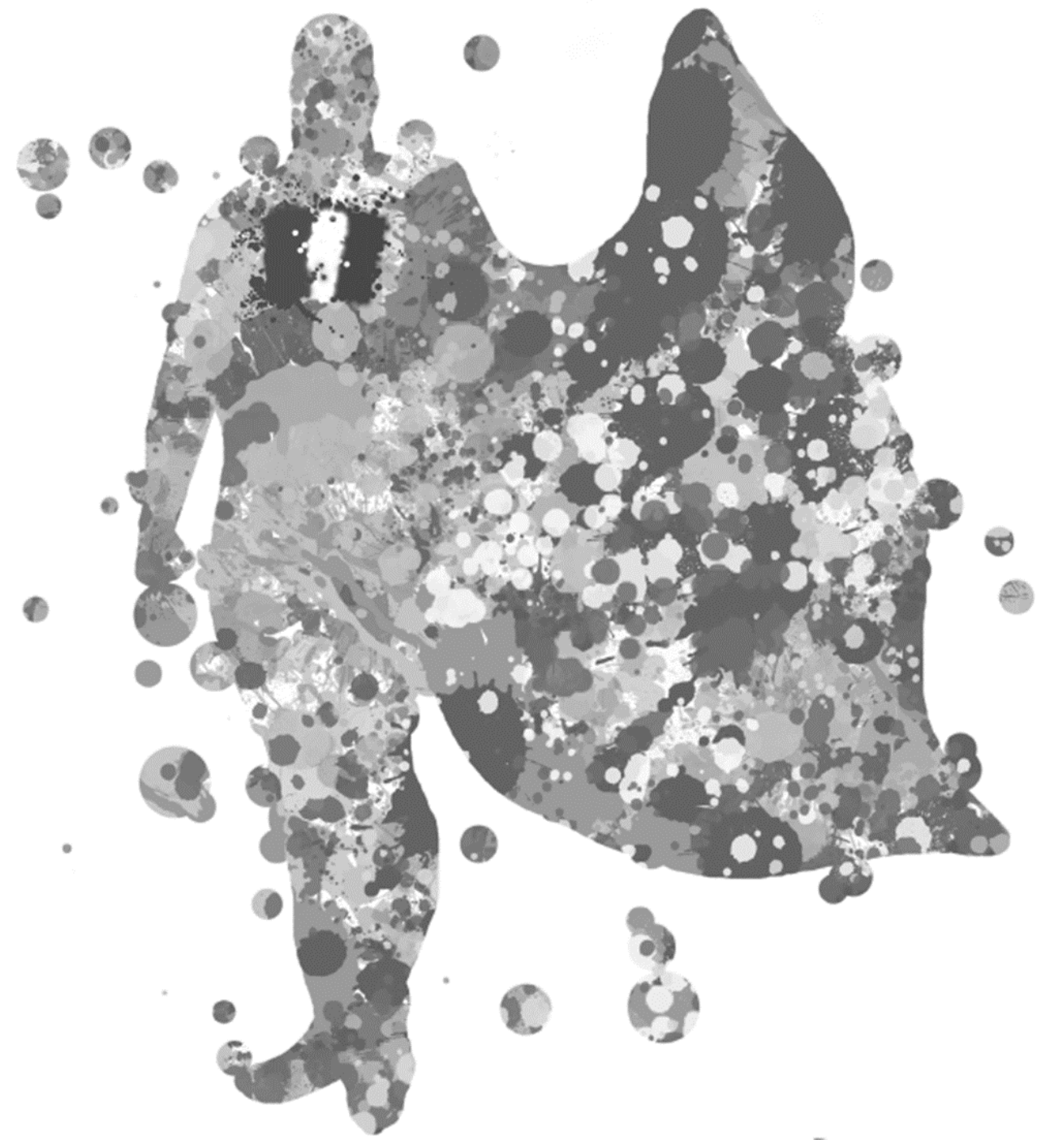




\section{List of publications}

is

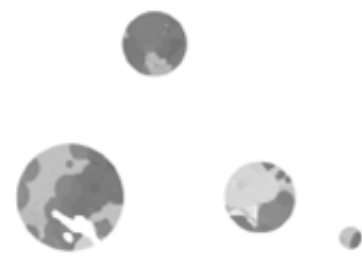

6

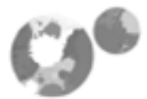




\section{Published}

Talbot CPJ, Plat J, Ritsch A, Mensink RP. Determinants of cholesterol efflux capacity in humans. Progress in Lipid Research. 2017; 69: 21-32

\section{Submitted}

Talbot CPJ, Plat J, Joris PJ, Konings M, Kusters YHAM, Schalkwijk CG, Ritsch A, Mensink RP. HDL cholesterol efflux capacity and CETP activity are associated with body mass, but are not changed by diet-induced weight loss: a randomized trial in abdominally obese men. Revision submitted

Talbot CPJ, Mensink RP, Smolders L, Bakeroot V, Plat J. Theobromine does not affect fasting and postprandial HDL cholesterol efflux capacity, while it decreases fasting miR-92a levels in humans. Submitted

\section{To be submitted}

Talbot CPJ, Mensink RP, Lutjohann D, Ritsch A, Plat J. Effects of plant sterol and stanol ester enriched margarine consumption on plasma cholesterol efflux capacity, CETP mass and CE transfer. To be submitted

Talbot CPJ, Plat J, Popeijus HE, Ritsch A, Mensink RP. SNPs located in ABCA1, ABCG1, LXR $\beta$ and CETP are associated with plasma cholesterol efflux. In preparation 